\title{
A SIMPLE MULTI-MODEL PREDICTION METHOD
}

\author{
Flavio A.M. Strutzel ${ }^{1}$, I. David L. Bogle ${ }^{2}$ \\ ${ }^{1}$ Petrobras Oil Company, President Bernardes Refinery (RPBC), Cubatão, São Paulo, \\ Brazil. \\ Tel.: +55 $113328-4421$. \\ E-mail address: flavio.ams@petrobras.com.br \\ ${ }^{2}$ Centre for Process Systems Engineering, Department of Chemical Engineering, \\ University College London, Torrington Place, London WC1E 7JE, UK \\ Tel.: +44 207679 3803; fax: +44 2073832348. \\ E-mail address: d.bogle@ucl.ac.uk (I. David L. Bogle)
}

\begin{abstract}
The present work introduces a new multi-model state-space formulation called simultaneous multi-linear prediction (SMLP), which is suitable for systems with significant gain variation due to nonlinearity.

Standard multi-model formulations usually make use of a partitioned state-space, i.e., a state-space that is divided into regions to shift parameters of the state update equation according to the current location of the state, with a view to having a better approximation of a nonlinear plant on each region. This multi-model framework, also known as linear hybrid systems framework, makes use of different boundaries or partition rules concepts, which vary from systems of linear inequalities, propositional logic rules, or a combination of these. This standard approach inevitably introduces discontinuities in the output prediction as the state update equation parameters shift noticeably.

Instead, the SMLP is built by defining and updating multiple states simultaneously, thus eliminating the need for partitioning the state-input space into regions and associating with each region a different state update equation. Each state's contribution to the overall output is obtained according to the relative distance between their identification (or linearisation) point and the current operating point, in addition to a set of parameters obtained through regression analysis. Unlike the methods belonging to the hybrid systems framework, no discontinuities are introduced in the output prediction
\end{abstract}


while using an SMLP system, as the weighting function is continuous and the transition between sub-models is smooth.

This method presents more accurate results than the use of single linear models while keeping much of their numerical advantages and their relative ease of development. Additionally, the SMLP draws data from step response models that can be provided by commercial, black box dynamic simulators, enabling it to be applied to large-scale systems.

In order to assess this methodology, an SMLP system is built for an activated sludge process (ASP) of a wastewater treatment plant, alongside a standard multi-model Piecewise Affine system generated by the same sub-models, and their output predictions are compared.

The controllability analysis and the case study presented in Strutzel and Bogle (2016) are extended and updated to this multi-model approach, yielding SMLP systems describing four alternative designs for a realistically sized crude oil atmospheric distillation plant.

Keywords

State-space models, Multi-model state-space, Multi-model MPC, Linear Hybrid Systems, Integrated Process Design and Control, Model Predictive Control (MPC), Zone Constrained Model Predictive Control, Controllability Analysis, Crude Oil Distillation, Activated Sludge.

\section{Introduction and motivation}

The motivation behind devising a system for the multi-model state-space representation of chemical processes was improving the accuracy of our controllability analysis methodology, presented in Strutzel and Bogle (2016). In that work, a standard linearised state-space model was used to predict plant behaviour, control performance and profitability. This reduced the numerical complexity of the integrated process design and methodology but led to compromises regarding its precision.

Hence, let us discuss this previous work briefly. Alternative process plants designs have different control performance. This will depend on aspects of the plant units and their configuration, creating both unit and system holdups and sensitivities, and on the 
type of control exercised (Morari, 1983; Edgar, 2004). Strutzel and Bogle (2016) presented a design approach using the Economic MPC Optimisation (EMOP) index as a tool for the assessment of different process designs based on a monetised measure of the control effort required in a number of scenarios.

The EMOP index has two main components: the sum of changes in revenue that occur due to moves in the manipulated variables (MVs), which can be described as a monetised measure of the control effort, and another term which penalises violations of the control zones (defined by the maximum and minimum values acceptable) of each controlled variable $(\mathrm{CV})$. Additional terms are included to ensure adequate transient behaviour as the system moves between the initial and final states. Using optimisation algorithms to find the minimum of the EMOP index equation yields an optimal design (Strutzel and Bogle, 2016). The EMOP index should not be mistaken for a standard closed-loop economic MPC problem. It is rather an open-loop reachability problem, which does not rely on any specific MPC algorithm or set of tuning parameters (details can be found in Section 5).

The assessment of control performance of large-scale systems uses data from commercial, black-box nonlinear dynamic simulation packages that are popular in the chemical industry. These state-of-the-art simulators are the tools of choice of most process design teams. We use the integrated design and control approach for large-scale, complex processes for which the full set of equations is unavailable.

Data from one such simulator was used in Strutzel and Bogle (2016) to identify linear state-space models that predicted the dynamic behaviour of each flowsheet, avoiding the task of solving nonconvex optimisation problems. This is a better option than using the nonlinear simulation package directly since that is very computationally intensive when using solvers to find the optimal state. However, using linear models to predict the behaviour of nonlinear plants is inevitably going to result in some error. This problem was addressed by defining the EMOP index as an interval (bounded by best and worst scenarios) within which the true controllability index must be contained.

In this paper, an alternative approach for reducing nonlinearity-introduced error is presented while keeping all the advantages of using linear models. This solution, a new multi-model state-space approach called simultaneous multi-linear prediction (SMLP), is described in Section 3. The main idea is to ensure that the evaluation of the index and the subsequent ranking of flowsheets are as accurate as possible within a reasonable period, 
thus rendering the EMOP index more resilient to process nonlinearity. While not the focus of this paper, the SMLP can also be used for process control purposes. Since its open loop stability can be guaranteed, as it will be shown, the use of SMLP is particularly interesting for MPC.

A major part of MPC's appeal in industry stems from the use of a linear finite step response model of the process and a quadratic objective function. When MPC is employed on processes with significant nonlinearity, the application of a linear modelbased controller may have to be limited to relatively small operating regions. Specifically, since the computations are entirely based on the model prediction, the accuracy of the model has a significant effect on the performance of the closed-loop system (Gopinath et al, 1995). Hence, the capabilities of MPC will degrade as the operating level moves away from its original design level of operation (Dougherty and Cooper, 2003). Enhancing prediction accuracy will increase closed-loop stability and help avoid overdamped or underdamped responses.

MPC with SMLP also has one advantage over MPC with nonlinear models (NMPC): while NMPC involves a non-convex optimisation problem for which local minima can found but nothing can be said about the global minimum (Kantner and Primbs, 1997), the use of an SMLP prediction provides a convex cost function for which the global minimum can be easily found without the need for relaxation.

This paper is organised into 7 Sections. In this first Section, the problem being addressed was introduced and the motivation for the novel SMLP method was presented. In the second Section, some background is offered into the existing multi-model system framework. A discussion is presented highlighting limitations concerning this framework and why an alternative scheme such as the SMLP can be advantageous. In the third Section, the design methodology is presented and the properties of SMLP systems such as stability and reachability are discussed. In the fourth Section, an SMLP system is built for an activated sludge process (ASP) of a wastewater treatment plant alongside an equivalent Piecewise Affine system, and their output predictions are compared to benchmark the SMLP. The fifth Section contains a short review of the EMOP index as well as a small update to allow variable product prices to be accounted for. In the sixth Section, a case study consisting of 4 alternative designs for a crude oil distillation process is provided. The plants were represented as SMLP systems and their dynamic properties 
were evaluated using the EMOP index. In the seventh and last Section, the conclusions concerning the SMLP method are presented.

\section{A brief review of the multi-model systems}

In this Section, some information is provided about the state-of-art multi-model approaches that are currently available alternatives as to the SMLP. Such approaches can be loosely classified into a linear hybrid systems framework, in which a hybrid system is a collection of digital programs interacting with each other and with an analogue environment. Each logic state of the digital part of the hybrid system acts on the analogue part inducing a different operational mode. On the other hand, the evolution of the analogue part triggers switches in the states of the digital part. Many physical phenomena admit a natural hybrid description, such as control valve saturation; digital controllers embedded in a continuous process, which act on on/off valves; process equipment switching; switching on and off the electrical motor of pumps and compressors, and many other complications that demand adequate modelling. Moreover, some of the linear hybrid classes can be used to reduce the prediction error induced by process nonlinearity, a goal that the current work shares. Christophersen (2006) defined a general class of linear hybrid systems that includes the following sub-classes:

- Mixed logical dynamical systems;

- Linear complementary systems;

- Max-min-plus-scaling systems;

- Polyhedral piecewise affine systems or piecewise affine systems;

Bemporad and Morari (1999) introduced the mixed logical dynamical systems. In this framework, auxiliary variables are used to transform logical facts involving continuous variables into linear inequalities. These auxiliary variables are incorporated into the state-space and used to update the state vector, expressing relations that describe interdependent physical laws, logic rules, and operating constraints. Saturation functions, discrete inputs, qualitative outputs, bilinear systems and finite state machines can be modelled by this class, which also provides a multi-model approach by expressing nonlinear dynamic systems through combinational logic as piecewise linear timeinvariant dynamic systems. 
Another hybrid system class was introduced in the seminal paper Heemels et al. (2000), which used ideas from the theory of the linear complementarity systems (LCS). LCS is a hybrid dynamical system defined by a linear ordinary differential equation (ODE) involving an algebraic variable that is required to be a solution of a finitedimensional linear complementarity problem (LCP) (Cottle et al., 1992). The LCS is defined by a finite number of smooth ODEs, called modes, with transitions between the modes occurring along a state trajectory. One way to understand LCS is viewing it as a class of dynamical systems that switches between several operating modes. Within each mode, an LCS behaves like a linear system.

Heemels et al. (2000) used LCP for the mode selection process. As for the determination of jumps, it was based on linear system theory, more specifically the geometric theory of linear systems. To obtain a solution of a complementarity system, the associated jumps of the state variables must be specified, i.e., the conditions under which a transition from one given mode to another given mode will take place must be precisely defined. The state spaces corresponding to different modes are not necessarily of the same dimension, but they are embedded in one encompassing space. Therefore, state trajectories may exhibit discontinuities when a mode switch takes place, as acknowledged by the authors.

De Schutter and Van Den Boom (2001) introduced a hybrid system class called max-min-plus-scaling systems in which discrete event systems that can be modelled using the operations maximisation, minimization, addition and scalar multiplication. These systems are extensions of max-plus linear systems (Baccelli et al., 1992; CuninghameGreen, 1979), which can be used to model discrete event systems with synchronisation but no choice. Introducing choice led to the appearance of the minimum operation, resulting in the max-min-plus systems. A further extension was obtained by adding scalar multiplication. This yielded max-min-plus-scaling (MMPS) systems, which is shown by the authors to encompass several other classes of discrete event systems such as maxplus-linear systems, bilinear max-plus systems, polynomial max-plus systems, separated max-min-plus systems and regular max-min-plus systems.

Finally, the Piecewise affine approximations are a class of linear hybrid systems that are used for approximating nonlinear systems using a multi-model approach (Heemels et al., 2001). They can capture nonlinearities by partitioning the state-input space into regions and associating with each region a different affine state update equation 
(Sontag, 1981). This paradigm is a powerful modelling tool that can capture general nonlinearities (e.g. by local approximation), constraints, saturations, switches, discrete inputs and states, and other hybrid modelling phenomena in dynamical systems.

Piecewise Affine (PWA) systems can approximate nonlinear systems via multiple linearisations at different operating points (OP) (Sontag, 1981; Ferrari-Trecate et al., 2003; Roll et al., 2004). The space is partitioned through a series of linear approximations. As the plant operating point moves throughout the state-input space the state update equation is changed according to the linear model valid locally, as shown in Fig. 1. The larger the number of partitions the closer the approximations becomes to the nonlinear model.

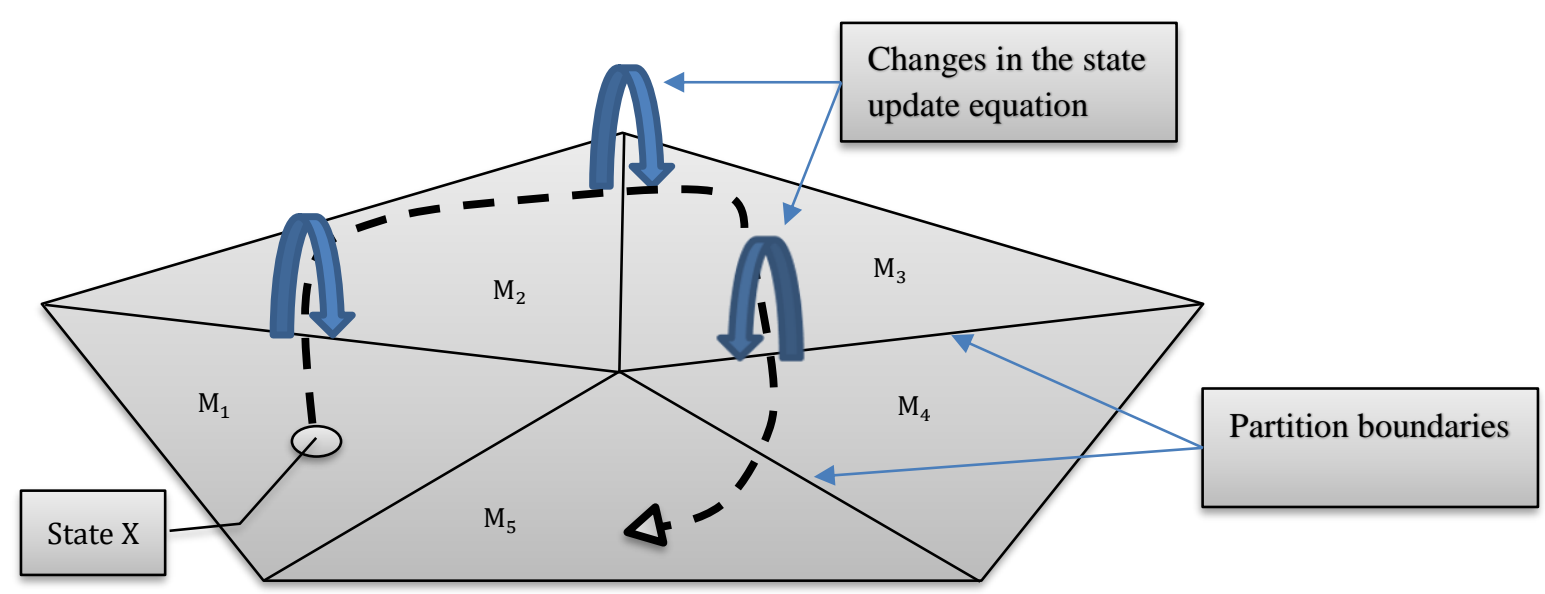

Fig. 1 - The operating point moves through the boundaries of a PWA system.

A literature review of switched and PWA models is presented in Roll (2003), and a tutorial paper in Paoletti et al. (2007). A survey on switched and piecewise affine system identification is presented in Garulli et al. (2012), from which the main approaches on the topic were presented. These main types of approaches were classified as optimisationbased, algebraic and recursive methods for Switched ARX (SARX) models, optimisationbased and clustering-based methods for PieceWise ARX (PWARX) models, and batch and recursive methods for state-space systems. According to Garulli et al. (2012), a common feature of the approaches, which is present in works such as Vidal et al. (2003), Roll et al. (2004), Ferrari-Trecate et al. (2003), Nakada et al. (2005), Juloski et al. (2005) and Bemporad et al. (2005), is that they lead to suboptimal solutions to the problem of inferring a PWARX model from data, while keeping an affordable computational burden.

Nevertheless, the linear hybrid systems framework has some issues that keep them from being the ideal solution for use with integrated process design and control 
approaches: firstly, the availability of the full set of equations of the nonlinear model is a requirement for most methods. The nonlinear model is linearised at specific points to generate several approximations, but this cannot be done if commercial simulation packages are to be used.

While it is relatively straightforward to employ a standard PWA approach to identify step response models, a good approximation of the nonlinear simulation package cannot be guaranteed. For instance, the "point-to-point" method (Lowe and Zohdy, 2010) which is used to identify optimal partitions for PWA systems depends on the availability of a full set of differential equations describing the process. If this condition is not met, then any partition definition will necessarily be arbitrary since it will be dependent on the starting point of the identification data. Both the choice of linearisation points and boundaries will not be optimal, and the PWA identification problem becomes finding the best fit to the available data. In this situation, the only way one could ensure that a modified PWA scheme would provide an adequate approximation would be by the use of numerous models valid for small regions, if data is available from several starting points.

This leads to the second issue: it is desirable to have a multi-model class capable of reliable and accurate prediction by using only a small number of sub-models. If the necessary number of partitions is high, the process of data-based model identification is very laborious and time-demanding. This is true when the data is provided by dynamic simulators but even more so when we desire to control an existing chemical plant.

Thirdly, the discontinuities at the boundaries of the regions can generate inconsistencies, with sudden changes in the output predictions that might introduce error in the plant assessment. For these reasons, the SMLP is presented as an alternative to PWA and other linear hybrid systems.

\subsection{Statistics-based multi-model methods}

The three issues presented at the end of the previous Section are addressed to a certain extent using what can be considered as a fifth class of linear hybrid systems, the statistics-based multi-model methods. Among them, the methods using Cluster analysis are very significant and thus highlighted in this Section. Cluster analysis is a technique that, in simple terms, attempts to divide a supposedly heterogeneous set of data into homogeneous groups or clusters. 
Using Cluster analysis to identify linear approximations of the nonlinear model is a route that has been used when the first-principles model is unknown or when it is desirable to simplify the numerical optimisation problem. The starting point is a set of data which may have been obtained from a real plant, simulators or from the full set of nonlinear equations when the latter is available. Ferrari-Trecate et al., 2003 presents a way of obtaining several optimised linearisations, where the states with similar characteristics are grouped into clusters and a linear model is identified for that region. Alternatively, an explicit expression for probability density of the data can be used (Nakada et al. 2005). The problem of representing the plant when the current operating point is detached from each cluster is addressed by Aumi and Mhaskar (2012), in which the linear approximations are combined using weights based on the proximity of the operating point to the models' "epicentre". The rules for shifting the weight of each model are given by weighting functions that arise from fuzzy c-means clustering. This method shares some similarity with the SMLP method presented in this paper, but unlike it, however, only one state is used, and the shift happens in the state update equation rather than the simultaneous states (see Section 3 for details).

According to Everitt (1972), the technique of cluster analysis is heavily beset with problems. The clustering problem is not well defined, since a cluster is not a well-defined concept, and it may be that no single definition is sufficient. Some methods of cluster analysis are better at finding clusters of a certain shape than others (for example most of the methods in common use, are good at finding clusters which are spherically distributed about their centres but are very bad at finding, for instance, long parallel clusters). However, as a priori, there is no reason to believe the clusters of a given process are more likely to be one shape or another, and thus no a priori reason for picking a particular clustering method to use on our data. This, of course, would be no problem if all the techniques of cluster analysis applied to the same set of data gave the same groups, but this is decidedly not the case, and, as shown by Everitt (1974), different techniques of cluster analysis applied to the same set of data may give very different results. Identifying the number of clusters is also a difficult task and merging overlapping clusters can result in wrong predictions (Agarwal, 2011). Hence, the clusters found are ultimately arbitrarily defined and there is, in practice, the problem of validating them, however reasonable they may seem to be to the experimenter. 
Given the interesting qualities of the statistics-based multi-model methods, what is the motivation for creating the SMLP? Unlike the techniques presented in this Section, the SMLP presented in the next Section requires no expert knowledge in statistics or fuzzy programming to be applied. The mathematics required is simple, and the resulting optimisation problems can be solved with available off-the-shelf solvers such as the Interior-Point algorithm (fmincon) available on MATLAB.

The engineering effort and computing time required to model complex systems is likely to be smaller using the SMLP as compared to statistics-based methods. While clustering algorithms are good at handling low-dimensional data such as data sets involving only two or three dimensions, finding clusters of data objects in a highdimensional space is challenging, especially considering that such data can be very sparse and highly skewed. According to Bellman (1961), data analysis techniques such as clustering must deal with the "curse of dimensionality", which, in general terms, is the widely observed phenomenon of working well at lower dimensions while often perform poorly as the dimensionality of the analysed data increases.

\section{The simultaneous multi-linear prediction (SMLP)}

In this Section, the novel multiple state-space model method, simultaneous multilinear prediction (SMLP), is presented. Its role is to reduce the nonlinearity-related error and avoid discontinuities between approximated regions. The SMLP avoids discontinuities by avoiding partitions and changes in the state update equation through the whole space of feasible operating points. Instead of a single state vector as found in linear hybrid systems such as PWA, the SMLP uses multiple sub-state vectors calculated simultaneously, one for each linear state-space sub-model. Note that since these model matrices do not share the same state vector, they need not possess the same dimensions (a requirement for PWA systems). In the SMLP, all sub-states to some degree contribute to the main output at all times, as shown in Fig. 2. 


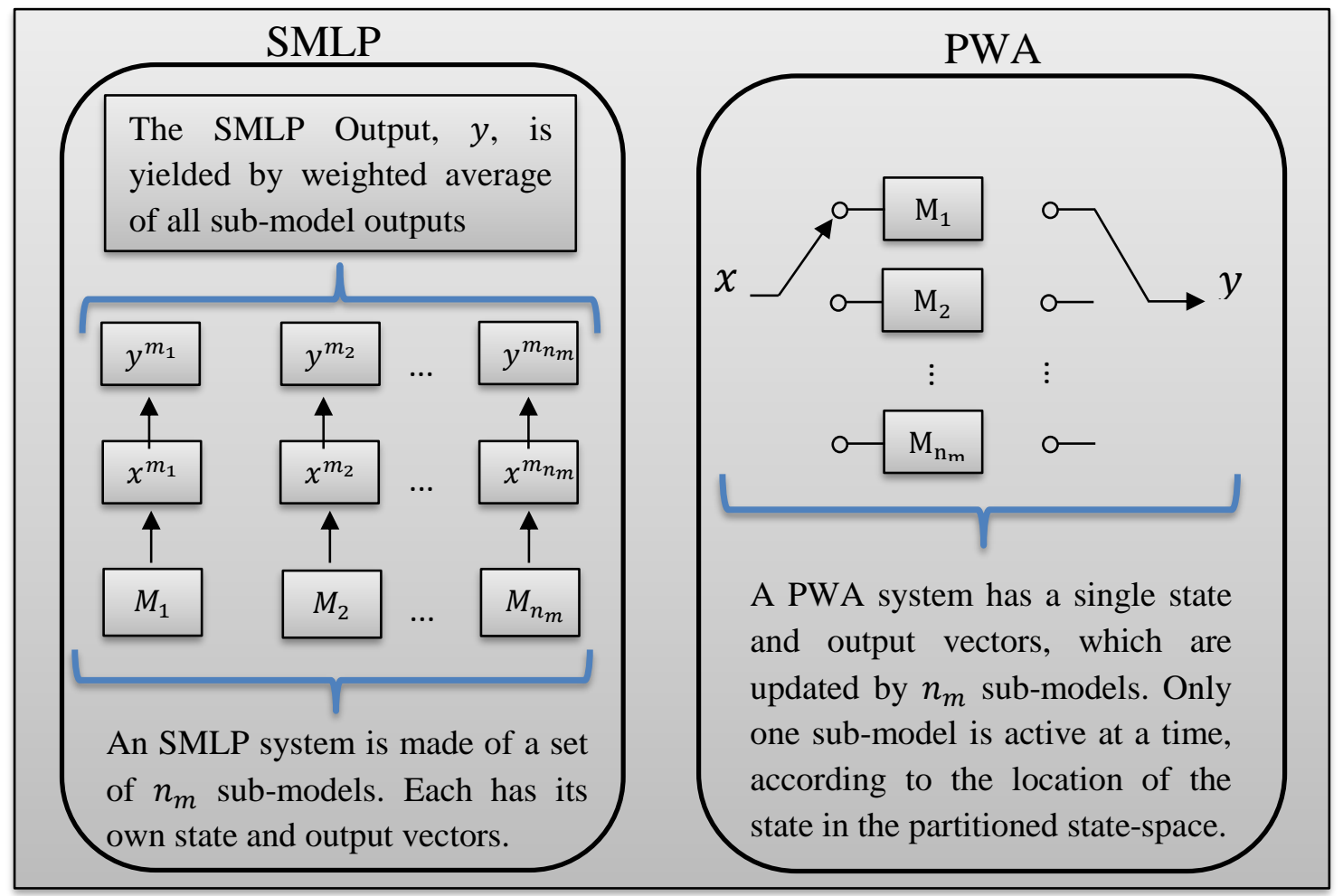

Fig. 2 - The output prediction can be generated by multiple simultaneous states, each one with its own update rule (SMLP system), or by a single state which continuously changes update rule (PWA system).

Each sub-state creates an output rate of change vector, and the result of the weighted addition of these vectors will generate the main output (or SMLP system output). The contribution ratio of each component is defined by a weight parameter, itself a function of three factors: the current position of the operating point; the points where each sub-model was originally identified (known as identification points or IPs); and a set of parameters obtained through linear regression in order to minimise the gap between the SMLP output and the simulator.

The SMLP does not assume that one particular sub-model has priority over others within an arbitrary partition of the input space. Without being restricted to any arbitrary bounds, the weight parameters change freely inside the whole space with a view to minimising the nonlinearity error. Besides avoiding discontinuities, eliminating partitions may provide a gain in accuracy since the search for optimal parameters becomes an unconstrained optimisation problem.

Three variants of the SMLP methodology have been devised by considering two different definitions of operating point (or OP) and two options for parametrisation obtained through regression analysis. In the first SMLP variant, the OP is the output (CV) vector (y), whereas for the other two variants the OP is the input vector $([\mathrm{u} \mathrm{d}])$ combining 
manipulated and disturbance variables (MVs and DVs). The first definition is appropriate when the CVs can be known precisely and updated regularly, and when there are no time delays or non-stationary (integrating) variables such as levels. On the other hand, OP can always be expressed as an MV vector since MVs are defined by the control system and are thus readily available. Measured disturbance variables (DVs) should also be incorporated in the OP.

As for the parametrisations, the two options consist of either having a single set of parameters for each multivariable sub-model, which will be called MIMO parametrisation or having a separated set for each pair of output/input, called SISO parametrisation, which may yield an improved prediction. A key disadvantage of the SISO (Single-Input Single-Output) parametrisation as compared to the MIMO (MultipleInputs Multiple-Outputs) parametrisation is the increased time required to perform the regression analysis, as it is individually done for each CV/MV or CV/DV pair. This tradeoff is significant if the number of variables is high.

\subsection{Generating sub-models}

The simultaneous multi-linear prediction is a method for obtaining a prediction for the future values of an output vector. Three main elements are used to provide this prediction: the initial state, the input profiles and a set of sub-models (linear approximations of a nonlinear model) identified at distinct states.

Each sub-model is to be obtained through model identification on a set of data generated by step increments on MVs and DVs. Alternatively, the relay response could be used. Tests may be performed on a dynamic simulator or on an experimental pilot plant, or even on a real industrial unit, for the sake of the methodology it is indifferent. Let us refer to the data thus obtained as the nonlinear model data, from which the submodels are derived and validated. The initial state from which a test is performed will be referred as an identification point (IP), and it will be considered the epicentre of the resulting sub-model. The greater the number of IPs/sub-models, the better the resilience of the resulting SMLP system regarding process nonlinearity. The control engineer should select IPs that are representative of the most common or critical operating conditions.

For each input/output couple, several values for the order of each sub-model should be tested, and the order adopted is that which minimises the numerical residue between the nonlinear model data and the sub-model being considered for all 
epicentres/IPs, not only the one being identified. This procedure avoids model overfitting or underfitting, which can have catastrophic results especially when the operating point (OP) is out of the vicinity of the model's IP. The model validation should be performed against a test data set for the SMLP system output and the output of each of its sub-models individually. In this paper, the identification data used was different from the validation data, and we consider this a good practice. The SMLP's parameters are chosen in order to reflect a range of conditions and thus the SMLP system is expected to exhibit smaller numerical residue in relation to both the identification and validation data than any of its constituent sub-models. The procedures to build an SMLP system are shown in Sections 3.2, 0 and 3.4.

\subsection{SMLP Method 1 - the MIMO parametrisation}

The Simultaneous Multi-Linear Prediction (SMLP) is a method for obtaining a prediction for the future values of an output vector. Three main elements are used to provide this prediction: the initial state, the input profiles and a set of sub-models (linear approximations of a nonlinear model) identified at distinct states.

Let us first outline the SMLP method for the special case of an output vector with two variables, $\boldsymbol{y}_{\mathbf{1}}$ and $\boldsymbol{y}_{2}$, which are to be controlled for a defined set of disturbances. Consider that, for a given flowsheet, model identification was performed at two identification points, $\quad \boldsymbol{I} \boldsymbol{P}_{\mathbf{1}}=\left[y_{1, I P_{1}}, y_{2, I P_{1}}\right] \quad$ and $\quad \boldsymbol{I} \boldsymbol{P}_{\mathbf{2}}=\left[y_{1, I P_{2}}, y_{2, I P_{2}}\right], \quad$ yielding respectively sub-models $\boldsymbol{M}_{\mathbf{1}}$ and $\boldsymbol{M}_{\mathbf{2}}$ that are Taylor approximations of the nonlinear model.

The SMLP formulation makes use of a standard linear state-space formulation presented in Lee et al. (1994). The sub-models $\boldsymbol{M}_{\mathbf{1}}$ and $\boldsymbol{M}_{\mathbf{2}}$ are systems of equations defined, respectively, by the state-space matrices $\boldsymbol{A}_{\boldsymbol{m}_{1}}, \boldsymbol{B}_{\boldsymbol{m}_{1}}, \boldsymbol{C}_{\boldsymbol{m}_{1}}$ and $\boldsymbol{D}_{\boldsymbol{m}_{1}}$ and $\boldsymbol{A}_{\boldsymbol{m}_{2}}$, $\boldsymbol{B}_{\boldsymbol{m}_{2}}, \boldsymbol{C}_{\boldsymbol{m}_{2}}$ and $\boldsymbol{D}_{\boldsymbol{m}_{2}}$, as well as by state vectors of appropriate dimensions, $\boldsymbol{x}_{\boldsymbol{k}}^{\boldsymbol{m}_{1}}$ and $\boldsymbol{x}_{\boldsymbol{k}}^{\boldsymbol{m}_{2}}$,

where $k$ is the time at initial conditions. The state vectors $\boldsymbol{x}_{k}^{\boldsymbol{m}_{1}}$ and $\boldsymbol{x}_{\boldsymbol{k}}^{\boldsymbol{m}_{\mathbf{2}}}$ are henceforth called simultaneous sub-states since they evolve independently from each other, being subject to the same set of control actions $\left(\boldsymbol{u}_{\boldsymbol{k}}, \boldsymbol{u}_{\boldsymbol{k}+\mathbf{1}}, \ldots, \boldsymbol{u}_{\boldsymbol{k}+\boldsymbol{m}}\right.$, where $m$ is the number of control actions) through the control horizon, and also to the same set of disturbances $\left(\boldsymbol{d}_{\boldsymbol{k}}, \boldsymbol{d}_{\boldsymbol{k}+1}, \ldots, \boldsymbol{d}_{\boldsymbol{k}+\boldsymbol{p}}\right.$, where $p$ is the length of the prediction) through the entirety of the prediction horizon. Hence, each sub-model has a sub-state update equation in the incremental form, as shown in Eq. 1 and Eq. 2: 


$$
\begin{aligned}
& x_{k+1}^{m_{1}}=A_{m_{1}} x_{k}^{m_{1}}+B_{m_{1}} \Delta u_{k}+D_{m_{1}} \Delta d_{k} \\
& x_{k+1}^{m_{2}}=A_{m_{2}} x_{k}^{m_{2}}+B_{m_{2}} \Delta u_{k}+D_{m_{2}} \Delta d_{k}
\end{aligned}
$$

Where $\Delta \boldsymbol{u}_{\boldsymbol{k}}=\left[\boldsymbol{u}_{\boldsymbol{k}}-\boldsymbol{u}_{\boldsymbol{k}-\mathbf{1}}\right]^{T}$ and $\Delta \boldsymbol{d}_{\boldsymbol{k}}=\left[\boldsymbol{u}_{\boldsymbol{k}}-\boldsymbol{u}_{\boldsymbol{k}-\mathbf{1}}\right]^{T}$. Therefore, even though the inputs are the same for both sub-models, different values for next-instant sub-states, $x_{k+1}^{m_{1}}$ and $x_{k+1}^{m_{2}}$, are obtained as well as different output predictions, respectively $\boldsymbol{y}_{k}^{m_{1}}$ and $\boldsymbol{y}_{\boldsymbol{k}}^{\boldsymbol{m}_{2}}$ for $\boldsymbol{M}_{\mathbf{1}}$ and $\boldsymbol{M}_{\mathbf{2}}$, as shown in Eq. 3 and Eq. 4 :

$$
\begin{aligned}
& y_{k}^{m_{1}}=C_{m_{1}} x_{k}^{m_{1}} \\
& y_{k}^{m_{2}}=C_{m_{2}} x_{k}^{m_{2}}
\end{aligned}
$$

Eq. 3

Eq. 4

Let us define the operating point (OP) at time $k$ as being the output vector of the multi-model system, i.e., $\boldsymbol{O} \boldsymbol{P}_{\boldsymbol{k}}=\left[y_{1, k}, y_{2, k}\right]$. Note that usually $\boldsymbol{O P}_{\boldsymbol{k}} \neq \boldsymbol{y}_{\boldsymbol{k}}^{\boldsymbol{m}_{\mathbf{1}}} \neq \boldsymbol{y}_{\boldsymbol{k}}^{\boldsymbol{m}_{\mathbf{2}}}$. Due to nonlinearity effects, the model $\boldsymbol{M}_{\mathbf{1}}$ is expected to get less accurate the further the operating point $\boldsymbol{O P}$ is removed from the first identification point $\boldsymbol{I} \boldsymbol{P}_{\mathbf{1}}$. In other words, if the plant is operated close to an identification point, its modelling error will probably be small. This should be valid as a general trend even if untrue at some points. So, it makes sense that the weights of $\boldsymbol{M}_{\mathbf{1}}$ and $\boldsymbol{M}_{\mathbf{2}}$ are roughly inversely proportional to, respectively, the distance in the Euclidean $n_{y}$-space between points $\boldsymbol{I P}_{\mathbf{1}}$ and $\boldsymbol{O} \boldsymbol{P}_{\boldsymbol{k}}\left(\overline{\boldsymbol{I} \boldsymbol{P}_{\mathbf{1}} \boldsymbol{O P} \boldsymbol{P}_{\boldsymbol{k}}}\right)$ and between $\boldsymbol{I} \boldsymbol{P}_{\mathbf{2}}$ and $\boldsymbol{O} \boldsymbol{P}_{\boldsymbol{k}},\left(\overline{\boldsymbol{I} \boldsymbol{P}_{\mathbf{2}} \boldsymbol{O P}}\right)$, where $n_{y}$ is the number of CVs $\left(n_{y}=2\right.$, in this case). This concept is shown graphically in Fig. 3:

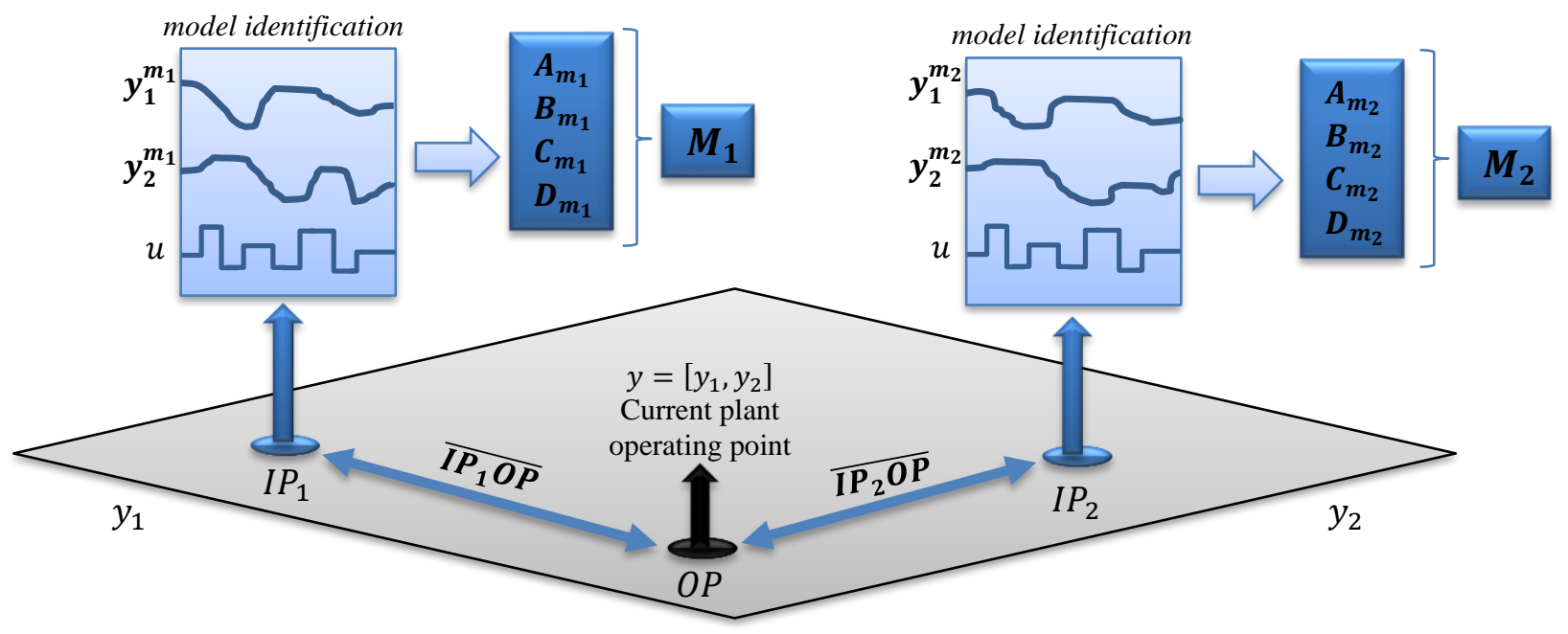

Fig. 3 - The vectors connecting the operating point $(\boldsymbol{O P})$ and $\boldsymbol{I P} \boldsymbol{1}_{\mathbf{1}}$ and $\boldsymbol{I} \boldsymbol{P}_{\mathbf{2}}$. 
Please note that obtaining more than one model for the same IP would make no sense, so we always assume $I \boldsymbol{P}_{1} \neq \boldsymbol{I} \boldsymbol{P}_{2}$. We define distance coefficients, $\tau_{1}$ and $\tau_{2}$, to represent the normalised proximity between $\boldsymbol{O} \boldsymbol{P}_{\boldsymbol{k}}, \boldsymbol{I} \boldsymbol{P}_{\mathbf{1}}$ and $\boldsymbol{I} \boldsymbol{P}_{\mathbf{2}}$.

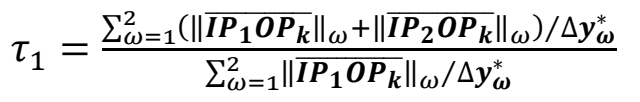

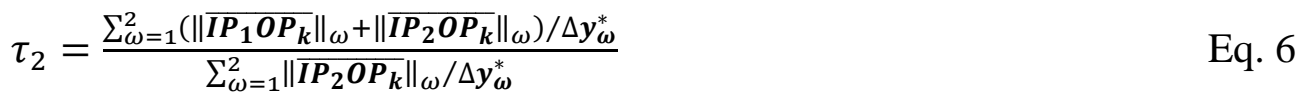

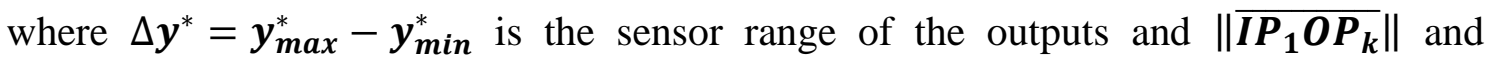

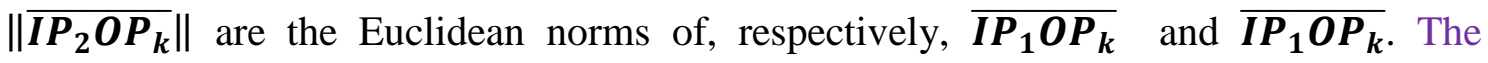
numerators of Eq. 5 and Eq. 6 both contain the sum of the Euclidean norms of the distances between the current operating point, $\boldsymbol{O P}_{\boldsymbol{k}}$, and the identification points of both

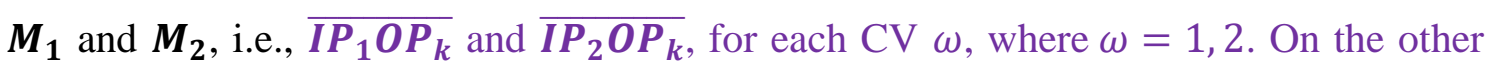
hand, the denominators of Eq. 5 and Eq. 6 contain, respectively, the sum of Euclidean

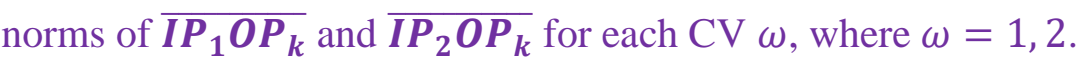

In exploring the dynamic performance, using these linearised models the SMLP method will search across the space covered by the two sub-models. As it crosses the boundaries instead of switching between models or upgrade equations here we calculate and update simultaneously the two output vectors and use a weighted arithmetic average to provide the combined model output prediction, $\boldsymbol{y}_{\boldsymbol{k}+\mathbf{1}}$. The weights of $\boldsymbol{M}_{\mathbf{1}}$ and $\boldsymbol{M}_{\mathbf{2}}$, denoted respectively as $\varepsilon_{1}$ and $\varepsilon_{2}$, are functions of the distance coefficients, $\tau_{1}$ and $\tau_{2}$. The format and coefficients of these functions should be selected as a means of minimising the error of the multi-model prediction as compared to a set of reference trajectories.

With this in mind, we introduce "degradation functions", $\boldsymbol{g}_{\mathbf{1}}$ and $\boldsymbol{g}_{\mathbf{2}}$, composed of positive, nonzero functions of the distance coefficients. They serve as nonlinear weighting parameters that will later be used to provide the averaged weights ( $\varepsilon_{1}$ and $\left.\varepsilon_{2}\right)$ of $\boldsymbol{M}_{\mathbf{1}}$ and $\boldsymbol{M}_{\mathbf{2}}$ in the main prediction. It is desirable that the degradation functions become roughly proportional to, respectively, $\tau_{1}$ and $\tau_{2}$, and with this in mind $\boldsymbol{g}_{\mathbf{1}}$ and $\boldsymbol{g}_{\mathbf{2}}$ may assume any format the user considers to be appropriate.

Finding a suitable format for the degradation function is a trial and error process, and there is no way to predict which one is going to yield minimum model mismatch. In brief, the format of the degradation functions must be decided on a case-by-case basis. 
The idea is to define the degradation functions so that a nonlinear component is added to an otherwise linear output update equation.

While searching for the optimised degradation functions one should follow standard procedures of data analysis, especially with regard to avoiding the overfitting phenomenon. Overfit occurs when a model is too strongly tailored to the particularities of the training set and generalizes poorly to new data. The "classic" way to avoid this is to use at least two dataset groups - a training set and a validation set. The best form of the equation and its coefficients are obtained using the training set; and the validation set is used to test for over-fitting (Gareth, 2013). Another method commonly used to avoid overfitting is to insert a Tikhonov regularisation term, which aims at measuring the complexity of the function. The higher the complexity, the higher the regularisation term will be (Tikhonov, 1963).

In this paper, $2^{\text {nd }}, 3^{\text {rd }}$ and $4^{\text {th }}$ order polynomials with non-negative coefficients were tested as degradation functions, as seen in Eq. 7 and Eq. 8. The coefficients were obtained using the same training dataset, and then the accuracy was tested by quantifying the residue between their predictions and the validation dataset.

The format of polynomials with non-negative coefficients is adequate since it avoids negative numbers and the averaged weight "degradation" decreases monotonically as the $\boldsymbol{O P}$ approaches a given $\boldsymbol{I P}$. Hence, a sub-model is dominant over others as a component of the SMLP in the proximities of its $\boldsymbol{I} \boldsymbol{P}$, e.g., $\boldsymbol{g}_{\mathbf{1}}\left(\tau_{1}\right) \gg \boldsymbol{g}_{2}\left(\tau_{2}\right)$ when $\boldsymbol{O P}_{\boldsymbol{k}} \rightarrow$ $\boldsymbol{I} \boldsymbol{P}_{\mathbf{1}}$, and $\boldsymbol{g}_{\mathbf{2}}\left(\tau_{2}\right) \gg \boldsymbol{g}_{\mathbf{1}}\left(\tau_{1}\right)$ when $\boldsymbol{O} \boldsymbol{P}_{\boldsymbol{k}} \rightarrow \boldsymbol{I} \boldsymbol{P}_{\mathbf{2}}$ (note that $\tau_{1} \rightarrow \infty$ and $\tau_{2} \rightarrow 1$ when $\overline{\boldsymbol{I \boldsymbol { P } _ { \mathbf { 1 } } \boldsymbol { O } \boldsymbol { P } _ { \boldsymbol { k } }}} \rightarrow[0,0]$. Similarly, $\tau_{2} \rightarrow \infty$ and $\tau_{1} \rightarrow 1$ when $\left.\overline{\boldsymbol{I \boldsymbol { P } _ { \mathbf { 2 } } \boldsymbol { O } \boldsymbol { P } _ { \boldsymbol { k } }}} \rightarrow[0,0]\right)$.

$$
\begin{aligned}
& \boldsymbol{g}_{\mathbf{1}}\left(\tau_{1}\right)=g_{1,4} \tau_{1}^{4}+g_{1,3} \tau_{1}^{3}+g_{1,2} \tau_{1}^{2}+g_{1,1} \tau_{1}+g_{1,0} \\
& \boldsymbol{g}_{2}\left(\tau_{2}\right)=g_{2,4} \tau_{2}^{4}+g_{2,3} \tau_{2}^{3}+g_{2,2} \tau_{2}^{2}+g_{2,1} \tau_{2}+g_{2,0}
\end{aligned}
$$

Evidently, $g_{1,4}=0$ if the degradation function is a $2^{\text {nd }}$ or $3^{\text {rd }}$ order polynomial, and $g_{1,3}=0$ if it is $2^{\text {nd }}$ order. In section 6.1, this number of coefficients was proved to be high enough to correctly capture the nonlinearity of the crude oil distillation plants presented in the case study of this paper.

Fig. 4 shows a possible visual interpretation of functions $\boldsymbol{g}_{\mathbf{1}}$ and $\boldsymbol{g}_{\mathbf{2}}$ : 


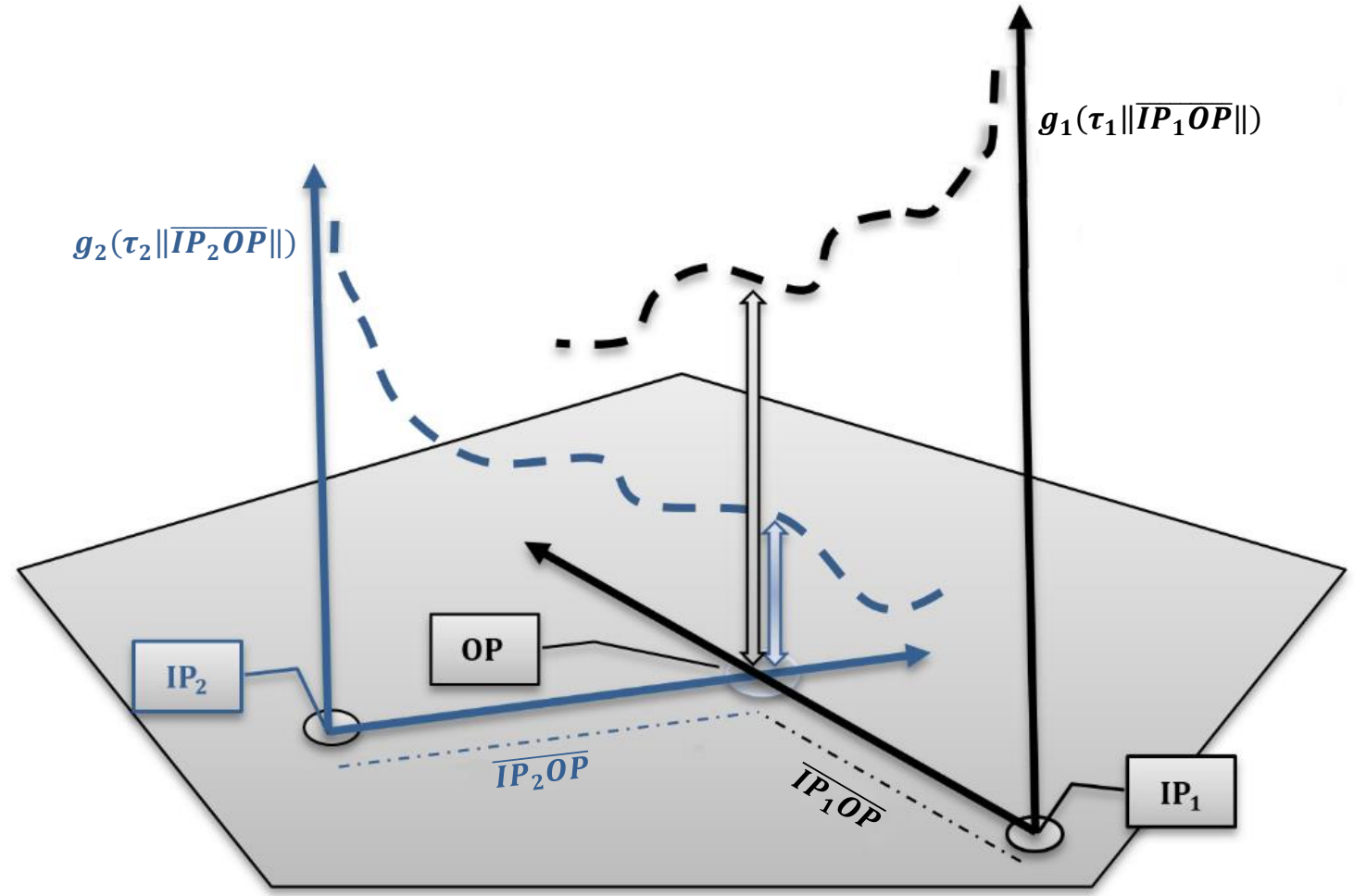

Fig. 4 - The "degradation functions", $\boldsymbol{g}_{1}$ and $\boldsymbol{g}_{2}$, are functions of the "distance coefficients" $\boldsymbol{\tau}_{\mathbf{1}}$ and $\boldsymbol{\tau}_{\mathbf{2}}$.

The averaged weights for the arithmetic average between sub-models, $\varepsilon_{1}$ and $\varepsilon_{2}$ are given by the proportion of $\boldsymbol{M}_{\mathbf{1}}$ and $\boldsymbol{M}_{\mathbf{2}}$ in the sum of the degradation functions, as per Eq. 9 and Eq. 10:

$$
\begin{aligned}
& \varepsilon_{1}=\frac{g_{1}\left(\tau_{1}\right)}{g_{1}\left(\tau_{1}\right)+g_{2}\left(\tau_{2}\right)} \\
& \varepsilon_{2}=\frac{g_{2}\left(\tau_{2}\right)}{g_{1}\left(\tau_{1}\right)+g_{2}\left(\tau_{2}\right)}
\end{aligned}
$$

Note that $\varepsilon_{1}+\varepsilon_{2}=1$. Finally, $\varepsilon_{1}$ and $\varepsilon_{2}$ are used in Eq. 11 to calculate the SMLP output at $(k+p)$. The SMLP output, which may also be called "main output" or "multimodel output" is normally the vector of CVs, but may sometimes contain other, noncontrolled variables that we wish to monitor. It must contain exactly the same variables as the sub-model outputs, i.e., $\operatorname{dim}\left(\boldsymbol{y}_{\boldsymbol{k}}\right)=\operatorname{dim}\left(\boldsymbol{y}_{\boldsymbol{k}}^{\boldsymbol{m}_{1}}\right)=\operatorname{dim}\left(\boldsymbol{y}_{\boldsymbol{k}}^{\boldsymbol{m}_{2}}\right)$. 


$$
\left[\boldsymbol{y}_{k+1}-\boldsymbol{y}_{k}\right]=\left[\varepsilon_{1, k+1}\left[\boldsymbol{y}_{k+1}^{\boldsymbol{m}_{1}}-\boldsymbol{y}_{k}^{\boldsymbol{m}_{1}}\right] \quad \varepsilon_{2, k+1}\left[\boldsymbol{y}_{k+1}^{\boldsymbol{m}_{2}}-\boldsymbol{y}_{k}^{\boldsymbol{m}_{2}}\right]\right]^{T}
$$

Note that the rate of change of $y_{k+1}$ is given by adding fractions $\left(\varepsilon_{1, k+1}\right.$ and $\left.\varepsilon_{2, k+1}\right)$ of the rates of change of $\boldsymbol{y}_{k+1}^{m_{1}}$ and $\boldsymbol{y}_{k+1}^{m_{2}}$, and no discontinuity is introduced in the prediction.

Now we will extend the multi-model approach to any number $n_{m}$ of linear submodels and any number $n_{y}$ of $\mathrm{CV}$, i.e., $\quad \boldsymbol{O P}_{\boldsymbol{k}}=\left[y_{1, k}, \ldots, y_{n_{y}, k}\right], \quad \boldsymbol{I} \boldsymbol{P}_{\mathbf{1}}=$ $\left[y_{1, I P_{1}}, \ldots, y_{n_{y}, I P_{1}}\right], \ldots, \boldsymbol{I} \boldsymbol{P}_{\boldsymbol{n}_{m}}=\left[y_{1, I P_{n_{m}}}, \ldots, y_{n_{y}, I P_{n_{m}}}\right]$. As before, there will be as many simultaneous sub-states and outputs as the number of sub-models, as shown in Eq. 12 and Eq. 13:

$$
\begin{aligned}
& x_{k+1}^{m_{1}}=A_{m_{1}} x_{k}^{m_{1}}+B_{m_{1}} \Delta u_{k}+D_{m_{1}} \Delta d_{k} \\
& \vdots \\
& x_{k+1}^{m_{n_{m}}}=A_{m_{n_{m}}} x_{k}^{m_{n_{m}}}+B_{m_{n_{m}}} \Delta u_{k}+D_{m_{n_{m}}} \Delta d_{k} \\
& y_{k}^{m_{1}}=C_{m_{1}} x_{k}^{m_{1}} \\
& \vdots \\
& y_{k}^{m_{n_{m}}}=C_{m_{n_{m}}} x_{k}^{m_{n_{m}}}
\end{aligned}
$$

Eq. 14 may be used to obtain the distance coefficients, $\tau_{\vartheta}$ :

$$
\tau_{\vartheta}=\frac{\sum_{\varphi=1}^{n_{m}} \sum_{\omega=1}^{n_{y}}\left\|\overline{\boldsymbol{I P}_{\boldsymbol{\varphi}} \boldsymbol{O \boldsymbol { P }} \boldsymbol{k}}\right\|_{\omega} / \Delta y_{\omega}^{*}}{\sum_{\omega=1}^{n_{y}}\left\|\overline{\boldsymbol{I \boldsymbol { P } _ { \vartheta } \boldsymbol { O } \boldsymbol { O P }}}\right\|_{\omega} / \Delta y_{\omega}^{*}}, \vartheta=1, \ldots, n_{m}
$$

Note that the demominator of Eq. 14 contains the sum of the Euclidean norms of the distances between the operating point $\boldsymbol{O} \boldsymbol{P}_{\boldsymbol{k}}$ and identification point $\boldsymbol{I} \boldsymbol{P}_{\boldsymbol{\vartheta}}\left(\overline{\boldsymbol{I} \boldsymbol{P}_{\vartheta} \boldsymbol{O} \boldsymbol{P}_{\boldsymbol{k}}}\right)$ of a particular sub-model $\boldsymbol{M}_{\boldsymbol{\vartheta}}$, for each $\mathrm{CV} \omega$, where $\omega=1, \ldots, n_{y}$. The numerator, however, contains the sum of the sum of Euclidean norms of the distances between $\boldsymbol{O} \boldsymbol{P}_{\boldsymbol{k}}$ and $\boldsymbol{I} \boldsymbol{P}_{\boldsymbol{\varphi}}\left(\overline{\boldsymbol{I P}_{\boldsymbol{\varphi}} \boldsymbol{O P _ { \boldsymbol { k } }}}\right)$ for each CV $\omega$, where $\omega=1, \ldots, n_{y}$, and each sub-model $\boldsymbol{M}_{\boldsymbol{\varphi}}$, where $\varphi=1, \ldots, n_{m}$. 
Of course, if $\left\|\overline{\boldsymbol{I \boldsymbol { P } _ { \boldsymbol { \vartheta } } \boldsymbol { O P }}{ }_{\boldsymbol{k}}}\right\|_{\omega}=0$ for some $\mathrm{CV} \omega$ and some model $\boldsymbol{\vartheta}$, this would generate infinite distance coefficients, causing the solver to crash. However, a simple contingency procedure was adopted consisting of adding a very small vector of appropriate dimensions to $\boldsymbol{O P}_{\boldsymbol{k}}$, such as $1^{-10} \cdot \underbrace{\left[\begin{array}{ll}1 & \ldots 1\end{array}\right]}_{1 \times n_{y}}$, if $\boldsymbol{I} \boldsymbol{P}_{\boldsymbol{\vartheta}}=\boldsymbol{O} \boldsymbol{P}_{\boldsymbol{k}}$. Using this procedure, which is standard in computer science, numeric errors could be easily avoided and the change to output prediction is insignificant.

The degradation function of sub-model $\boldsymbol{M}_{\boldsymbol{\vartheta}}$ is given by Eq. 15:

$$
\boldsymbol{g}_{\vartheta}\left(\tau_{\vartheta}\right)=g_{\vartheta, 4} \tau_{1}^{4}+g_{\vartheta, 3} \tau_{1}^{3}+g_{\vartheta, 2} \tau_{1}^{2}+g_{\vartheta, 1} \tau_{1}+g_{\vartheta, 0}, \vartheta=1, \ldots, n_{m} \quad \text { Eq. } 15
$$

The weight of sub-model $\boldsymbol{M}_{\vartheta}$ in the SMLP output prediction, $\varepsilon_{\vartheta}$, is then defined by Eq. 16:

$$
\varepsilon_{\vartheta}=\frac{g_{\vartheta}\left(\tau_{\vartheta}\right)}{\sum_{\varphi=1}^{n_{m}} g_{\varphi}\left(\tau_{\varphi}\right)}, \vartheta=1, \ldots, n_{m}
$$

Note that $\sum_{\vartheta=1}^{n_{m}} \varepsilon_{\vartheta}=1$. The SMLP output prediction can now be obtained through the use of Eq. 17:

$$
\left[\boldsymbol{y}_{\boldsymbol{k}+\mathbf{1}}-\boldsymbol{y}_{\boldsymbol{k}}\right]=\left[\sum_{\vartheta=1}^{n_{m}} \varepsilon_{\vartheta, k+1}\left[\boldsymbol{y}_{\boldsymbol{k}+\mathbf{1}}^{\boldsymbol{m}_{n_{\vartheta}}}-\boldsymbol{y}_{\boldsymbol{k}}^{\boldsymbol{m}_{\boldsymbol{n}}}\right]\right]
$$

A workflow showing the flux of data in this first variant of the SMLP method is presented in Fig. 5: 


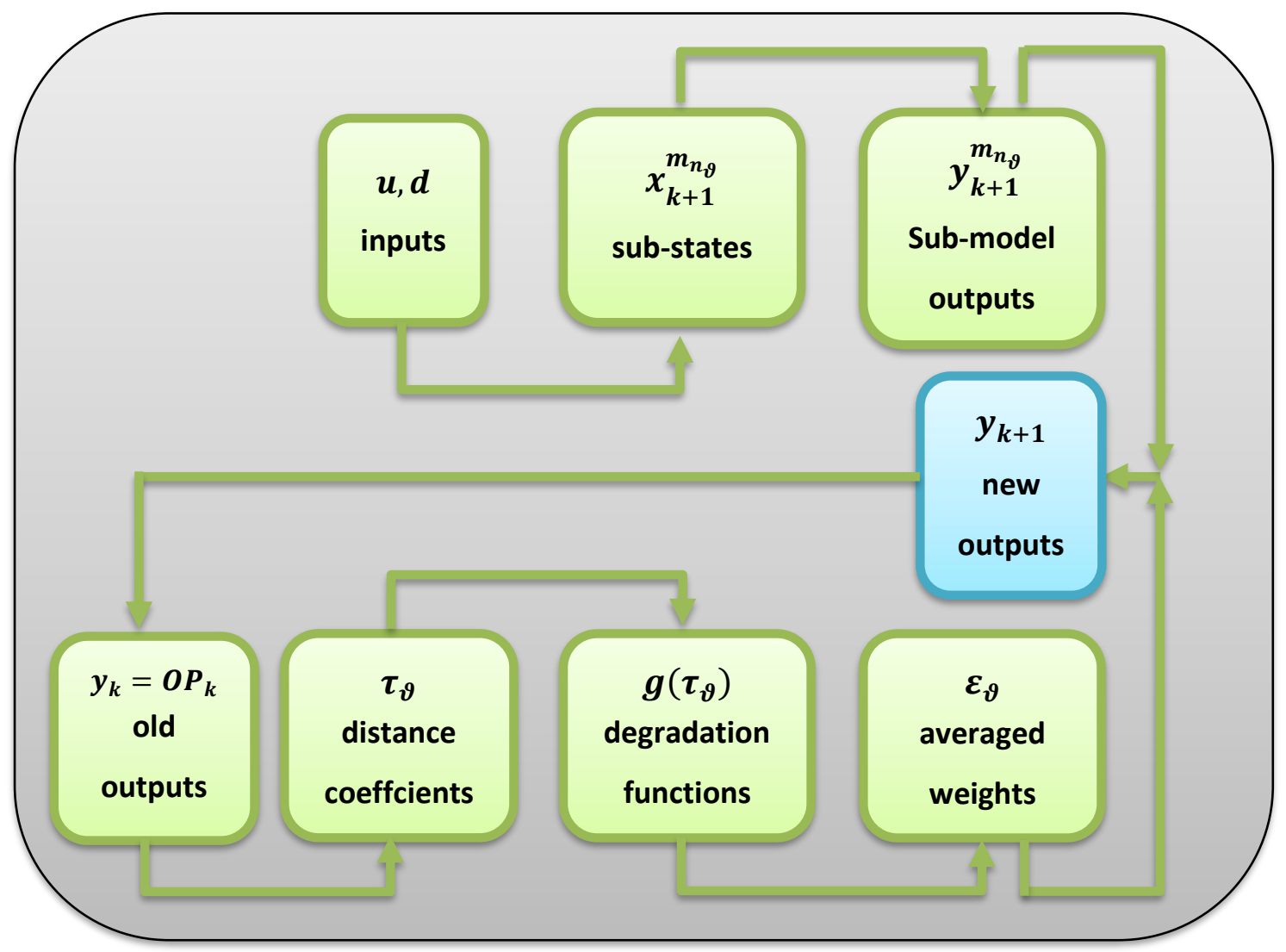

Fig. 5 - Data workflow of the first SMLP method. The distance coefficients are functions of the output, and the new prediction is provided by the multiplication of the sub-model outputs and the averaged weights.

In order to obtain the coefficients of the degradation functions $g$, we proceed with a regression analysis to minimise the normalised square error between the nonlinear model data (obtained from the commercial dynamic simulation package) and the SMLP, using the same inputs, through the entire prediction horizon. We recommend using several random trajectories and different starting points to properly identify the optimal constants through a range of scenarios. For this first method, an SMLP system can be defined as follows:

\section{Definition 3.2.1 Simultaneous Multi-Linear Prediction system with} MIMO parametrisation (OP defined as the output vector). Let there be a set of $n_{m}$ sub-models, where each sub-model $\boldsymbol{M}_{\boldsymbol{\vartheta}}$ is defined by the statespace matrices $\boldsymbol{A}_{\boldsymbol{m}_{\vartheta}}, \boldsymbol{B}_{\boldsymbol{m}_{\vartheta}}, \boldsymbol{C}_{\boldsymbol{m}_{\vartheta}}$ and $\boldsymbol{D}_{\boldsymbol{m}_{\vartheta}}$, a sub-state vector $\boldsymbol{x}_{\boldsymbol{k}}^{\boldsymbol{m}_{\vartheta}}$ and an identification point $\boldsymbol{I} \boldsymbol{P}_{\vartheta}=\left[y_{1, I P_{\vartheta}}, \ldots, y_{n_{y}, I P_{\vartheta}}\right]$, where $\vartheta=1, \ldots, n_{m}, n_{y}$ is the number of CVs and $k$ is an arbitrary initial time. Let the sub-state vectors for the next instant, $\boldsymbol{x}_{\boldsymbol{k}+\mathbf{1}}^{\boldsymbol{m}_{\vartheta}}$, be provided by Eq. 12 and Eq. 13. Let the vector 
of distance coefficients of sub-model $\boldsymbol{M}_{\boldsymbol{\vartheta}}, \boldsymbol{\tau}_{\boldsymbol{\vartheta}} \in \mathbb{R}^{n_{m}}$, be yielded by the ratio between $\sum_{\varphi=1, \ldots, n_{m}} \overline{\boldsymbol{I P _ { \varphi } \boldsymbol { O P }}}$ and $\overline{\boldsymbol{I P} \boldsymbol{P}_{\boldsymbol{\vartheta}} \boldsymbol{O P} \boldsymbol{k}}$ normalised for each $C V$, as defined in Eq. 14, where is the $\boldsymbol{O P}_{\boldsymbol{k}}$ operating point at time $k$, here defined as $\boldsymbol{O P}_{\boldsymbol{k}}=\left[y_{1, k}, \ldots, y_{n_{y}, k}\right]$, and $y_{k}$ is the main or multi-model output prediction at time $k$. Let the degradation function of sub-model $\boldsymbol{M}_{\boldsymbol{\vartheta}}, \boldsymbol{g}_{\boldsymbol{\vartheta}}\left(\boldsymbol{\tau}_{\boldsymbol{\vartheta}}\right)$, be defined by a set of non-negative coefficients $g_{\vartheta, 4}, g_{\vartheta, 3}, g_{\vartheta, 2}, g_{\vartheta, 1}$ and $g_{\vartheta, 0}$. Let the weight of the sub-model $\boldsymbol{M}_{\vartheta}$ in the main output prediction for each $C V, \varepsilon_{\vartheta}$, be defined as the ratio between $\boldsymbol{g}_{\boldsymbol{\vartheta}}\left(\boldsymbol{\tau}_{\boldsymbol{\vartheta}}\right)$ and $\sum_{\varphi=1}^{n_{m}} \boldsymbol{g}_{\boldsymbol{\varphi}}\left(\boldsymbol{\tau}_{\boldsymbol{\varphi}}\right)$. For such a system, $\boldsymbol{y}_{\boldsymbol{k}+\mathbf{1}}$ can be obtained through the use of Eq. 17, and the degradation function coefficients are obtained by solving the regression analysis problem given by Eq. 18 for a number $n_{t}$ of trajectories.

$$
\min _{g_{\vartheta, 4}, g_{\vartheta, 3}, g_{\vartheta, 2}, g_{\vartheta, 1}, g_{\vartheta, 0}} \sum_{\vartheta} \sum_{\eta} \sum_{\alpha}\left(\left[\boldsymbol{y}_{\eta, \boldsymbol{\alpha}}^{\boldsymbol{n} \boldsymbol{l}}-\boldsymbol{y}_{\boldsymbol{\eta}, \boldsymbol{\alpha}}^{\boldsymbol{m}_{\boldsymbol{n}}}\right] \operatorname{diag}\left|\boldsymbol{y}_{\max }^{*}-\boldsymbol{y}_{\min }^{*}\right|^{-1}\right)^{2} \quad \text { Eq. } 18
$$

Where $\boldsymbol{y}^{\boldsymbol{n} \boldsymbol{l}}$ is the training dataset provided by the dynamic simulator concerning the trajectories being tested, $\vartheta=1, \ldots, n_{m} ; \alpha=1, \ldots, p ; \eta=1, \ldots, n_{t}$, and $n_{t}$ is the number of reference trajectories that makes up the validation data set. Thus, the regression analysis parameters assume values that reduce as much as possible the prediction mismatch between the commercial dynamic simulation package and the multimodel prediction. This provides us with a high degree of information about the process, opening the "black box" of the commercial simulator and providing a proper basis for the EMOP analysis. The regression analysis problem of Eq. 18 is demanding computationally for large MIMO systems and large datasets, so it cannot be done continuously. If should be performed again every time the plant has changed, alongside with sub-model identification, at least.

As a reference to measure the performance of the SMLP, let us define in a similar way the linearisation error for a single sub-model $\boldsymbol{M}_{\boldsymbol{\vartheta}}$. The traditional single model approach is the equivalent of setting the degradation function of sub-model $\boldsymbol{M}_{\vartheta}$ to $\varepsilon_{\vartheta}=$ 1, thus ignoring the other sub-models, yielding Eq. 19: 


$$
\underset{\varepsilon_{\vartheta}=1}{E_{l i n}^{\vartheta}}=\sum_{\eta} \sum_{\alpha}\left(\left[\boldsymbol{y}_{\eta, \alpha}^{n l}-\boldsymbol{y}_{\eta, \boldsymbol{\alpha}}^{\boldsymbol{m}_{\boldsymbol{n}_{\vartheta}}}\right] \operatorname{diag}\left|\boldsymbol{y}_{\max }^{*}-\boldsymbol{y}_{\min }^{*}\right|^{-1}\right)^{2}
$$

The formulation presented in this section for an output-tracking SMLP system was defined with a view to avoiding the disadvantages of PWA systems presented in section 2. The goal of the SMLP is enabling a good approximation of a nonlinear plant using a small number of sub-models obtained at arbitrarily defined identification points, while altogether avoiding the need of partitioning the state-space and the inherent discontinuities thus introduced at the boundaries. Key to this formulation is the use of "degradation functions" that are defined to be continuous over the entire state-space, to shift smoothly the weight of each sub-model in the SMLP output. The most important step of the SMLP methodology is performing a regression analysis (Eq. 18) to find adequate format and parameters for the degradation functions. Having an optimised set of degradation functions results that, at any given point of the state-space, the SMLP output will tend to follow the sub-models that perform best in that region, while ignoring sub-models that perform poorly there, yielding a closer approximation to the nonlinear plant through the state-space covered by the test and validation data. The more comprehensive the data, the wider the range of states where the prediction is optimised.

An infinite number of states can be associated with a single OP. For this reason, the degradation functions are defined over the OP space instead of over the space-state: obtaining optimal coefficients for a degradation function defined over the space-state would very likely be impossible given the issue of state multiplicity. Moreover, OP concept is more intuitive, being widely applied in the chemical industry at large. In industrial operations, operators refer to operating points, not states.

\subsection{SMLP Method 2 - Operating point (OP) defined as the input vector}

In the previous Section, we measured the distance between OP and IPs using the output values, which are linearly related to the sub-states. Now another possibility is presented: defining $\boldsymbol{O P}$ and $\boldsymbol{I P}$ as sets of MVs and DVs. In this case, $\boldsymbol{O} \boldsymbol{P}_{\boldsymbol{k}}=$ $\left[u_{1, k}, \ldots, u_{n_{u}, k}, d_{1, k}, \ldots, d_{n_{d}, k}\right], \quad \boldsymbol{I} \boldsymbol{P}_{\mathbf{1}}=\left[u_{1, I P_{1}}, \ldots, u_{n_{u}, I P_{1}}, d_{1, I P_{1}}, \ldots, d_{n_{d} I P_{1}}\right], \ldots, \quad \boldsymbol{I} \boldsymbol{P}_{\boldsymbol{n}_{\boldsymbol{m}}}=$ $\left[u_{1, I P_{n_{m}}}, \ldots, u y_{n_{u}, I P_{n_{m}}}, d_{1, I P_{n_{m}}}, \ldots, d_{n_{d}, I P_{n_{m}}}\right]$, where $n_{d}$ is the number of DVs. Eq. 14 is thus updated to provide the new distance coefficients, $\tau_{\vartheta}$ : 


$$
\tau_{\vartheta}=\frac{\sum_{\varphi=1}^{n_{m}} \sum_{\omega}^{n_{u}+n_{d}}\left\|\overline{\boldsymbol{I P}_{\boldsymbol{\varphi}} \boldsymbol{O O \boldsymbol { P } _ { \boldsymbol { k } }}}\right\|_{\omega} /[\Delta \boldsymbol{u} \Delta \boldsymbol{d}]_{\omega}}{\sum_{\omega}^{n_{u}+n_{d}}\left\|\overline{\boldsymbol{I P}_{\vartheta} \boldsymbol{O} \boldsymbol{P}_{\boldsymbol{k}}}\right\|_{\omega} /[\Delta \boldsymbol{u} \Delta \boldsymbol{d}]_{\omega}}
$$

Where $\Delta \boldsymbol{u}=\boldsymbol{u}_{\max }-\boldsymbol{u}_{\min }, u_{j, \max }>u_{j, \min }, j=1, \ldots, n_{u}, \Delta \boldsymbol{d}=\boldsymbol{d}_{\max }-\boldsymbol{d}_{\min }$, $d_{l, \max }>d_{l, \min }, l=1, \ldots, n_{d}$, and the Euclidean norm of the IP/OP distance is now normalised by the MV and DV ranges. The rest of the procedure to obtain the SMLP remains the same, but the parameters defining the degradation functions, $\boldsymbol{g}_{\boldsymbol{\vartheta}}\left(\tau_{\vartheta}\right)$, are different and thus another regression analysis has to be performed. Evidently, the coefficients for methods 1 and 2 are not interchangeable even if the regression analysis is defined by Eq. 18 for both.

Fig. 6 shows a workflow of the data flux in this second variant of the SMLP method.

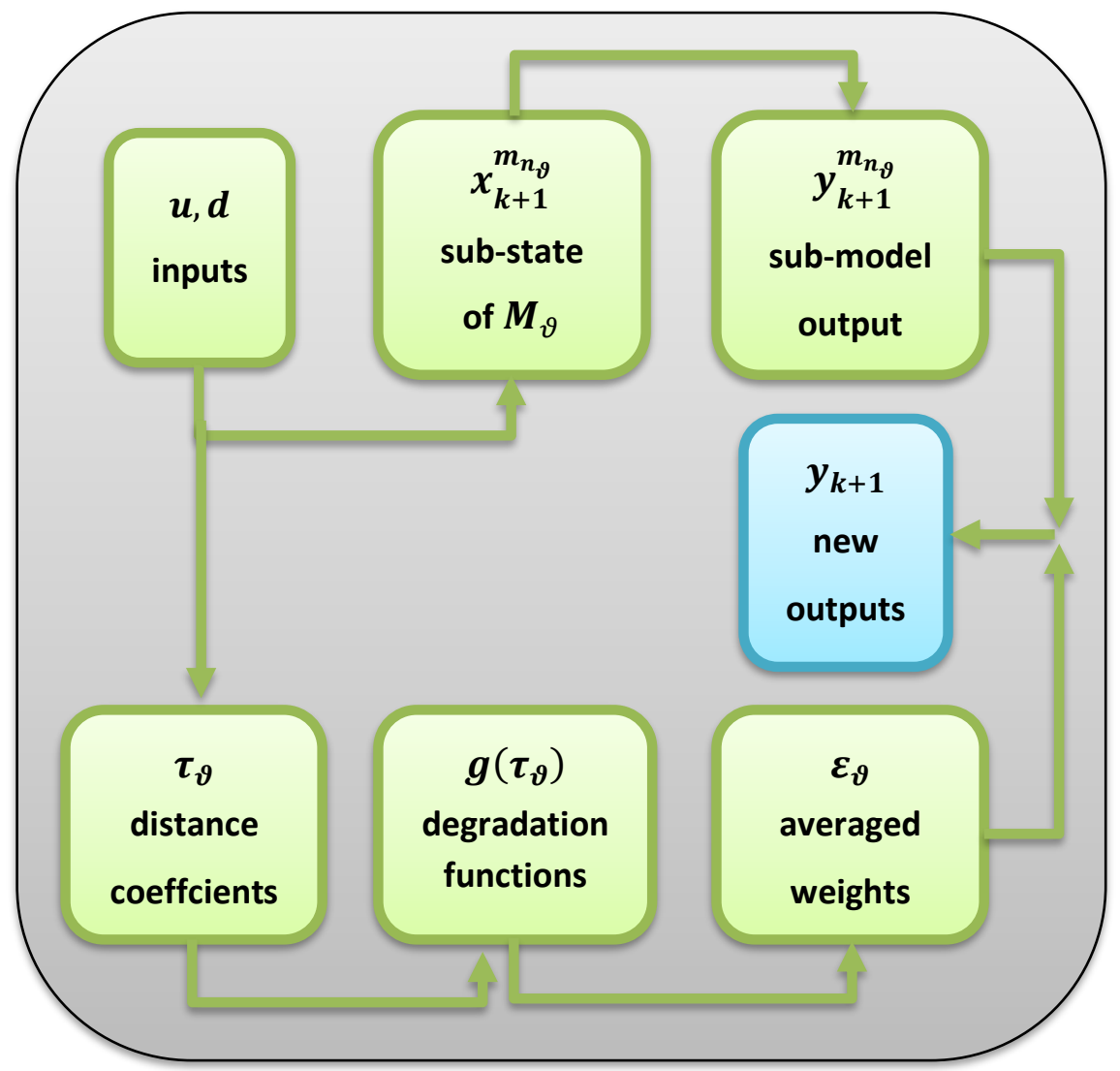

Fig. 6 - Data workflow of the second SMLP method. The distance coefficients are now functions of the input vector instead of the output vector.

For this second method, an SMLP system can be defined as follows: 


\section{Definition 3.4.1 Simultaneous Multi-Linear Prediction system with} MIMO parametrisation (OP defined as the input vector). Let there be $a$ set of $n_{m}$ sub-models, where each sub-model $\boldsymbol{M}_{\boldsymbol{\vartheta}}$ is defined by the statespace matrices $\boldsymbol{A}_{\boldsymbol{m}_{\vartheta}}, \boldsymbol{B}_{\boldsymbol{m}_{\vartheta}}, \boldsymbol{C}_{\boldsymbol{m}_{\vartheta}}$ and $\boldsymbol{D}_{\boldsymbol{m}_{\vartheta}}$, a sub-state vector $\boldsymbol{x}_{\boldsymbol{k}}^{\boldsymbol{m}_{\vartheta}}$ and an identification point $\boldsymbol{I P}_{\vartheta}=\left[u_{1, I P_{\vartheta}}, \ldots, u_{n_{u}, I P_{\vartheta}}, d_{1, I P_{\vartheta}}, \ldots, d_{n_{d}, I P_{\vartheta}}\right]$, where $\vartheta=1, \ldots, n_{m}, n_{u}$ is the number of MVs, $n_{d}$ is the number of DVs and $k$ is an arbitrary initial time. Let the sub-state vectors for the next instant, $\boldsymbol{x}_{\boldsymbol{k}+\mathbf{1}}^{\boldsymbol{m}_{\vartheta}}$, be provided by Eq. 12 and Eq. 13. Let the vector of distance coefficients of sub-model $\boldsymbol{M}_{\boldsymbol{\vartheta}}, \quad \boldsymbol{\tau}_{\boldsymbol{\vartheta}} \in \mathbb{R}^{n_{m}}$, be yielded by the ratio between $\sum_{\varphi=1, \ldots, n_{m}} \overline{\boldsymbol{I P} \boldsymbol{O P}_{\boldsymbol{k}}}$ and $\overline{\boldsymbol{I P}_{\boldsymbol{\vartheta}} \boldsymbol{O \boldsymbol { P } _ { \boldsymbol { k } }}}$ normalised for each $M V$, as defined in Eq. 20, where is the $\boldsymbol{O P} \boldsymbol{P}_{\boldsymbol{k}}$ operating point at time $k$, here defined as $\boldsymbol{O} \boldsymbol{P}_{\boldsymbol{k}}=$ $\left[u_{1, k}, \ldots, u_{n_{u}, k}, d_{1, k}, \ldots, d_{n_{d}, k}\right]$. Let the degradation function of sub-model $\boldsymbol{M}_{\vartheta}, \boldsymbol{g}_{\boldsymbol{\vartheta}}\left(\boldsymbol{\tau}_{\boldsymbol{\vartheta}}\right)$, be defined by a set of non-negative coefficients $g_{\vartheta, 4}, g_{\vartheta, 3}, g_{\vartheta, 2}$, $g_{\vartheta, 1}$ and $g_{\vartheta, 0}$. Let the weight of the sub-model $\boldsymbol{M}_{\boldsymbol{\vartheta}}$ in the main output prediction for each $C V, \varepsilon_{\vartheta}$, be defined as the ratio between $\boldsymbol{g}_{\boldsymbol{\vartheta}}\left(\boldsymbol{\tau}_{\boldsymbol{\vartheta}}\right)$ and $\sum_{\varphi=1}^{n_{m}} \boldsymbol{g}_{\boldsymbol{\varphi}}\left(\boldsymbol{\tau}_{\boldsymbol{\varphi}}\right)$. For such a system, the main or multi-model output prediction at time $k+1, \boldsymbol{y}_{\boldsymbol{k}+1}$ can be obtained through the use of Eq. 17, and the degradation function coefficients are obtained by solving the regression analysis problem given by Eq. 18 for a number $n_{t}$ of trajectories.

\subsection{SMLP Method 3 - the SISO parametrisation}

In previous Sections, the SMLP was based on the assumption that we can calculate a single weight $\varepsilon_{\vartheta}$ for each of the linear sub-model $\boldsymbol{M}_{\boldsymbol{\vartheta}}$, where $\vartheta=1, \ldots, n_{m}$, which dictates the overall contribution of model $\vartheta$. In this case, the contribution to the prediction calculation is, in relative terms, the same for all CVs and inputs. Let us return to the initial example of Section 3.1, where the multi-linear prediction was provided by two submodels, $\boldsymbol{M}_{\mathbf{1}}$ and $\boldsymbol{M}_{\mathbf{2}}$, and we had two CVs, $y_{1}$ and $y_{2}$. If, for instance, $\varepsilon_{1}>\varepsilon_{2}$, then submodel $\boldsymbol{M}_{\mathbf{1}}$ is the main contributor for the prediction calculation of both $y_{1}$ and $y_{2}$. However, maybe at current OP sub-model $\boldsymbol{M}_{\mathbf{1}}$ is adequate to calculate $\mathrm{y}_{1}$, while submodel $\boldsymbol{M}_{2}$ is better suited to provide $y_{2}$, or vice-versa. In this case, there may be some accuracy gain by defining a different $\varepsilon$ to each MV, for each sub-model. Let us carry out a few changes in the multi-linear prediction to reflect this concept. Eq. 20 is modified to 
provide the new distance coefficients, $\tau_{u_{j}}^{\vartheta}$ and $\tau_{d_{l}}^{\vartheta}$, where $j=1, \ldots, n_{u}, l=1, \ldots, n_{d}$ and $\vartheta=1, \ldots, n_{m}$.

$$
\begin{aligned}
& \tau_{u_{1}}^{\vartheta}=\frac{\sum_{\varphi=1}^{n_{m}}\left\|\overline{I P_{u_{1}, \varphi} O P_{u_{1}}}\right\| / \Delta u_{1}}{\left\|\overline{I P_{u_{1}, \vartheta} O P_{u_{1}}}\right\| / \Delta u_{1}} \\
& \text { ! } \\
& \tau_{u_{n_{u}}}^{\vartheta}=\frac{\sum_{\varphi=1}^{n_{m}}\left\|\overline{\overline{P_{u_{n_{u},}} O P_{u_{n_{u}}}}}\right\| / \Delta u_{n_{u}}}{\left\|\overline{I P_{u_{n_{u},}, \vartheta} O P_{u_{n_{u}}}}\right\| / \Delta u_{n_{u}}} \\
& \tau_{d_{1}}^{\vartheta}=\frac{\sum_{\varphi=1}^{n_{m}}\left\|\overline{I P_{d_{1}, \varphi} O P_{d_{1}}}\right\| / \Delta d_{1}}{\left\|\overline{I P_{d_{1}, \vartheta} O P_{d_{1}}}\right\| / \Delta d_{1}} \\
& \text { : } \\
& \tau_{d_{n_{d}}}^{\vartheta}=\frac{\sum_{\varphi=1}^{n_{m}}\left\|\overline{I P_{d_{n_{d}},} O P_{d_{n_{d}}}}\right\| / \Delta d_{n_{d}}}{\left\|\overline{I P_{d_{n}, \vartheta} O P_{n_{n_{d}}}}\right\| / \Delta d_{n_{d}}}
\end{aligned}
$$

The degradation functions should now reflect variable by variable input tracking, and there will now be one set of functions for the MVs, $\boldsymbol{g}_{\boldsymbol{u}_{\boldsymbol{j}}}^{\boldsymbol{\vartheta}}$, where $j=1, \ldots, n_{u}$, and another set for the DVs, $\boldsymbol{g}_{\boldsymbol{d}_{\boldsymbol{l}}}^{\boldsymbol{\vartheta}}$, where $l=1, \ldots, n_{d}$, and $\vartheta=1, \ldots, n_{m}$ for both sets. As before, these functions work as nonlinear weight parameters defining the weight of each sub-model $\boldsymbol{M}_{\vartheta}$ in the prediction of each $\mathrm{CV}$, but now the weight is also distinct for each input. Once more a $4^{\text {th }}$ order polynomial with non-negative coefficients is used as the format of the functions, as per Eq. 23 and Eq. 24:

$$
\begin{aligned}
& \boldsymbol{g}_{\boldsymbol{u}_{j}}^{\vartheta}\left(\tau_{u_{j}}^{\vartheta}\right)=g_{u_{j}}^{\vartheta, 4} \tau_{u_{j}}^{\vartheta}+g_{u_{j}}^{\vartheta, 3} \tau_{u_{j}}^{\vartheta^{3}}+g_{u_{j}}^{\vartheta, 2} \tau_{u_{j}}^{\vartheta^{2}}+g_{u_{j}}^{\vartheta, 1} \tau_{u_{j}}^{\vartheta}+g_{u_{j}}^{\vartheta, 0} \\
& \boldsymbol{g}_{\boldsymbol{d}_{l}}^{\vartheta}\left(\tau_{d_{l}}^{\vartheta}\right)=g_{d_{l}}^{\vartheta, 4} \tau_{d_{l}}^{\vartheta^{4}}+g_{d_{l}}^{\vartheta, 3} \tau_{d_{l}}^{\vartheta^{3}}+g_{d_{l}}^{\vartheta, 2} \tau_{d_{l}}^{\vartheta^{2}}+g_{d_{l}}^{\vartheta, 1} \tau_{d_{l}}^{\vartheta}+g_{d_{l}}^{\vartheta, 0}
\end{aligned}
$$

Eq. 16 is modified to reflect the fact that the weight parameters are in this case defined MV by MV and DV by DV, yielding Eq. 25 and Eq. 26: 


$$
\begin{aligned}
& \varepsilon_{u_{j}}^{\vartheta}=\frac{g_{u_{j}}^{\vartheta}\left(\tau_{u_{j}, \vartheta}\right)}{\sum_{\varphi=1}^{n_{m}} g_{u_{j}}^{\varphi}\left(\tau_{u_{j}, \varphi}\right)} \\
& \varepsilon_{d_{l}}^{\vartheta}=\frac{g_{d_{l}}^{\vartheta}\left(\tau_{d_{l}, \vartheta}\right)}{\sum_{\varphi=1}^{n_{m}} g_{d_{l}}^{\varphi}\left(\tau_{d_{l}, \varphi}\right)}
\end{aligned}
$$

The simultaneous sub-states are obtained from each linear sub-model $\boldsymbol{M}_{\boldsymbol{\vartheta}}$, where $\vartheta=1, \ldots, n_{m}$, and the weights $\boldsymbol{\varepsilon}_{\boldsymbol{d}}$ and $\boldsymbol{\varepsilon}_{\boldsymbol{u}}$, as shown in Eq. 27 to Eq. 29

$$
\begin{aligned}
& x_{k+1}^{m_{1}}=A_{m_{1}} x_{k}^{m_{1}}+B_{m_{1}} \varepsilon_{u, k}^{1} \Delta u_{k}+D_{m_{1}} \varepsilon_{d, k}^{1} \Delta d_{k} \\
& \vdots \\
& x_{k+1}^{m_{n_{m}}}=A_{m_{n_{m}}} x_{k}^{m_{n_{m}}}+B_{m_{n_{m}}} \varepsilon_{u, k}^{n_{m}} \Delta u_{k}+D_{m_{n_{m}}} \varepsilon_{d, k}^{n_{m}} \Delta d_{k} \\
& y_{k}^{m_{1}}=C_{m_{1}} x_{k}^{m_{1}} \\
& \vdots \\
& y_{k}^{m_{n_{m}}}=C_{m_{n_{m}}} x_{k}^{m_{n_{m}}} \\
& y_{k}=y_{k}^{m_{1}}+\cdots+y_{k}^{m_{n_{m}}}
\end{aligned}
$$

Note that when variable by variable input tracking is being performed, we are not applying the full inputs, $\Delta \boldsymbol{u}$ and $\Delta \boldsymbol{d}$, to all the linear sub-models, as in Sections 3.1 and 3.2. Instead, we split the inputs among the sub-models (Eq. 27) using $\boldsymbol{\varepsilon}_{\boldsymbol{d}}$ and $\boldsymbol{\varepsilon}_{\boldsymbol{u}}$, and sum the full resulting outputs (Eq. 29). The approach presented in this Section, henceforth called SMLP method 3, uses a larger number of regression analysis coefficients (3 . $\left.\left(n_{u}+n_{d}\right) \cdot n_{m}\right)$ as compared to method 1 and $2\left(3 \cdot n_{m}\right)$, and this extra information most likely increases the prediction's accuracy. Fig. 7 presents the data workflow of this third variant of the SMLP method. 


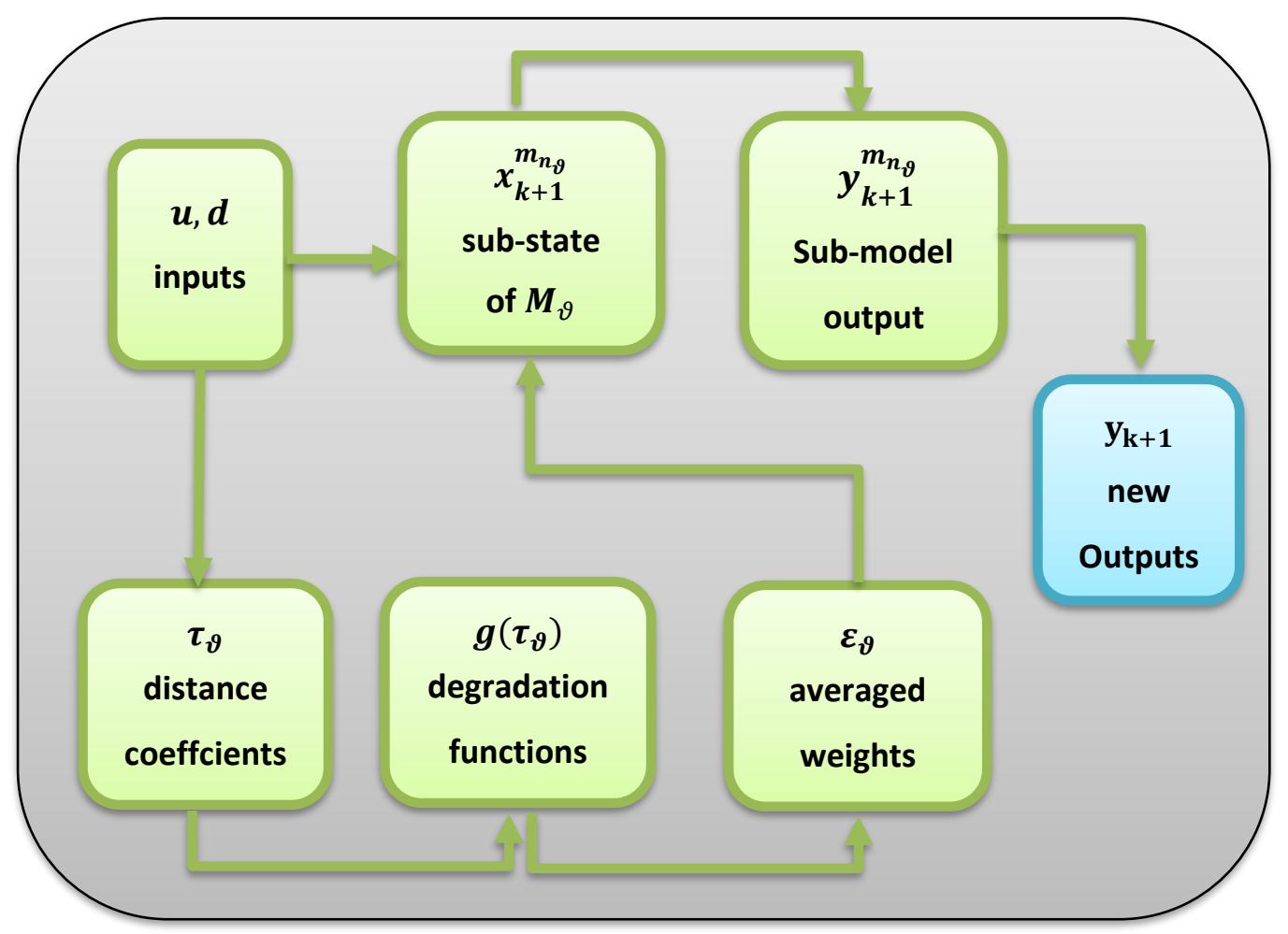

Fig. 7 - Data workflow of the third SMLP method. The averaged weights are now used to obtain the new sub-states.

A new regression analysis problem arises to obtain the coefficients of the

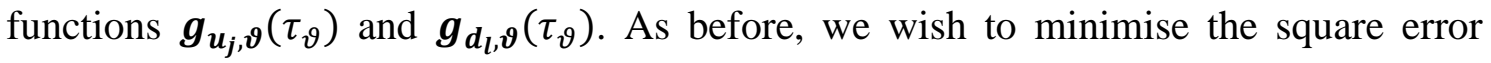
between the nonlinear model and the SMLP, using a random trajectory, through the entire prediction horizon.

For this third method, an SMLP system is defined as follows:

\section{Definition 3.4.1 Simultaneous Multi-Linear Prediction system with} SISO parametrisation (OP defined as the input vector). Let there be a set of $n_{m}$ sub-models, where each sub-model $\boldsymbol{M}_{\boldsymbol{\vartheta}}$ is defined by the statespace matrices $\boldsymbol{A}_{\boldsymbol{m}_{\vartheta}}, \boldsymbol{B}_{\boldsymbol{m}_{\vartheta}}, \boldsymbol{C}_{\boldsymbol{m}_{\vartheta}}$ and $\boldsymbol{D}_{\boldsymbol{m}_{\vartheta}}$, a sub-state vector $\boldsymbol{x}_{\boldsymbol{k}}^{\boldsymbol{m}_{\vartheta}}$ and an identification point $\boldsymbol{I} \boldsymbol{P}_{\vartheta}=\left[u_{1, I P_{\vartheta}}, \ldots, u_{n_{u}, I P_{\vartheta}}, d_{1, I P_{\vartheta}}, \ldots, d_{n_{d}, I P_{\vartheta}}\right]$, where $\vartheta=1, \ldots, n_{m}, n_{u}$ is the number of $M V s, n_{d}$ is the number of $D V s$ and $k$ is an arbitrary initial time. Let $\tau_{u_{j}}^{\vartheta}$ be the distance coefficient of sub-model $\boldsymbol{M}_{\boldsymbol{\vartheta}}$ related to the manipulated variable $j$, and $\tau_{d_{l}}^{\vartheta}$ be the distance coefficient of sub-model $\boldsymbol{M}_{\boldsymbol{\vartheta}}$ related to the disturbance variable $l$, where $j=1, \ldots, n_{u}, l=$ $1, \ldots, n_{d}$ and $\vartheta=1, \ldots, n_{m}$. Let $\tau_{u_{j}}^{\vartheta}$ be yielded by the ratio between 
$\sum_{\varphi=1}^{n_{m}}\left\|\overline{I P_{u_{\jmath}, \varphi} O P_{u_{\jmath}}}\right\|$ and $\left\|\overline{I P_{u_{\jmath}, \vartheta} O P_{u_{\jmath}}}\right\|$ normalised for each $M V$, and $\tau_{d_{l}}^{\vartheta}$ be yielded by the ratio between $\sum_{\varphi=1}^{n_{m}}\left\|\overline{I P_{d_{l}, \varphi} O P_{k, d_{l}}}\right\|$ and $\left\|\overline{I P_{d_{l}, \vartheta} O P_{d_{l}}}\right\|$ normalised for each DV, as defined in Eq. 21 and Eq. 22, where is the $\boldsymbol{O P}_{\boldsymbol{k}}$ operating point at time $k$, here defined as $\boldsymbol{O P}_{\boldsymbol{k}}=$ $\left[u_{1, k}, \ldots, u_{n_{u}, k}, d_{1, k}, \ldots, d_{n_{d}, k}\right]$. Let the degradation functions of sub-model $\boldsymbol{M}_{\boldsymbol{\vartheta}}$ related respectively to $u_{j}$ and $d_{l}, \boldsymbol{g}_{\boldsymbol{u}_{j}}^{\vartheta}\left(\tau_{u_{j}}^{\vartheta}\right)$ and $\boldsymbol{g}_{\boldsymbol{d}_{l}}^{\vartheta}\left(\tau_{d_{l}}^{\vartheta}\right)$, be defined by the sets of non-negative coefficients $g_{u_{j}}^{\vartheta, 4}, g_{u_{j}}^{\vartheta, 3}, g_{u_{j}, 2}^{\vartheta, g_{u_{j}}^{\vartheta, 1}}$ and $g_{u_{j}}^{\vartheta, 0}$ and $g_{d_{l}}^{\vartheta, 4}$, $g_{d_{l}}^{\vartheta, 3}, g_{d_{l}}^{\vartheta, 2}, g_{d_{l}}^{\vartheta, 1}$ and $g_{d_{l}}^{\vartheta, 0}$. Let the weights of the sub-model $\boldsymbol{M}_{\boldsymbol{\vartheta}}$ in the main output prediction for $u_{j}$ and $d_{l}, \varepsilon_{u_{j}}^{\vartheta}$ and $\varepsilon_{d_{l}}^{\vartheta}$, be defined respectively as the ratio between $g_{u_{j}}^{\vartheta}\left(\tau_{u_{j}, \vartheta}\right)$ and $\sum_{\varphi=1}^{n_{m}} g_{u_{j}}^{\varphi}\left(\tau_{u_{j}, \varphi}\right)$ and the ratio between $g_{d_{l}}^{\vartheta}\left(\tau_{d_{l}, \vartheta}\right)$ and $\sum_{\varphi=1}^{n_{m}} g_{d_{l}}^{\varphi}\left(\tau_{d_{l}, \varphi}\right)$. For such a system, the sub-state vectors for the next instant, $\boldsymbol{x}_{\boldsymbol{k}+\mathbf{1}}^{\vartheta}$, can be obtained through the use of Eq. 27 and Eq. 28. The main or multi-model output prediction at time $k+1, \boldsymbol{y}_{\boldsymbol{k}+\mathbf{1}}$, is given by Eq. 29, and the coefficients of the degradation functions are obtained by solving the regression analysis problem given by Eq. 30. This problem is defined for the variable by variable input tracking over a number $n_{t}$ of trajectories.

$$
\begin{aligned}
& \min _{g_{u_{j}}^{\vartheta, 4}, g_{u_{j}}^{\vartheta, 3}, g_{u_{j}, 2}^{\vartheta, 2}, g_{u_{j}}^{\vartheta, 1}, g_{u_{j}}^{\vartheta, 0}} \sum_{\vartheta} \sum_{\eta} \sum_{\alpha}\left(\left[\boldsymbol{y}_{\eta, \alpha}^{n l}-\boldsymbol{y}_{\eta, \alpha}^{m_{n_{\vartheta}}}\right] \operatorname{diag}\left|\boldsymbol{y}_{\max }^{*}-\boldsymbol{y}_{\min }^{*}\right|^{-1}\right)^{2} \\
& g_{d_{l}}^{\vartheta, 4}, g_{d_{l}}^{\vartheta, 3}, g_{d_{l}, 2}^{\vartheta, 2}, g_{d_{l}}^{\vartheta, 1}, g_{d_{l}, 0}^{\vartheta, 0}
\end{aligned}
$$

For Eq. 30, we have as before $j=1, \ldots, n_{u}, l=1, \ldots, n_{d}, \vartheta=1, \ldots, n_{m}, \alpha=$ $1, \ldots, p ; \eta=1, \ldots, n_{t}$.

\subsection{The stability of an SMLP system}

Concerning the stability of a SLMP system, the following theorem is valid:

Theorem 3.5.1 the stability of an SMLP system. An SMLP system is Lyapunov stable if and only if composed only of stable sub-models.

Proof. An SMLP system consists of the sum of a finite number of dynamic responses relative to each sub-model, each of which is multiplied by a nonnegative scalar, $\varepsilon$. If all sub-models are stable, the dynamic responses that 
make up the SMLP are going to be bound, and it is self-evident that the sum of a finite number of bounded dynamic responses is also bounded. The SMLP cannot make any of the individual responses unbounded. For instance, let us consider model state matrix $\boldsymbol{A}_{\boldsymbol{m}_{\boldsymbol{\vartheta}}}$ of sub-model $\boldsymbol{M}_{\boldsymbol{\vartheta}}$. From Lyapunov (1892), for continuous-time state-space formulations, if a model matrix $\boldsymbol{A}_{\boldsymbol{m}_{\vartheta}}$ has eigenvalues $\lambda_{\vartheta}$ with negative real parts, the system is BIBO (Bounded-Input Bounded-Output) stable. We have from the theorem of ESMM (Eigenvalues of a Scalar Multiple of a Matrix) that the eigenvalues $\varepsilon_{\vartheta} \lambda_{\vartheta}$ of matrix $\left[\varepsilon_{\vartheta} \boldsymbol{A}_{\boldsymbol{m}_{\vartheta}}\right]$ will also have negative real parts since $\varepsilon_{\vartheta} \in[0,1]$. For discrete-time formulations, a model is asymptotically (Schur) stable if and only if all the eigenvalues of its state matrix A have a magnitude less than one, i.e. lie inside the unit circle. Assuming that the sub-model $\boldsymbol{M}_{\boldsymbol{\vartheta}}$ is stable and thus $\left|\lambda_{\vartheta}\right| \in[0,1]$, it results in $\varepsilon_{\vartheta} \lambda_{\vartheta} \in[0,1]$, and therefore the response remains $B I B O$ stable.

Being Lyapunov Stable is a requirement for all flowsheets being evaluated through the use of the EMOP index (Strutzel and Bogle, 2016).

\subsection{Reachability of an SMLP system}

It is desirable to know if any given value for $x_{k}^{m_{n_{\vartheta}}}$ can be reached, i.e., if the system $\left(\boldsymbol{A}_{\boldsymbol{m}_{\vartheta}}, \boldsymbol{B}_{\boldsymbol{m}_{\vartheta}}\right)$ is completely reachable. Hence, let us focus on the problem, defined in Eq. 31 , of determining a sequence of $\mathrm{m}$ incremental control actions transferring the sub-state vector from $x_{k}^{m_{n_{\vartheta}}}=\bar{x}_{\vartheta, 1}$ to $x_{k+m}^{m_{n_{\vartheta}}}=\bar{x}_{\vartheta, 2}$.

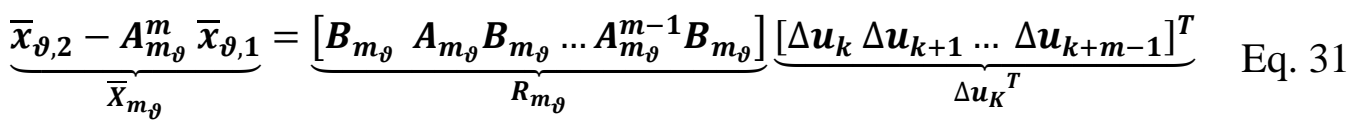

This is equivalent to solving the linear system of Eq. 32 with respect to $\Delta \mathrm{u}_{\mathrm{K}}$ :

$$
\boldsymbol{R}_{\boldsymbol{m}_{\vartheta}} \Delta \boldsymbol{u}_{K}^{T}=\bar{X}_{m_{\vartheta}}
$$

The matrix $\boldsymbol{R}_{\boldsymbol{m}_{\vartheta}} \in \mathbb{R}^{\boldsymbol{d}_{A, \vartheta} \boldsymbol{x} \boldsymbol{m} \cdot \boldsymbol{n}_{\boldsymbol{u}}}$ is called the reachability matrix of the subsystem. A solution $\Delta \boldsymbol{u}_{\boldsymbol{K}}$ to Eq. 32 exists if and only if $\overline{\boldsymbol{X}}_{\boldsymbol{m}_{\boldsymbol{v}}} \in \boldsymbol{I} \boldsymbol{I}\left(\boldsymbol{R}_{\boldsymbol{m}_{\boldsymbol{\vartheta}}}\right)$, where $\boldsymbol{I m}\left(\boldsymbol{R}_{\boldsymbol{m}_{\boldsymbol{v}}}\right)$ is the set of states that are reachable from the initial state $\overline{\boldsymbol{x}}_{\boldsymbol{\vartheta}, \mathbf{1}}$. According to the Rouché-Capelli 
theorem, this is true if $\operatorname{rank}\left(\boldsymbol{R}_{\boldsymbol{m}_{\vartheta}} \overline{\boldsymbol{X}}_{\boldsymbol{m}_{\vartheta}}\right)=\operatorname{rank}\left(\boldsymbol{R}_{\boldsymbol{m}_{\vartheta}}\right)$ and hence the following wellknown theorem is valid:

Theorem the complete reachability of a system (Kalman et al., 1969). The subsystem $\left(\boldsymbol{A}_{\boldsymbol{m}_{\boldsymbol{\vartheta}}}, \boldsymbol{B}_{\boldsymbol{m}_{\vartheta}}\right)$ is completely reachable if and only its reachability matrix is full rank, i.e., the largest rank possible for a matrix of the same dimensions

The full rank value is the lesser of the number of rows and columns and in this case $\operatorname{rank}\left(\boldsymbol{R}_{\boldsymbol{m}_{\vartheta}}\right)=m \cdot n_{u}$, where $\vartheta=1, \ldots, n_{m}$. A proof of the theorem is presented in Kalman et al. (1969). Let us now approach the multi-model reachability problem. The reachability matrices and the desired states of each sub-model can be concatenated yielding Eq. 33, the multi-model version of the system of Eq. 32. Note that the solution $\Delta \boldsymbol{u}_{\boldsymbol{K}}$ is the same as for Eq. 32 .

$$
\underbrace{\left[\begin{array}{c}
\boldsymbol{R}_{\boldsymbol{m}_{1}} \\
\vdots \\
\boldsymbol{R}_{m_{n_{m}}}
\end{array}\right]}_{\boldsymbol{R}_{M}} \Delta \boldsymbol{u}_{K}{ }^{T}=\underbrace{\left[\begin{array}{c}
\bar{X}_{m_{1}} \\
\vdots \\
\bar{X}_{m_{n_{m}}}
\end{array}\right]}_{\bar{X}_{M}}
$$

The theorem bellow is valid for SMLP systems:

Theorem 3.6.1 the complete reachability of an SMLP system. An SMLP system is completely reachable if and only if composed solely of completely reachable sub-models.

Proof. If an SMLP system is composed solely of completely reachable submodels, the reachability matrices of each sub-model $\boldsymbol{M}_{\boldsymbol{\vartheta}}, \boldsymbol{R}_{\boldsymbol{m}_{\boldsymbol{\vartheta}}}$, have full rank and the reachability matrix of the SMLP system, $\boldsymbol{R}_{\boldsymbol{M}}$, also has full rank. Since $\boldsymbol{R}_{\boldsymbol{M}}$ is the concatenation of the individual $\boldsymbol{R}_{\boldsymbol{m}_{\boldsymbol{\vartheta}}}$, where $\vartheta=$ $1, \ldots, n_{m}$, it results in $\operatorname{rank}\left(\boldsymbol{R}_{\boldsymbol{M}}\right)=\operatorname{rank}\left(\boldsymbol{R}_{\boldsymbol{m}_{\vartheta}}\right)=m \cdot n_{u}$. Likewise, $\boldsymbol{R}_{\boldsymbol{M}}$ will be rank deficient and the SMLP system will not be completely reachable if one or more sub-systems do not have full rank.

A completely reachable SMLP will be able to achieve any output value in the unrestricted control problem. In addition to that, it is shown in Hautus (1972) that controllability is a weaker condition than reachability, and that controllable systems are 
stabilisable. Therefore, if an SMLP is completely reachable it is also controllable and stabilisable.

\subsection{Filtering sub-state changes}

Due to the unavoidable presence of measurement noise and unmeasured disturbances in industrial, closed-loop MPC applications, the control engineer should implement a state-estimator, such as a Kalman Filter, for each sub-state of an SMLP system. The usual tuning considerations on this subject apply (Gelb, 1974). Please note that the regression analysis problems given by Eq. 18 and Eq. 30 are affected by the stateestimator tuning, and thus re-identification of the degradation functions is necessary if any of the estimators is changed. In this paper, state-estimators were not required since it was assumed that no measurement noise and no unmeasured disturbances were present in the case-studies.

\section{Comparison between two linear multi-model methods: Using the ASP as a case study to benchmark the simultaneous multi- linear prediction (SMLP)}

In this Section, an SMLP system is built for a case study provided in Francisco et al. (2011), with the goal of enabling comparison between it and a standard methodology.

It is desirable to know if the SLMP can approximate nonlinear models more accurately than the commonly employed piecewise affine (PWA) systems, i.e., if SLMP can further reduce error due to linearisation under frequent circumstances. With this goal in mind, the activated sludge process (ASP) case study will be used to benchmark the SLMP, by means of comparing its prediction to those generated by the nonlinear model presented in this chapter and by a standard PWA approach. The fact that the set of ODEs is available for the ASP, and that the model is relatively simple, makes it convenient to perform such a comparison.

The SMLP method is a particularly interesting option to consider when the nonlinear model is unknown, and the linear models must be obtained through model identification at arbitrary states. To emulate this typical use scenario, the model matrices (Eq. 62 to Eq. 65) were evaluated at three distinct, arbitrary states, yielding three linear models. These models were used to generate an SMLP and a PWA system. Since these systems consist of the same collection of linearised models and were provided with the 
same initial operating point (OP), MVs and DVs, differences in the predictions are the direct and sole result of differences in the methodologies themselves.

Unlike the case study of the atmospheric distillation plant of Section 6, whose dynamic data was provided by a commercial simulation package, a phenomenological nonlinear state-space model will be used to describe the activated sludge process (ASP) of a wastewater treatment plant. The low complexity of this case study, as compared to a full process unit, should help the reader to visualise some advantages of the SMLP approach.

\subsection{Description of an Activated Sludge Wastewater Treatment Plant}

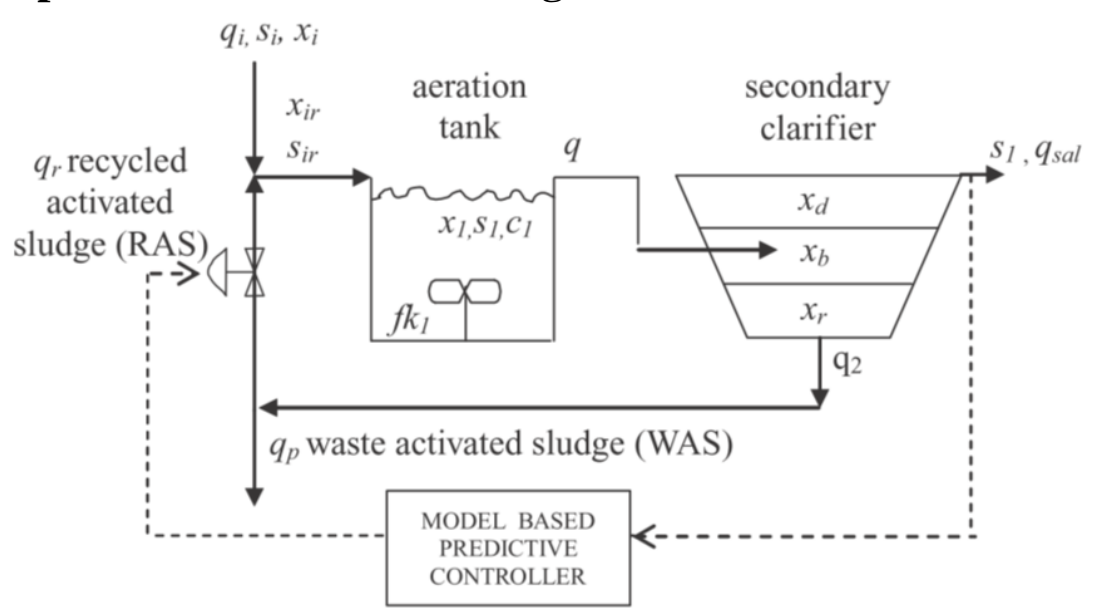

Fig. 8 - Plant and controller layout for the ASP for substrate elimination (Francisco et al. (2011))

A wastewater treatment plant is used to process sewage and return clean to a water body, and the activated sludge process (ASP) is a very important part of the cleaning procedure. The water treatment comprises the following basic steps, though in this Section only (b) and (c) are considered:

a) The primary treatment is dedicated to the removal of gross solids, sand, oil and grease. A primary sedimentation is the last step of this stage. This process removes up to $50 \%$ of the total polluting sewage load.

b) The secondary treatment is the ASP. The mixed outlet stream from the primary sedimentation tanks is passed to the reactor. There, the aerobic action of a mixture of microorganisms is used to reduce the substrate concentration in the water. A bacterial culture degrades the organic substrate converting it into inorganic products, more biomass and water. The dissolved oxygen required is provided by a set of aeration turbines. 
c) Clarification. The effluent is feed into clarification tanks, where the activated sludge and clean water are separated. After this, the water contains approximately $10 \%$ of the waste material, and the water is discharged to the river. Between $25 \%$ and $100 \%$ of the settled activated sludge is recycled to re-inoculate the reactor.

\subsubsection{Mathematical model of the ASP for substrate removal}

Now a simple model of ASP only for organic matter (substrate) elimination shall be described. The plant and controller layout can be seen in Fig. 8, comprising a bioreactor and a settler for clarification. The mathematical model assumes perfectly mixed tanks and is based on mass balances presented in Moreno et al. (1992).

\subsubsection{Mass balance for the aeration tanks}

The rate of change of the biomass, organic substrate and dissolved oxygen concentrations are given by:

$$
\begin{aligned}
& \frac{d x_{1}}{d t}=\mu_{\max } y_{c} \frac{s_{1} x_{1}}{\left(K_{S}+s_{1}\right)}-K_{d} \frac{x_{1}^{2}}{s_{1}}-K_{d} x_{1}+\frac{q}{V_{1}}\left(x_{i r}-x_{1}\right) \\
& \frac{d s_{1}}{d t}=-\mu_{\max } \frac{s_{1} x_{1}}{\left(K_{S}+s_{1}\right)}-f_{k d} K_{d} \frac{x_{1}^{2}}{s_{1}}+f_{k d} K_{c} x_{1}+\frac{q}{V_{1}}\left(s_{i r}-s_{1}\right) \\
& \frac{d c_{1}}{d t}=K_{l a} f_{k 1}\left(c_{s}-c_{1}\right)-O U R-\frac{q}{V_{1}} c_{1}
\end{aligned}
$$

where $x_{1}, s_{1}$ and $c_{1}$ are the biomass, substrate (Chemical Oxygen Demand, COD) and dissolved oxygen (DO) concentration at the output of the aeration tanks (mg/l); $x_{i r}$ and $s_{i r}$ are respectively the inlet biomass and substrate $(\mathrm{mg} / \mathrm{l}) . \mu_{\max }$ is the maximum growth rate of the microorganisms, $q$ is the inlet flow $\left(\mathrm{m}^{3} / \mathrm{h}\right), K_{d}$ is the kinetic coefficient of biomass decay by endogenous metabolism $(1 / \mathrm{h}), K_{c}$ is the kinetic coefficient of biomass decay by biological waste, $V_{1}$ is the total useful volume for the six aeration tanks $\left(\mathrm{m}^{3}\right), y_{c}$ is the yield coefficient between cellular growth and substrate elimination, $f_{k d}$ is the yield coefficient between biomass endogenous and substrate contribution to the medium, $c_{s}$ is the DO concentration at saturation, $K_{l a}$ is the mass transfer coefficient, $f_{k 1}$ is the aeration factor which depends on the number and speed of working turbines, OUR is the oxygen uptake rate and $K_{S}$ is the saturation constant.

For the biomass rate of change, the first term describes the biomass growth according to the Monod model, the second describes cell death, the third describes the 
biological waste, and the final term quantifies the dilution effects. For the rate of consumption of organic substrate, the first term expresses the decrease of the substrate through the activity of the biomass (Monod model), the second and third ones describe the transformation part of the dead biomass and biological waste into organic substrate, and the last term is the difference between the input and output substrate mass flow.

For the dissolved oxygen concentration, Eq. 35 follows the classic literature: the first term is the rate of oxygen transferred to the water, the second describes the rate of oxygen used by the microorganisms (uptake rate), and the final term quantifies the dilution effects. Algebraic equations for $x_{i r}$ and $s_{i r}$ are expressed as mass balances:

$$
\begin{aligned}
& x_{i r}=\frac{x_{i} q_{i}+x_{r} q_{r}}{q} \\
& s_{i r}=\frac{s_{i} q_{i}+s_{1} q_{r}}{q}
\end{aligned}
$$

where $x_{i}, s_{i}$ are the biomass and substrate at the influent, $q_{i}$ is the input flow to the process. $x_{r}$ and $q_{r}$ are the recycle concentrations and flow rate. The equation for oxygen uptake rate is:

$$
O U R=-K_{01} \mu_{\max } \frac{x_{1} s_{1}}{\left(K_{S}+s_{1}\right)}
$$

where $K_{01}$ is the yield coefficient between the cellular growth and the oxygen consumption rate.

\subsubsection{Mass balance for the secondary clarifiers (settlers)}

The operation of these elements is described by mass balance equations and one expression for the settling of activated sludge. The model considers the difference in settling rates between layers of increasing biomass concentration.

This model attempts to capture the dynamic behaviour of the clarifiers: 


$$
\begin{aligned}
& A_{s} l_{d} \frac{d x_{d}}{d t}=q_{s a l} x_{b}-q_{s a l} x_{d}-A_{s} v_{s}\left(x_{d}\right) \\
& A_{s} l_{b} \frac{d x_{b}}{d t}=q x_{1}-q_{s a l} x_{b}-q_{2} x_{b}+A_{s} v_{s}\left(x_{d}\right)-A_{s} v_{s}\left(x_{b}\right) \\
& A_{s} l_{r} \frac{d x_{r}}{d t}=q_{2} x_{b}-q_{2} x_{r}-A_{s} v_{s}\left(x_{b}\right)
\end{aligned}
$$

where $x_{d}$ is the biomass concentration at the surface of the settler leaving the plant, $q_{s a l}$ is the flow of clean water at the output of the settler, $x_{b}$ is the biomass concentration in the second layer, $q_{2}$ is the activated sludge total recycling flow, $x_{r}$ is the biomass concentration at the bottom of the settler, $v_{s}$ is the settling rate of the activated sludge, $A_{s}$ is the area of the settler, and $l_{d}, l_{b}, l_{r}$ are the height of the first, second and third layer, respectively (Fig. 8). Note that the settler input flow q enters to the unit at the second layer level. The settling rate parameters are fitted to a curve provided by pilot plants:

$$
\begin{aligned}
& v_{s}\left(x_{b}\right)=n r x_{b} e^{\left(-a r x_{b}\right)} \\
& v_{s}\left(x_{d}\right)=n r x_{d} e^{\left(-a r x_{d}\right)}
\end{aligned}
$$

The relations between the different flow rates are:

$$
\begin{aligned}
& q=q_{i}+q_{r} \\
& q_{s a l}=q_{i}-q_{p} \\
& q_{2}=q_{r}+q_{p}
\end{aligned}
$$

where $q_{p}$ is the purge flow. The control of this process aims to keep the substrate at the output $\left(s_{1}\right)$ below the legal requirement value despite the large variations of the flow rate $\left(q_{i}\right)$ and the substrate concentration of the incoming water $\left(s_{i}\right)$. The disturbances vector is: $\mathrm{d}=\left(s_{i}, q_{i}\right)$. The recycling flow $\left(q_{r}\right)$ is the MV, and the controlled output is the substrate $\left(s_{1}\right)$ in the reactor: $u(k)=q_{r} ; y(k)=s_{1}$. Biomass $\left(x_{1}\right)$ in the reactor is a bounded variable. 
Table 1 provides a symbol list for the variables and parameters of the activated sludge process, as well as their units and values when convenient. 
Table 1 - Operational, biological and physical parameters for the selected activated sludge process.

\begin{tabular}{|c|c|c|c|}
\hline Symbol & Parameter & Unit & Value \\
\hline$\mu_{\max }$ & Maximum growth rate of the microorganisms & $\mathrm{h}^{-1}$ & 0.1824 \\
\hline$y_{c}$ & Yield coefficient between cellular growth and substrate elimination & & 0.5948 \\
\hline$f_{k d}$ & $\begin{array}{l}\text { Yield coefficient between biomass endogenous and substrate contribution to the } \\
\text { medium }\end{array}$ & $\mathrm{L}^{-1}$ & 0.2 \\
\hline$K_{d}$ & Kinetic coefficient of biomass decay by endogenous metabolism & $\mathrm{L} / \mathrm{h}$ & $5.5 \mathrm{e}^{-5}$ \\
\hline$K_{s}$ & Saturation constant & $\mathrm{mg} / \mathrm{L}$ & 300 \\
\hline$K_{c}$ & Kinetic coefficient of biomass decay by biological waste & $\mathrm{L} / \mathrm{h}$ & $1.333 \mathrm{e}^{-4}$ \\
\hline$c_{s}$ & Saturation oxygen (DO) concentration in the aeration tanks & $\mathrm{mg} / \mathrm{L}$ & 8 \\
\hline$K_{l a}$ & Mass transfer coefficient in aeration process oxygen uptake rate & $\mathrm{h}^{-1}$ & 0.7 \\
\hline OUR & Oxygen uptake rate & $\mathrm{mg} / \mathrm{L} \cdot \mathrm{h}$ & (Variable) \\
\hline$K_{01}$ & Yield coefficient between the cellular growth and the oxygen consumption rate & & 0.0001 \\
\hline$x_{i}$ & Biomass concentration at the influent & $\mathrm{mg} / \mathrm{L}$ & (Variable) \\
\hline $\boldsymbol{s}_{\boldsymbol{i}}$ & Substrate concentration at the influent & $\mathrm{mg} / \mathrm{L}$ & (Variable) \\
\hline$q_{i}$ & Influent flow & $\mathrm{m}^{3} / \mathrm{h}$ & (Variable) \\
\hline$x_{1}$ & Biomass concentration at the output of the aeration tanks & $\mathrm{mg} / \mathrm{L}$ & (Variable) \\
\hline$s_{1}$ & Substrate (COD) concentration at the output of the aeration tanks & $\mathrm{mg} / \mathrm{L}$ & (Variable) \\
\hline$c_{1}$ & $\begin{array}{l}\text { Dissolved oxygen (DO) concentration at the output of the aeration tanks input } \\
\text { flow }\end{array}$ & $\mathrm{mg} / \mathrm{L}$ & (Variable) \\
\hline c & Bioreactor input flow & $\mathrm{m}^{3} / \mathrm{h}$ & (Variable) \\
\hline$q_{r}$ & Recycle flow & $\mathrm{m}^{3} / \mathrm{h}$ & (Variable) \\
\hline$q_{p}$ & Purge flow & $\mathrm{m}^{3} / \mathrm{h}$ & (Variable) \\
\hline$x_{i r}$ & Bioreactor inlet biomass concentration & $\mathrm{mg} / \mathrm{L}$ & (Variable) \\
\hline$s_{i r}$ & Bioreactor inlet substrate concentration & $\mathrm{mg} / \mathrm{L}$ & (Variable) \\
\hline$f_{k 1}$ & Aeration factor & & 0.15039 \\
\hline$V_{1}$ & Bioreactor volume & $\mathrm{m}^{3}$ & $\begin{array}{l}\text { (Depends on } \\
\text { flowsheet) }\end{array}$ \\
\hline$A_{s}$ & Settler area & $\mathrm{m}^{2}$ & $\begin{array}{l}\text { (Depends on } \\
\text { flowsheet) }\end{array}$ \\
\hline$x_{d}$ & Biomass concentration at the surface of the settler & $\mathrm{mg} / \mathrm{L}$ & (Variable) \\
\hline$x_{b}$ & Biomass concentration in the settler second layer & $\mathrm{mg} / \mathrm{L}$ & (Variable) \\
\hline$x_{r}$ & Biomass concentration at the bottom of the settler & $\mathrm{mg} / \mathrm{L}$ & (Variable) \\
\hline$v_{s}\left(x_{d}\right)$ & Settling rate function of the activated sludge in the settler depending on $\mathbf{x}_{\mathbf{d}}$ & $\mathrm{g} / \mathrm{m}^{2} \cdot \mathrm{h}$ & (Variable) \\
\hline$v_{s}\left(x_{b}\right)$ & Settling rate function of the activated sludge in the settler depending on $\mathrm{x}_{\mathrm{b}}$ & $\mathrm{g} / \mathrm{m}^{2} \cdot \mathrm{h}$ & (Variable) \\
\hline$n r$ & Settling rate experimental parameter & & 3.1563 \\
\hline$a r$ & Settling rate experimental parameter & $\mathrm{L} / \mathrm{mg}$ & $-7.8567 e^{-04}$ \\
\hline
\end{tabular}

\subsubsection{Nonlinear State-Space Model for the ASP}

Before applying the EMOP index methodology to the active sludge process, it is necessary first to obtain a state-space model of the process. Here we use a nonlinear statespace formulation detailed in Eq. 48 and Eq. 49 to provide the output prediction: 
where the letter $\delta$ was used in this Section instead of $\mathrm{x}$ to denote the states, thus avoiding confusion with some ASP variables. Let us now define the states, MVs and DVs of the MPC problem. It is important to point out that the variable scheme used in Francisco et al. (2011) is arguably not ideal, and the case study does not have the flexibility expected from an industrial process. For example, there is no feed tank, and thus the feed flow rate cannot be lowered to ensure on spec plant effluent, as it is common in situations such as this where heavy penalties can be imposed on companies who fail to meet environmental standards. For this reason, the plant flow rate will be considered a disturbance, whose variability may undermine the plant control goals. Additionally, there is no control of the purge flow rate in the original case study. In the next Section, we shall examine how the inclusion of purge flow as an MV enables better controllability, as the flowsheets shall be evaluated for both the cases of the fixed and variable purge.

So, unlike the control problem defined in Francisco et al. (2011), which considers only the recycling flow rate, $\mathrm{q}_{\mathrm{r}}$, as an $\mathrm{MV}$, we shall also set the purge flow rate, $\mathrm{q}_{\mathrm{p}}$, also as an MV. Table 2, Table 3 and Table 4 present respectively the model states, MVs and DVs to be used in the EMOP problem. The single CV is $y=\delta_{2}$ (substrate chemical oxygen demand).

Table 2 - States of the ASP model.

\begin{tabular}{llc}
\hline & \multicolumn{1}{c}{ ASP Model States } \\
\hline $\boldsymbol{\delta}_{\mathbf{1}}$ & $x_{1}$ & biomass COD (Chemical Oxygen Demand) $(\mathrm{mg} / \mathrm{L})$ \\
$\boldsymbol{\delta}_{\mathbf{2}}$ & $s_{1}$ & substrate COD (Chemical Oxygen Demand) $(\mathrm{mg} / \mathrm{L})$ \\
$\boldsymbol{\delta}_{\mathbf{3}}$ & $c_{1}$ & dissolved oxygen (DO) concentration at the output of the aeration tanks $(\mathrm{mg} / \mathrm{L})$ \\
$\boldsymbol{\delta}_{\mathbf{4}}$ & $x_{d}$ & biomass concentration at the surface of the settler $(\mathrm{mg} / \mathrm{L})$ \\
$\boldsymbol{\delta}_{\mathbf{5}}$ & $x_{b}$ & biomass concentration in the second layer $(\mathrm{mg} / \mathrm{L})$ \\
$\boldsymbol{\delta}_{\mathbf{6}}$ & $x_{r}$ & biomass concentration at the bottom of the settler $(\mathrm{mg} / \mathrm{L})$ \\
\hline
\end{tabular}

Table 3 - ASP Process inputs. ASP Model Inputs

\begin{tabular}{llc}
\hline $\boldsymbol{u}_{\mathbf{1}}$ & $q_{r}$ & recycling flow rate $\left(\mathrm{m}^{3} / \mathrm{h}\right)$ \\
$\boldsymbol{u}_{\mathbf{2}}$ & $q_{p}$ & purge flow rate $\left(\mathrm{m}^{3} / \mathrm{h}\right)$ \\
\hline
\end{tabular}

Table 4-ASP process disturbances. ASP Model Disturbances 


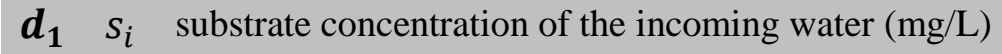

$\begin{array}{lll}\boldsymbol{d}_{\mathbf{2}} & q_{i} \quad \text { plant flow rate }\left(\mathrm{m}^{3} / \mathrm{h}\right)\end{array}$

Therefore, the states array $\delta=\left[\delta_{1}, \delta_{2}, \delta_{3}, \delta_{4}, \delta_{5}, \delta_{6}\right]$, the MV array $\boldsymbol{u}=\left[u_{1}, u_{2}\right]$ and the disturbance array $\boldsymbol{u}=\left[d_{1}, d_{2}\right]$ are defined.

Combining Eq. 34 to Eq. 47 and replacing the variables defined in Table 2 to Table 4 , yields the final form of the mass balance differential equations for the aeration tank and secondary clarifier, as presented in Eq. 50 to Eq. 55.

$$
\begin{aligned}
& f_{\delta_{1}}=\frac{d \delta_{1}}{d t}=\mu_{\max } y_{c} \frac{\delta_{2} \delta_{1}}{\left(K_{s}+\delta_{2}\right)}-K_{d} \frac{\delta_{1}^{2}}{\delta_{2}}-K_{c} \delta_{1}+\frac{1}{V_{1}}\left(x_{i} d_{2}+\delta_{6} u_{1}-\delta_{1} d_{2}-\right. \\
& \left.\delta_{1} u_{1}\right) \\
& f_{\delta_{2}}=\frac{d \delta_{2}}{d t}=-\mu_{\max } \frac{\delta_{2} \delta_{1}}{\left(K_{S}+\delta_{2}\right)}+f_{k d} \delta_{1}\left(K_{d} \frac{\delta_{1}}{\delta_{2}}+K_{c}\right)+\frac{d_{2}}{V_{1}}\left(d_{1}-\delta_{2}\right) \\
& f_{\delta_{3}}=\frac{d \delta_{3}}{d t}=K_{l a} f_{k 1}\left(c_{s}-\delta_{3}\right)+K_{01} \mu_{\max } \frac{\delta_{2} \delta_{1}}{\left(K_{s}+\delta_{2}\right)}-\frac{\delta_{3}}{V_{1}}\left(d_{2}+u_{1}\right) \\
& f_{\delta_{4}}=\frac{d \delta_{4}}{d t}=\frac{d_{2} \delta_{5}}{A_{s} l_{d}}-\frac{u_{2} \delta_{5}}{A_{s} l_{d}}-\frac{d_{2} \delta_{4}}{A_{s} l_{d}}+\frac{u_{2} \delta_{4}}{A_{s} l_{d}}-\frac{n r \delta_{4} e^{\left(-a r \delta_{4}\right)}}{l_{d}} \\
& f_{\delta_{5}}=\frac{d \delta_{5}}{d t}=\frac{d_{2} \delta_{1}}{A_{s} l_{b}}+\frac{u_{1} \delta_{1}}{A_{s} l_{b}}-\frac{d_{2} \delta_{5}}{A_{s} l_{b}}-\frac{u_{1} \delta_{5}}{A_{s} l_{b}}+\frac{n r \delta_{4} e^{\left(-a r \delta_{4}\right)}}{l_{b}}-\frac{n r \delta_{5} e^{\left(-a r \delta_{5}\right)}}{l_{b}} \\
& f_{\delta_{6}}=\frac{d \delta_{6}}{d t}=\frac{u_{1} \delta_{5}}{A_{s} l_{r}}+\frac{u_{2} \delta_{5}}{A_{s} l_{r}}-\frac{u_{1} \delta_{6}}{A_{s} l_{r}}-\frac{u_{2} \delta_{6}}{A_{s} l_{r}}+\frac{n r \delta_{5} e^{\left(-a r \delta_{5}\right)}}{l_{r}}
\end{aligned}
$$

The nonlinear state-space model matrices can be easily obtained by rearranging the differential equations of the ASP process, yielding the system described in Eq. 56 to Eq. 59:

$$
\boldsymbol{A}=\left[\begin{array}{c}
{\left[\mu_{\max } y_{c} \frac{\delta_{2}}{\left(K_{S}+\delta_{2}\right)}-K_{d} \frac{\delta_{1}}{\delta_{2}}-K_{c}\right]} \\
{\left[-\mu_{\max } \frac{\delta_{1}}{\left(K_{S}+\delta_{2}\right)}+\frac{f_{k d} \delta_{1}}{\delta_{2}}\left(K_{d} \frac{\delta_{1}}{\delta_{2}}+K_{c}\right)\right]} \\
{\left[K_{l a} f_{k 1}\left(\frac{c_{S}}{\delta_{3}}-1\right)+\frac{K_{01} \mu_{\max }}{\delta_{3}} \frac{\delta_{2} \delta_{1}}{\left(K_{S}+\delta_{2}\right)}\right]} \\
{\left[-\frac{n r e^{\left(-a r \delta_{4}\right)}}{l_{d}}\right]} \\
{\left[\frac{n r \delta_{4} e^{\left(-a r \delta_{4}\right)}}{\delta_{5} l_{b}}-\frac{n r e^{\left(-a r \delta_{5}\right)}}{l_{b}}\right]} \\
{\left[\frac{n r \delta_{5} e^{\left(-a r \delta_{5}\right)}}{\delta_{6} l_{r}}\right]}
\end{array}\right]
$$

Eq. 56 
$\boldsymbol{B}=\left[\begin{array}{cc}{\left[\frac{\delta_{6}-\delta_{1}}{V_{1}}\right]} & {[0]} \\ {[0]} & {[0]} \\ {\left[-\frac{\delta_{3}}{V_{1}}\right]} & {[0]} \\ {[0]} & {\left[\frac{\delta_{5}-\delta_{4}}{A_{s} l_{d}}\right]} \\ {\left[\frac{\delta_{1}-\delta_{5}}{A_{s} l_{b}}\right]} & {[0]} \\ {\left[\frac{\delta_{5}-\delta_{6}}{A_{s} l_{r}}\right]} & {\left[\frac{\delta_{5}-\delta_{6}}{A_{s} l_{r}}\right]}\end{array}\right]$

Eq. 57

$\boldsymbol{C}=\operatorname{diag}\left[\begin{array}{llllll}0 & 1 & 0 & 0 & 0 & 0\end{array}\right]$

Eq. 58

$\boldsymbol{D}=\left[\begin{array}{cc}{[0]} & {\left[\frac{x_{i}-\delta_{1}}{V_{1}}\right]} \\ {\left[\frac{q_{i}}{V_{1}}\right]} & {\left[-\frac{\delta_{2}}{V_{1}}\right]} \\ {[0]} & {\left[-\frac{\delta_{3}}{V_{1}}\right]} \\ {[0]} & {\left[\frac{\delta_{5}-\delta_{4}}{A_{s} l_{d}}\right]} \\ {[0]} & {\left[\frac{\delta_{1}-\delta_{5}}{A_{s} l_{b}}\right]} \\ {[0]} & {[0]}\end{array}\right]$

Eq. 59

\subsubsection{A linearised state-space model for the ASP}

The starting point of the process of obtaining an SMLP system for the ASP is to obtain approximations of nonlinear model described in Eq. 48 and Eq. 49. The system was linearised at $n_{m}$ different points and the resulting system was assembled in a state-space format, as shown in Eq. 60 and Eq. 61. The linearised matrices are given in Eq. 62 to Eq. 65.

$$
\boldsymbol{\delta}_{\boldsymbol{k}+1}^{\boldsymbol{m}_{n_{\vartheta}}}=\boldsymbol{A}_{\boldsymbol{m}_{\vartheta}} \boldsymbol{\delta}_{\boldsymbol{k}}^{\boldsymbol{m}_{n_{\vartheta}}}+\boldsymbol{B}_{\boldsymbol{m}_{\vartheta}} \Delta \boldsymbol{u}_{\boldsymbol{k}}+\boldsymbol{D}_{\boldsymbol{m}_{\vartheta}} \Delta \boldsymbol{d}_{\boldsymbol{k}}, \vartheta=1, \ldots, n_{m}
$$

Eq. 60 


$$
\boldsymbol{y}_{\boldsymbol{\vartheta}}^{\boldsymbol{k}}=\boldsymbol{C}_{\boldsymbol{m}_{\boldsymbol{\vartheta}}} \boldsymbol{\delta}_{\boldsymbol{k}}^{\boldsymbol{m}_{\boldsymbol{n}_{\vartheta}}}, \vartheta=1, \ldots, n_{m}
$$

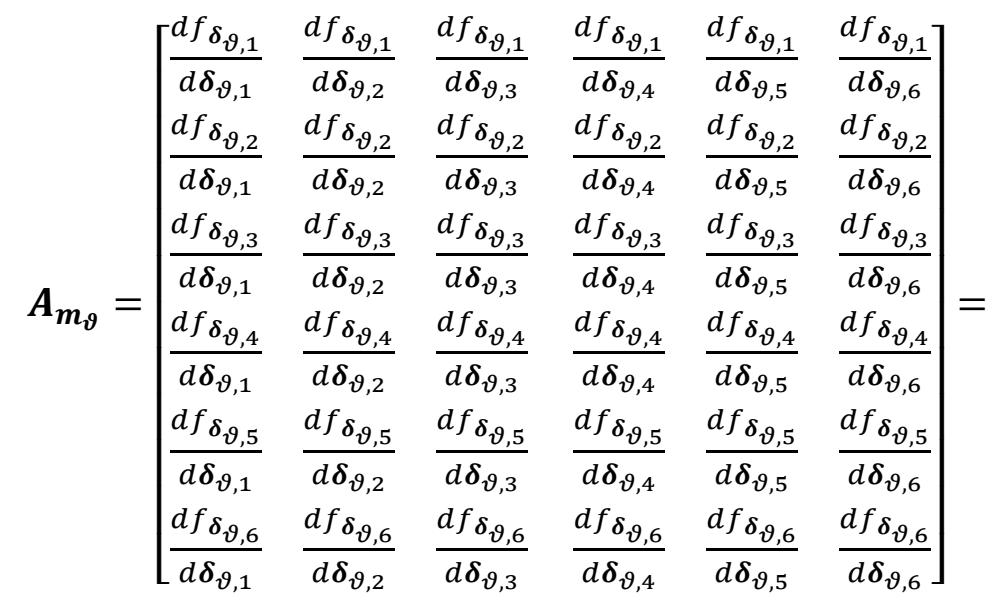

\begin{tabular}{|c|c|c|c|c|c|}
\hline$\left[\begin{array}{c}\frac{\mu_{\max } y_{y_{1}} s_{1}}{\left(K_{s}+s_{1}\right)}-2 K_{d} \frac{x_{1}}{s_{1}} \\
-K_{c}-\frac{\left(q_{i}+q_{r}\right)}{V_{1}}\end{array}\right]$ & {$\left[\begin{array}{c}\mu_{\max }\left(y_{c} x_{1} K_{s}\right. \\
\left(K_{s}+s_{1}\right)^{2} \\
+K_{d}\left(\frac{x_{1}}{\frac{1}{2}}\right)^{2}\end{array}\right]$} & [0] & {$[0]$} & {$[0]$} & {$\left[\frac{q_{r}}{v_{1}}\right]$} \\
\hline$\left[\begin{array}{c}\frac{-\mu_{m a x} s_{1}}{\left(K_{s} s_{1}\right.}+ \\
\frac{2 f_{k d} x_{1} K_{d}}{s_{1}}+f_{k d} K_{c}\end{array}\right]$ & {$\left[\begin{array}{c}\frac{-\mu_{m a x} x_{1} S_{s}}{\left(K_{s} s_{s}\right)^{2}} \\
-f_{k d} K_{d}\left(\frac{x_{1}}{s_{1}}\right)^{2}-\frac{q_{i}}{V_{1}}\end{array}\right]$} & [0] & {$[0]$} & [0] & {$[0]$} \\
\hline$\left[\frac{K_{01} \mu_{\max } s_{1}}{\left(K_{s}+S_{1}\right)}\right]$ & {$\left[\frac{K_{01} \mu_{\max } x_{1} K_{s}}{\left(K_{s}+s_{1}\right)^{2}}\right]$} & {$\left[\begin{array}{l}-K_{l a} f_{k 1} \\
-\frac{\left(q_{i}+q_{r}\right)}{V_{1}}\end{array}\right]$} & {$[0]$} & {$[0]$} & {$[0]$} \\
\hline [0] & [0] & [0] & {$\left[\begin{array}{c}\frac{a_{p}-q_{i}}{A_{s} l_{d}}+ \\
\frac{n r}{l_{d}}\left(\operatorname{arx}_{d} e^{\left(-a r x_{d}\right)}-e^{\left(-\operatorname{ar} x_{d}\right)}\right)\end{array}\right]$} & {$\left[\frac{a_{i}-q_{p}}{A_{s} l_{d}}\right]$} & [0] \\
\hline$\left[\frac{a_{i}+q_{r}}{A_{s} s_{b}}\right]$ & [0] & [0] & {$\left[\frac{n r}{l_{d}}\left(e^{\left(-\operatorname{ar} x_{d}\right)}-\operatorname{arx}_{d} e^{\left(-\operatorname{ar} x_{d}\right)}\right)\right]$} & {$\left[\begin{array}{c}\frac{n r}{l_{b}}\left(a r x_{b} e^{\left(-a r x_{b}\right)}-e^{\left(-a r x_{b}\right)}\right) \\
-\frac{\left(q_{i}+q_{r}\right)}{A_{l} l_{b}}\end{array}\right]$} & {$[0]$} \\
\hline$[0]$ & [0] & [0] & [0] & {$\left[\begin{array}{c}\frac{q_{r}+q_{p}}{A_{s} l_{r}}+ \\
\frac{n r}{l_{r}}\left(e^{\left(-\operatorname{ar} x_{b}\right)}-\operatorname{arx}_{b} e^{\left(-\operatorname{ar} x_{b}\right)}\right)\end{array}\right]$} & {$\left[-\frac{\left(q_{r}+q_{p}\right)}{A_{s} l_{r}}\right]$} \\
\hline
\end{tabular}




$$
\begin{aligned}
& \boldsymbol{B}_{\boldsymbol{m}_{\vartheta}}=\left[\begin{array}{cc}
\frac{d f_{\delta_{\vartheta, 1}}}{d u_{1}} & \frac{d f_{\delta_{\vartheta, 1}}}{d u_{2}} \\
\frac{d f_{\delta_{\vartheta, 2}}}{d u_{1}} & \frac{d f_{\delta_{\vartheta, 2}}}{d u_{2}} \\
\frac{d f_{\delta_{\vartheta, 3}}}{d u_{1}} & \frac{d f_{\delta_{\vartheta, 3}}}{d u_{2}} \\
\frac{d f_{\delta_{\vartheta, 4}}}{d u_{1}} & \frac{d f_{\delta_{\vartheta, 4}}}{d u_{2}} \\
\frac{d f_{\delta_{\vartheta, 5}}}{d u_{1}} & \frac{d f_{\delta_{\vartheta, 5}}}{d u_{2}} \\
\frac{d f_{\delta_{\vartheta, 6}}}{d u_{1}} & \frac{d f_{\delta_{\vartheta, 6}}}{d u_{2}}
\end{array}\right]=\left[\begin{array}{cc}
{\left[\frac{\delta_{6}-\delta_{1}}{V_{1}}\right]} & {[0]} \\
{[0]} & {[0]} \\
{\left[-\frac{\delta_{3}}{V_{1}}\right]} & {[0]} \\
{[0]} & {\left[\frac{\delta_{4}-\delta_{5}}{A_{s} l_{d}}\right]} \\
{\left[\frac{\delta_{1}-\delta_{5}}{A_{s} l_{b}}\right]} & {[0]} \\
{\left[\frac{\delta_{5}-\delta_{6}}{A_{s} l_{r}}\right]} & {\left[\frac{\delta_{5}-\delta_{6}}{A_{s} l_{r}}\right]}
\end{array}\right] \\
& \boldsymbol{C}_{\boldsymbol{m}_{\vartheta}}=\left[\begin{array}{llllll}
0 & 0 & 0 & 0 & 0 & 0 \\
0 & 1 & 0 & 0 & 0 & 0 \\
0 & 0 & 0 & 0 & 0 & 0 \\
0 & 0 & 0 & 0 & 0 & 0 \\
0 & 0 & 0 & 0 & 0 & 0 \\
0 & 0 & 0 & 0 & 0 & 0
\end{array}\right] \\
& \boldsymbol{D}_{\boldsymbol{m}_{\vartheta}}=\left[\begin{array}{ll}
\frac{d f_{\delta_{\vartheta, 1}}}{d d_{1}} & \frac{d f_{\delta_{\vartheta, 1}}}{d d_{2}} \\
\frac{d f_{\delta_{\vartheta, 2}}}{d d_{1}} & \frac{d f_{\delta_{\vartheta, 2}}}{d d_{2}} \\
\frac{d f_{\delta_{\vartheta, 3}}}{d d_{1}} & \frac{d f_{\delta_{\vartheta, 3}}}{d d_{2}} \\
\frac{d f_{\delta_{\vartheta, 4}}}{d d_{1}} & \frac{d f_{\delta_{\vartheta, 4}}}{d d_{2}} \\
\frac{d f_{\delta_{\vartheta, 5}}}{d d_{1}} & \frac{d f_{\delta_{\vartheta, 5}}}{d d_{2}} \\
\frac{d f_{\delta_{\vartheta, 6}}}{d d_{1}} & \frac{d f_{\delta_{\vartheta, 6}}}{d d_{2}}
\end{array}\right]=\left[\begin{array}{cc}
{[0]} & {\left[\frac{x_{i}-\delta_{1}}{V_{1}}\right]} \\
{\left[\frac{d_{2}}{V_{1}}\right]} & {\left[\frac{d_{1}-\delta_{2}}{V_{1}}\right]} \\
{[0]} & {\left[-\frac{\delta_{3}}{V_{1}}\right]} \\
{[0]} & {\left[\frac{\delta_{5}-\delta_{4}}{A_{s} l_{d}}\right]} \\
{[0]} & {\left[\frac{\delta_{1}-\delta_{5}}{A_{s} l_{b}}\right]} \\
{[0]} & {[0]}
\end{array}\right]
\end{aligned}
$$

Eq. 64

Eq. 65

\subsubsection{PWA and SMLP representations of the ASP}

The operating point (OP), the identification points (IPs) and the partitions for the PWA system will be defined, respectively, as the input vectors (MVs + DVs) and input ranges. Table 5 provides the IPs values used to obtain the models:

Table 5 - Input-based IPs identification points 1,2 and 3 .

\begin{tabular}{|l|c|c|c|c|}
\hline & $u_{\mathbf{1}}\left(\boldsymbol{m}^{\mathbf{3}} / \boldsymbol{h}\right)$ & $\boldsymbol{u}_{\mathbf{2}}\left(\boldsymbol{m}^{\mathbf{3}} / \boldsymbol{h}\right)$ & $\boldsymbol{d}_{\mathbf{1}}(\boldsymbol{m g} / \boldsymbol{L})$ & $\boldsymbol{d}_{\mathbf{2}}\left(\boldsymbol{m}^{\mathbf{3}} / \boldsymbol{h}\right)$ \\
\hline $\boldsymbol{I P}_{\mathbf{1}}$ & 170 & 1020 & 1 & 1700 \\
\hline $\boldsymbol{I} \boldsymbol{P}_{\mathbf{2}}$ & 720 & 45 & 0.70 & 900 \\
\hline $\boldsymbol{I} \boldsymbol{P}_{\mathbf{3}}$ & 400 & 400 & 4.100 & 1000 \\
\hline
\end{tabular}


The PWA partition rules i.e., the boundaries of the active region of each linearisation, were defined by the arithmetic mean between the model's linearisation states. Due to the multivariable nature of this control problem, a conflict may arise when selecting the active model (the one used to update the state): a certain OP might lie inside the validity region of different models at the same time if multiple variables are considered. Therefore, the PWA model selection will be given by a voting system: each variable casts a vote according to which space partition it is currently within, and the model with more votes is going to be selected. In the case of a tie, the current model has the preference if it is one the models with the same number of votes; otherwise a random selection of the new active model occurs. The PWA partition definitions and voting rules are provided below in Eq. 66 to Eq. 69. Variables $M_{1}^{\text {vote }}, M_{2}^{\text {vote }}$ and $M_{3}^{\text {vote }}$ signify the number of votes cast to each model according to the position of each variable. They are set to zero each time the prediction is computed.

$$
\begin{aligned}
& \text { if } u_{1} \leq 285 \rightarrow M_{1}^{\text {vote }}=M_{1}^{\text {vote }}+1 ; \\
& \text { if } 285<u_{1} \leq 560 \rightarrow M_{3}^{\text {vote }}=M_{3}^{\text {vote }}+1 ; \\
& \text { if } 560<u_{1} \rightarrow M_{2}^{\text {vote }}=M_{2}^{\text {vote }}+1 ; \\
& \text { if } u_{2} \leq 222.5 \rightarrow M_{2}^{\text {vote }}=M_{2}^{\text {vote }}+1 ; \\
& \text { if } 222.5<u_{2} \leq 710 \rightarrow M_{3}^{\text {vote }}=M_{3}^{\text {vote }}+1 ; \\
& \text { if } 710<u_{2} \rightarrow M_{1}^{\text {vote }}=M_{1}^{\text {vote }}+1 ; \\
& \text { if } d_{1} \leq 0.85 \rightarrow M_{2}^{\text {vote }}=M_{2}^{\text {vote }}+1 ; \\
& \text { if } 0.85<d_{1} \leq 2.55 \rightarrow M_{1}^{\text {vote }}=M_{1}^{\text {vote }}+1 ; \\
& \text { if } 2.55<d_{1} \rightarrow M_{3}^{\text {vote }}=M_{3}^{\text {vote }}+1 ; \\
& \text { if } d_{2} \leq 950 \rightarrow M_{2}^{\text {vote }}=M_{2}^{\text {vote }}+1 ; \\
& \text { if } 950<d_{2} \leq 1350 \rightarrow M_{3}^{\text {vote }}=M_{3}^{\text {vote }}+1 ; \\
& \text { if } 1350<d_{2} \rightarrow M_{1}^{\text {vote }}=M_{1}^{\text {vote }}+1 ;
\end{aligned}
$$

As for the SMLP system, SMLP method 2 was chosen to represent the plant, and thus the same IPs defined in Table 5 can be used. A second order polynomial was used 
as an attraction function, and regression analysis was performed to obtain its parameters, thus minimising the error between the nonlinear model and the SMLP system.

\subsubsection{Results comparison}

The linearised models of plants contained in must be BIBO stable and thus not have eigenvalues out of the unit circle. A plant was created which is BIBO stable which is similar to one of the plants presented in Francisco el al. (2011) but with different heights for the first, second and third layers of the settlers (and $\mathrm{l}_{\mathrm{d}}=6 \mathrm{~m}, \mathrm{l}_{\mathrm{b}}=3 \mathrm{~m}, \mathrm{l}_{\mathrm{r}}=4.5 \mathrm{~m}$ ).

The prediction of the single CV of the ASP, the chemical oxygen demand (COD), was obtained from the nonlinear model (Eq. 56 to Eq. 59) as well as from PWA and the SMLP systems. An initial OP distinct from the three linearisation points was selected $\left(\boldsymbol{O P} \boldsymbol{P}_{\boldsymbol{k}}=[345,115,0.40,1150]\right)$, and also a rule is provided for describing the OP as a function of time, which is presented in Eq. 70, yielding a series of control moves and disturbances:

$$
\boldsymbol{O P _ { k + \alpha }}=\left[\begin{array}{c}
O P_{1, k} \cdot \exp (-0.002 \alpha) \\
O P_{2, k} \cdot \exp (-0.002 \alpha)^{-1} \\
O P_{3, k}+10 \cdot[1-\exp (-0.1 \alpha)] \\
O P_{4, k} \cdot(1+\sin (\alpha) / 10)
\end{array}\right], \alpha=1, \ldots, p
$$

Comparing side-by-side the predictions shown in Fig. 9, the SMLP prediction was considerably more accurate and further reduced the error arising from process nonlinearity. One can also see the significant discontinuities caused by sudden changes in the state update rule for the PWA system, and the SMLP approximation is more accurate most of the time. Using the nonlinear model as a reference, the integration of error over the period of 100 minutes yielded $138.32 \mathrm{mg} \cdot \mathrm{min} / \mathrm{L}$ and $76.27 \mathrm{mg} \cdot \mathrm{min} / \mathrm{L}$ for the PWA and the SMLP systems respectively. This translates into a $44.86 \%$ error additional reduction provided by the SMLP method over the PWA in this use scenario. In comparison to developing a rigorous first-principles or hybrid model, the SMLP has the advantage of reducing both computational time (for solving optimisation) and engineering man-hours. 


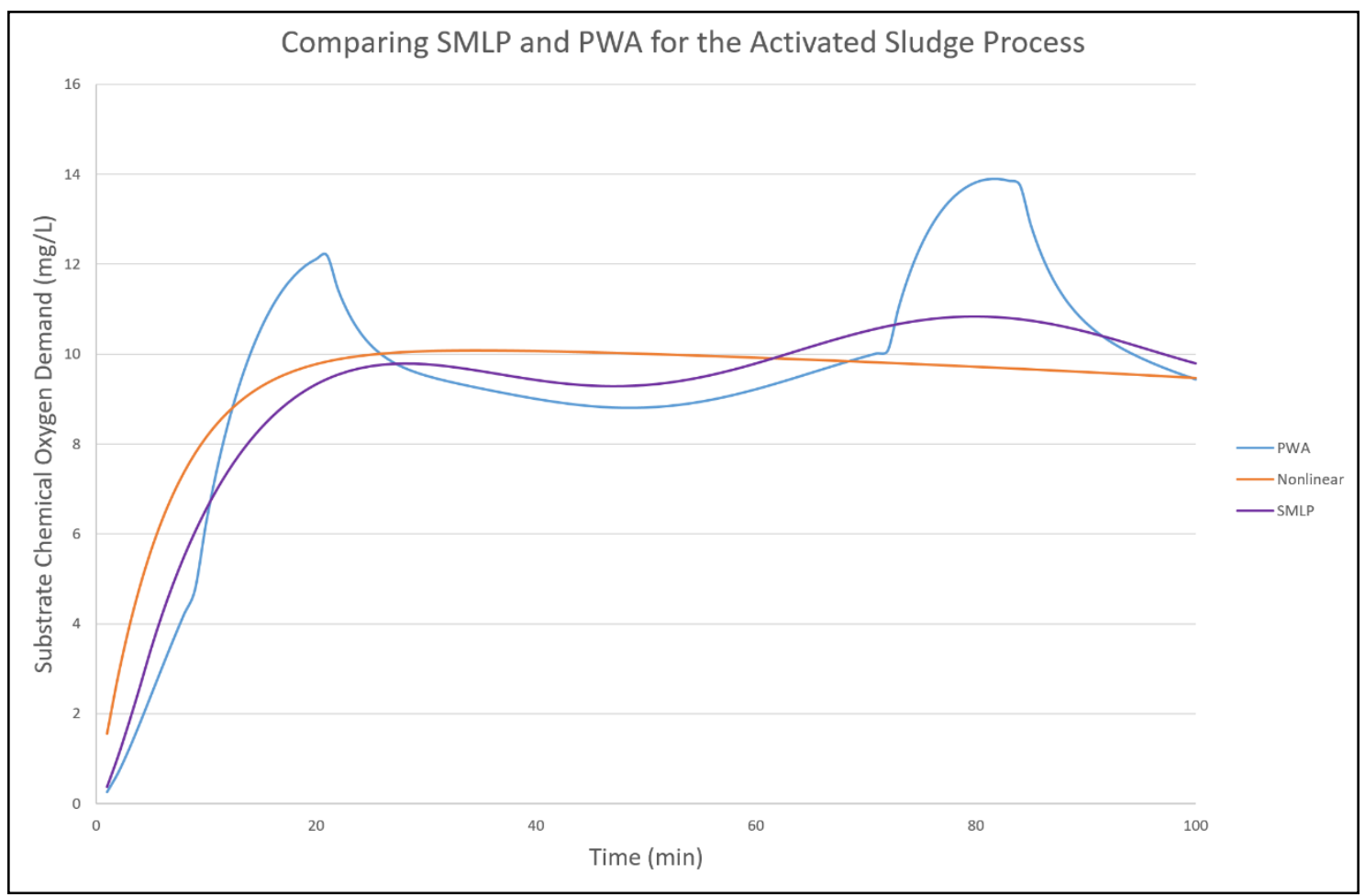

Fig. 9 - Predictions generated by the SMLP and PWA systems and by the ASP nonlinear model.

\section{An updated Economic MPC Optimisation (EMOP) index}

Using optimisation algorithms to find the global minimum of Eq. 71 provides as a solution the optimal OP for each plant design. The reader is encouraged to check Strutzel and Bogle (2016) for further details.

$$
I_{E M O P}=\min _{\Delta \boldsymbol{u}_{\boldsymbol{K}} \in \boldsymbol{U}}\left(\left|\boldsymbol{S} \boldsymbol{L}_{\mathbf{1}}\right|^{-1}\left|\boldsymbol{S} \boldsymbol{L}_{\mathbf{2}}\right|^{-1}\left[J_{k+p}+\left|\boldsymbol{E} \boldsymbol{P}^{-\mathbf{1}}\right|\right]\right)
$$

Where:

$$
\begin{aligned}
& J_{k+p}= \\
& \sum_{i=1}^{n_{y}}+\sum_{j=1}^{n u}\left[\left|y_{i, \min }-y_{k+p, i}\right| \cdot W_{i, \text { lower }}+\left|y_{k+p, i-} y_{i, \max }\right| \cdot W_{i, \text { upper }}\left|\cdot V_{j, \min }+\right| u_{j, \max }-u_{k+p, j} \mid \cdot V_{j, \max }\right]
\end{aligned}
$$

where: $\mathrm{u}_{\mathrm{k}+\mathrm{p}, \mathrm{j}}=\sum_{\mathrm{j}=1}^{\mathrm{m}} \Delta \mathrm{u}_{\mathrm{k}+\mathrm{j}} ; \mathrm{p}$ is the prediction horizon; $\mathrm{V}_{\mathrm{j}, \min }$ and $\mathrm{V}_{\mathrm{j}, \max }$, where $j=1, \ldots, n_{u}$, where $n_{u}$ is the number of MVs, are two sets of optimisation weights which illustrate the optimisation direction and relative priority among the various MVs for economic purposes, defining for each MV whether it is positively or negatively related to profitability, and to what degree; $\mathrm{W}_{\mathrm{i} \text {,upper }}$ and $\mathrm{W}_{\mathrm{i} \text {,lower }}$ are the control zone weights in the 
cost function used to penalise control zone violations, whose values are also provided by process economics; $\mathrm{SL}_{1}$ and $\mathrm{SL}_{2}$ are the "soft landing" matrices, which are inversely proportional respectively to the first and to the second order derivatives of the CVs at instant $\mathrm{k}+\mathrm{p}$, ensuring that the transition to the final state is as smooth as possible; EP is "error penalization" matrix that penalizes temporary violations of the control bounds that may occur during the trajectory between the initial state and the optimal final state. The optimisation problem of Eq. 71 is subject to the following restrictions:

$$
\begin{aligned}
& \text { if } y_{i, \min } \leq y_{k+p, i} \leq y_{i, \max } \Rightarrow W_{i, \text { upper }}=W_{i, \text { lower }}=0 \\
& \text { if } y_{i, \min }>y_{k+p, i} \Rightarrow W_{i, \text { lower }}>0 \\
& \text { if } y_{k+p, i}>y_{i, \max } \Rightarrow W_{i, \text { upper }}>0 \\
& u_{\text {min }} \leq u_{k+p, j} \leq u_{\max } \\
& V_{j, \min } \geq 0, V_{j, \max } \geq 0, V_{j, \min } \cdot V_{j, \max }=0
\end{aligned}
$$

For the updated case study of Section 6, it is necessary to make changes in the original form of the EMOP index to include price variations in the cost function. The possibility of producing premium as opposed to regular priced products can be incorporated into the EMOP index's cost function by adding a new term for each product price variation. This new term is a function of a new variable defined as the difference between the key CV quality threshold value for which the price variation occurs, $\bar{y}_{t}$, and the average value of the key $\mathrm{CV}$ through the prediction horizon, $\overline{\mathrm{y}}_{\text {ave }}$, as shown in Eq. 78 and Eq. 79:

$$
\begin{aligned}
& \text { if }\left(\bar{y}_{\text {qual }}-\bar{y}_{\text {ave }}\right) \geq 0, \Delta \bar{y}_{\text {qual }}=\left(\bar{y}_{\text {qual }}-\bar{y}_{\text {ave }}\right) \\
& \text { if }\left(\bar{y}_{\text {qual }}-\bar{y}_{\text {ave }}\right)<0, \Delta \bar{y}_{\text {qual }}=0
\end{aligned}
$$

So the added cost function value, $\mathrm{j}_{\mathrm{q}}$, is defined in Eq. 80:

$$
j_{q}=\Delta \bar{y}_{q u a l} \cdot \frac{\Delta \$_{\text {qual }}}{v_{\text {prod }}} \cdot v_{\text {prod }}
$$

Where $\Delta \$_{\text {qual }}$ is the added value, i.e., product price difference between premium priced and regular product, and $v_{\text {prod }}$ is the volume produced of the said product. If there 
is more than one quality threshold for a single $\mathrm{CV}$, adding the price increments of all the consecutive thresholds to the price of the regular product, $\$_{\text {reg }}$, should yield the price of the premium variant, $\$_{\text {qual, }} \mathrm{n}_{\mathrm{q}}$, as shown in Eq. 81:

$$
\begin{aligned}
& \$_{q u a l, 1}=\$_{r e g}+\Delta \$_{q u a l, 1} \\
& \vdots \\
& \$_{q u a l, n q}=\$_{r e g}+\sum_{j=1}^{n_{q}} \Delta \$_{q u a l, j}
\end{aligned}
$$

where $\mathrm{n}_{\mathrm{q}}$ is the number of quality thresholds of the product being considered. For processes with several CVs with quality thresholds, it is useful to aggregate all added cost function values in a single term, as per Eq. 82:

$$
J_{q}=\sum_{i=1}^{n_{y}} \sum_{j=1}^{n_{q}, i} \Delta \bar{y}_{q u a l, i} \cdot \frac{\Delta \$_{q u a l, i, j}}{v_{\text {prod }, 1}} \cdot v_{\text {prod }, i}
$$

where $\mathrm{J}_{\mathrm{q}}$ is total opportunity cost due to quality thresholds. This way, $\mathrm{J}_{\mathrm{q}}$ becomes null if only the highest premium priced product variants are being produced. Adding Eq. 82 to the standard EMOP index, Eq. 71, yields the EMOP index with quality thresholds:

$$
I_{E M O P}=\min _{\Delta \boldsymbol{u}_{\boldsymbol{K}} \in \boldsymbol{U}}\left(\left|\boldsymbol{S} \boldsymbol{L}_{\mathbf{1}}\right|^{-1}\left|\boldsymbol{S} \boldsymbol{L}_{\mathbf{2}}\right|^{-1}\left[J_{k+p}+J_{q}+\left|\boldsymbol{E} \boldsymbol{P}^{-1}\right|\right]\right)
$$

This index definition of Eq. 83 is appropriate to assess processes with variable product prices such as our crude oil distillation case study.

\section{Case of Study - EMOP Index for an Oil Distillation Process Unit}

In order to demonstrate how the SMLP approach can be useful in providing accurate predictions for the dynamic behaviour of a complex flowsheet, we returned to the crude oil distillation case study presented in the earlier paper, Strutzel and Bogle (2016). The same four designs are represented as SMLP systems and evaluated again using the EMOP index methodology. Plant 1 is the base case flowsheet, using the standard layout shown in Fig. 10. Plant 2 is identical to plant 1 except it has the stream analysers placed downstream of product tanks which collect, respectively, kerosene and pool diesel output streams. The analysers, which measures product properties are located at the output of these tanks for plant 2, and at the inlet for the other plants. Plant 3 and 4 are also 
very similar to plant 1 , but with distillation columns of significantly different dimensions. Plant 3's column is of increased size compared to plants 1 and 2's, while Plant 4's has a smaller column accompanied by a pre-flash drum that removes the lighter fractions such as $\mathrm{C} 1-\mathrm{C} 4$ gases and light naphtha and an extra fired heater.

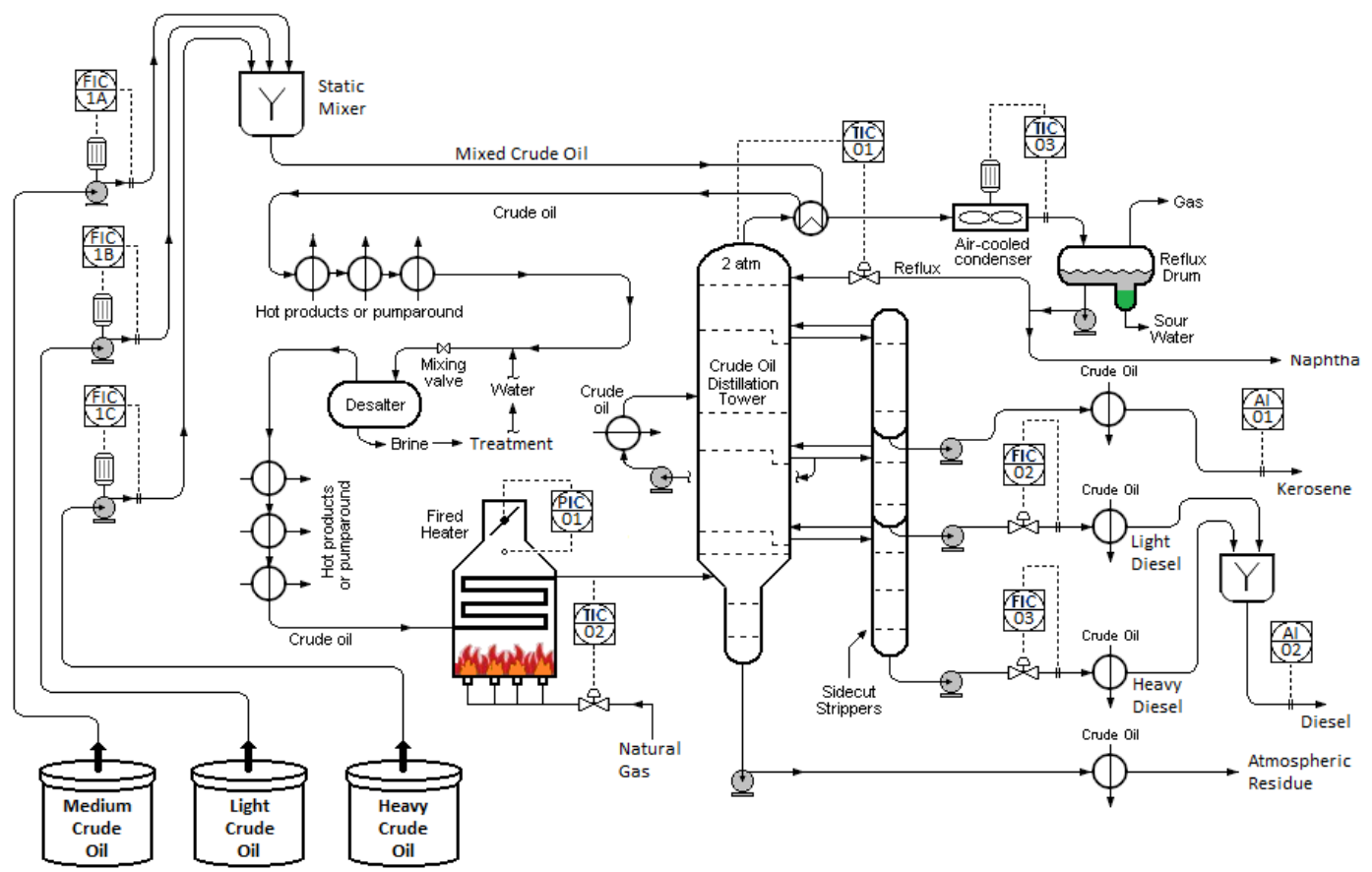

Fig. 10 - Plant 1 - Simplified Process Flowsheet.

A change from the earlier paper is the introduction of price variations in the EMOP cost function according to the quality of diesel being produced. 
Table 6 provides example costs for the crude oils and the prices for the products, which will be used in the simulation to calculate the profitability of the process. Now the diesel oil has different prices depending on its Cetane Index (or CI) since a premium is charged for high-performance fuel. Higher CI fuels provided benefits such as quicker starting, quieter operation, and improved fuel efficiency, among others. This was included to make the case study more realistic since most refineries operate with variable grades of product quality and prices. 
Table 6 - Crude Oil Costs and Product Prices.

\begin{tabular}{|c|c|c|}
\hline Stream & $\mathrm{US} \$ / \mathrm{m}^{3}$ & Price \\
\hline Medium Crude Oil & $\mathrm{US} \$ / \mathrm{m}^{3}$ & 430.06 \\
\hline Heavy Crude Oil & $\mathrm{US} \$ / \mathrm{m}^{3}$ & 398.21 \\
\hline Light Crude Oil & $\mathrm{US} \$ / \mathrm{m}^{3}$ & 471.92 \\
\hline Naphtha & $\mathrm{US} \$ / \mathrm{m}^{3}$ & 484.84 \\
\hline Kerosene & $\mathrm{US} \$ / \mathrm{m}^{3}$ & 557.83 \\
\hline Diesel - CI 46 & $\mathrm{US} \$ / \mathrm{m}^{3}$ & 551.21 \\
\hline Diesel - CI 48 & $\mathrm{US} \$ / \mathrm{m}^{3}$ & 564.48 \\
\hline Diesel - CI 50 & $\mathrm{US} \$ / \mathrm{m}^{3}$ & 583.88 \\
\hline Atmospheric Residue & $\mathrm{US} \$ / \mathrm{m}^{3}$ & 446.66 \\
\hline
\end{tabular}

The minimum diesel production was set to $140 \mathrm{~m}^{3} / \mathrm{h}$. All other details that defined the optimisation problem remained the same as in Strutzel and Bogle (2016), including the vectors $\mathrm{W}_{\text {lower }}, \mathrm{W}_{\text {upper }}, \mathrm{V}_{\min }$ and $\mathrm{V}_{\mathrm{max}}$, which were obtained from an analysis of process economics.

\subsection{Applying the SMLP approach for the Crude Oil distillation}

In this paper, single linear state-space models no longer provided the output predictions for the four crude oil distillation plants, as it has been done in Strutzel and Bogle (2016). This time the plants were modelled as SMLP systems, each one composed of three sub-models.

The first step to build an SMLP system is defining the IPs (identification points) for each plant. Each IP may be defined as an output (CV) set for use with SMLP method 1 or, alternatively, as an input (MV+DV) set for use with SMLP methods 2 and 3. Table 7 provides the IPs as input sets, which are common for all the four plant designs:

Table 7 - Input-based IPs identification points 1, 2 and 3.

\begin{tabular}{|l|c|c|c|c|c|c|c|c|}
\hline & $\boldsymbol{u}_{\mathbf{1}}(\boldsymbol{C})$ & $\boldsymbol{u}_{\mathbf{2}}(\boldsymbol{C})$ & $\boldsymbol{u}_{\mathbf{3}}\left(\boldsymbol{m}^{\mathbf{3}} / \boldsymbol{h}\right)$ & $\boldsymbol{u}_{\mathbf{4}}\left(\boldsymbol{m}^{\mathbf{3}} / \boldsymbol{h}\right)$ & $\boldsymbol{u}_{\mathbf{5}}\left(\boldsymbol{m}^{\mathbf{3}} / \boldsymbol{h}\right)$ & $\boldsymbol{u}_{\mathbf{6}}\left(\boldsymbol{m}^{\mathbf{3}} / \boldsymbol{h}\right)$ & $\begin{array}{l}\boldsymbol{u}_{\mathbf{7}}\left(\boldsymbol{m}^{\mathbf{3}}\right. \\
/ \boldsymbol{h})\end{array}$ & $\begin{array}{l}\boldsymbol{d}_{\mathbf{1}}\left(\boldsymbol{m}^{\mathbf{3}}\right. \\
/ \boldsymbol{h})\end{array}$ \\
\hline $\boldsymbol{I} \boldsymbol{P}_{\mathbf{1}}$ & 44.4 & 341.1 & 139.5 & 29.7 & 550.0 & 230.0 & 30.0 & 5.0 \\
\hline $\boldsymbol{I} \boldsymbol{P}_{\mathbf{2}}$ & 47.4 & 342.8 & 134.0 & 29.9 & 470.0 & 210.0 & 110.0 & 25.0 \\
\hline $\boldsymbol{I P}_{\mathbf{3}}$ & 50.0 & 344.5 & 128.5 & 40.0 & 400.0 & 200.0 & 170.0 & 45.0 \\
\hline
\end{tabular}

The magnitudes of the IPs were determined arbitrarily with a view to covering a wide range of possible operating points, i.e., distinct crude oil feed compositions obtained from different mixes of light, medium and heavy oil. Basically, $\boldsymbol{I} \boldsymbol{P}_{\mathbf{1}}$ is a light oil mix, $\boldsymbol{I} \boldsymbol{P}_{\mathbf{2}}$ is a standard oil mix, and $\boldsymbol{I} \boldsymbol{P}_{3}$ is a heavy oil mix. A heavier oil mix is cheaper to acquire but harder to refine into on-spec products. One could interpret the steady state $\boldsymbol{I P}_{3}$ as representing the cheapest oil mix that can still successfully produce marketable products. On the other hand, $\boldsymbol{I} \boldsymbol{P}_{\mathbf{1}}$ is a premium mix that can provide a much higher yield of high 
added-value products. $\boldsymbol{I} \boldsymbol{P}_{2}$ represents a typical situation between the cheapest and the premium oil mix.

Since the designs are all different, note that for each flowsheet the same inputs will result in a different output, i.e., the output-based IPs are not equal between plants, even if the input-based IPs are. The resulting output vectors for each plant are presented in Table 8, and are thus used as IPs for SMLP method 1:

Table 8 - Output-based IPs Identification points 1, 2 and 3.

\begin{tabular}{|c|c|c|c|c|c|c|c|c|c|c|c|c|}
\hline & \multicolumn{3}{|c}{ plant 1 } & \multicolumn{3}{c|}{ plant $\mathbf{2}$} & \multicolumn{3}{c|}{ plant 3 } & \multicolumn{3}{c|}{ plant 4 } \\
\hline & $\mathbf{I P}_{\mathbf{1}}$ & $\mathbf{I P}_{\mathbf{2}}$ & $\mathbf{I P}_{\mathbf{3}}$ & $\mathbf{I P}_{\mathbf{1}}$ & $\mathbf{I P}_{\mathbf{2}}$ & $\mathbf{I P}_{\mathbf{3}}$ & $\mathbf{I P}_{\mathbf{1}}$ & $\mathbf{I P}_{\mathbf{2}}$ & $\mathbf{I P}_{\mathbf{3}}$ & $\mathbf{I P}_{\mathbf{1}}$ & $\mathbf{I P}_{\mathbf{2}}$ & $\mathbf{I P}_{\mathbf{3}}$ \\
\hline $\mathbf{y}_{\mathbf{1}}$ & 41.05 & 41.53 & 42.58 & 39.67 & 40.18 & 40.87 & 41.05 & 41.54 & 42.58 & 41.22 & 41.78 & 42.82 \\
\hline $\mathbf{y}_{\mathbf{2}}$ & 83.29 & 86.11 & 89.05 & 74.07 & 76.88 & 81.14 & 83.29 & 86.10 & 89.06 & 72.78 & 78.24 & 84.01 \\
\hline $\mathbf{y}_{\mathbf{3}}$ & 290.98 & 298.14 & 308.48 & 290.39 & 298.46 & 312.94 & 290.95 & 298.24 & 308.45 & 298.30 & 308.66 & 322.38 \\
\hline $\mathbf{y}_{\mathbf{4}}$ & 322.45 & 329.96 & 344.25 & 321.67 & 330.47 & 350.64 & 322.40 & 330.17 & 344.20 & 344.25 & 357.84 & 379.75 \\
\hline $\mathbf{y}_{\mathbf{5}}$ & 344.69 & 354.15 & 371.11 & 343.90 & 354.77 & 378.55 & 344.63 & 354.44 & 371.03 & 379.72 & 398.03 & 436.43 \\
\hline $\mathbf{y}_{\mathbf{6}}$ & -24.61 & -21.70 & -17.56 & -26.82 & -23.63 & -19.11 & -24.62 & -21.67 & -17.57 & -24.14 & -20.18 & -15.34 \\
\hline $\mathbf{y}_{\mathbf{7}}$ & 826.45 & 830.10 & 834.63 & 826.39 & 830.09 & 835.42 & 826.46 & 830.08 & 834.64 & 832.02 & 836.69 & 842.62 \\
\hline $\mathbf{y}_{\mathbf{8}}$ & 250.78 & 256.66 & 265.25 & 250.40 & 256.67 & 269.52 & 250.74 & 256.69 & 265.20 & 219.36 & 235.00 & 239.67 \\
\hline $\mathbf{y}_{\mathbf{9}}$ & 50.13 & 54.38 & 62.03 & 49.93 & 54.58 & 63.11 & 50.12 & 54.44 & 62.06 & 9.71 & 16.34 & 23.64 \\
\hline $\mathbf{y}_{\mathbf{1 0}}$ & 813.16 & 816.71 & 819.61 & 813.05 & 816.72 & 820.49 & 813.15 & 816.72 & 819.62 & 791.55 & 798.52 & 802.88 \\
\hline $\mathbf{y}_{\mathbf{1 1}}$ & -68.32 & -64.32 & -58.76 & -68.45 & -64.15 & -56.72 & -68.33 & -64.30 & -58.77 & -76.77 & -75.03 & -73.62 \\
\hline
\end{tabular}

Now that IPs are fully defined for all plants, it is necessary to generate reference trajectories for the regression analysis. When generating an SMLP system, it is recommended that one performs a comprehensive regression analysis to obtain adequate constants for the degradation functions. As per Eq. 18 and Eq. 30, model error related to the nonlinear dynamic simulator must be minimised by testing several trajectories in order to identify the optimal constants. The three SMLP methods detailed in Section 3 were tested and the results are provided in this Section. To generate the regression analysis problems for each plant, a total of six distinct reference trajectories were used, i.e., three trajectories as a training dataset, and three as a validation dataset. These trajectories were generated manipulating the plant inputs in the dynamic simulator as defined by Eq. 84 to Eq. 89, through a prediction horizon of $k=1, \ldots, 10$ (time unit is 10 minutes). 


$$
\begin{aligned}
& \boldsymbol{O P} \boldsymbol{P}_{1, \boldsymbol{k}}^{\text {Set1 }}=I \boldsymbol{P}_{1}+[1.5 ;-3 ; 0 ; 0 ; 40 ;-40 ; 0 ; 0]\left[\cos \left(\frac{k}{6.5}\right)+2 \sin ^{4}\left(\frac{k}{11}\right)\right]+ \\
& {[0 ; 0 ; 9 ;-2.2 ; 0 ; 0 ; 8.8 ;-1.4]\left[3.3 \sin ^{2}(2 k)+1,88 \cos ^{2}\left(\frac{k}{6.2}\right)-2\right]} \\
& \boldsymbol{O} \boldsymbol{P}_{2, \boldsymbol{k}}^{\text {Set1 }}=I \boldsymbol{P}_{2}+[0 ; 3 ; 0 ; 2.1 ; 0 ; 40 ; 0 ; 2]\left[\sin ^{3}\left(\frac{k}{5.9}\right)+\cos \left(\frac{k}{7.4}\right)\right] \\
& +[-1.6 ; 0 ;-14 ; 0 ;-43 ; 0 ;-43 ; 0]\left[\sin ^{4}\left(\frac{k}{2.8}\right)+\sin ^{6}\left(\frac{k}{3.8}\right)\right] \\
& \boldsymbol{O} P_{3, \boldsymbol{k}}^{\text {Set1 }}=\boldsymbol{I} \boldsymbol{P}_{3}+[0.25 ;-0.46 ; 2.05 ;-0.5 ; 0 ; 0 ; 0 ; 0]\left(\frac{k}{9}\right) \sin \left(\frac{k}{5.3}\right)+ \\
& {[0 ; 0 ; 0 ; 0 ; 33.21 ;-33.21 ; 33.21 ;-1.7] 8.8 k^{-0.65} \sin \left(\frac{k}{13}\right) \cos \left(\frac{k}{7}\right)}
\end{aligned}
$$

$$
\begin{aligned}
& \boldsymbol{O} \boldsymbol{P}_{1, \boldsymbol{k}}^{\text {Set2 }}=\boldsymbol{I} \boldsymbol{P}_{1}+[0.45 ; 0 ; 4.34 ; 0 ; 15 ; 0 ; 4 ; 0] \frac{k}{10} \cos \left(\frac{k}{5}\right)+ \\
& {[0 ;-1.9 ; 0 ;-2.9 ; 0 ;-35 ; 0 ;-1] \frac{10}{k^{0.5}} \sin \left(\frac{k}{10}\right) * \cos \left(\frac{k}{10}\right)} \\
& \boldsymbol{O P}_{2, \boldsymbol{k}}^{\text {Set2 }}=I \boldsymbol{P}_{2}+[-1.13 ; 2.8 ;-13 ; 3.1 ; 0 ; 0 ; 0 ; 0]\left[\sin ^{3}\left(\frac{k}{11.5}\right)+\right. \\
& \left.\cos ^{3}\left(\frac{k}{3.75}\right)\right]+[0 ; 0 ; 0 ; 0 ;-45 ; 37.5 ;-30 ; 2.6]\left[\sin ^{2}\left(\frac{k}{2}\right)+\cos ^{4}\left(\frac{k}{4}\right)\right] \\
& \boldsymbol{O P}_{3, \boldsymbol{k}}^{\text {Set2 }}=\boldsymbol{I} \boldsymbol{P}_{3}+[2 ;-1.850 ; 0 ; 22 ;-17 ; 0 ; 0]\left[\sin \left(\frac{k}{8,5}\right)+2 \cos ^{3}\left(\frac{k}{6}\right) \wedge 3\right] \\
& +[0 ; 0 ; 1.55 ;-0.41 ; 0 ; 0 ; 5.6 ;-0.43]\left[4 \sin ^{2}\left(\frac{k}{3}\right)+3 \sin ^{4}\left(\frac{k}{5}\right)\right]
\end{aligned}
$$

where $\mathrm{IP}_{1}, \mathrm{IP}_{2}$ and $\mathrm{IP}_{3}$ are each sub-models' input-based IPs (Table 8). Now we are going to present the results from regression analysis problems presented in Sections 3.2, 3.3 and 3.4. The goal was to assess which of the three method variants had better performance and if the difference was significant. The analysis consists of minimising the sum of the normalised squared prediction error (due to linearisation) for all variables through the prediction horizon $(\mathrm{k}=1, \ldots, \mathrm{p})$, for each one of the 3 reference trajectories (training dataset), as defined by Eq. 18 (methods 1 and 2) and Eq. 30 (method 3). Also in the first three rows of Table 9 are the prediction error values for the linear models identified at $\mathrm{IP}_{1}, \mathrm{IP}_{2}$ and $\mathrm{IP}_{3}$, which were calculated using Eq. 19. One can notice the values very widely, and some linear models are more robust than others, meaning they are much better at predicting trajectories far removed from the surroundings of their IPs.

Table 9 contains the optimised objective function values for plants 1, 2, 3 and 4 using the 3 SMLP methods. 
At first, the regression analysis was performed using $2^{\text {nd }}$ order degradation functions (keeping $g_{\vartheta, 4}=g_{\vartheta, 3}=0$, and allowing $g_{\vartheta, 2}, g_{\vartheta, 1}$ and $g_{\vartheta, 0}$ to assume any real value in the interval $0<g \leq 10^{7}$ ). Then another regression analysis was carried out, using the solution the $2^{\text {nd }}$ order degradation as an initial guess but allowing $g_{\vartheta, 3}$ to vary. Finally, the solution of the $3^{\text {rd }}$ order degradation is used as an initial guess of the $4^{\text {th }}$ order polynomial, but this time $g_{\vartheta, 4}$ also varies. The aim of this procedure was to assess if additional parameters would further reduce the model mismatch, as compared to using a $2^{\text {nd }}$ order polynomial, or if they would cause overfitting and increase overall error.

Also in the first three rows of Table 9 are the prediction error values for the linear models identified at $\mathrm{IP}_{1}, \mathrm{IP}_{2}$ and $\mathrm{IP}_{3}$, which were calculated using Eq. 19. One can notice the values very widely, and some linear models are more robust than others, meaning they are much better at predicting trajectories far removed from the surroundings of their IPs.

Table 9 contains the sum of numeric residue (normalised squared error) for SMLP methods 1,2 and 3 as compared to the training dataset, through the prediction horizon. Also in the first three rows of Table 9 are the prediction error values for the linear models identified at $\mathrm{IP}_{1}, \mathrm{IP}_{2}$ and $\mathrm{IP}_{3}$, which were calculated using Eq. 19. One can notice the values very widely, and some linear models are more robust than others, meaning they are much better at predicting trajectories far removed from the surroundings of their IPs.

Table 9 - Model mismatch with relation to the validation dataset (Set 1).

\begin{tabular}{|c|c|c|c|c|}
\hline Total normalised squared error & Plant 1 & Plant 2 & Plant 3 & Plant 4 \\
\hline Linear sub-model $1\left(\right.$ IP $\left._{\mathbf{1}}\right)$ & 314.903 & 49.492 & 418.844 & 266.311 \\
\hline Linear sub-model $2\left(I_{2}\right)$ & 461.080 & 47.683 & 310.134 & 1207.434 \\
\hline Linear sub-model $3\left(\mathrm{IP}_{\mathbf{3}}\right)$ & 614.280 & 25.612 & 627.542 & 165.818 \\
\hline SMLP Method $1-2^{\text {nd }}$ order & 293.038 & 24.392 & 286.384 & 74.664 \\
\hline SMLP Method $1-3^{\text {rd }}$ order & 292.989 & 23.919 & 285.662 & 74.365 \\
\hline SMLP Method $1-4^{\text {th }}$ order & 292.894 & 23.925 & 285.601 & 73.694 \\
\hline SMLP Method $2-2^{\text {nd }}$ order & 291.591 & 19.929 & 291.334 & 87.891 \\
\hline SMLP Method $2-3^{\text {rd }}$ order & 291.328 & 20.616 & 291.280 & 86.650 \\
\hline SMLP Method $2-4^{\text {th }}$ order & 289.631 & 21.511 & 291.279 & 84.942 \\
\hline SMLP Method $3-2^{\text {nd }}$ order & 253.351 & 19.606 & 220.956 & 92.356 \\
\hline SMLP Method $3-3^{\text {rd }}$ order & 250.775 & 18.837 & 214.433 & 90.091 \\
\hline SMLP Method $3-4^{\text {th }}$ order & 249.185 & 19.251 & 214.141 & 90.087 \\
\hline
\end{tabular}

Evidently, the linear model prediction error (as compared to the nonlinear dynamic simulator) varies significantly among the different linear approximations. This demonstrates that Eq. 19 is useful to provide insight concerning the low performing linear models among each plant's set. Without comparison to reference trajectories, gaining this 
insight may be difficult. For instance, it was found out that "fit to model" data is a poor predictor of linearisation error for this case study, since the step test model identification provided a good fit to model data (always over $80 \%$, reaching up to $99.9 \%$ in some cases) for the models of all input/output pairs. Thus, the significant prediction error in comparison to the nonlinear dynamic simulator is mostly due to process nonlinearity, not the standard step test identification procedure that provided state-space model matrices $\mathrm{A}, \mathrm{B}, \mathrm{C}$ and D.

Let us now analyse the performance of each SMLP method and effects of the choice of polynomial order to be used as SMLP degradation functions. First, it is important to notice that, due to their design, plant 3 and 4 have slower dynamics leading to lesser $\mathrm{CV}$ variation and overall error in the same timeframe. Thus, the error should not be compared across plants, but between the single sub-models and SMLP models of the same plant. That said all SMLP variants performed better than their respective linear submodels, with smaller prediction error relative to the training dataset. In most instances, there were accuracy benefits in increasing the order of the degradation functions, except for SMLP methods 2 and 3 for plant 2, for which the $3^{\text {rd }}$ order was optimal instead of the $4^{\text {th }}$. As an important downside, the time necessary to solve the regression analysis is tripled or quadrupled each time the order is increased by 1 .

Now it is necessary to test the SMLP models using the validation data. As explained in section 3.2, this is a useful procedure to detect overfitting and check the overall model robustness. Table 10 presents values for the sum of normalised squared error between the validation dataset, the linear sub-models and the SMLP models, through the prediction horizon.

Table 10 - Residue from regression analysis for the SMLP problem using the training dataset (Set 2) as a reference.

\begin{tabular}{|c|c|c|c|c|}
\hline Total normalised squared error & Plant 1 & Plant 2 & Plant 3 & Plant 4 \\
\hline Linear sub-model $1\left(\right.$ IP $\left._{\mathbf{1}}\right)$ & 36.685 & 49.492 & 87.410 & 21.936 \\
\hline Linear sub-model $2\left(I_{2}\right)$ & 80.001 & 47.683 & 46.615 & 243.055 \\
\hline Linear sub-model $3\left(\mathrm{IP}_{3}\right)$ & 101.812 & 25.612 & 181.551 & 28.353 \\
\hline SMLP Method $1-2^{\text {nd }}$ order & 35.513 & 24.392 & 32.321 & 63.105 \\
\hline SMLP Method $1-3^{\text {rd }}$ order & 35.433 & 23.919 & 31.782 & 96.915 \\
\hline SMLP Method $1-4^{\text {th }}$ order & 35.423 & 23.925 & 31.771 & 100.947 \\
\hline SMLP Method $2-2^{\text {nd }}$ order & 36.339 & 19.929 & 31.550 & 19.620 \\
\hline SMLP Method $2-3^{\text {rd }}$ order & 36.198 & 20.616 & 32.476 & 19.455 \\
\hline SMLP Method $2-4^{\text {th }}$ order & 35.531 & 21.511 & 32.478 & 19.565 \\
\hline SMLP Method $3-2^{\text {nd }}$ order & 30.017 & 19.606 & 26.659 & 39.985 \\
\hline
\end{tabular}




\begin{tabular}{|l|l|l|l|l|}
\hline SMLP Method $3-3^{\text {rd }}$ order & 30.963 & 18.837 & 25.960 & 56.002 \\
\hline SMLP Method $3-4^{\text {th }}$ order & 32.046 & 19.251 & 26.081 & 45.618 \\
\hline
\end{tabular}

For the SMLP method 1 (output-based IPs) the gains accuracy obtained through the order increase were very modest for plants 1,2 and 3. Also, the phenomenon of overfitting is evident for plant 4 , for which happened very large increases in model error as related to the reference data. It is important to point out that, while for plants 1, 2 and 3 , method 1 managed to reduce prediction error relative to any of the three single linear models that compose the SMLP; it actually increased error for plant 4.

The SMLP method 2 (MIMO input-based IPs) fared much better for plant 4, consistently reducing error as compared to any of the single models, and somewhat better for plant 2. For plants 1 and 3, results were equivalent to those obtained from method 1. Degradation functions order increase caused modest gains for plant 1 , with the $4^{\text {th }}$ order being optimal, and some small degradation for the others (the $3^{\text {rd }}$ optimal order was optimal for plant $4,2^{\text {nd }}$ order for plants 2 and 3). SMLP method 3 (SISO input-based IPs) had the lowest prediction error for plants 1, 2 and 3, and intermediate results for plant 4, for which again strong overfitting can be observed.

For this case study, the sensible choice is, for each plant, to select the SMLP method that presents the smallest average prediction error between the two reference trajectories (training and validation datasets). We advise against taking the absolute values in Table 10 and comparing them to those of Table 9 since the reference trajectories are very different. Hence, geometrical averages instead of arithmetical are used. Results are presented in Table 11.

Table 11 - Geometric averaged normalised squared error between the two reference trajectories (training and validation datasets).

\begin{tabular}{|c|c|c|c|c|}
\hline $\begin{array}{c}\text { Geometric averaged } \\
\text { normalised squared error }\end{array}$ & Plant 1 & Plant 2 & Plant 3 & Plant 4 \\
\hline Error m1 & 107.48 & 67.61 & 191.34 & 76.43 \\
Error m2 & 192.06 & 79.01 & 120.24 & 541.73 \\
Error m3 & 250.08 & 127.90 & 337.54 & 68.57 \\
\hline Error SMLP Method 1 2nd order & 102.01 & 34.51 & 96.21 & 68.64 \\
Error SMLP Method 1 3rd order & 101.89 & 34.16 & 95.28 & 84.89 \\
Error SMLP Method 1 4th order & 101.86 & 34.17 & 95.26 & 86.25 \\
\hline Error SMLP Method 2 2nd order & 102.94 & 30.01 & 95.87 & 41.53 \\
Error SMLP Method 2 3rd order & 102.69 & 30.16 & 97.26 & 41.06 \\
Error SMLP Method 2 4th order & 101.44 & 30.57 & 97.26 & 40.77 \\
\hline Error SMLP Method 3 2nd order & 87.21 & 28.11 & 76.75 & 60.77 \\
Error SMLP Method 3 3rd order & 88.12 & 26.94 & 74.61 & 71.03
\end{tabular}




\begin{tabular}{|l|l|l|l|l|} 
Error SMLP Method 3 4rd order & 89.36 & 27.18 & 74.73 & 64.11 \\
\hline
\end{tabular}

As can be seen, the lowest averaged prediction error was presented by SMLP method 3 for plant 1, 2 and 3, and method 2 for plant 4, when the degradation function was of $2^{\text {nd }}$ order for plant $1,3^{\text {rd }}$ order for plant 2 and 3 , and $4^{\text {th }}$ order for plant 4 . These SMLP models were thus selected to evaluate plant design using the EMOP index methodology, as discussed in section 5 .

\subsection{Results and Discussion}

In this Section, the results obtained through the application of the EMOP to assessing alternative distillation plant designs are presented.

\subsubsection{Scenario 1 - Simultaneous control and optimisation while handling a measured disturbance}

Table 12 - Initial values for process outputs.

\begin{tabular}{cccccccccccc}
\hline & $\mathbf{y}_{\mathbf{1}}$ & $\mathbf{y}_{\mathbf{2}}$ & $\mathbf{y}_{\mathbf{3}}$ & $\mathbf{y}_{\mathbf{4}}$ & $\mathbf{y}_{\mathbf{5}}$ & $\mathbf{y}_{\mathbf{6}}$ & $\mathbf{y}_{\mathbf{7}}$ & $\mathbf{y}_{\mathbf{8}}$ & $\mathbf{y}_{\mathbf{9}}$ & $\mathbf{y}_{\mathbf{1 0}}$ & $\mathbf{y}_{\mathbf{1 1}}$ \\
\hline Case 1 & 46.00 & 81.76 & 288.81 & 319.42 & 341.63 & -25.47 & 826.79 & 251.11 & 49.79 & 812.88 & -68.38 \\
\hline
\end{tabular}

Table 13 - Initial values for process inputs.

\begin{tabular}{ccccccccc}
\hline & $\mathbf{u}_{\mathbf{1}}$ & $\mathbf{u}_{\mathbf{2}}$ & $\mathbf{u}_{\mathbf{3}}$ & $\mathbf{u}_{\mathbf{4}}$ & $\mathbf{u}_{\mathbf{5}}$ & $\mathbf{u}_{\mathbf{6}}$ & $\mathbf{u}_{\mathbf{7}}$ & $\mathbf{d}_{\mathbf{1}}$ \\
\hline Case 1 & 42.64 & 335.13 & 135.75 & 25.71 & 550.00 & 250.00 & 0.00 & 0.00 \\
\hline
\end{tabular}

In this first scenario, all variables are at first within their control zones as shown in Table 12 , but the process is going to be disturbed by an increase in the slop feed to the process, which is raised from 0 to $40 \mathrm{~m}^{3} / \mathrm{h}$. The rate of increase will be $24\left(\mathrm{~m}^{3} / \mathrm{h}\right) / \mathrm{h}$, so the value is achieved in $1 \mathrm{~h} 40 \mathrm{~m}$. The MVs in Table 14 (only final values are shown) were found to be the best available for each plant, resulting in the CVs values for each plant provided in Table 15.

Table 14 - Inputs for the scenario $1 .{ }^{*} \mathrm{u}_{3}+\mathrm{u}_{4} \geq 140 ; * * \mathrm{u}_{5}+\mathrm{u}_{6}+\mathrm{u}_{7}+\mathrm{d}_{1}=800$.

\begin{tabular}{lccccccccc}
\hline & $\begin{array}{c}\text { Manipulated Variables } \\
\text { Description }\end{array}$ & Unit & Max. & Min. & Initial & \multicolumn{4}{c}{ Final Value } \\
\cline { 7 - 11 } & Value & Value & Value & Plant & Plant & Plant & Plant \\
$\mathbf{\mathbf { u } _ { \mathbf { 1 } }}$ & Temperature 01 tray TIC01.SP & $\mathrm{C}$ & 70.0 & 40.0 & 42.64 & 41.62 & 62.27 & 43.40 & 53.93 \\
$\mathbf{u}_{\mathbf{2}}$ & Temperature Fired Heater TIC02.SP & $\mathrm{C}$ & 380.0 & 320.0 & 335.13 & 333.60 & 351.53 & 339.67 & 343.81 \\
\hline $\mathbf{u}_{\mathbf{3}}$ & Light Diesel Output FC02.SP & $\mathrm{m}^{3} / \mathrm{h}$ & 270.0 & $0.0(*)$ & 135.75 & 119.65 & 108.25 & 136.45 & 103.88 \\
$\mathbf{u}_{\mathbf{4}}$ & Heavy Diesel Output FC03.SP & $\mathrm{m}^{3} / \mathrm{h}$ & 65.0 & $0.0(*)$ & 25.71 & 22.77 & 33.32 & 27.73 & 36.18 \\
\hline $\mathbf{u}_{\mathbf{5}}$ & Medium Crude Flow Rate FC01A.SP & $\mathrm{m}^{3} / \mathrm{h}$ & $800(* *)$ & 0.0 & 550.00 & 607.03 & 327.26 & 490.92 & 154.35 \\
$\mathbf{u}_{\mathbf{6}}$ & Light Crude Flow Rate FC01B.SP & $\mathrm{m}^{3} / \mathrm{h}$ & $800(* *)$ & 0.0 & 250.00 & 127.24 & 194.25 & 180.22 & 133.38 \\
\hline $\mathbf{u}_{\mathbf{7}}$ & Heavy Crude Flow Rate FC01C.SP & $\mathrm{m}^{3} / \mathrm{h}$ & $800(* *)$ & 0.0 & 0.00 & 25.72 & 238.49 & 88.86 & 472.27 \\
$\mathbf{d}_{\mathbf{1}}$ & Slop Oil Feed Recycling Flow Rate & $\mathrm{m}^{3} / \mathrm{h}$ & 0.00 & 0.00 & 0.00 & 40.00 & 40.00 & 40.00 & 40.00 \\
\hline
\end{tabular}

Table 15 - Output predictions for the scenario 1 .

\begin{tabular}{|c|c|c|c|c|c|c|c|c|}
\hline \multirow[b]{2}{*}{ Controlled Variables Description } & \multirow[b]{2}{*}{ Unit } & \multirow{2}{*}{$\begin{array}{l}\text { Min. } \\
\text { Value }\end{array}$} & \multirow{2}{*}{$\begin{array}{l}\text { Max. } \\
\text { Value }\end{array}$} & \multirow{2}{*}{$\begin{array}{l}\text { Initial } \\
\text { Value }\end{array}$} & \multicolumn{4}{|c|}{ Final Value } \\
\hline & & & & & $\begin{array}{c}\text { Plant } \\
1\end{array}$ & $\begin{array}{c}\text { Plant } \\
2\end{array}$ & $\begin{array}{c}\text { Plant } \\
\mathbf{3}\end{array}$ & $\begin{array}{c}\text { Plant } \\
4\end{array}$ \\
\hline
\end{tabular}




\begin{tabular}{lccccccccc}
\hline $\mathbf{y}_{\mathbf{1}}$ & Cetane Index DIESEL & & 46.0 & - & 46.00 & $\mathbf{5 0 . 0 0}$ & 51.30 & 50.38 & 48.65 \\
$\mathbf{y}_{\mathbf{2}}$ & Flash Point DIESEL & $\mathrm{C}$ & 55.0 & - & 81.76 & 87.82 & 87.90 & 88.87 & 99.90 \\
$\mathbf{y}_{\mathbf{3}}$ & ASTM D86 DIESEL 65\% & $\mathrm{C}$ & 250.0 & - & 288.81 & 311.02 & 284.63 & 302.40 & 304.33 \\
$\mathbf{y}_{\mathbf{4}}$ & ASTM D86 DIESEL 85\% & $\mathrm{C}$ & - & 350.0 & 319.42 & 342.30 & 306.39 & 344.11 & 332.24 \\
$\mathbf{y}_{\mathbf{5}}$ & ASTM D86 DIESEL 95\% & $\mathrm{C}$ & - & 370.0 & 341.63 & 369.34 & 366.79 & $\mathbf{3 7 0 . 0 0}$ & 363.79 \\
$\mathbf{y}_{\mathbf{6}}$ & Freezing Point DIESEL & $\mathrm{C}$ & - & -15.0 & -25.47 & -31.21 & $\mathbf{- 1 5 . 0 0}$ & -15.95 & $\mathbf{- 1 5 . 0 0}$ \\
$\mathbf{y}_{\mathbf{7}}$ & Density (15 C) DIESEL & $\mathrm{kg} / \mathrm{m}^{3}$ & 820.0 & 860.0 & 826.79 & 831.96 & 842.74 & 831.32 & $\mathbf{8 6 0 . 0 0}$ \\
$\mathbf{y}_{\mathbf{8}}$ & ASTM D86 KEROSENE 100\% & $\mathrm{C}$ & - & 300.0 & 251.11 & 265.04 & 271.31 & 251.88 & 275.36 \\
$\mathbf{y}_{\mathbf{9}}$ & Flash Point KEROSENE & $\mathrm{C}$ & 38.0 & - & 49.79 & 52.43 & 49.95 & 75.21 & 43.30 \\
$\mathbf{y}_{\mathbf{1 0}}$ & Density (15 C) KEROSENE & $\mathrm{kg} / \mathrm{m}^{3}$ & 775.0 & 840.0 & 812.88 & 817.53 & 827.33 & 822.11 & 837.61 \\
$\mathbf{y}_{\mathbf{1 1}}$ & Freezing Point KEROSENE & $\mathrm{C}$ & - & -47.0 & -68.38 & -67.09 & -63.58 & -53.61 & -61.94 \\
\hline
\end{tabular}

For these sets of process inputs, the optimised cost function values for each plant are provided in Table 16.

Table 16 - Cost function values for each plant - scenario 1.

\begin{tabular}{cccccc}
\hline & Plant 1 & Plant 2 & Plant 3 & Plant 4 & Unit \\
\hline $\begin{array}{c}\text { EMOP Index } \\
\text { Scenario 1 }\end{array}$ & 11609 & 11094 & 10042 & 13066 & US\$/h \\
\hline
\end{tabular}

All plants managed to keep CVs within control bounds. Three factors explain the difference observed in index values: the mix of crude oil fed into the plant, the quality (Cetane Index) of diesel oil produced and, to a lesser degree, the energy spent in the fired heater. Increasing the percentage of light oil makes it easier to meet quality requirements but increase costs, while adding more heavy oil has the contrary effect: it decreases the quality while cutting raw materials cost. Lower values for EMOP index are better and thus plant 3 presented the best performance through a favourable ratio between heavy and light crude, and low energy consumption. It was closely followed by plant 2 , which processed more heavy crude but also more light crude. Plant 1 was a little behind, using the lesser amount of expensive light oil but also almost no cheap heavy oil.

Plants 1, 2 and 3 produced premium priced diesel with 50 or more Cetane Index (CI), which improved the EMOP index as compared to plant 4. Plant 4's performance was acceptable but not as good as the others. It used a great amount of heavy oil instead of medium oil, which is good, but its diesel output did not meet the $50 \mathrm{CI}$ bound. Hence, it would need to be marketed instead as $48 \mathrm{CI}$ diesel, reducing profitability.

It is also important to look at which $\mathrm{CV}$ s are active, i.e., acting as optimisation constraints for each plant. The quality threshold $\mathrm{y}_{1}(\mathrm{CI})$ (see the revised SMLP index in section 5) was active for plant 1 . This means that plant 1 was limited by the control effort needed to attain the upper-quality threshold of $\mathrm{y}_{1}>50$. On the other hand, $\mathrm{y}_{6}$ (diesel freezing point) reached the upper bound of its control zone for plants 2 and 4, preventing further optimisation, whereas $\mathrm{y}_{5}(95 \%$ boiling point) limited gains for plants 3 . It comes 
to attention that $\mathrm{y}_{7}$ was an active constraint for plant 4 , which may be explained by the large percentage of heavy oil processed by it.

\subsubsection{Scenario 2 - Price changes}

In this second scenario, changes were made to the crude oil and diesel prices and the process optimisation is resumed at the same state where each plant was at the end of scenario 1, meaning that each plant has a different starting point. How favourable this new starting point depends on the flowsheets themselves and their performance in the first scenario. The sale prices of all diesel oil variants were increased in 5\%, and the costs of Medium and Light crudes were increased in $4 \%$ and $2 \%$ respectively, whereas the flowrate of expensive heavy Crude was lowered in $11 \%$. The new values are provided in Table 17:

Table 17 - Crude Oil Costs and Product Prices.

\begin{tabular}{|c|c|c|}
\hline Stream & $\mathrm{US} \$ / \mathbf{m}^{3}$ & Price \\
\hline Medium Crude Oil & $\mathrm{US} \$ / \mathrm{m}^{3}$ & 447.26 \\
\hline Heavy Crude Oil & $\mathrm{US} \$ / \mathrm{m}^{3}$ & 354.41 \\
\hline Light Crude Oil & $\mathrm{US} \$ / \mathrm{m}^{3}$ & 481.36 \\
\hline Diesel - CI 46 & $\mathrm{US} \$ / \mathrm{m}^{3}$ & 578.77 \\
\hline Diesel - CI 48 & $\mathrm{US} \$ / \mathrm{m}^{3}$ & 592.70 \\
\hline Diesel - CI 50 & $\mathrm{US} \$ / \mathrm{m}^{3}$ & 613.07 \\
\hline
\end{tabular}

Also, the process is to be disturbed by a decrease in the slop feed to the process from 40 to $20 \mathrm{~m}^{3} / \mathrm{h}$, and the decrease rate will be of $12\left(\mathrm{~m}^{3} / \mathrm{h}\right) / \mathrm{h}$. Once again, the final flow is achieved in $1 \mathrm{~h} 40 \mathrm{~m}$. The optimisation was carried out keeping all remaining parameters constant, providing the MVs in Table 18 and the CVs in 
Table 19:

Table 18 - Inputs for the scenario $1 . * \mathrm{u}_{3}+\mathrm{u}_{4} \geq 140 ; * * \mathrm{u}_{5}+\mathrm{u}_{6}+\mathrm{u}_{7}+\mathrm{d}_{1}=800$; $* * *$ Initial MVs are the final values in Table 7.

\begin{tabular}{|c|c|c|c|c|c|c|c|c|c|}
\hline & \multirow[b]{2}{*}{$\begin{array}{c}\text { Manipulated Variables } \\
\text { Description }\end{array}$} & \multirow[b]{2}{*}{ Unit } & \multirow[b]{2}{*}{$\begin{array}{l}\text { Max. } \\
\text { Value }\end{array}$} & \multirow[b]{2}{*}{$\begin{array}{l}\text { Min. } \\
\text { Value }\end{array}$} & \multirow[b]{2}{*}{$\begin{array}{l}\text { Initial } \\
\text { Value }\end{array}$} & \multicolumn{4}{|c|}{ Final Value } \\
\hline & & & & & & $\begin{array}{c}\text { Plant } \\
1\end{array}$ & $\begin{array}{c}\text { Plant } \\
2\end{array}$ & $\begin{array}{c}\text { Plant } \\
3\end{array}$ & $\begin{array}{c}\text { Plant } \\
4\end{array}$ \\
\hline $\mathbf{u}_{1}$ & Temperature 01 tray TIC01.SP & $\mathrm{C}$ & 70.0 & 40.0 & $* * *$ & 45.27 & 58.89 & 42.66 & 53.51 \\
\hline $\mathbf{u}_{2}$ & Temperature Fired Heater TIC02.SP & $\mathrm{C}$ & 380.0 & 320.0 & $* * *$ & 339.96 & 356.18 & 342.00 & 342.08 \\
\hline $\mathbf{u}_{3}$ & Light Diesel Output FC02.SP & $\mathrm{m} 3 / \mathrm{h}$ & 270.0 & $0.0(*)$ & $* * *$ & 108.36 & 130.12 & 132.07 & 98.63 \\
\hline $\mathbf{u}_{4}$ & Heavy Diesel Output FC03.SP & $\mathrm{m} 3 / \mathrm{h}$ & 65.0 & $0.0(*)$ & $* * *$ & 29.97 & 43.13 & 28.34 & 41.37 \\
\hline $\mathbf{u}_{\mathbf{5}}$ & Medium Crude Flow Rate FC01A.SP & $\mathrm{m} 3 / \mathrm{h}$ & $800(* *)$ & 0.0 & $* * *$ & 545.90 & 403.11 & 491.33 & 166.39 \\
\hline $\mathbf{u}_{6}$ & Light Crude Flow Rate FC01B.SP & $\mathrm{m} 3 / \mathrm{h}$ & $800(* *)$ & 0.0 & $* * *$ & 115.47 & 145.49 & 147.43 & 124.56 \\
\hline $\mathbf{u}_{7}$ & Heavy Crude Flow Rate FC01C.SP & $\mathrm{m} 3 / \mathrm{h}$ & $800(* *)$ & 0.0 & *** & 118.63 & 231.40 & 141.24 & 489.05 \\
\hline$d_{1}$ & Slop Oil Feed Recycling Flow Rate & $\mathrm{m} 3 / \mathrm{h}$ & 0.00 & 0.00 & **** & 20.00 & 20.00 & 20.00 & 20.00 \\
\hline
\end{tabular}


Table 19 - Output predictions for the scenario 2.* Initial CVs are the final values in Table 8.

\begin{tabular}{|c|c|c|c|c|c|c|c|c|c|}
\hline & \multirow[b]{2}{*}{ Controlled Variables Description } & \multirow[b]{2}{*}{ Unit } & \multirow{2}{*}{$\begin{array}{l}\text { Min. } \\
\text { Value }\end{array}$} & \multirow{2}{*}{$\begin{array}{l}\text { Max. } \\
\text { Value }\end{array}$} & \multirow{2}{*}{$\begin{array}{l}\text { Initial } \\
\text { Value }\end{array}$} & \multicolumn{4}{|c|}{ Final Value } \\
\hline & & & & & & $\begin{array}{c}\text { Plant } \\
1\end{array}$ & $\begin{array}{c}\text { Plant } \\
2\end{array}$ & $\begin{array}{c}\text { Plant } \\
3\end{array}$ & $\begin{array}{c}\text { Plant } \\
4\end{array}$ \\
\hline $\mathbf{y}_{1}$ & Cetane Index DIESEL & & 46.0 & - & $*$ & 50.00 & 50.00 & 50.00 & 48.73 \\
\hline $\mathbf{y}_{2}$ & Flash Point DIESEL & $\mathrm{C}$ & 55.0 & - & $*$ & 89.65 & 83.63 & 90.20 & 98.47 \\
\hline $\mathbf{y}_{3}$ & ASTM D86 DIESEL 65\% & $\mathrm{C}$ & 250.0 & - & $*$ & 314.72 & 297.00 & 294.81 & 303.92 \\
\hline $\mathbf{y}_{4}$ & ASTM D86 DIESEL 85\% & $\mathrm{C}$ & - & 350.0 & * & 344.10 & 332.14 & 347.03 & 334.13 \\
\hline $\mathbf{y}_{5}$ & ASTM D86 DIESEL 95\% & $\mathrm{C}$ & - & 370.0 & $*$ & 369.68 & 368.04 & 368.97 & 358.92 \\
\hline $\mathbf{y}_{6}$ & Freezing Point DIESEL & $\mathrm{C}$ & - & -15.0 & * & -20.40 & -15.01 & -15.96 & -15.00 \\
\hline $\mathbf{y}_{7}$ & Density (15 C) DIESEL & $\mathrm{kg} / \mathrm{m}^{3}$ & 820.0 & 860.0 & $*$ & 838.07 & 840.49 & 832.65 & 860.00 \\
\hline $\mathbf{y}_{8}$ & ASTM D86 KEROSENE 100\% & $\mathrm{C}$ & - & 300.0 & $*$ & 254.04 & 272.39 & 251.84 & 273.33 \\
\hline $\mathbf{y}_{9}$ & Flash Point KEROSENE & $\mathrm{C}$ & 38.0 & - & $*$ & 61.43 & 46.22 & 80.06 & 38.86 \\
\hline $\mathbf{y}_{10}$ & Density (15 C) KEROSENE & $\mathrm{kg} / \mathrm{m}^{3}$ & 775.0 & 840.0 & $*$ & 821.21 & 823.96 & 824.73 & 835.07 \\
\hline $\mathbf{y}_{11}$ & Freezing Point KEROSENE & $\mathrm{C}$ & - & -47.0 & $*$ & -61.97 & -72.66 & -50.97 & -62.88 \\
\hline
\end{tabular}

For these sets of process inputs, the cost function values for each plant are provided in Table 20.

Table 20 - Cost function values for each plant - scenario 2 .

\begin{tabular}{cccccc}
\hline & Plant 1 & Plant 2 & Plant 3 & Plant 4 & Unit \\
\hline $\begin{array}{c}\text { EMOP Index } \\
\text { Scenario 2 }\end{array}$ & 16771 & 11180 & 14321 & 13655 & US\$/h \\
\hline
\end{tabular}

Again, all CVs were kept within control zones, and plant 2 presented the best EMOP index by using a favourable oil mix (with a significant amount of heavy crude and low light crude) and producing $50 \mathrm{CI}$ diesel. This time plant 4, which again processed a large ratio of heavy oil, had an EMOP index a little lower than plant 3 and significantly lower than plant 1 , even if it did not manage to produce $50 \mathrm{CI}$ diesel.

Plant 1, 2 and 3 reached the diesel CI quality threshold, $\mathrm{y}_{1}=50$, being constrained by it. In addition to that, $\mathrm{y}_{5}$ acted as an active restriction for plants 2 and 4 , and, once again, $\mathrm{y}_{7}$ was an active constraint for plant 4 .

\subsubsection{Selecting the Best Plant}

The results obtained in both scenarios must be considered together in order to draw a consistent conclusion about which plant has the better characteristics when it comes to MPC zone control. The average EMOP index used in this Section was defined as the geometric mean of each plant's indices multiplied by the weight for each scenario, which was set at 1 for both. The geometric mean is used instead of the arithmetic mean as a way to penalise plant designs with particularly poor performance in one or more scenarios. Using the arithmetic mean could hide an issue if several scenarios are tested. 
Table 21 - Average mean EMOP index for each plant.

\begin{tabular}{ccccccc}
\hline & Plant 1 & Plant 2 & Plant 3 & Plant 4 & Unit & Case Weight \\
\hline Case 1 & 11609 & 11094 & 10042 & 13066 & US\$/h & 1 \\
Case 2 & 16771 & 11180 & 14321 & 13655 & US\$/h & 1 \\
Geometric Mean & 13953 & 11137 & 11992 & 13357 & US\$/h & \\
\hline
\end{tabular}

As can be seen in 
Table 21, plant 2 had the best overall results in this application of the EMOP index and should provide better MPC controllability than the alternative flowsheets. However, plant 3 had reasonably close results. In a comprehensive analysis, including capital and maintenance costs, it may be that plant 2 is the sensible choice.

One should notice that plant 1 and 2 are identical and one can interpret the performance difference between them because of distinct controllability. Plant 2 has improved controllability due to the location where the diesel and kerosene analysers are placed, i.e., downstream of product tanks, as opposed to upstream in plant 1.

This analysis demonstrated that the choice of instrument location for plant 1 is had an adverse impact on process dynamics and control performance, and it should be scrapped in favour of the solution adopted for plant 2 . This case study also demonstrated that the selection of a suboptimal flowsheet could increase OPEX from $7 \%$ to $25 \%$ relative to the optimal flowsheet. Even the lower range of this figure translates into expressive amounts of money being wasted, especially given the high revenue and long life-cycles of the chemical industry's projects. It should be noted that while here only two scenarios were tested for a single disturbance, for the design of industrial plants many more possibilities should be investigated for a comprehensive analysis.

\subsection{Choosing between Multiple and Single State-Space Models - the Impact on the Integrated Process Design and Control Approach}

Comparing these results to those obtained whilst assessing the same flowsheets in Strutzel and Bogle (2016), two striking differences appear: the index of plant 4, which was the worst performer in the previous analysis, now stands between plant 1 and 3 . In addition, the assessment of plant 2 was very poor. In that previous paper, it was considered the worst plant, and now it stands out as the best. Representing plant 2 as an SMLP system presented a far more accurate EMOP index.

The quality of the assessment done previously for this flowsheet was poor due to its strongly nonlinear gain. It is important to notice that this nonlinearity came from the nonlinear model (or real plant) itself: even if the procedure to identify a linear model is well done and the resulting model is good at predicting trajectories close to its identification point, it may or may not be useful at other operation points. Therefore, model nonlinearity is indeed strong enough to cause single linear models to be inadequate 
for Integrated Process Design and Control approaches carried out for separation processes and, very likely, also for reactional processes.

\section{Conclusions}

The simultaneous multi-linear prediction (SMLP) method becomes an interesting option when it is desired to obtain linear approximations of an unknown nonlinear model relying just on its numerical output. Moreover, the SMLP is recommended when the number of available linearisations (sub-models) is limited but the expected operational range is considerably wide. Thus, the SMLP brings performance improvements in some frequent and important situations: when commercial, closed source dynamic simulation packages are to be used for designing new plants, or when an existing plant is to be modelled for MPC. The models show that the SMLP can greatly reduce the inherent error incurred when using linearised models. It was shown that SMLP systems can be applied in the fields of control, dynamic analysis and, as demonstrated in this paper, integrated process design and control.

The main alternative to using an SMLP system would be a multi-model PWA system. Nevertheless, for the typical SMLP use scenario, this may not be as good an option. The issue is that switching the state update rules based on arbitrary, fixed thresholds may not improve the quality of the multi-model prediction. This is true because a linear sub-model of a piecewise affine system might not be more accurate than the rest in the entirety of its region, i.e., there is no guarantee of obtaining an optimal partition. But even optimal partitions can sometimes be a problem. Sudden and possibly drastic model transitions may happen as the OP crosses a threshold. This issue is normally minimised by the introduction of restrictions to obtain smoother transitions between the region boundaries, but this reduces the fidelity of the approximation. Avoiding this tradeoff, the use of SMLP shifts the priority among sub-models continuously, without the need for boundaries as a means to achieve a more faithful reproduction of the nonlinear model. No threshold or partition rule is used and changes in the output update equation are likely to be smoother. The SMLP regression analysis is unconstrained and solely focused on reducing model mismatch. Also, the SMLP method does not require the use of mixedinteger linear programming (MILP), allowing the use of faster optimisation algorithms.

SMLP systems can be used to evaluate expected flowsheet control performance for integrated process design and control. In the case study provided, an SMLP system 
was assembled to predict the dynamic behaviour of a crude oil distillation plant. This provided the basis successfully for an application of the Economic MPC optimisation (EMOP) index presented in Strutzel and Bogle (2016). Hence, by using the EMOP index together with the SMLP, the former became more precise and accurate in its analysis of the economic impact of the MPC control effort.

A future development may introduce a mixed approach using iteratively both the SMLP and a nonlinear, closed source model to provide dynamic predictions for an Integrated Process Design and Control analysis.

\section{Acknowledgement}

The authors thank Petrobras and University College London for the encouragement and support received. 


\section{References}

Agarwal P, Afshar Alam M, Biswas R. Challenges and Tools of Clustering Algorithms. Int J Comp Sci Issues 2011, 8(3):523-528.

Baccelli F, Cohen G, Olsder G, Quadrat J. Synchronization and Linearity. Wiley, 1992.

Bellman R. Adaptive Control Processes: A Guided Tour, Princeton University Press, Princeton, New Jersey (1961).

Bemporad A, Garulli A, Paoletti S, Vicino A. A bounded-error approach to piecewise affine system identification. IEEE Transactions on Automatic Control 2005;50(10):1567-80.

Bemporad A, Morari M: Control of systems integrating logic, dynamics, and constraints. Automatica 1999, 35(3):407-27.

Christophersen FJ. Optimal Control and Analysis for Constrained Piecewise Affine Systems. Dissertation ETH Zurich No. 16807.

Cottle RW, Pang JS, Stone RE. The Linear Complementarity Problem, Academic Press, Cambridge, 1992.

Cuninghame-Green R. Minimax Algebra. Springer, 1979.

De Schutter B, Van den Boom T. On model predictive control for max-min-plus-scaling discrete event systems. Automatica 2001, 37(7):1049-56.

Dougherty D, Cooper D. A practical multiple model adaptive strategy for single-loop MPC. Control Engineering Practice 2003:11:141-159.

Edgar TF. Control and operations: when does controllability equal profitability? Comput Chem Eng 2004;29:41-49.

Everitt, B. S. Cluster Analysis: A Brief Discussion of Some of the Problems. Brit J Psychiat 1972, 120:143-5.

Everitt, B. S. Cluster Analysis. Heinemann 1974, London.

Ferrari-Trecate G, Muselli M, Liberati D, Morari M. A clustering technique for the identification of piecewise affine systems. Automatica 2003, 39(2):205-17.

Francisco M, Vega P, Alvarez H. Robust integrated design of process with terminal penalty model predictive controllers. Ind Eng Chem Res 2011;89:1011-24.

Gareth J. An Introduction to Statistical Learning: with Applications in R. Springer, 2013:176. ISBN 978-1461471370.

Garulli A, Paoletti S, Vicino A. A survey on switched and piecewise affine system identification. 16th IFAC Symposium on System Identification 2012; The International Federation of Automatic Control.

Gelb A. Applied Optimal Estimation. MIT Press, 1974.

Gopinath, R., Bequette, B. W., Roy, R. J., Kaufman, H., \& Yu, C. Issues in the design of a multirate model-based controller for a nonlinear drug infusion system. Biotechnology Progress 1995;11:318-32.

Hautus M. Controllability and stabilizability of sampled systems. IEEE Transactions on Automatic Control 1972;17(4):528-531. 
Heemels WPMH, Schumacher JM, Weiland S. Linear complementarity systems. SIAM J Appl Math 2000, 60(4):1234-69.

Heemels WPMH, De Schutter B, Bemporad A. Equivalence of hybrid dynamical models. Automatica 2001, 37(7):1085-91.

Kalman RE, Falb PL. Arbib MA. Topic in Mathematical System Theory, MacGraw-Hill Book Company, New York, 1969.

Kantner M, Primbs J. Nonlinear MPC Lower Bounds via Robust Simulation. Proceedings of the American Control Conference Albuquerque 1997, New Mexico.

Lee JH, Morari M, Garcia CE. State-space interpretation of model predictive control. Automatica 1994;30(4):707-17.

Juloski ALj, Weiland S, Heemels WPMH. A Bayesian approach to identification of hybrid systems. IEEE Transactions on Automatic Control 2005;50(10):1520-33.

Lowe GK, Zohdy MA. Modeling nonlinear systems using multiple piecewise linear equations. Nonlinear Analysis: Modelling and Control 2010;15(4):451-58.

Lyapunov AM. The General Problem of the Stability of Motion (In Russian), Doctoral dissertation, Univ. Kharkov 1892 English translations: (1) Stability of Motion, Academic Press, New-York \& London, 1966 (2) The General Problem of the Stability of Motion, (A. T. Fuller trans.) Taylor \& Francis, London 1992.

Nakada H, Takaba K, Katayama T. Identification of piecewise affine systems based on statistical clustering technique. Automatica, 41(5):905-913, 2005.

Paoletti S, Juloski A Lj. , G. Ferrari-Trecate, and R. Vidal. Identification of hybrid systems: A tutorial. European Journal of Control 2007;13(2-3):242-60.

Roll J. Local and Piecewise Affine Approaches to System Identification. $\mathrm{PhD}$ thesis, Department of Electrical Engineering, Link"oping University, Linkoping, Sweden, 2003. http://www.control.isy.liu.se/publications/.

Roll J, Bemporad A, Ljung L. Identification of piecewise affine systems via mixedinteger programming. Automatica 2004, 40:37-50.

Sontag ED. Nonlinear regulation: The piecewise linear approach. IEEE Trans. on Automatic Control 1981, 26(2):346-58.

Strutzel FAM, Bogle IDL. Assessing plant design with regard to MPC performance. Comput Chem Eng 2016;94:180-211.

Tikhonov AN. O решении некорректно поставленных задач и методе регуляризации". Doklady Akademii Nauk SSSR 1963. 151:501-504. Translated in "Solution of incorrectly formulated problems and the regularization method". Soviet Mathematics. 4: 1035-1038.

Vidal R, Soatto S, Ma Y, Sastry S. An algebraic geometric approach to the identification of a class of linear hybrid systems. In Proc. 42nd IEEE Conference on Decision and Control 2003:167-172. 


\section{Appendix}

\section{Continuous-Time Transfer Functions for the Crude Oil Distillation Process}

Table 22 to Table 45 display parameters to be substituted in Eq. 90 to represent each model as a 5th order transfer function, which can be easily converted to any statespace formulation.

$$
G_{i, j}(s)=\frac{b_{i, j, 0}+b_{i, j, 1} s+b_{i, j, 2} s^{2}+b_{i, j, 3} s^{3}+b_{i, j, 4} s^{4}+b_{i, j, 5} s^{5}}{a_{i, j, 0}+a_{i, j, 1} s+a_{i, j, 2} s^{2}+a_{i, j, 3} s^{3}+a_{i, j, 4} s^{4}+a_{i, j, 5} s^{5}}
$$

Eq. 90 
Table 22 - Plant 1 - Sub-model 1 - Transfer Function Numerator Parameters.

\begin{tabular}{|c|c|c|c|c|c|c|c|c|c|}
\hline$P I$ & $N$ & $u_{I}$ & $u_{2}$ & $u_{3}$ & $u_{4}$ & $u_{5}$ & $u_{6}$ & $u_{7}$ & $d_{l}$ \\
\hline \multirow{6}{*}{$y_{I}$} & $b 5$ & 0 & 0 & 0 & 0 & 0 & 0 & 0.0000827924 & 0 \\
\hline & $b 4$ & 0.0090046489 & 0 & -0.0000642898 & 0 & 0 & 0 & 0.0051925815 & 0 \\
\hline & $b 3$ & 0.0043974221 & 0 & -0.0970145448 & 0.0002514577 & 0 & 0.0112212326 & -0.0000020317 & 0.0006379532 \\
\hline & $b 2$ & 0.0005638163 & -0.6594430621 & 0.0354375930 & 0.1170434304 & 0.0099090955 & -0.0022919057 & -0.0039022526 & -0.0034168098 \\
\hline & bl & 0.0000061370 & 6.1537399176 & 0.0000181738 & 0.0314538294 & -0.0112287701 & -0.0056052935 & -0.0004885562 & -0.0000818437 \\
\hline & $b 0$ & 0.0000000934 & -0.1649113672 & 0.0000027346 & 0.0022399915 & -0.0007643234 & -0.0000044667 & -0.0000146137 & -0.0000082694 \\
\hline \multirow{6}{*}{$y_{2}$} & $b 5$ & 0 & 0 & 0 & 0 & 0 & 0 & 0 & -0.0039176576 \\
\hline & $b 4$ & 0.0747179135 & 0 & 0 & -0.0000711789 & 0 & 0 & 0 & 0.0442060078 \\
\hline & $b 3$ & 0.0816719547 & -0.0532769731 & 0 & 0.7364941116 & 0.0790217947 & 1.4750338736 & 4.6963905563 & -0.0282380084 \\
\hline & $b 2$ & 0.0078314266 & 0.7789631714 & 0.1158548845 & 0.8395632971 & -0.0331556249 & -0.2509076518 & -0.4239366006 & -0.0132062440 \\
\hline & $b 1$ & 0.0000783820 & 1.4515902382 & 0.1946674648 & 1.2313993214 & -0.0237464687 & -0.6868907059 & -2.7430205912 & -0.0003776090 \\
\hline & $b 0$ & 0.0000022620 & 0.0604834238 & 0.0225121073 & 0.1265831132 & -0.0010333426 & -0.0654041668 & -0.3381401040 & -0.0000307580 \\
\hline \multirow{6}{*}{$y_{3}$} & $b 5$ & 0 & 0 & 0 & 0 & 0.0006031578 & 0 & 0 & -0.0078241560 \\
\hline & $b 4$ & -0.0010643203 & 0 & 0 & 0 & 0.1770685145 & 0.2292102963 & 0 & 0.1387827555 \\
\hline & $b 3$ & -0.0451047972 & 0 & 0 & 0 & -0.0374658115 & -0.0462469651 & 1.3875247314 & -0.1056419195 \\
\hline & $b 2$ & 0.3155489702 & $0.800209834 I$ & 0 & 0 & -0.0873803269 & -0.1071820022 & -0.4942325967 & -0.0255144588 \\
\hline & $b 1$ & 0.3454852269 & 0.6807099310 & 1.6339371062 & 14.6682006006 & -0.0075465301 & -0.0088016359 & -0.6833511239 & -0.0008565464 \\
\hline & $b 0$ & 0.0268754518 & -0.0760942812 & 0.2388023195 & 2.0351357929 & -0.0000103318 & -0.0000021889 & -0.0490273716 & -0.0000573083 \\
\hline \multirow{6}{*}{$y_{4}$} & $b 5$ & 0 & 0 & 0 & 0 & 0 & 0 & 0.0004709939 & 0 \\
\hline & $b 4$ & -0.0162231038 & 0 & -0.2452035375 & 0.0004679805 & 0 & 0.1106209763 & 0.0752665477 & 0 \\
\hline & $b 3$ & 0.0978435571 & 0 & 0.0669171922 & 0.8430420219 & 0.3091892740 & -0.0038294545 & -0.0302638315 & 0.0259084502 \\
\hline & $b 2$ & 0.0093171805 & 0 & 0.1641553439 & 0.1979932971 & -0.2402945056 & -0.0758286215 & -0.0357991060 & -0.0638219403 \\
\hline & $b 1$ & 0.0001607394 & 5.0075273851 & 0.0258918941 & 0.0082076933 & -0.0863192117 & -0.0083275461 & -0.0015410310 & -0.0017041691 \\
\hline & $b 0$ & 0.0000148895 & -0.6795222504 & 0.0008431281 & 0.0000186757 & -0.0000986479 & -0.0000073962 & -0.0000315367 & -0.0001405162 \\
\hline \multirow{6}{*}{$y_{5}$} & $b 5$ & 0 & 0 & 0 & 0 & 0 & 0 & 0 & 0 \\
\hline & $b 4$ & 0 & 0 & 0 & 0 & 0 & 0 & 0 & 0 \\
\hline & $b 3$ & 0.0867369582 & 0 & 0 & 258.6169725597 & 0.0589507346 & 0.0683384722 & 0.0606853155 & 0.0324977233 \\
\hline & $b 2$ & 0.0108980333 & 0.2998447194 & 0 & 70.4315334882 & -0.0439624255 & -0.0598330588 & -0.0722236828 & -0.0373699023 \\
\hline & $b 1$ & 0.0001717923 & -0.0351875261 & -1.8879141230 & 0.1818354435 & -0.0087983807 & -0.0038011994 & 0.0001152627 & -0.0008893935 \\
\hline & $b 0$ & 0.0000210849 & -0.0014306767 & 2.3445408666 & 0.0064477173 & -0.0000150397 & -0.0000410719 & -0.0001240765 & -0.0000931558 \\
\hline \multirow{6}{*}{$y_{6}$} & $b 5$ & 0 & 0 & 0 & 0 & 0.0005307665 & 0 & 0 & -0.0029482526 \\
\hline & $b 4$ & 0.0821977635 & 0 & -0.0001504349 & 0 & 0.0955779821 & 0 & 0 & 0.0663042250 \\
\hline & $b 3$ & 0.0178273905 & 0.0381162756 & -0.0597518725 & 0 & -0.0242476520 & 0.7581758789 & 0.3249895310 & -0.0458074657 \\
\hline & $b 2$ & 0.0010297013 & 0.3497284145 & 0.0530862854 & 0 & -0.0390703616 & -0.4194856202 & -0.0815733115 & -0.0130573512 \\
\hline & $b 1$ & 0.0000176315 & 0.1129314912 & 0.0710194257 & 19.0693044826 & -0.0018749581 & -0.1995891094 & -0.1903307177 & -0.0004125399 \\
\hline & $b 0$ & 0.0000001491 & -0.0127890170 & 0.0053773140 & 2.7287280009 & -0.0000001333 & -0.0007969403 & -0.0140803468 & -0.0000279777 \\
\hline \multirow{6}{*}{$y_{7}$} & $b 5$ & 0 & 0 & 0 & 0 & 0 & 0 & 0 & 0 \\
\hline & $b 4$ & 0 & 0 & 0 & 0 & 0 & 0 & 0 & 0 \\
\hline & $b 3$ & 0 & 0 & -3.0271358881 & 0 & 0.3880124442 & 0.4163597840 & 0 & 0.2775690202 \\
\hline & $b 2$ & 0 & 0.1091487602 & -5.9243531239 & 4.9786105812 & 0.0181331491 & -0.0305611047 & 0.3260241842 & -0.0109702671 \\
\hline & $b 1$ & 0.1376325604 & 0.0658030622 & -2.1194935057 & 0.7924741674 & -0.0330846199 & -0.0362997601 & 0.0099098431 & 0.0003656075 \\
\hline & $b 0$ & 0.0255050957 & -0.0138732779 & -0.1342463138 & 0.0000251844 & -0.0009484470 & -0.0001324338 & -0.0184660586 & -0.0000559589 \\
\hline \multirow{6}{*}{$y_{8}$} & $b 5$ & -0.0121949090 & 0 & 0 & -0.0015066432 & 0 & 0 & 0 & 0 \\
\hline & $b 4$ & 0.0970648052 & 0 & 0 & 0.0000734494 & 0.0690251188 & 0 & 0 & 0 \\
\hline & $b 3$ & 0.0035051418 & 0 & -0.0042571857 & 0.0000724827 & -0.0652502557 & -0.0264413295 & -0.0170304497 & -0.0138963430 \\
\hline & $b 2$ & 0.0001970770 & 0 & 0.0044588544 & 0.0000020141 & -0.0021282216 & -0.0051758494 & -0.0019693558 & -0.0002502991 \\
\hline & $b 1$ & 0.0000063562 & 0.7102439397 & 0.0029591906 & 0.0000003236 & -0.0007543690 & -0.0000490512 & -0.0000455681 & -0.0000426759 \\
\hline & $b 0$ & 0.0000000052 & -0.1597287902 & 0.0022493446 & 0.0000000030 & -0.0000351613 & -0.0000094627 & -0.0000051709 & -0.0000007559 \\
\hline \multirow{6}{*}{$y_{9}$} & $b 5$ & 0 & 0 & -0.0000164821 & 0 & 0 & 0 & 0 & 0 \\
\hline & $b 4$ & 0 & 0 & -0.0010495568 & 0 & 0 & 0 & 0.0001548918 & 0 \\
\hline & $b 3$ & 0 & 0 & 0.0012967152 & 0.0000115087 & 0.0309192381 & 0.0471477828 & 0.0223837834 & 0 \\
\hline & $b 2$ & 0.0359652354 & 0.0671704385 & 0.0005591556 & 0.0000029886 & -0.0508373933 & -0.0700138198 & -0.0239104759 & -0.0050198665 \\
\hline & $b 1$ & 0.0276181022 & -0.0015624070 & 0.0000409578 & 0.0000000539 & -0.0085309103 & -0.0100855729 & -0.0035153836 & -0.0001168482 \\
\hline & $b 0$ & 0.0019580329 & -0.0006271116 & 0.0000001146 & 04 & -0.0000044584 & -0.0000020480 & -0.0000460903 & -0.0000124103 \\
\hline \multirow{6}{*}{$y_{10}$} & $b 5$ & 0.0056735964 & 0 & 0.0000138869 & -0.0000967890 & 0 & 0 & 0 & 0 \\
\hline & $b 4$ & 0.0410511767 & 0 & 0.0005097213 & 0.0004647256 & 0.0001841934 & 0 & 0.0002185129 & 0 \\
\hline & $b 3$ & 0.0295247698 & -0.0192206172 & 0.0000136878 & -0.0000080792 & 0.0347347974 & 0.4157241285 & 0.0321966102 & 0.0967770094 \\
\hline & $b_{2}$ & 0.0004325970 & 0.0498177801 & 0.0000020999 & 0.0000031314 & -0.0026645324 & -0.2338815381 & 0.0024139064 & -0.0431490673 \\
\hline & $b 1$ & 0.0000189232 & -0.0062600699 & 0.0000000387 & -0.0000000149 & -0.0141678732 & -0.0022116008 & -0.0110937285 & -0.0008018156 \\
\hline & $b 0$ & 0.0000002495 & -0.0012845818 & 06 & 0.0000000019 & -0.0004648858 & -0.0002996656 & -0.0005389941 & -0.0001150853 \\
\hline \multirow{6}{*}{$y_{H I}$} & $b 5$ & 0 & 0 & 0 & 0 & 0 & 0.0003436242 & 0 & 0 \\
\hline & $b 4$ & 0.0003965794 & 0 & 0 & 0.0000233070 & 0 & 0.0181322527 & 0.0137018772 & 0 \\
\hline & $b 3$ & 0.0337817002 & 0 & -0.0051585523 & -0.0006363237 & 0.0237010712 & -0.0077802518 & -0.0025838221 & 0 \\
\hline & $b 2$ & 0.0039805533 & 0 & 0.0060271977 & -0.0009181902 & -0.0149834729 & -0.0203592838 & -0.0176664947 & -0.0111466432 \\
\hline & $b 1$ & 0.0000548537 & 0.5327197971 & 0.0020309064 & -0.0003318486 & -0.0170900199 & -0.0000024080 & -0.0004839236 & -0.0002498950 \\
\hline & bo & 0.0000062141 & -0.1692145439 & 0.0020933222 & 0.0002137905 & -0.0000385986 & -0.0000014032 & -0.0000093485 & -0.0000258462 \\
\hline
\end{tabular}


Table 23 - Plant 1 - Sub-model 1 - Transfer Function Denominator Parameters.

\begin{tabular}{|c|c|c|c|c|c|c|c|c|c|}
\hline$P I$ & $D$ & $u_{1}$ & $u_{2}$ & $u_{3}$ & $u_{4}$ & $u_{5}$ & $u_{6}$ & $u_{7}$ & $d_{l}$ \\
\hline \multirow{6}{*}{$y_{I}$} & $a 5$ & 1 & 1 & 0 & 0 & 0 & 0 & 1 & 1 \\
\hline & a4 & 0.6376390753 & 57.1240909686 & 1 & 0 & 1 & I & 1.5122470740 & 0.8136305093 \\
\hline & $a 3$ & 0.1322298355 & 156.7735836415 & 8.1357214750 & 1 & 1.5801584466 & 1.7196928081 & 1.5074339043 & 1.3362904921 \\
\hline & $a_{2}$ & 0.0080263469 & 236.5827898243 & 0.9439806983 & 2.2100051025 & 3.0070639878 & 1.3360674485 & 0.3008729069 & 0.1622953301 \\
\hline & $a I$ & 0.0000950738 & 165.6208330689 & 0.0007965546 & 0.4713110802 & 0.5212112141 & 0.1228274930 & 0.0211192310 & 0.0067340413 \\
\hline & $a 0$ & 0.0000010704 & 15.4518103400 & 0.0000924136 & 0.0273070331 & 0.0201899285 & 0.0000599817 & 0.0004773942 & 0.0002897067 \\
\hline \multirow{6}{*}{$y_{2}$} & $a 5$ & 1 & 1 & 1 & 0 & 0 & 1 & 1 & 1 \\
\hline & a4 & 1.3278953420 & 3.6576647817 & 4.9012112744 & 1 & 1 & 16.6094350165 & 95.8401252103 & 1.8201627346 \\
\hline & $a 3$ & 0.3752173026 & 8.9158923567 & 7.9942874779 & 11.4994150299 & 1.8341648229 & 31.1553933763 & 168.6592112869 & 1.2230239712 \\
\hline & $a 2$ & 0.0202036315 & 9.8120268519 & 9.7774412065 & 13.9017159872 & 1.4103307078 & 29.0997296260 & 171.6892181336 & 0.1433695161 \\
\hline & $a l$ & 0.0002547788 & 6.5966726403 & 3.5632778260 & 15.5351561628 & 0.2035035379 & 6.2701698644 & 44.2651784920 & 0.0059863981 \\
\hline & $a 0$ & 0.0000051845 & 0.7428053223 & 0.2723426722 & 1.3937089513 & 0.0063406477 & 0.3219933993 & 2.6568020301 & 0.0002481811 \\
\hline \multirow{6}{*}{$y_{3}$} & $a 5$ & 0 & 1 & I & 0 & 1 & I & 1 & 1 \\
\hline & a4 & 1 & 2.4245475283 & 5.2755653801 & 1 & 1.9457762481 & 1.9961759251 & 11.7769250694 & 2.1087801542 \\
\hline & $a 3$ & 2.0028746433 & 5.1984982118 & 11.5445251418 & 5.6238719390 & 1.7586052625 & 1.7818732706 & 20.9001916538 & 1.2119772528 \\
\hline & $a 2$ & 2.7893292198 & 4.8294145910 & 17.1270741318 & 30.7544473828 & 0.4571749186 & 0.4526660670 & 17.5715500180 & 0.1446742887 \\
\hline & $a l$ & 0.8630318094 & 2.8794271345 & 7.0067920429 & 46.6570644154 & 0.0264278913 & 0.0249497504 & 4.2001278868 & 0.0060438319 \\
\hline & $a 0$ & 0.0475172625 & 0.4609213712 & 0.6115198372 & 5.2150081579 & 0.0000336321 & 0.0000047723 & 0.2119332424 & 0.0002584772 \\
\hline \multirow{6}{*}{$y_{4}$} & $a 5$ & 0 & 1 & $I$ & 0 & 1 & 1 & 1 & 1 \\
\hline & $a 4$ & 1 & 7.5881091615 & 2.9559319124 & 1 & 3.7898928598 & 1.7658249566 & 1.5997777071 & 1.0649173198 \\
\hline & $a 3$ & 0.2635317565 & 21.8722984645 & 2.4740266887 & 2.1938582561 & 6.0117423820 & 1.5581603359 & 1.1360377891 & 1.9079339328 \\
\hline & $a 2$ & 0.0148306566 & 26.1850741805 & 0.6039037884 & 0.3563116655 & 2.6625149784 & 0.3301554825 & 0.1637285866 & 0.2958316331 \\
\hline & al & 0.0004260715 & 19.2491806619 & 0.0516786169 & 0.0127994659 & 0.2295704910 & 0.0180718191 & 0.0058910766 & 0.0112649004 \\
\hline & $a 0$ & 0.0000213632 & 2.1338495124 & 0.0013213614 & 0.0000304089 & 0.0002185642 & 0.0000145557 & 0.0001031183 & 0.0004790850 \\
\hline \multirow{6}{*}{$y_{5}$} & a5 & 1 & 1 & 0 & 1 & 0 & 0 & $l$ & 0 \\
\hline & a4 & 1.6580025362 & 2.0006753461 & 1 & 457.0479648004 & 1 & 1 & 1.8909971144 & 1 \\
\hline & $a 3$ & 0.3330722403 & 2.7495806366 & 8.1534543966 & 1075.9588635942 & 1.6878895950 & 1.6286853258 & 2.6566233896 & 1.5869053470 \\
\hline & $a 2$ & 0.0194010926 & 1.4793237172 & 21.8234721865 & 98.8421596462 & 0.4024010878 & 0.2329158275 & 0.2373771443 & 0.1789559326 \\
\hline & al & 0.0006438550 & 0.1553686195 & 35.9909918166 & 0.3283061034 & 0.0234793039 & 0.0092526531 & 0.0045494059 & 0.0076433876 \\
\hline & $a 0$ & 0.0000314555 & 0.0035939288 & 3.3698219597 & 0.0087679585 & 0.0000375938 & 0.0000873961 & 0.0004012124 & 0.0003145127 \\
\hline \multirow{6}{*}{$y_{6}$} & a5 & 1 & 1 & 0 & 0 & 1 & 1 & 1 & 1 \\
\hline & $a 4$ & 0.6140711714 & 2.1960723463 & 1 & 1 & 2.0352841347 & 7.5141247204 & 5.9853857184 & 2.0737858045 \\
\hline & $a 3$ & 0.0976140892 & 3.9822238468 & 2.4681022507 & 9.1676510426 & 1.8353589886 & 13.8377073705 & 11.9193206955 & 1.3893296425 \\
\hline & $a 2$ & 0.0047715333 & 3.3912359210 & 2.7313277561 & 83.8700654331 & 0.4472053389 & 9.8533544748 & 10.5147557568 & 0.1919253203 \\
\hline & al & 0.0000823666 & 1.5590911264 & 0.8274170919 & 131.6245735233 & 0.0170416048 & 1.5246720417 & 2.9770516802 & 0.0074797246 \\
\hline & $a 0$ & 0.0000005926 & 0.2284077604 & 0.0484101856 & 15.9715960487 & 0.0000026470 & 0.0054389259 & 0.1609326046 & 0.0003336819 \\
\hline \multirow{6}{*}{$y_{7}$} & $a 5$ & 0 & $I$ & $I$ & 1 & 1 & 1 & 0 & 1 \\
\hline & a4 & 1 & 1.8148804636 & 6.0536403540 & 6.4241505644 & 5.3113139195 & 5.0512876743 & 1 & 2.0608536674 \\
\hline & $a 3$ & 2.1020247489 & 3.8583457155 & 18.4650334338 & 36.0886567056 & 10.3013530737 & 9.6607909649 & 4.7869368711 & 6.8582307181 \\
\hline & $a_{2}$ & 4.3823966491 & 2.9580136241 & 21.6225906701 & 56.6163820428 & 6.3610635551 & 5.3827757642 & 9.3967792883 & 1.3362625300 \\
\hline & al & 2.2205404909 & 1.6256626938 & 7.8854340393 & 5.8856372886 & 0.8128597136 & 0.5406899398 & 5.1618982900 & 0.0516441851 \\
\hline & $a 0$ & 0.1936483460 & 0.1839608115 & 0.6364571386 & 0.0002308046 & 0.0176920243 & 0.0017990754 & 0.4815347622 & 0.0025668940 \\
\hline \multirow{6}{*}{$y_{8}$} & a5 & 1 & 1 & 0 & 1 & 1 & $I$ & 1 & 1 \\
\hline & a4 & 0.1503165495 & 5.2914989943 & 1 & 0.0823363411 & 1.7612233230 & 0.7143893521 & 0.7118270698 & 0.6364857458 \\
\hline & $a 3$ & 0.0060416343 & 9.4383170595 & 1.6288532678 & 0.0186780306 & 0.2808403785 & 0.1869629710 & 0.1427399489 & 0.0773446327 \\
\hline & $a 2$ & 0.0003013495 & 12.1910527159 & 1.9415184315 & 0.0002493173 & 0.0284959740 & 0.0126794867 & 0.0091525279 & 0.0029083717 \\
\hline & $a l$ & 0.0000071973 & 7.0272021743 & 1.0849974820 & 0.0000465862 & 0.0030861613 & 0.0003410102 & 0.0003667913 & 0.0002271625 \\
\hline & $a 0$ & 0.0000000114 & 0.6875865416 & 0.0913812231 & 0.0000000154 & 0.0000952984 & 0.0000209327 & 0.0000190806 & 0.0000029419 \\
\hline \multirow{6}{*}{$y_{9}$} & $a 5$ & 0 & 1 & $I$ & 1 & 1 & 1 & 0 & 0 \\
\hline & $a 4$ & 0 & 2.6709005522 & 0.9036684355 & 0.2617045411 & 2.9727260851 & 2.9932566279 & 1 & 0 \\
\hline & $a 3$ & 1 & 4.1595781982 & 2.0342735754 & 0.0489651777 & 6.7243287020 & 7.0669115231 & 4.0633355224 & 1 \\
\hline & $a 2$ & 1.1685556864 & 3.4141065060 & 0.2971676921 & 0.0032226781 & 1.8625722509 & 1.8595146699 & 1.0704070385 & 0.0958438593 \\
\hline & al & 0.1850699637 & 0.6893360445 & 0.0116842004 & 0.0000348537 & 0.0859687417 & 0.0783596957 & 0.0565865968 & 0.0046538370 \\
\hline & $a 0$ & 0.0060289535 & 0.0266493390 & 0.0000246267 & 0.0000002589 & 0 & 0.0000201669 & 0.0006022054 & 0.0001558030 \\
\hline \multirow{6}{*}{$y_{10}$} & a5 & 1 & 0 & 1 & 1 & 0 & 0 & 0 & 1 \\
\hline & a4 & 0.9306103428 & 1 & 0.1063412273 & 0.0267282066 & 1 & $I$ & 1 & 3.3101140498 \\
\hline & $a 3$ & 0.1221665947 & 1.6811218332 & 0.0078952170 & 0.0066844438 & 2.2590439479 & 16.9902911759 & 2.4091250004 & 7.3266801993 \\
\hline & $a 2$ & 0.0012536788 & 1.9082785185 & 0.0003241362 & 0.0000928110 & 1.7318674230 & 2.4789318458 & 1.8934078219 & 0.9991431874 \\
\hline & $a I$ & 0.0000836952 & 0.4641571252 & 0.0000114926 & 0.0000092154 & 0.2176257211 & 0.0454442316 & 0.2651306812 & 0.0420559587 \\
\hline & $a 0$ & 0.0000004220 & 0.0259298442 & 0 & 0.0000000334 & 0.0050433158 & 0.0027168395 & 0.0080547305 & 0.0017131294 \\
\hline \multirow{6}{*}{$y_{I I}$} & $a 5$ & 0 & 0 & 1 & 0 & 0 & 1 & 1 & 0 \\
\hline & $a 4$ & 1 & 1 & 4.0983180413 & 1 & 1 & 1.8742551779 & 2.6825700945 & 1 \\
\hline & $a 3$ & 0.3106013697 & 19.3228011827 & 8.7774769532 & 1.0770241763 & 3.0245839487 & 1.8409289498 & 2.5703082765 & 1.6621158799 \\
\hline & $a 2$ & 0.0224269003 & 24.2754485938 & 9.0843086864 & 2.7237344215 & 2.0572526620 & 0.2449227627 & 0.4299471920 & 0.2816174992 \\
\hline & $a l$ & 0.0004886125 & 26.2075178899 & 6.1064968801 & 1.1386682736 & 0.2700825364 & 0.0001327012 & 0.0111093562 & 0.0102825282 \\
\hline & $a 0$ & 0.0000328028 & 4.9283050708 & 0.7390441708 & 0.1384226495 & 0.0005657081 & 0.0000175345 & 0.0001905284 & 0.0005086611 \\
\hline
\end{tabular}


Table 24 - Plant 1 - Sub-model 2 - Transfer Function Numerator Parameters.

\begin{tabular}{|c|c|c|c|c|c|c|c|c|c|}
\hline$P I$ & $N$ & $u_{1}$ & $u_{2}$ & $u_{3}$ & $u_{4}$ & $u_{5}$ & $u_{6}$ & $u_{7}$ & $d_{l}$ \\
\hline \multirow{6}{*}{$y_{l}$} & $b 5$ & 0 & 0.0002146302 & 0 & 0 & 0.0001991927 & 0 & 0 & 0 \\
\hline & $b 4$ & 0 & -0.0091778720 & 0 & -0.0877340801 & 0.0030313393 & 0.0022730715 & 0.0012874287 & 0.0029588065 \\
\hline & $b 3$ & -0.0079423550 & 0.0181795846 & -0.0002508646 & -0.0435629399 & -0.0018012112 & -0.0016158390 & -0.0010001109 & -0.0000777862 \\
\hline & $b 2$ & 0.0095037786 & -0.0008025337 & -0.0249249493 & -0.0046837892 & 0.0000052602 & 0.0000215192 & -0.0000351598 & -0.0015015828 \\
\hline & $b l$ & 0.0000194712 & 0.0000091555 & -0.0003267190 & -0.0000143808 & -0.0000048748 & -0.0000043698 & -0.0000013400 & 0.0000000542 \\
\hline & bo & 0.0000007962 & -0.0000005514 & 0.0006744252 & -0.0000005031 & -0.0000000094 & 0.0000000167 & -0.0000000534 & -0.0000022689 \\
\hline \multirow{6}{*}{$y_{2}$} & $b 5$ & 0 & 0 & 0 & 0 & 0 & 0 & 0 & 0 \\
\hline & $b 4$ & 0.0521636527 & 0 & -0.0001009798 & 0 & 0.0003659946 & 0.0004327485 & 0 & 0.0007363627 \\
\hline & $b 3$ & 0.0060836313 & 0 & -0.0065319674 & 0 & 0.0434373753 & 0.0594636373 & 29.9651601030 & 0.0362862506 \\
\hline & $b 2$ & 0.0005396908 & 0.3349364887 & 0.0171041502 & 0 & -0.0110023791 & -0.0140148487 & 7.2259065302 & -0.0132590808 \\
\hline & $b l$ & 0.0000033853 & 0.5725661320 & 0.0244778080 & -18.5684789504 & -0.0195015508 & -0.0243091461 & -26.7994206729 & -0.0164533929 \\
\hline & $b 0$ & 0.0000003148 & 0.0282268255 & 0.0024515306 & -1.3873768420 & -0.0021226183 & -0.0026178983 & -4.4093097111 & -0.0015633454 \\
\hline \multirow{6}{*}{$y_{3}$} & $b 5$ & -0.0337646192 & 0 & 0 & 0 & 0.0006279500 & 0.0007721963 & 0.0007758228 & 0 \\
\hline & $b 4$ & 0.2363204459 & 0 & 0 & 0 & 0.2172214216 & 0.2808631326 & 0.1634205421 & 0.0030942392 \\
\hline & $b 3$ & 0.0225452558 & 0.2010943643 & 0 & -0.0036898623 & -0.0501463474 & -0.0641572368 & -0.0439888308 & 0.1756340215 \\
\hline & $b 2$ & 0.0016099874 & 0.4804647470 & 0 & -0.7247103167 & -0.1083237102 & -0.1312876888 & -0.0853293027 & -0.0662359789 \\
\hline & $b l$ & 0.0000029123 & 0.0068414809 & 1.7897630169 & -0.2543273379 & -0.0107644133 & -0.0132143858 & -0.0081850536 & -0.0967420312 \\
\hline & bo & 0.0000001300 & -0.0069024056 & 0.2719221215 & -0.0166320858 & -0.0002140996 & -0.0002729038 & -0.0001252842 & -0.0078371380 \\
\hline \multirow{6}{*}{$y_{4}$} & $b 5$ & 0 & 0 & 0 & 0 & 0 & 0 & 0 & 0 \\
\hline & $b 4$ & 0 & -0.1336041481 & 0 & 0 & 0 & 0 & -0.0000959287 & 0.1023303000 \\
\hline & $b 3$ & 0.1414380112 & 0.3603402729 & -0.6030535362 & 0 & 0.1277869614 & 0.1623408600 & 0.0966623292 & -0.0289608721 \\
\hline & $b 2$ & 0.0580529255 & -0.0268997006 & 0.6295819702 & 0 & -0.0727991469 & -0.0899402405 & -0.0585538252 & -0.0706419973 \\
\hline & $b l$ & 0.0010176285 & -0.0030226315 & 0.1416424460 & -24.0670993774 & -0.0437659767 & -0.0519242744 & -0.0355953678 & -0.0086890873 \\
\hline & $b 0$ & 0.0000033161 & -0.0001232224 & 0.0002680490 & -3.9779829636 & -0.0007629059 & -0.0007614144 & -0.0006209541 & -0.0001929708 \\
\hline \multirow{6}{*}{$y_{5}$} & $b 5$ & 0 & 0 & 0 & 0 & 0 & -0.0003280257 & 0 & 0 \\
\hline & $b 4$ & 0 & 0.0046948424 & 0 & 0 & 0.0375018471 & 0.0929178364 & 0.0280719387 & 0 \\
\hline & $b 3$ & 0.0707807272 & -0.1129361573 & 0 & 0 & 0.0269282941 & -0.0829216990 & 0.0164128009 & 0.0670042328 \\
\hline & $b 2$ & 0.0022775607 & 0.2684966859 & 0 & -2.0016005150 & -0.0634511952 & 0.0002001833 & -0.0486720999 & -0.0983470215 \\
\hline & $b l$ & 0.0001089771 & -0.0355565109 & -1.6060185179 & -0.8949813679 & -0.0000028644 & -0.0002049021 & -0.0000000700 & -0.0039557350 \\
\hline & bo & 0.0000032533 & -0.0010936047 & 1.9607818990 & -0.0913878226 & -0.0000025468 & -0.0000001159 & -0.0000067180 & -0.0001594683 \\
\hline \multirow{6}{*}{$y_{6}$} & $b 5$ & 0 & 0 & 0 & 0 & 0 & 0.0004505751 & 0 & 0.0014077985 \\
\hline & $b 4$ & 0.0858024497 & 0 & -0.0608132462 & 0.0000268360 & 0.1067491631 & 0.1456024414 & 0.0777383310 & 0.0874905741 \\
\hline & $b 3$ & 0.1492274059 & 0 & 0.0469674439 & -0.3840213399 & -0.0307634743 & -0.0464665621 & -0.0253199179 & -0.0306304452 \\
\hline & $b 2$ & 0.0161710214 & 0.3157452976 & 0.0710748110 & -0.6460479300 & -0.0445654917 & -0.0496766341 & -0.0345228444 & -0.0432067962 \\
\hline & $b l$ & 0.0004277372 & 0.5487360624 & 0.0050956458 & -1.0102261358 & -0.0029798129 & -0.0048663910 & -0.0022543584 & -0.0035318038 \\
\hline & bo & 0.0000004574 & -0.0575535158 & 0.0000052445 & -0.1210646585 & 0.0000000785 & -0.0001584398 & 0.0000000192 & -0.0000391020 \\
\hline \multirow{6}{*}{$y_{7}$} & $b 5$ & -0.0083946917 & 0 & 0 & 0 & 0 & 0.0008093867 & 0 & 0 \\
\hline & $b 4$ & 0.0401243332 & -0.0592688987 & 0 & 0 & 0 & 0.1136692777 & 0 & 0 \\
\hline & $b 3$ & 0.0010344802 & 0.0820757314 & -314.9523250879 & 0 & 0 & -0.0076795437 & 0 & 0.3852055680 \\
\hline & $b 2$ & 0.0000815129 & 0.0693023977 & -1111.4994997105 & 0 & 0.3800259882 & -0.0099875009 & 0.3384561951 & 0.2106610259 \\
\hline & $b l$ & 0.0000021456 & -0.0137862773 & -384.2158446832 & -29.6418815565 & -0.0126609687 & -0.0000001350 & 0.0101257614 & -0.0451727921 \\
\hline & $b 0$ & -0.0000000014 & -0.0002087618 & -23.2691026127 & -4.4540756355 & -0.0317558105 & -0.0000002988 & -0.0231595213 & -0.0053058495 \\
\hline \multirow{6}{*}{$y_{8}$} & $b 5$ & 0 & 0 & 0 & -0.0000323204 & 0 & 0.0005355930 & 0 & 0 \\
\hline & $b 4$ & 0.0063559044 & -0.0074842651 & 0 & 0.0079870465 & 0.0107381233 & 0.0113991776 & 0 & 0 \\
\hline & $b 3$ & 0.0684302768 & 0.0198918749 & 0.0003903671 & -0.0006060402 & -0.0127017484 & -0.0134820848 & 0 & -0.0014367760 \\
\hline & $b 2$ & 0.0098427817 & -0.0049155320 & 0.0004873674 & -0.0005184105 & -0.0001956947 & -0.0007796724 & 0.0110529353 & -0.0087370890 \\
\hline & $b 1$ & 0.0003623304 & 0.0000424227 & 0.0000219739 & -0.0000189613 & -0.0000134171 & -0.0000199797 & -0.0196982484 & -0.0000758577 \\
\hline & bo & 0.0000010432 & -0.0000156402 & 0.0000084517 & -0.0000054193 & -0.0000000739 & -0.0000012031 & -0.0020074994 & -0.0000010334 \\
\hline \multirow{6}{*}{$y_{9}$} & $b 5$ & -0.0130697290 & 0 & 0 & 0 & 0 & 0 & 0 & 0 \\
\hline & $b 4$ & 0.0851551426 & -0.0146580685 & -0.0031936663 & 0 & 0 & 0 & 0 & 0 \\
\hline & $b 3$ & 0.0028532439 & 0.0563754993 & 0.0036821408 & 0 & 0 & -0.0219922201 & 0 & 0 \\
\hline & $b 2$ & 0.0001502676 & -0.0073639632 & 0.0011757357 & 0.0004164096 & 0 & -0.0002864484 & -0.0237533887 & -0.0299635544 \\
\hline & $b 1$ & 0.0000055956 & 0.0000929425 & 0.0000040912 & -0.0002031343 & -0.5706961589 & -0.0000154155 & -0.0000004559 & -0.0038388194 \\
\hline & bo & 0.0000000119 & -0.0000293714 & -0.0000000367 & -0.0000001145 & -0.0671957832 & -0.0000000917 & -0.0000250836 & -0.0001096460 \\
\hline \multirow{6}{*}{$y_{I 0}$} & $b 5$ & -0.0060572555 & 0 & 0 & 0 & 0 & 0.0000737797 & 0 & 0 \\
\hline & $b 4$ & 0.0437713712 & -0.0088165804 & 0 & 0 & 0.0002127268 & 0.0427194468 & 0.0000927648 & 0 \\
\hline & $b 3$ & 0.0318135077 & 0.0319785020 & 0 & 0.0034857104 & 0.0443641067 & 0.0021497875 & 0.0368548069 & 0.0326595181 \\
\hline & $b 2$ & 0.0022059135 & -0.0081458033 & 0 & -0.0012411413 & -0.0071797241 & -0.0261361530 & -0.0027459713 & -0.0177493951 \\
\hline & $b l$ & 0.0000054979 & -0.0001382865 & 0.0010170757 & -0.0000307257 & -0.0148612980 & -0.0013902795 & -0.0098143257 & -0.0056016050 \\
\hline & $b 0$ & 0.0000002837 & -0.0000386645 & 0.0000013186 & -02 & -0.0009267309 & -0.0000194948 & -0.0005826612 & -0.0003276632 \\
\hline \multirow{6}{*}{$y^{\prime \prime}$} & $b 5$ & 0 & 0.0003831918 & -0.0000330637 & 0 & 0 & 0 & 0.0002230434 & 0 \\
\hline & $b 4$ & 0.1040332878 & -0.0247073304 & -0.0061841163 & 0 & 0.0383432016 & 0.0514251280 & 0.0269388996 & 0 \\
\hline & b3 & 0.0021919315 & 0.0822546796 & 0.0044392204 & 0.0135463363 & -0.0288359031 & -0.0381738374 & -0.0201305491 & 0 \\
\hline & $b 2$ & 0.0002618435 & -0.0284917187 & 0.0017175958 & 0.0019412132 & -0.0496629748 & -0.0649313674 & -0.0364963404 & 0 \\
\hline & $b l$ & 0.0000047342 & 0.0001897244 & 0.0015459534 & 0.0027038105 & -0.0024824425 & -0.0039339645 & -0.0016246151 & -0.2499667813 \\
\hline & bo & 0.0000000154 & -0.0000704215 & 0.0000501103 & -0.0030142777 & -0.0000487449 & -0.0000947204 & -0.0000284683 & -0.0188611926 \\
\hline
\end{tabular}


Table 25 - Plant 1 - Sub-model 2 - Transfer Function Denominator Parameters.

\begin{tabular}{|c|c|c|c|c|c|c|c|c|c|}
\hline$P I$ & $D$ & $u_{1}$ & $u_{2}$ & $u_{3}$ & $u_{4}$ & $u_{5}$ & $u_{6}$ & $u_{7}$ & $d_{l}$ \\
\hline \multirow{6}{*}{$y_{I}$} & $a 5$ & 0 & 1 & 0 & 1 & 1 & 1 & 0 & 1 \\
\hline & a4 & 1 & 1.5863431790 & 0 & 2.1803382245 & 1.1653529350 & 0.9127108126 & 1 & 3.1465133506 \\
\hline & $a 3$ & 1.9634013903 & 1.0929610333 & 1 & 0.8550422205 & 0.1062679781 & 0.0644747018 & 0.0818647411 & 1.3281985360 \\
\hline & $a_{2}$ & 0.2262916483 & 0.0733621716 & 2.2108900231 & 0.0678752044 & 0.0032936354 & 0.0026376131 & 0.0053487386 & 0.1066712159 \\
\hline & $a I$ & 0.0000817782 & 0.0014374533 & 0.5148179606 & 0.0002395325 & 0.0002699555 & 0.0001767125 & 0.0001043429 & 0.0019922814 \\
\hline & $a 0$ & 0.0000094198 & 0.0000325355 & 0.0331597387 & 0.0000068608 & 0.0000006742 & 0.0000000556 & 0.0000047653 & 0.0001530909 \\
\hline \multirow{6}{*}{$y_{2}$} & $a 5$ & 1 & 1 & 0 & 1 & 0 & 0 & 1 & 0 \\
\hline & a4 & 0.3586605734 & 3.1435165448 & 1 & 18.1643362325 & 1 & 1 & 1087.4648679863 & 1 \\
\hline & $a 3$ & 0.0347112457 & 7.2263765857 & 1.6189296032 & 76.2075014748 & 1.9132921918 & 2.0312439336 & 2154.7558528451 & 1.9421480424 \\
\hline & $a 2$ & 0.0020026558 & 7.4947368044 & 2.1229981129 & 304.6166419178 & 1.7759833082 & 1.8457684467 & 2572.4713057740 & 1.7558052732 \\
\hline & $a l$ & 0.0000207490 & 4.8353542906 & 0.7153229996 & 388.3135855956 & 0.4209199792 & 0.4262556543 & 888.7696869794 & 0.3759866373 \\
\hline & $a 0$ & 0.0000010856 & 0.5641451307 & 0.0503075212 & 25.7746010743 & 0.0244031913 & 0.0243706714 & 64.0157601313 & 0.0199645020 \\
\hline \multirow{6}{*}{$y_{3}$} & $a 5$ & 1 & 1 & 1 & 0 & 1 & 1 & 1 & 0 \\
\hline & $a 4$ & 0.3991593691 & 1.7094392482 & 5.4486123664 & 0 & 2.0415018007 & 2.1085185438 & 1.9612797413 & 1 \\
\hline & $a 3$ & 0.0339379718 & 2.5318482096 & 11.4383418668 & 1 & 1.8850142499 & 1.9142321211 & 1.8118449836 & 2.0303986971 \\
\hline & $a 2$ & 0.0018764020 & 1.6801529425 & 16.7909812311 & 2.2401194239 & 0.5085058024 & 0.5099233388 & 0.4876968877 & 1.8676529341 \\
\hline & $a l$ & 0.0000033498 & 0.4850132799 & 6.9549123331 & 0.6389431682 & 0.0358021003 & 0.0362296041 & 0.0329505560 & 0.4723729921 \\
\hline & $a 0$ & 0.0000001762 & 0.0349938839 & 0.6188665237 & 0.0378474662 & 0.0006410903 & 0.0006694947 & 0.0004659655 & 0.0259577782 \\
\hline \multirow{6}{*}{$y_{4}$} & $a 5$ & 0 & 1 & 1 & 0 & 0 & 0 & 0 & 1 \\
\hline & $a 4$ & 1 & 1.2951765223 & 3.2520621379 & 1 & 1 & 1 & 1 & 1.9659315548 \\
\hline & $a 3$ & 0.5326458606 & 1.2432125924 & 6.8929852163 & 6.0586652722 & 1.7125811687 & 1.7512344638 & 1.6462184814 & 1.6455653465 \\
\hline & $a 2$ & 0.0585181001 & 0.2220385226 & 2.3841822849 & 33.0046149577 & 0.9902837377 & 0.9841650722 & 0.9763860107 & 0.3594607856 \\
\hline & al & 0.0008872903 & 0.0125548069 & 0.1901982596 & 50.9921842281 & 0.1098362138 & 0.1053159357 & 0.1097852944 & 0.0251860816 \\
\hline & $a 0$ & 0.0000025378 & 0.0003109919 & 0.0003575290 & 5.2604727386 & 0.0016378032 & 0.0013455154 & 0.0016415341 & 0.0004649292 \\
\hline \multirow{6}{*}{$y_{5}$} & $a 5$ & 1 & 0 & 0 & 0 & 1 & 1 & $l$ & $I$ \\
\hline & $a 4$ & 0.7041379740 & 1 & 1 & 1 & 1.8129458714 & 1.6775030674 & 1.6906030371 & 2.3236528497 \\
\hline & $a 3$ & 0.0986204748 & 1.6580126375 & 4.3884671837 & 3.6041274084 & 1.6356099496 & 0.1512081004 & 1.5311969514 & 2.9430452663 \\
\hline & $a 2$ & 0.0028502793 & 1.0604933480 & 15.9254022438 & 8.1144306105 & 0.1370645541 & 0.0042303887 & 0.1292581932 & 0.3608217346 \\
\hline & al & 0.0001423993 & 0.1120001902 & 25.0038678638 & 1.8388353645 & 0.0000687994 & 0.0003671955 & 0.0002127275 & 0.0142521013 \\
\hline & $a 0$ & 0.0000026649 & 0.0021941299 & 2.3630123503 & 0.1076137288 & 0.0000056511 & 0.0000002200 & 0.0000178802 & 0.0003829989 \\
\hline \multirow{6}{*}{$y_{6}$} & $a 5$ & 1 & 1 & 1 & 0 & 1 & 1 & 1 & 1 \\
\hline & $a 4$ & 1.7514711252 & 3.0623146116 & 2.3226288187 & 1 & 2.1744554220 & 2.3064448515 & 2.0455218086 & 2.2278636863 \\
\hline & $a 3$ & 0.6583751275 & 6.5563052108 & 2.6612791463 & 4.2763828835 & 1.9851500854 & 2.0078676081 & 1.8833286610 & 2.1097605721 \\
\hline & $a 2$ & 0.0555031246 & 7.0386792863 & 0.8124032351 & 7.0193830250 & 0.5122965154 & 0.5166456043 & 0.4898531725 & 0.5731863595 \\
\hline & al & 0.0012953632 & 4.6306050281 & 0.0453345896 & 6.5329211718 & 0.0256250119 & 0.0397586735 & 0.0242208776 & 0.0352600767 \\
\hline & $a 0$ & 0.0000010900 & 0.9606460068 & 0.0000503355 & 0.6940991158 & 0 & 0.0011135936 & 0.0000003587 & 0.0003702289 \\
\hline \multirow{6}{*}{$y_{7}$} & $\overline{a 5}$ & 1 & 1 & 1 & 1 & 0 & 1 & 0 & 1 \\
\hline & a4 & 0.2512475731 & 2.4705772400 & 406.0004562291 & 10.8584288100 & 1 & 2.0690613263 & 1 & 4.6940318138 \\
\hline & $a 3$ & 0.0071567393 & 2.4556371630 & 1961.7520011917 & 60.8840037455 & 4.6546437534 & 1.2860244374 & 4.5820985625 & 11.1476292586 \\
\hline & $a_{2}$ & 0.0005175063 & 1.5189765894 & 3677.0068254992 & 239.6080670394 & 9.1526816090 & 0.1280276754 & 9.0889078817 & 10.4580667082 \\
\hline & al & 0.0000103205 & 0.1974073983 & 1399.2763018625 & 337.6633778572 & 5.3326736457 & 0.0000399174 & 5.3367394071 & 2.2731617217 \\
\hline & $a 0$ & 0.0000000134 & 0.0024908786 & 111.8001507921 & 32.6985057066 & 0.5266523888 & 0.0000039719 & 0.5224473956 & 0.1144739578 \\
\hline \multirow{6}{*}{$y_{8}$} & a5 & 1 & 1 & 1 & 1 & 1 & 1 & 0 & 1 \\
\hline & a4 & 3.0504392263 & 1.3903808122 & 0.5582292966 & 29.0988633487 & 1.9834821136 & 1.8095909167 & 1 & 1.7812822383 \\
\hline & $a 3$ & 0.7315342305 & 1.0577258493 & 1.2018544174 & 6.9624012105 & 0.2571980245 & 0.2945232188 & 3.4656779367 & 1.5055856751 \\
\hline & $a 2$ & 0.0604398121 & 0.1210196845 & 0.1666989405 & 0.8278837601 & 0.0056938940 & 0.0137248879 & 4.3616914398 & 0.1692941205 \\
\hline & $a l$ & 0.0016968156 & 0.0038189527 & 0.0233805869 & 0.0682349377 & 0.0002428236 & 0.0004345511 & 0.8835584044 & 0.0013953628 \\
\hline & a0 & 0.0000032047 & 0.0003553788 & 0.0021261344 & 0.0039630920 & 0.0000013145 & 0.0000163452 & 0.0438712875 & 0.0000203861 \\
\hline \multirow{6}{*}{$y_{9}$} & $a 5$ & 1 & 1 & 1 & 0 & 1 & 0 & 1 & 0 \\
\hline & $a 4$ & 0.1690003618 & 1.0654944405 & 0.9340274267 & 0 & 12.4756378719 & 1 & 0.8049609463 & 1 \\
\hline & $a 3$ & 0.0052687202 & 1.4208060977 & 2.0942286299 & 0 & 22.1117468233 & 0.0905405756 & 1.8674606401 & 1.7456078154 \\
\hline & $a 2$ & 0.0002969044 & 0.1658473654 & 0.1612055062 & 1 & 32.4322828994 & 0.0014365377 & 0.1482006585 & 0.3868629456 \\
\hline & al & 0.0000059368 & 0.0074166788 & 0.0003490659 & 0.0813347336 & 6.9020534078 & 0.0000583804 & 0.0019038054 & 0.0239826861 \\
\hline & $a 0$ & 0.0000000342 & 0.0004923439 & 0.0000032125 & 0 & 0.2817842012 & 0.0000001976 & 0.0001502978 & 0.0004738553 \\
\hline \multirow{6}{*}{$y_{10}$} & a5 & 1 & 1 & 0 & 0 & 0 & 1 & 0 & 0 \\
\hline & a4 & 0.9069574187 & 1.2500616859 & 1 & 1 & $I$ & 2.8851670571 & 1 & 1 \\
\hline & $a 3$ & 0.1474422506 & 1.5191853715 & 0.3602428086 & 5.7345674487 & 3.1613973878 & 2.4869349356 & 2.9291231117 & 2.8254065465 \\
\hline & $a 2$ & 0.0064791250 & 0.2527339655 & 2.0173064550 & 1.0883232686 & 1.9914664151 & 0.3858883536 & 1.8231944977 & 0.9882077373 \\
\hline & $a I$ & 0.0000226059 & 0.0125770621 & 0.2526402824 & 0.0152910706 & 0.3000167079 & 0.0143891464 & 0.2656463577 & 0.1143734343 \\
\hline & $a 0$ & 0.0000009730 & 0.0008372243 & 0 & 0.0000988037 & 0.0107585464 & 0.0001795438 & 0.0091519719 & 0.0039596981 \\
\hline \multirow{6}{*}{$y_{H I}$} & $a 5$ & 1 & 1 & 1 & 1 & 1 & 1 & 1 & 0 \\
\hline & a4 & 0.1689933991 & 1.3620224712 & 2.0773725498 & 4.7333583211 & 2.5330580504 & 2.6729051613 & 2.3452855084 & 1 \\
\hline & $a 3$ & 0.0056213301 & 1.6458034930 & 2.7334846259 & 9.6850145044 & 2.2498199207 & 2.3448082179 & 2.1443793560 & 7.0162296952 \\
\hline & $a 2$ & 0.0004205433 & 0.3138127425 & 1.7461871920 & 10.6349105993 & 0.3850463087 & 0.4196815266 & 0.3602769951 & 10.4909879450 \\
\hline & $a l$ & 0.0000066893 & 0.0040711895 & 0.2611855443 & 7.1200149308 & 0.0158511132 & 0.0204092881 & 0.0134133634 & 2.2441307690 \\
\hline & $a 0$ & 0.0000000258 & 0.0007690826 & 0.0068166056 & 0.9016776896 & 0.0002714832 & 0.0004146653 & 0.0002086911 & 0.1067113398 \\
\hline
\end{tabular}


Table 26 - Plant 1 - Sub-model 3 - Transfer Function Numerator Parameters.

\begin{tabular}{|c|c|c|c|c|c|c|c|c|c|}
\hline$P I$ & $N$ & $u_{I}$ & $u_{2}$ & $u_{3}$ & $u_{4}$ & $u_{5}$ & $u_{6}$ & $u_{7}$ & $d_{l}$ \\
\hline \multirow{6}{*}{$y_{I}$} & $b 5$ & 0 & -0.0000304206 & -0.0004811173 & 0 & 0 & 0 & 0 & 0 \\
\hline & $b 4$ & -0.0967598548 & -0.0009884211 & -0.0296067046 & 0 & 0.0024055083 & 0 & 0 & 0.0038449213 \\
\hline & $b 3$ & 0.0757443938 & 0.0018699441 & -0.0025507392 & 0 & 0.0012435071 & 0 & 0 & -0.0018803664 \\
\hline & $b 2$ & 0.0402694524 & 0.0037816404 & 0.0005873454 & 0.1872027740 & -0.0004363791 & 0.0064371603 & 0.0014603898 & -0.0007214263 \\
\hline & $b 1$ & 0.0008788880 & 0.0011459035 & -0.0000212824 & 0.3804417343 & -0.0020497651 & -0.0003911489 & -0.0016085650 & -0.0000010313 \\
\hline & $b 0$ & 0.0000024289 & -0.0000059921 & 0.0000070799 & 0.0611929572 & -0.0000691372 & -0.0041725982 & -0.0000012559 & -0.0000003004 \\
\hline \multirow{6}{*}{$y_{2}$} & $b 5$ & 0 & 0 & 0 & 0 & 0 & 0 & 0 & 0 \\
\hline & $b 4$ & 0 & 0 & -0.0004595011 & 0 & 0.0009349578 & 0.0964977502 & 0.0004338471 & 0.0010607255 \\
\hline & $b 3$ & 0.1701853788 & 0 & -0.0154080337 & 0.2929186916 & 0.1098390664 & -0.0330707891 & 0.0436993928 & 0.0543642719 \\
\hline & $b 2$ & 0.0645609446 & 0.0785754810 & 0.0209652632 & 0.3446787536 & -0.0402159523 & -0.0325872920 & -0.0149114427 & -0.0195216732 \\
\hline & $b 1$ & 0.0000126727 & 0.1500729702 & 0.0288375261 & 0.5291807252 & -0.0424606605 & -0.0026768588 & -0.0218512442 & -0.0247581780 \\
\hline & $b 0$ & 0.0000240915 & 0.0095622703 & 0.0021209980 & 0.0649327586 & -0.0034825132 & -0.0000007496 & -0.0021296606 & -0.0021241143 \\
\hline \multirow{6}{*}{$y_{3}$} & $b 5$ & 0 & 0 & -0.0034423830 & 0 & 0 & 0 & 0 & 0.0028266091 \\
\hline & b4 & 0.2933262611 & 0 & -0.2178252255 & 0.0012458682 & 0.0032479772 & 0.3126539383 & 0 & 0.2206005931 \\
\hline & $b 3$ & 0.0037663145 & -0.0073530113 & 0.1401979813 & 0.4167266571 & 0.3968248155 & -0.0979865917 & 3.2373336680 & -0.0953843127 \\
\hline & $b 2$ & 0.0006276029 & 0.1388094874 & 0.1968575734 & 0.0859389794 & -0.1491127943 & -0.1453533498 & -1.6801852762 & -0.1096309346 \\
\hline & $b 1$ & 0.0000084007 & 0.2324094432 & 0.0170033709 & 0.0002299544 & -0.1860377963 & -0.0105997862 & -1.4104925956 & -0.0076602082 \\
\hline & $b 0$ & 0.0000000291 & -0.0356017729 & 0.0000143683 & 0.0000466836 & -0.0132415865 & 0.0000001275 & -0.0882192990 & -0.0000777037 \\
\hline \multirow{6}{*}{$y_{4}$} & $b 5$ & 0.0056303574 & 0 & 0 & 0 & 0 & 0.0017757943 & 0.0010649518 & 0 \\
\hline & $b 4$ & -0.5768002099 & 0 & 0 & 0 & 0.0018503252 & 0.1356180743 & 0.0786049685 & 0 \\
\hline & $b 3$ & 0.9627108377 & 0 & -0.0047701229 & 0 & 0.1804136720 & -0.0081378350 & -0.0136045652 & 0.8790667934 \\
\hline & $b 2$ & 0.1807511817 & 0.0851166922 & -0.3508763309 & 0 & -0.0339960560 & -0.1089559456 & -0.0710357052 & -0.1041722242 \\
\hline & $b 1$ & 0.0067432843 & 0.1696093864 & 0.0644862404 & 35.0254446464 & -0.1419415046 & -0.0094952184 & -0.0059001702 & -0.8932084302 \\
\hline & bo & 0.0000094498 & -0.0347332831 & 0.1771092640 & 7.4652959797 & -0.0061583284 & -0.0002858034 & -0.0001489775 & -0.0255336445 \\
\hline \multirow{6}{*}{$y_{5}$} & $b 5$ & 0 & 0 & 0 & 0 & 0 & 0 & 0 & 0 \\
\hline & $b 4$ & -0.0997037821 & 0 & -0.4500685627 & 0 & 0.0902973172 & 0 & 0.0412096157 & 0 \\
\hline & $b 3$ & 0.1459416038 & 0 & 0.4059725295 & 0 & 0.0191763759 & 0.1771056001 & 0.0123993562 & 0 \\
\hline & $b 2$ & 0.0334931150 & 0.0530276560 & -0.0046429290 & 35.2806476778 & -0.1161101580 & -0.1920668770 & -0.0584595608 & 0.2172711511 \\
\hline & bl & 0.0000392396 & 0.1334069169 & 0.0047835183 & 16.4295202161 & 0.0000021175 & -0.0111699596 & -0.0062537222 & -0.2777015389 \\
\hline & $b 0$ & 0.0000025036 & -0.0324804720 & 0.0000139824 & 0.1260652020 & -0.0000014995 & -0.0007044555 & -0.0003545904 & -0.0220038195 \\
\hline \multirow{6}{*}{$y_{6}$} & $b 5$ & 0 & 0 & 0 & 0 & 0 & 0 & 0 & 0 \\
\hline & $b 4$ & -0.0004675831 & 0 & 0 & 0 & 0 & 0.0007157070 & 0.0004893828 & 0.0823001165 \\
\hline & $b 3$ & -0.0666103798 & 0 & -0.2676786259 & 0 & 0.1618401644 & 0.1228331290 & 0.0708239799 & -0.0313958109 \\
\hline & $b 2$ & 0.2087545187 & 0.0472180377 & 0.1795643659 & 0 & -0.0598624839 & -0.0378395231 & -0.0244844278 & -0.0400162512 \\
\hline & $b 1$ & 0.2074568881 & 0.0881291009 & 0.2066113331 & 17.2795835259 & -0.0652118665 & -0.0511321408 & -0.0335057212 & -0.0046856523 \\
\hline & $b 0$ & 0.0131703726 & -0.0118095499 & 0.0001926803 & 2.0582675939 & -0.0040324235 & -0.0033729533 & -0.0022635411 & -0.0001764549 \\
\hline \multirow{6}{*}{$y_{7}$} & $b 5$ & 0 & 0 & 0 & 0.0003960432 & 0 & 0 & 0 & 0 \\
\hline & $b 4$ & 0 & 0 & 0 & 0.0636796773 & 0 & 0 & 0 & 0 \\
\hline & $b 3$ & 0.0741208265 & 0 & 0 & 0.0173826029 & 0 & 0.5394954237 & 0.3635085128 & 0.4112716783 \\
\hline & $b 2$ & 0.0328476819 & 0.0172579835 & -2.6721977459 & 0.0002649429 & 0.7420823303 & 0.0107212801 & 0.0530503394 & 0.1673423923 \\
\hline & $b 1$ & 0.0000045688 & 0.0216876943 & -2.5838894295 & 0.0000227459 & 0.0001344630 & -0.0739762568 & -0.0364941213 & -0.0623591554 \\
\hline & $b 0$ & 0.0000089132 & -0.0052030159 & -0.2055267629 & 0.0000000139 & -0.0794124225 & -0.0029112182 & -0.0013249714 & -0.0023645576 \\
\hline \multirow{6}{*}{$y_{8}$} & $b 5$ & 0 & 0 & 0 & 0 & 0 & -0.0063925466 & 0 & 0 \\
\hline & $b 4$ & 0 & 0 & 0 & 0.0001026033 & 0 & 0.4701152233 & 0 & 0 \\
\hline & $b 3$ & 0 & 0.0049959836 & 0.0018195983 & 0.0000332704 & 0 & -0.5212368032 & 0.0241700324 & -0.0230110074 \\
\hline & $b 2$ & 0.2411165069 & 0.0117398640 & 0.0000092617 & 0.0000004157 & 0.0866805382 & 0.0001641637 & -0.0408932188 & -0.0135836427 \\
\hline & $b 1$ & 0.0287416034 & -0.0056688548 & 0.0000061724 & 0.0000001260 & -0.1258846718 & -0.0012919484 & -0.0000201526 & -0.0003656413 \\
\hline & $b 0$ & 0.0000004719 & -0.0000551889 & -0.0000000061 & 03 & -0.0000749529 & -0.0000014223 & -0.0000010659 & -0.0000170570 \\
\hline \multirow{6}{*}{$y_{9}$} & $b 5$ & 0 & 0 & 0 & 0 & 0 & 0 & 0 & 0 \\
\hline & $b 4$ & 0.0497344087 & 0 & -0.0033056454 & -0.0000125879 & 0 & 0 & 0 & 0 \\
\hline & $b 3$ & 0.0058706050 & 0.0020056980 & 0.0023038402 & -0.0006560784 & -0.0198088948 & 0 & 0 & 0 \\
\hline & $b 2$ & 0.0001555260 & 0.0040816319 & 0.0023817555 & 0.0001722519 & -0.0041790486 & -0.0278235870 & -0.0164384305 & -0.0686159728 \\
\hline & $b 1$ & 0.0000124831 & -0.0002274497 & 0.0007858726 & 0.0000132644 & -0.0000527292 & -0.0022620946 & -0.0014284604 & -0.1063029955 \\
\hline & $b 0$ & 0.0000000658 & -0.0000713210 & 0.0000351091 & 0.0000174236 & -0.0000102258 & -0.0000017045 & -0.0000002121 & -0.0086614055 \\
\hline \multirow{6}{*}{$y_{10}$} & $b 5$ & 0 & 0 & 0 & 0 & 0 & 0.0002468965 & 0 & 0 \\
\hline & $b 4$ & 0.0497222865 & 0 & -0.0018456209 & 0 & 0.0771085143 & 0.0530115805 & 0 & 0 \\
\hline & $b 3$ & 0.1624272265 & 0 & 0.0018350115 & -0.0061221821 & 0.0007292580 & -0.0056877641 & 12.9621447071 & 0.0360322429 \\
\hline & $b_{2}$ & 0.0011747171 & 0.0420119747 & 0.0006960273 & 0.0017809492 & -0.0327787949 & -0.0252289037 & 20.1871287867 & -0.0204498601 \\
\hline & $b 1$ & 0.0001387676 & -0.0186320257 & 0.0004168678 & 0.0000613299 & -0.0013168704 & -0.0012354086 & -14.3512831331 & -0.0039670023 \\
\hline & $b 0$ & 0.0000007692 & -0.0006685108 & -0.0000000615 & 0.0000001408 & -0.0000100117 & -0.0000008527 & -1.1353433423 & -0.0001378550 \\
\hline \multirow{6}{*}{$y_{H I}$} & $b 5$ & 0 & 0 & -0.0000934202 & -0.0000183621 & 0 & 0.0005417753 & 0.0003286385 & 0 \\
\hline & $b 4$ & 0.1365172836 & -0.0000661001 & -0.0046557073 & -0.0018390046 & 0 & 0.0447057305 & 0.0237660745 & 0 \\
\hline & $b 3$ & 0.0262371205 & -0.0026124443 & 0.0034097977 & -0.0000195326 & 0.0680361355 & -0.0388019267 & -0.0210196327 & 0 \\
\hline & $b 2$ & 0.0014844508 & 0.0090516703 & 0.0023949468 & -0.0000981210 & -0.0643536292 & -0.0657235635 & -0.0372750394 & -0.0262663223 \\
\hline & $b 1$ & 0.0000175874 & 0.0063418806 & 0.0010802997 & 0.0001812689 & -0.0837513303 & -0.0024093602 & -0.0016661070 & -0.0405761206 \\
\hline & bo & 0.0000009636 & -0.0066301879 & 0.0003404349 & 0.0000072024 & -0.0044569281 & -0.0000115763 & -0.0000292552 & -0.0021460877 \\
\hline
\end{tabular}


Table 27 - Plant 1 - Sub-model 3 - Transfer Function Denominator Parameters.

\begin{tabular}{|c|c|c|c|c|c|c|c|c|c|}
\hline$P I$ & $D$ & $u_{I}$ & $u_{2}$ & $u_{3}$ & $u_{4}$ & $u_{5}$ & $u_{6}$ & $u_{7}$ & $d_{l}$ \\
\hline \multirow{6}{*}{$y_{I}$} & $a 5$ & 1 & 1 & 1 & 0 & 1 & 0 & 0 & 1 \\
\hline & a4 & 22.3899483137 & 1.8265912935 & 2.2621052653 & 1 & 1.3686827472 & 1 & 0 & 2.2188077140 \\
\hline & $a 3$ & 8.3232794084 & 2.4632781813 & 0.6522204290 & 6.2568572818 & 1.7391254410 & 2.6458118104 & 1 & 0.6119921057 \\
\hline & $a_{2}$ & 0.7685965336 & 1.3732289193 & 0.0682333687 & 19.5679070116 & 0.8222238684 & 4.5861236843 & 1.0794972534 & 0.0459516726 \\
\hline & $a I$ & 0.0136561983 & 0.3389528802 & 0.0052685580 & 18.4285107472 & 0.0935949087 & 2.2162871662 & 0.1085460142 & 0.0002548693 \\
\hline & $a 0$ & 0.0000334310 & 0.0249025025 & 0.0004067646 & 1.9822943182 & 0.0022716357 & 0.1908772561 & 0.0000733851 & 0.0000187628 \\
\hline \multirow{6}{*}{$y_{2}$} & $a 5$ & 1 & 1 & 0 & 0 & 0 & 1 & 0 & 0 \\
\hline & a4 & 1.7900687557 & 3.1856999094 & 1 & 1 & 1 & 2.0984813991 & 1 & 1 \\
\hline & $a 3$ & 1.2270688810 & 7.3488314417 & 1.4352434028 & 11.4776718308 & 1.8682055980 & 1.8098983768 & 1.7244453505 & 1.9264305955 \\
\hline & $a 2$ & 0.1265876627 & 7.6708633142 & 1.8543864029 & 14.9137499280 & 1.6601539493 & 0.3577383636 & 1.6549080429 & 1.8028381211 \\
\hline & $a l$ & 0.0004472899 & 5.2179139993 & 0.5316387653 & 15.7616425268 & 0.3408810659 & 0.0182207835 & 0.3888063675 & 0.3816696142 \\
\hline & $a 0$ & 0.0000459052 & 0.6880023792 & 0.0298542604 & 1.7179647670 & 0.0172865146 & 0.0000048123 & 0.0221496386 & 0.0196212800 \\
\hline \multirow{6}{*}{$y_{3}$} & $a 5$ & 1 & 1 & $I$ & 0 & 0 & 1 & 1 & $I$ \\
\hline & $a 4$ & 0.2179810997 & 2.7787750234 & 2.0469883594 & 1 & 1 & 1.9691972317 & 18.4321962481 & 2.0300083531 \\
\hline & $a 3$ & 0.0048087394 & 6.1909271899 & 2.0348118471 & 2.0498828666 & 1.8502269999 & 1.7568554441 & 32.8891545138 & 1.8278414768 \\
\hline & $a 2$ & 0.0004679240 & 6.1432122167 & 0.5249571299 & 0.3357303695 & 1.6411551689 & 0.4154127254 & 26.8727831046 & 0.4162581158 \\
\hline & $a l$ & 0.0000056830 & 4.1716362571 & 0.0320222242 & 0.0011146151 & 0.3811533771 & 0.0210711783 & 5.7092531710 & 0.0217820114 \\
\hline & $a 0$ & 0.0000000252 & 0.7524431903 & 0.0000277954 & 0.0001822563 & 0.0191512119 & 0.0000006419 & 0.2631691110 & 0.0002094602 \\
\hline \multirow{6}{*}{$y_{4}$} & $a 5$ & 1 & 1 & 0 & 0 & 0 & 1 & 1 & 1 \\
\hline & $a 4$ & 4.3926740048 & 2.9554371944 & 0 & 1 & 1 & 1.7096616009 & 1.5753533015 & 8.9656148171 \\
\hline & $a 3$ & 1.3497299191 & 5.6157649824 & 1 & 16.5747694418 & 1.6077899917 & 1.4845143836 & 1.3873363288 & 16.4311633450 \\
\hline & $a 2$ & 0.1247732517 & 5.6083217038 & 2.3056953589 & 96.9952971583 & 1.3370636703 & 0.2816318963 & 0.2619603330 & 15.3285149203 \\
\hline & al & 0.0034979787 & 3.0264533364 & 1.6084581270 & 138.0003649608 & 0.2059178707 & 0.0174308556 & 0.0154048820 & 2.1937296088 \\
\hline & $a 0$ & 0.0000050387 & 0.3477638403 & 0.1934240529 & 16.6866512432 & 0.0064517797 & 0.0004142638 & 0.0003196828 & 0.0497641779 \\
\hline \multirow{6}{*}{$y_{5}$} & $a 5$ & 0 & 1 & $I$ & 0 & 1 & 1 & 1 & 0 \\
\hline & a4 & 1 & 3.1978256166 & 3.3264923611 & 1 & 1.4909592006 & 2.1943011128 & 1.6394881605 & 1 \\
\hline & $a 3$ & 0.2662668467 & 5.5948892543 & 0.4058937291 & 125.7762211227 & 1.2544136085 & 2.9439131469 & 1.4576661989 & 4.5127786490 \\
\hline & $a 2$ & 0.0178015161 & 5.6700829611 & 0.0400721787 & 290.6294570245 & 0.1164251580 & 0.4531322357 & 0.2665183039 & 5.8082702559 \\
\hline & al & 0.0000293075 & 2.6256615633 & 0.0046148862 & 33.4964716501 & 0.0000178378 & 0.0262942903 & 0.0206585024 & 0.9739688662 \\
\hline & $a 0$ & 0.0000015131 & 0.2398736313 & 0.0000131932 & 0.2437732444 & 0.0000016270 & 0.0009745806 & 0.0007241222 & 0.0409429484 \\
\hline \multirow{6}{*}{$y_{6}$} & $a 5$ & 0 & 1 & 1 & 1 & 0 & 0 & 0 & 1 \\
\hline & a4 & 1 & 2.9687600653 & 5.1363763013 & 8.7468269673 & 1 & 1 & 1 & 2.2012783410 \\
\hline & $a 3$ & 2.1986666535 & 6.5121772051 & 9.5874594894 & 50.1355839555 & 2.0532219181 & 2.1244872525 & 1.9717565629 & 2.1442537286 \\
\hline & $a 2$ & 2.4118909634 & 6.9704355677 & 9.8230124027 & 175.6137535982 & 1.8805096470 & 1.9819517242 & 1.8837612807 & 0.6212532068 \\
\hline & al & 0.6603196982 & 4.8191217801 & 2.0158038919 & 243.1102517175 & 0.4646944714 & 0.5083553999 & 0.5015072532 & 0.0555289334 \\
\hline & $a 0$ & 0.0315294019 & 1.0743592950 & 0.0018851559 & 25.0518332694 & 0.0219476851 & 0.0251166753 & 0.0255314784 & 0.0017656474 \\
\hline \multirow{6}{*}{$y_{7}$} & $\overline{a 5}$ & $I$ & $I$ & 0 & 1 & 0 & 1 & 1 & 1 \\
\hline & a4 & 1.2899678802 & 1.9184020491 & $I$ & 2.1674124994 & 1 & 5.4389150316 & 4.6753639680 & 4.9282169350 \\
\hline & $a 3$ & 0.8673357614 & 4.2417780091 & 4.4327748772 & 0.3095053000 & 4.8962259116 & 10.6412167244 & 9.2202257761 & 10.5263886655 \\
\hline & $a_{2}$ & 0.0985143015 & 3.4368956134 & 10.8115252479 & 0.0055560015 & 9.4990757359 & 7.2055825498 & 6.3065116156 & 9.1990270845 \\
\hline & al & 0.0002230907 & 2.1871098925 & 8.4882439095 & 0.0003864341 & 5.9524896069 & 1.0459071934 & 0.9013319349 & 1.4996624341 \\
\hline & $a 0$ & 0.0000252535 & 0.2744672723 & 1.0371296677 & 0 & 0.6349777324 & 0.0301310210 & 0.0236729795 & 0.0397025529 \\
\hline \multirow{6}{*}{$y_{8}$} & a5 & 0 & $I$ & 0 & 1 & 0 & 1 & 0 & $I$ \\
\hline & a4 & 1 & 1.9127912654 & 1 & 0.2404627238 & 0 & 12.7819786998 & 1 & 1.1868208477 \\
\hline & $a 3$ & 1.7274488271 & 2.2769193141 & 0.1170897031 & 0.0234804562 & 1 & 1.4452951753 & 1.6959629572 & 0.5703454519 \\
\hline & $a 2$ & 0.3601226890 & 1.2995303429 & 0.0044119139 & 0.0008934976 & 2.3675030916 & 0.0330306386 & 0.1908760472 & 0.0617919084 \\
\hline & $a l$ & 0.0198803570 & 0.1562441146 & 0.0003781282 & 0.0000698780 & 0.2714326500 & 0.0036430045 & 0.0000595262 & 0.0019087334 \\
\hline & $a 0$ & 0 & 0.0013521820 & 0.0000000640 & 0.0000001020 & 0 & 0.0000015311 & 0.0000066599 & 0.0000608017 \\
\hline \multirow{6}{*}{$y_{9}$} & $a 5$ & 1 & 1 & $I$ & 0 & 1 & 1 & 1 & 1 \\
\hline & $a 4$ & 0.2121624978 & 1.6506552801 & 2.6698689789 & 1 & 1.3344287230 & 1.3806923892 & 1.3202965809 & 6.3565688815 \\
\hline & $a 3$ & 0.0133927061 & 2.4192547112 & 3.0940355058 & 1.1915448950 & 0.4445537426 & 2.5422022003 & 2.4650823992 & 12.8692140780 \\
\hline & $a 2$ & 0.0004634203 & 1.5922231387 & 2.1636563884 & 1.8246936667 & 0.0292980631 & 0.4635913032 & 0.4746295994 & 13.5279587427 \\
\hline & al & 0.0000235113 & 0.3900713193 & 0.2998871567 & 0.3548812223 & 0.0011240103 & 0.0179252534 & 0.0189117869 & 2.2920221761 \\
\hline & $a 0$ & 0.0000000469 & 0.0222601207 & 0.0093653809 & 0.0686911608 & 0.0000619218 & 0.0000155961 & 0.0000046286 & 0.0876309853 \\
\hline \multirow{6}{*}{$y_{10}$} & a5 & 1 & I & 1 & 0 & 1 & 1 & 1 & 0 \\
\hline & a4 & 3.1879133674 & 12.4770097957 & 1.7089162274 & 1 & 3.2420730370 & 3.2965395506 & 552.7986058614 & 1 \\
\hline & $a 3$ & 0.3799787766 & 13.1655631524 & 2.1072350289 & 14.9867882816 & 2.5697749554 & 2.5590566726 & 1447.9103568379 & 2.9666748932 \\
\hline & $a 2$ & 0.0046729179 & 14.7682934904 & 1.2821375169 & 5.7470030424 & 0.3601600697 & 0.3750967472 & 2405.7514833214 & 0.8408439430 \\
\hline & $a I$ & 0.0003195953 & 2.9155807504 & 0.1231764223 & 0.1517361862 & 0.0104679260 & 0.0118230676 & 459.7186921792 & 0.0716036185 \\
\hline & $a 0$ & 0.0000012435 & 0.0792449635 & 0 & 0.0003111686 & 0.0000788441 & 0.0000104738 & 19.0051696861 & 0.0017808717 \\
\hline \multirow{6}{*}{$y_{I I}$} & $a 5$ & 1 & 0 & 1 & 1 & 0 & 1 & 1 & 0 \\
\hline & $a 4$ & 0.3404266928 & 1 & 1.7923237081 & 1.5912334123 & 1 & 2.3830874281 & 2.1114267401 & 1 \\
\hline & $a 3$ & 0.0379420946 & 2.1003810462 & 2.7165020411 & 2.2958893268 & 2.5670401905 & 2.3117101431 & 2.1562798640 & 1.8859937841 \\
\hline & $a 2$ & 0.0015905250 & 2.7656634273 & 1.7377825637 & 1.2756813233 & 2.2835377288 & 0.3748704352 & 0.3678869607 & 1.8695312487 \\
\hline & $a l$ & 0.0000257336 & 1.8645354845 & 0.6011589015 & 0.2374295248 & 0.3953766413 & 0.0106934623 & 0.0135512492 & 0.3297399120 \\
\hline & $a 0$ & 0.0000009356 & 0.3748473374 & 0.0559731116 & 0.0077789922 & 0.0145339932 & 0.0000527354 & 0.0002116879 & 0.0120333511 \\
\hline
\end{tabular}


Table 28 - Plant 2 - Sub-model 1 - Transfer Function Numerator Parameters.

\begin{tabular}{|c|c|c|c|c|c|c|c|c|c|}
\hline$P I$ & $N$ & $u_{1}$ & $u_{2}$ & $u_{3}$ & $u_{4}$ & $u_{5}$ & $u_{6}$ & $u_{7}$ & $d_{l}$ \\
\hline \multirow{6}{*}{$y_{I}$} & $b 5$ & 0 & 0 & 0 & 0 & 0 & 0 & 0 & 0 \\
\hline & $b 4$ & 0 & 0 & 0 & 0 & 0 & 0 & 0 & 0 \\
\hline & $b 3$ & 0 & 0.0000105592 & 0 & 0.0006485585 & -0.0000530434 & 0 & 0 & 0 \\
\hline & $b_{2}$ & 0.0002021277 & 0.0008053314 & 0.0001090997 & 0.0001103545 & -0.0001276728 & 0 & 0 & 0 \\
\hline & $b 1$ & -0.0000008793 & 0.0000616034 & 0.0000001065 & 0.0000014156 & -0.0000019503 & 0 & 0 & 0 \\
\hline & $b 0$ & 0.0000000276 & -0.0000022755 & 0.0000000037 & 0.0000002450 & -0.0000000157 & 0 & 0 & 0 \\
\hline \multirow{6}{*}{$y_{2}$} & $b 5$ & 0 & 0 & 0.0000333343 & 0 & 0 & 0 & 0 & 0 \\
\hline & $b 4$ & 0 & -0.0011797225 & -0.0028658019 & 0 & -0.0001033887 & 0 & 0 & 0 \\
\hline & $b 3$ & 0 & 0.0020624122 & -0.0013776906 & 0 & 0.0002552216 & 0 & 0 & 0 \\
\hline & $b_{2}$ & 0.0003760169 & 0.0005599782 & 0.0047383111 & 0.0025884668 & -0.0002887653 & 0 & 0 & 0 \\
\hline & $b 1$ & -0.0000012762 & 0.0000075953 & -0.0000020041 & -0.0000006613 & -0.0000064880 & 0 & 0 & 0 \\
\hline & $b 0$ & 0.0000000748 & 0.0000010915 & 0.0000016630 & 0.0000044051 & -0.0000000470 & 0 & 0 & 0 \\
\hline \multirow{6}{*}{$y_{3}$} & $b 5$ & 0 & 0 & -0.0002605893 & 0 & -0.0002379648 & 0 & 0 & 0 \\
\hline & $b 4$ & 0 & 0 & -0.0033864604 & 0 & 0.0012166461 & 0 & 0 & 0 \\
\hline & $b 3$ & 0 & 0 & 0.0026076766 & 0.0031594354 & -0.0005156922 & 0 & 0 & 0 \\
\hline & $b 2$ & 0.0023543340 & 0.0049545352 & 0.0012502043 & 0.0019575861 & -0.0004600914 & 0 & 0 & 0 \\
\hline & $b 1$ & -0.0000124809 & -0.0001814582 & 0.0000049563 & 0.0000162856 & -0.0000467351 & 0 & 0 & 0 \\
\hline & bo & 0.0000004465 & -0.0000315574 & 0.0000012144 & 0.0000000240 & -0.0000001554 & 0 & 0 & 0 \\
\hline \multirow{6}{*}{$y_{4}$} & $b 5$ & 0 & 0 & 0 & 0 & 0 & 0 & 0 & 0 \\
\hline & $b 4$ & 0.0005437500 & 0 & -0.0008883635 & 0 & 0.0007678622 & 0 & 0 & 0 \\
\hline & $b 3$ & 0.0007419398 & 0 & -0.0027730092 & 0.0176006554 & -0.0006663475 & 0 & 0 & 0 \\
\hline & $b_{2}$ & 0.0021915415 & 0.0078522676 & 0.0023060602 & 0.0055162226 & -0.0000031747 & 0 & 0 & 0 \\
\hline & $b 1$ & -0.0000125387 & -0.0006204302 & -0.0000010161 & 0.0006891328 & -0.0000014867 & 0 & 0 & 0 \\
\hline & $b 0$ & 0.0000004024 & -0.0000577645 & 0.0000015692 & 0.0000007035 & -0.0000000037 & 0 & 0 & 0 \\
\hline \multirow{6}{*}{$y_{5}$} & $b 5$ & 0 & 0.0002257902 & 0.0000747327 & 0 & -0.0001335956 & 0 & 0 & 0 \\
\hline & $b 4$ & 0.0010501639 & -0.0006795354 & -0.0012156465 & 0 & 0.0008051448 & 0 & 0 & 0 \\
\hline & $b 3$ & -0.0002100744 & 0.0022503538 & 0.0008367428 & -0.0001970067 & -0.0004396091 & 0 & 0 & 0 \\
\hline & $b 2$ & 0.0007886041 & -0.0002568101 & 0.0000331362 & 0.0026969878 & -0.0000086461 & 0 & 0 & 0 \\
\hline & $b 1$ & -0.0000041693 & 0.0000001108 & 0.0000006100 & 0.0009504691 & -0.0000020170 & 0 & 0 & 0 \\
\hline & bo & 0.0000001352 & -0.0000007408 & 0.0000000226 & 0.0000005902 & 0.0000000024 & 0 & 0 & 0 \\
\hline \multirow{6}{*}{$y_{6}$} & $b 5$ & 0 & 0 & 0 & 0 & 0 & 0 & 0 & 0 \\
\hline & $b 4$ & -0.0010427388 & 0 & -0.0004883568 & 0.0001517918 & 0 & 0 & 0 & 0 \\
\hline & $b 3$ & 0.0015805409 & 0 & 0.0006906983 & 0.0011194126 & 0 & 0.0018713711 & 0 & 0 \\
\hline & $b_{2}$ & 0.0013186353 & 0 & 0.0000903036 & 0.0039374246 & 0.0016684874 & -0.0017119642 & 0.0017174627 & -0.0034511624 \\
\hline & $b 1$ & -0.0000034714 & 0.0048981531 & 0.0000018591 & 0.0002826369 & -0.0020450245 & -0.0000662931 & -0.0020575607 & -0.0000434738 \\
\hline & $b 0$ & 0.0000001334 & -0.0004532158 & 0.0000000811 & 0.0000141302 & -0.0000000070 & -0.0000000993 & -0.0000041105 & -0.0000090459 \\
\hline \multirow{6}{*}{$y_{7}$} & $b 5$ & 0 & 0 & 0 & 0 & 0 & 0 & 0 & 0 \\
\hline & $b 4$ & 0 & 0 & 0 & 0 & 0 & 0 & 0 & 0 \\
\hline & $b 3$ & 0.0665698364 & 0 & 0 & 0.1471002289 & 0.2888052733 & 0.5773483454 & 0 & 0.3820623195 \\
\hline & $b 2$ & 0.0281275457 & 0.1501707398 & -2.6051938439 & 0.0248314830 & 0.0121883336 & -0.0438761340 & 0.2430387054 & -0.0151120690 \\
\hline & bl & 0.0000274984 & 0.0914268778 & -2.6286378281 & 0.0000097100 & -0.0244003082 & -0.0498693842 & 0.0073465225 & 0.0005123089 \\
\hline & $b 0$ & 0.0000082372 & -0.0192359460 & -0.2108396101 & 0.0000004808 & -0.0006675078 & -0.0001483825 & -0.0137453794 & -0.0000773529 \\
\hline \multirow{6}{*}{$y_{8}$} & $b 5$ & -0.0090656644 & 0 & 0.0000932625 & -0.0020177510 & -0.0058362569 & 0 & 0 & 0 \\
\hline & $b 4$ & 0.0721577293 & 0 & -0.0028639391 & 0.0000944744 & 0.0398799246 & 0 & 0 & 0 \\
\hline & $b 3$ & 0.0026057135 & 0 & 0.0031608319 & 0.0001013188 & -0.0302840739 & -0.0196564171 & -0.0235121585 & -0.0103305061 \\
\hline & $b 2$ & 0.0001465065 & 0 & 0.0021929233 & 0.0000028536 & -0.0554138279 & -0.0038477133 & -0.0027188833 & -0.0001860717 \\
\hline & $b 1$ & 0.0000047252 & 0.5407470620 & 0.0013435382 & 0.0000004508 & -0.0009972414 & -0.0000364645 & -0.0000629111 & -0.0000317251 \\
\hline & bo & 0.0000000039 & -0.1212838147 & 0.0000392868 & 0.0000000042 & -0.0000066728 & -0.0000070346 & -0.0000071390 & -0.0000005620 \\
\hline \multirow{6}{*}{$y_{9}$} & $b 5$ & 0 & 0.0000003985 & 0 & 0 & 0 & 0 & 0 & 0 \\
\hline & $b 4$ & 0 & -0.0000606421 & 0.0000084610 & 0 & 0 & 0.0001015675 & 0.0000309521 & -0.0003126651 \\
\hline & $b 3$ & 0 & 0.0002750513 & 0.0000030143 & 0 & 0 & 0.0000588567 & -0.0001166646 & -0.0001356060 \\
\hline & $b_{2}$ & 0 & -0.0000127488 & 0.0000000685 & 0 & -0.0012679443 & -0.0001214780 & -0.0000524500 & -0.0000512510 \\
\hline & $b 1$ & 0.0083630022 & -0.0000006064 & 0.0000000113 & 0.0000005850 & 0.0000245838 & 0.0000001386 & -0.0000000675 & -0.0000003677 \\
\hline & $b 0$ & 0.0026028561 & -0.0000001119 & 01 & 0.0000001041 & -0.0001907248 & -0.0000000289 & -0.0000000179 & -0.0000000963 \\
\hline \multirow{6}{*}{$y_{10}$} & $b 5$ & 0 & 0 & 0 & -0.0000784936 & 0 & 0 & 0.0001944190 & 0 \\
\hline & $b 4$ & 0.0280751511 & 0.0000483327 & 0 & 0.0003485420 & 0.0002551417 & 0 & 0.3027194636 & 0 \\
\hline & $b 3$ & 0.0058796909 & -0.0157766891 & 0.0042810907 & -0.0000068064 & 0.0479136684 & 0.2029774843 & -0.0954799828 & 0.0719437826 \\
\hline & $b_{2}$ & 0.0000969388 & 0.0387891436 & 0.0000236632 & 0.0000023508 & -0.0036102989 & -0.1178633076 & -0.0239908233 & -0.0320769069 \\
\hline & $b 1$ & 0.0000063278 & -0.0033572042 & 0.0001435389 & -0.0000000115 & -0.0195869412 & -0.0006042024 & -0.0001064624 & -0.0005960677 \\
\hline & $b 0$ & 0.0000000348 & -0.0013626740 & 0.0000003119 & 0.0000000015 & -0.0006445468 & -0.0001476188 & -0.0000068921 & -0.0000855542 \\
\hline \multirow{6}{*}{$y_{I I}$} & $b 5$ & 0 & -0.0000192161 & 0 & 0.0000053272 & 0.0001771473 & 0 & 0 & 0 \\
\hline & $b 4$ & 0 & 0.0001549295 & 0.0000144473 & 0.0000122718 & 0.0000808719 & 0 & -0.0000487828 & 0 \\
\hline & $b 3$ & 0 & 0.0002944829 & -0.0000078867 & 0.0000004165 & -0.0001121576 & 0 & 0.0002000716 & -0.0000227317 \\
\hline & $b_{2}$ & -0.0069739157 & -0.0000916487 & 0.0000197535 & 0.0000000540 & -0.0000009131 & 0 & -0.0001960566 & -0.0000837834 \\
\hline & $b 1$ & 0.0018871296 & -0.0000017362 & -0.0000003185 & 06 & -0.0000014968 & -0.0002871898 & -0.0000059933 & -0.0000011680 \\
\hline & bo & -0.0000175362 & -0.0000002145 & 0.0000071819 & 0 & -0.0000000223 & -0.0000012773 & -0.0000000016 & -0.0000002729 \\
\hline
\end{tabular}


Table 29 - Plant 2 - Sub-model 1 - Transfer Function Denominator Parameters.

\begin{tabular}{|c|c|c|c|c|c|c|c|c|c|}
\hline$P I$ & $D$ & $u_{l}$ & $u_{2}$ & $u_{3}$ & $u_{4}$ & $u_{5}$ & $u_{6}$ & $u_{7}$ & $d_{I}$ \\
\hline \multirow{6}{*}{$y_{t}$} & $a 5$ & 0 & 1 & 0 & 1 & 1 & 0 & 0 & 0 \\
\hline & a4 & 1 & 1.3507773846 & 1 & 2.0505333586 & 2.5459409282 & 0 & 0 & 0 \\
\hline & $a 3$ & 0.1389741430 & 1.1178069763 & 0.1278988268 & 0.2432815790 & 0.3156891595 & 0 & 0 & 0 \\
\hline & $a 2$ & 0.0012515215 & 0.2175232032 & 0.0021193276 & 0.0081242947 & 0.0086520647 & 0 & 0 & 0 \\
\hline & al & 0.0000122512 & 0.0134499920 & 0.0000045124 & 0.0005295602 & 0.0000971112 & 0 & 0 & 0 \\
\hline & $a 0$ & 0.0000001026 & 0.0001699655 & 0.0000000735 & 0.0000079519 & 0.0000005114 & 1 & 1 & 1 \\
\hline \multirow{6}{*}{$y_{2}$} & $a 5$ & 0 & 1 & 1 & 0 & 0 & 0 & 0 & 0 \\
\hline & a4 & 1 & 1.0321925954 & 16.8526437466 & 1 & 1 & 0 & 0 & 0 \\
\hline & $a 3$ & 0.2235842644 & 0.2887153565 & 7.0514227451 & 4.8607540969 & 0.2562881211 & 0 & 0 & 0 \\
\hline & $a 2$ & 0.0017588627 & 0.0092619440 & 0.1160946870 & 0.0891436860 & 0.0080387517 & 0 & 0 & 0 \\
\hline & $a l$ & 0.0000445817 & 0.0005933967 & 0.0024769828 & 0.0082455499 & 0.0001178249 & 0 & 0 & 0 \\
\hline & $a 0$ & 0.0000001417 & 0.0000102265 & 0.0000387033 & 0.0001483415 & 0.0000003869 & 1 & 1 & 1 \\
\hline \multirow{6}{*}{$y_{3}$} & $a 5$ & 0 & 1 & 1 & 0 & 1 & 0 & 0 & 0 \\
\hline & $a 4$ & 1 & 1.3256143351 & 0.8770312258 & 1 & 0.8030191512 & 0 & 0 & 0 \\
\hline & $a_{3}$ & 0.3491007758 & 1.2309820553 & 0.1519942911 & 0.4178283212 & 0.2059811911 & 0 & 0 & 0 \\
\hline & $a 2$ & 0.0023434779 & 0.3371775272 & 0.0034117767 & 0.0099103709 & 0.0161713210 & 0 & 0 & 0 \\
\hline & $a l$ & 0.0000482234 & 0.0198294963 & 0.0001494529 & 0.0000648156 & 0.0002609199 & 0 & 0 & 0 \\
\hline & $a 0$ & 0.0000003046 & 0.0002560674 & 0.0000022327 & 0.0000001167 & 0.0000007662 & 1 & 1 & 1 \\
\hline \multirow{6}{*}{$y_{4}$} & $a 5$ & 0 & 1 & 0 & 1 & 1 & 0 & 0 & 0 \\
\hline & $a 4$ & 1 & 1.6024388138 & 1 & 1.7965021318 & 0.1648570086 & 0 & 0 & 0 \\
\hline & $a 3$ & 0.1471268816 & 1.2565771703 & 0.1738836809 & 0.5288161072 & 0.0056337703 & 0 & 0 & 0 \\
\hline & $a 2$ & 0.0009963385 & 0.2304979257 & 0.0032637295 & 0.0541375393 & 0.0003659525 & 0 & 0 & 0 \\
\hline & $a l$ & 0.0000188194 & 0.0108847753 & 0.0001191037 & 0.0008067949 & 0.0000060483 & 0 & 0 & 0 \\
\hline & $a 0$ & 0.0000001111 & 0.0001337089 & 0.0000017663 & 0.0000012991 & 0.0000000097 & 1 & 1 & 1 \\
\hline \multirow{6}{*}{$y_{5}$} & a5 & 0 & 1 & 1 & 0 & 1 & 0 & 0 & 0 \\
\hline & $a 4$ & 1 & 0.7430327125 & 0.1489796612 & 0 & 0.1485903016 & 0 & 0 & 0 \\
\hline & $a 3$ & 0.0998199022 & 0.0852072696 & 0.0071800090 & 1 & 0.0087500236 & 0 & 0 & 0 \\
\hline & $a 2$ & 0.0008420395 & 0.0044157477 & 0.0001622476 & 0.1204811684 & 0.0005913750 & 0 & 0 & 0 \\
\hline & $a l$ & 0.0000103375 & 0.0001862951 & 0.0000044619 & 0.0018161334 & 0.0000058419 & 0 & 0 & 0 \\
\hline & $a 0$ & 0.0000000765 & 0.0000028488 & 0.0000000411 & 0.0000020726 & 0.0000000325 & 1 & 1 & 1 \\
\hline \multirow{6}{*}{$y_{6}$} & $a 5$ & 0 & 0 & 1 & 0 & 1 & 1 & 1 & 1 \\
\hline & $a 4$ & 1 & 1 & 0.6473935561 & 1 & 1.2903523520 & 2.4446258986 & 1.4683268549 & 10.4732031134 \\
\hline & $a 3$ & 0.2653296007 & 1.5431967181 & 0.0773744616 & 1.5432376945 & 2.5683166616 & 1.0772813419 & 2.8901158270 & 3.7222645358 \\
\hline & $a 2$ & 0.0031078612 & 1.3283514917 & 0.0026113749 & 0.1361410524 & 0.9198969436 & 0.0510432746 & 1.0832577634 & 0.1236758551 \\
\hline & $a l$ & 0.0000193241 & 0.3767487921 & 0.0000819485 & 0.0073189983 & 0.0148284997 & 0.0006577167 & 0.0195003172 & 0.0102044684 \\
\hline & $a 0$ & 0.0000001729 & 0.0062836388 & 0.0000010081 & 0.0000951577 & 0.0000035217 & 0.0000004053 & 0.0000402803 & 0.0001431268 \\
\hline \multirow{6}{*}{$y_{7}$} & $a 5$ & 1 & 1 & 0 & 0 & 1 & 1 & 0 & 1 \\
\hline & $a 4$ & 1.3572130005 & 1.8097388978 & 1 & 1 & 5.3083619025 & 5.0581347472 & 1 & 2.0503248828 \\
\hline & $a 3$ & 1.2342340899 & 3.8604394101 & 5.8838370829 & 2.2562370610 & 10.3018254782 & 9.6996882654 & 4.7959686546 & 6.8378238706 \\
\hline & $a 2$ & 0.1577333624 & 2.9585351944 & 15.1889006110 & 0.2490035716 & 6.3185649178 & 5.3682685821 & 9.4236350956 & 1.3305939516 \\
\hline & $a 1$ & 0.0003662304 & 1.6304808086 & 12.2535482201 & 0.0000581560 & 0.7981747255 & 0.5349731362 & 5.1716543055 & 0.0515429933 \\
\hline & $a 0$ & 0.0000466952 & 0.1847430953 & 1.3430591345 & 0.0000064175 & 0.0167372794 & 0.0014379936 & 0.4822115293 & 0.0025696256 \\
\hline \multirow{6}{*}{$y_{8}$} & $a 5$ & 1 & 1 & 1 & 1 & 1 & 1 & 1 & 1 \\
\hline & $a 4$ & 0.1503165495 & 5.2958977956 & 1.4426653376 & 0.0819735316 & 1.6707276449 & 0.7143893521 & 0.7118270698 & 0.6364857458 \\
\hline & $a 3$ & 0.0060416343 & 9.6051772750 & 1.9036848539 & 0.0189506739 & 1.2823644598 & 0.1869629710 & 0.1427399489 & 0.0773446327 \\
\hline & $a 2$ & 0.0003013495 & 12.4041520034 & 0.8948105947 & 0.0002445433 & 0.1213568826 & 0.0126794867 & 0.0091525279 & 0.0029083717 \\
\hline & $a l$ & 0.0000071973 & 7.1742903199 & 0.0968367840 & 0.0000471779 & 0.0021935868 & 0.0003410102 & 0.0003667913 & 0.0002271625 \\
\hline & $a 0$ & 0.0000000114 & 0.7023874291 & 0.0021394427 & 0.0000000076 & 0.0000103854 & 0.0000209327 & 0.0000190806 & 0.0000029419 \\
\hline \multirow{6}{*}{$y_{9}$} & $a 5$ & 0 & 1 & 1 & 0 & 0 & 0 & 1 & 0 \\
\hline & a4 & 0 & 1.0520707432 & 0.0846599356 & 0 & 0 & 1 & 0.4973705586 & 1 \\
\hline & $a 3$ & 0 & 0.2011216030 & 0.0059078738 & I & 0 & 0.1004218807 & 0.0404825688 & 0.0696313712 \\
\hline & $a 2$ & 0 & 0.0135481797 & 0.0003194767 & 0.0682934679 & 1 & 0.0011761851 & 0.0006461549 & 0.0028875427 \\
\hline & $a l$ & 1 & 0.0005700614 & 0.0000081714 & 0.0126378766 & 0.1034187624 & 0.0000257065 & 0.0000139432 & 0.0001277360 \\
\hline & $a 0$ & 0.0012442849 & 0.0000067138 & 0.0000000235 & 0.0000088941 & 0.0011842736 & 0.0000002356 & 0.0000001644 & 0.0000015950 \\
\hline \multirow{6}{*}{$y_{10}$} & $a 5$ & 1 & 0 & 1 & 1 & 0 & 0 & 1 & 1 \\
\hline & a4 & 0.3508745334 & 1 & 10.3303617886 & 0.0266592523 & 1 & 1 & 12.1942436420 & 3.3101140498 \\
\hline & $a 3$ & 0.0329630540 & 1.8072304391 & 1.3849122268 & 0.0067533245 & 2.2582640232 & 10.1444276417 & 4.0762547364 & 7.3266801993 \\
\hline & $a 2$ & 0.0004869378 & 2.0463462435 & 0.3502970090 & 0.0000904611 & 1.7336864338 & 1.4145982715 & 0.2805338782 & 0.9991431874 \\
\hline & $a l$ & 0.0000342696 & 0.5705451687 & 0.0424946191 & 0.0000093553 & 0.2180957901 & 0.0213939878 & 0.0020121829 & 0.0420559587 \\
\hline & $a 0$ & 0.0000000216 & 0.0371332563 & 0.0000018047 & 0.0000000323 & 0.0050649862 & 0.0015783916 & 0.0000766420 & 0.0017131294 \\
\hline \multirow{6}{*}{$y_{H I}$} & $a 5$ & 0 & 1 & 0 & 1 & $l$ & 1 & 0 & 0 \\
\hline & $a 4$ & 0 & 0.8374120844 & 1 & 0.0205744522 & 0.1556860330 & 1.7553458934 & 1 & 1 \\
\hline & $a 3$ & 0 & 0.1807903308 & 0.1616483189 & 0.0041432406 & 0.0189629132 & 1.9200749947 & 0.2257513582 & 0.1612929715 \\
\hline & $a 2$ & 1 & 0.0083958996 & 0.7696995569 & 0.0000493362 & 0.0021255042 & 0.3222545834 & 0.0088047129 & 0.0073685733 \\
\hline & $a l$ & 0.0022672061 & 0.0003959719 & 0.1208917214 & 0.0000030674 & 0.0000638827 & 0.0060404997 & 0.0000904162 & 0.0005383537 \\
\hline & $a 0$ & 0.0000110931 & 0.0000057115 & 0.0018330126 & 0.0000000167 & 0.0000003994 & 0.0000226508 & 0.0000000207 & 0.0000069499 \\
\hline
\end{tabular}


Table 30 - Plant 2 - Sub-model 2 - Transfer Function Numerator Parameters.

\begin{tabular}{|c|c|c|c|c|c|c|c|c|c|}
\hline$P I$ & $N$ & $u_{I}$ & $u_{2}$ & $u_{3}$ & $u_{4}$ & $u_{5}$ & $u_{6}$ & $u_{7}$ & $d_{I}$ \\
\hline \multirow{6}{*}{$y_{l}$} & $b 5$ & 0 & 0 & 0 & 0 & 0 & 0 & 0 & 0.0000083959 \\
\hline & $b 4$ & 0 & 0 & 0 & 0 & 0.0001646988 & 0.0001945853 & 0 & 0.0000245339 \\
\hline & $b 3$ & 0 & 0.0004579769 & -0.0000755727 & 0 & -0.0000415313 & -0.0000363037 & 0 & -0.0000077387 \\
\hline & $b 2$ & 0.0000266875 & -0.0000120663 & -0.0000852804 & 0.0013504814 & 0.0000027681 & 0.0000025809 & 0.0004399067 & -0.0000030015 \\
\hline & $b l$ & 0.0000091466 & 0.0000010903 & 0.0000266649 & -0.0002071391 & -0.0000005413 & -0.0000003578 & -0.0002935855 & -0.0000000350 \\
\hline & bo & -0.0000000437 & -0.0000000313 & -0.0000000342 & 0.0000024410 & 04 & 0.0000000020 & -0.0000008720 & -0.0000000022 \\
\hline \multirow{6}{*}{$y_{2}$} & $b 5$ & 0 & 0 & 0 & 0 & 0.0000445325 & 0 & 0 & 0 \\
\hline & $b 4$ & 0 & 0 & 0 & 0 & 0.0002779238 & 0.0002916357 & 0 & 0.0003070780 \\
\hline & $b 3$ & 0.0010106250 & -0.0015726386 & 0 & 0 & -0.0001881113 & 0.0004773534 & 0.0001881203 & 0.0000832513 \\
\hline & $b 2$ & 0.0012054628 & 0.0028013185 & 0.0005213455 & -0.0190835358 & -0.0000545334 & -0.0003711473 & 0.0000794712 & -0.0001363461 \\
\hline & $b 1$ & 0.0002495583 & 0.0008950736 & 0.0001495288 & -0.0026425631 & -0.0000031566 & -0.0000514493 & -0.0001490255 & -0.0000003114 \\
\hline & $b 0$ & 0.0000004676 & 0.0000153172 & -0.0000000312 & -0.0000021044 & -0.0000000027 & -0.0000013860 & -0.0000004961 & -0.0000002835 \\
\hline \multirow{6}{*}{$y_{3}$} & $b 5$ & 0 & -0.0004267206 & 0 & 0 & 0 & 0 & 0 & 0 \\
\hline & $b 4$ & 0 & -0.0087536959 & 0 & 0 & 0.0001429595 & 0.0004666645 & 0.0001485433 & -0.0004235691 \\
\hline & $b 3$ & 0 & 0.0115364458 & -0.0045768795 & -0.0034364386 & 0.0012508687 & 0.0026747842 & 0.0009805454 & 0.0020048723 \\
\hline & $b 2$ & 0 & -0.0011981122 & 0.0059345573 & -0.0020279844 & -0.0011416812 & -0.0025076291 & -0.0009347483 & -0.0019532109 \\
\hline & $b 1$ & -0.0035877559 & 0.0000207564 & 0.0013661854 & -0.0000033794 & -0.0000266400 & -0.0000563681 & -0.0000240001 & -0.0001205239 \\
\hline & bo & 0.0064160140 & -0.0000040714 & 0.0000004825 & -0.0000017302 & -0.0000001717 & -0.0000003426 & -0.0000000903 & -0.0000033023 \\
\hline \multirow{6}{*}{$y_{4}$} & $b 5$ & 0 & -0.0004195370 & 0 & 0 & 0 & 0 & 0 & 0 \\
\hline & $b 4$ & 0 & -0.0072930566 & 0 & 0 & 0 & 0 & 0 & 0 \\
\hline & $b 3$ & 0 & 0.0083951082 & 0 & -0.0205209457 & 0 & 0 & 0 & 0 \\
\hline & $b 2$ & 0 & -0.0011352139 & -0.0146373318 & -0.0045843759 & 0 & 0 & 0 & -0.0008417440 \\
\hline & bl & -0.0045614631 & 0.0000023551 & 0.0163370273 & -0.0000060247 & -0.0010914736 & -0.0006197362 & -0.0009954657 & 0.0000003341 \\
\hline & bo & 0.0047473950 & -0.0000037782 & 0.0000209701 & -0.0000015374 & -0.0000004490 & -0.0000000668 & -0.0000005434 & -0.0000013480 \\
\hline \multirow{6}{*}{$y_{5}$} & $b 5$ & 0 & -0.0005394863 & 0 & 0 & 0 & 0 & 0 & 0 \\
\hline & $b 4$ & 0 & -0.0016683556 & 0 & 0 & 0 & 0 & 0 & 0 \\
\hline & $b 3$ & 0 & 0.0018487474 & 0 & -0.0031201381 & 0.0016208241 & 0.0003430117 & 0.0014969053 & 0 \\
\hline & $b 2$ & 0 & -0.0002780772 & 0 & -0.0011419668 & -0.0014593569 & -0.0005164719 & -0.0014175673 & 0.0007745400 \\
\hline & $b l$ & -0.0027651123 & -0.0000023218 & -0.0019280963 & -0.0000045661 & 0.0000013119 & 0.0000004274 & 0.0000013405 & -0.0009032513 \\
\hline & bo & 0.0017894571 & -0.0000009383 & 0.0011135411 & -0.0000003297 & -0.0000003316 & -0.0000001217 & -0.0000002536 & -0.0000028607 \\
\hline \multirow{6}{*}{$y_{6}$} & $b 5$ & 0 & 0 & -0.0000211806 & 0 & 0 & 0 & 0 & 0 \\
\hline & $b 4$ & 0 & -0.0015753530 & -0.0011157351 & 0 & 0 & -0.0000468704 & 0 & 0 \\
\hline & $b 3$ & 0 & 0.0023805120 & 0.0014797675 & -0.0070229353 & 0 & 0.0008365529 & 0 & 0.0005070473 \\
\hline & $b 2$ & 0.0037396289 & -0.0002208452 & 0.0000065378 & -0.0006138597 & 0.0008631190 & -0.0006965313 & 0.0005662017 & -0.0004538986 \\
\hline & $b l$ & 0.0001189712 & 0.0000042213 & 0.0000056547 & -0.0000056215 & -0.0012233663 & -0.0000090619 & -0.0004782242 & 0.0000013788 \\
\hline & bo & 0.0000014600 & -0.0000006401 & 02 & -0.0000004919 & -0.0000016283 & -0.0000000361 & -0.0000002515 & -0.0000010479 \\
\hline \multirow{6}{*}{$y_{7}$} & $b 5$ & -0.0061526846 & 0 & 0 & 0 & 0 & 0 & 0 & 0 \\
\hline & $b 4$ & 0.0296668498 & 0 & 0 & 0 & 0 & 0 & 0 & 0 \\
\hline & $b 3$ & 0.0008082933 & 0 & 0 & 0 & 0 & 0 & 0 & 0.2734867646 \\
\hline & $b 2$ & 0.0000636204 & 0.1321910413 & -4.2785460923 & -1.0846738670 & 0.5091044695 & 0.3581208846 & 0.4622904385 & 0.1156658241 \\
\hline & $b 1$ & 0.0000017696 & -0.0225098633 & -4.3044072055 & -1.9576299851 & -0.0203533212 & -0.0387203183 & 0.0129070084 & -0.0354414360 \\
\hline & bo & 07 & -0.0002223130 & -0.3355447832 & -0.2722545938 & -0.0417693541 & -0.0324541603 & -0.0314011268 & -0.0012892473 \\
\hline \multirow{6}{*}{$y_{8}$} & $b 5$ & 0 & 0 & 0 & 0.0000817090 & 0 & 0.0003981585 & 0 & 0 \\
\hline & $b 4$ & 0.0050271593 & -0.0055637836 & 0 & 0.0000344088 & 0.0148250023 & 0.0084741196 & 0 & 0 \\
\hline & $b 3$ & 0.0497708158 & 0.0147875692 & 0.0002901979 & -0.0000209241 & -0.0175359739 & -0.0100225475 & 0 & -0.0010680956 \\
\hline & $b 2$ & 0.0072378057 & -0.0036541940 & 0.0003623077 & -0.0000027475 & -0.0002701752 & -0.0005796065 & 0.0517751917 & -0.0064951298 \\
\hline & $b 1$ & 0.0002707512 & 0.0000315369 & 0.0000163354 & -0.0000004381 & -0.0000185236 & -0.0000148529 & -0.1099601828 & -0.0000563924 \\
\hline & bo & 0.0000007591 & -0.0000116269 & 0.0000062830 & -0.0000000115 & -0.0000001020 & -0.0000008944 & -0.0089639132 & -0.0000007683 \\
\hline \multirow{6}{*}{$y_{9}$} & $b 5$ & 0 & -0.0001188010 & 0 & 0 & 0 & 0.0012065733 & 0 & 0 \\
\hline & $b 4$ & 0 & -0.0240690388 & 0 & 0 & 0 & 0.0004719949 & 0 & 0 \\
\hline & $b 3$ & 0 & 0.0200082325 & 0 & 0 & 0 & -0.0004660992 & 0 & 0 \\
\hline & $b 2$ & -0.0002016495 & -0.0028511082 & 0 & -0.0000038731 & -0.0002153636 & -0.0000592822 & -0.0001977354 & -0.0002283097 \\
\hline & $b l$ & 0.0010289943 & 0.0000599742 & 0.0000166552 & -0.0000000435 & -0.0000460999 & -0.0000003429 & -0.0000350732 & -0.0000004358 \\
\hline & $b 0$ & 0.0013293070 & -0.0000107140 & -0.0000002102 & -06 & 0.0000000132 & -04 & 0.0000000127 & -0.0000003504 \\
\hline \multirow{6}{*}{$y_{I 0}$} & $b 5$ & -0.0040354413 & 0 & 0 & 0 & 0 & 0.0000614155 & 0.0000286214 & 0 \\
\hline & $b 4$ & 0.0322028014 & -0.0068020531 & 0 & 0.0000355112 & 0 & 0.0362719151 & 11.6760861163 & 0.0001643220 \\
\hline & $b 3$ & 0.0167444308 & 0.0239450464 & 0.0007411858 & 0.0025927586 & 0.3243103791 & 0.0019762885 & -2.8697500175 & 0.0240872290 \\
\hline & $b 2$ & 0.0011291981 & -0.0061656389 & 0.0004712910 & -0.0009114023 & 0.3059953575 & -0.0222968342 & -1.0800340567 & -0.0136841675 \\
\hline & $b 1$ & 0.0000027097 & -0.0000845970 & 0.0000043797 & -0.0000040963 & -0.3848958333 & -0.0011960293 & -0.0436156677 & -0.0035952072 \\
\hline & bo & 0.0000001239 & -0.0000294108 & 0.0000000318 & 0.0000001074 & -0.0292012080 & -0.0000163213 & -0.0000436047 & -0.0001918816 \\
\hline \multirow{6}{*}{$y_{I I}$} & $b 5$ & 0 & 0 & 0 & 0 & 0 & 0 & 0 & 0 \\
\hline & $b 4$ & 0 & 0.0000789961 & 0 & 0 & 0 & 0 & 0 & 0.0001827257 \\
\hline & $b 3$ & 0 & 0.0001541394 & 0.0000200309 & 0 & 0 & 0.0003744995 & 0 & -0.0002969679 \\
\hline & $b 2$ & 0 & -0.0000991345 & -0.0000001390 & 0 & 0 & -0.0004501734 & 0 & -0.0000682615 \\
\hline & $b l$ & 0.0012500148 & 0.0000001303 & 0.0000000686 & -0.0000089236 & -0.0005127704 & -0.0000065300 & -0.0005042485 & -0.0000001914 \\
\hline & bo & 0.0000008153 & -0.0000003078 & -05 & -0.0000000353 & 0.0000001098 & -0.0000000174 & -0.0000000136 & -0.0000000119 \\
\hline
\end{tabular}


Table 31 - Plant 2 - Sub-model 2 - Transfer Function Denominator Parameters.

\begin{tabular}{|c|c|c|c|c|c|c|c|c|c|}
\hline$P I$ & $D$ & $u_{I}$ & $u_{2}$ & $u_{3}$ & $u_{4}$ & $u_{5}$ & $u_{6}$ & $u_{7}$ & $d_{l}$ \\
\hline \multirow{6}{*}{$y_{l}$} & $a 5$ & 1 & 1 & 0 & 0 & 1 & 1 & 0 & 1 \\
\hline & $a 4$ & 0.9022658577 & 1.4167540291 & 0 & 0 & 0.1402274786 & 0.2397707429 & 1 & 0.3139954487 \\
\hline & $a_{3}$ & 0.3173851327 & 0.1144181768 & 1 & 0 & 0.0156038975 & 0.0114872846 & 21.6057345836 & 0.0276853766 \\
\hline & $a_{2}$ & 0.0255477291 & 0.0076090816 & 0.1329340032 & 1 & 0.0018798958 & 0.0021917784 & 2.5612733951 & 0.0008726327 \\
\hline & $a l$ & 0.0002253713 & 0.0002574887 & 0.0018370107 & 0.0017388599 & 0.0000294349 & 0.0000166053 & 0.0435563322 & 0.0000205078 \\
\hline & $a 0$ & 0.0000002662 & 0.0000066296 & 0 & 0.0000747768 & 0.0000000080 & 0.0000001465 & 0.0001119346 & 0.0000003009 \\
\hline \multirow{6}{*}{$y_{2}$} & $a 5$ & 0 & 0 & 1 & 1 & 1 & 0 & 0 & 0 \\
\hline & a4 & 1 & 1 & 1.4609958758 & 3.8032430285 & 0.5491104364 & 1 & 0 & 1 \\
\hline & $a 3$ & 0.4897330091 & 1.0653609121 & 0.9353149427 & 13.3563647566 & 0.0825374409 & 0.3531747536 & 1 & 0.1732826433 \\
\hline & $a 2$ & 0.0563708683 & 0.3286032327 & 0.1610617195 & 2.0530141748 & 0.0042803077 & 0.0346824855 & 0.2093974551 & 0.0053417270 \\
\hline & $a l$ & 0.0006991356 & 0.0112487072 & 0.0023945335 & 0.0328002825 & 0.0000540074 & 0.0010997108 & 0.0035888443 & 0.0003592680 \\
\hline & $a 0$ & 0.0000071343 & 0.0000907810 & 0.0000000301 & 0.0000197011 & 0.0000000388 & 0.0000113428 & 0.0000142317 & 0.0000053242 \\
\hline \multirow{6}{*}{$y_{3}$} & $a 5$ & 0 & 1 & 1 & 0 & 0 & 0 & 0 & 0 \\
\hline & a4 & 0 & 1.1493784228 & 3.8886158584 & 1 & 1 & 1 & 1 & 1 \\
\hline & $a 3$ & 0 & 0.3309695649 & 2.2749239139 & 0.3922966508 & 0.3363296518 & 0.3252537675 & 0.3434637049 & 0.4009238735 \\
\hline & $a^{2}$ & 1 & 0.0104316017 & 0.2865644933 & 0.0070564978 & 0.0114593500 & 0.0108665445 & 0.0122610604 & 0.0251294677 \\
\hline & $a l$ & 0.3449236252 & 0.0010461294 & 0.0042584574 & 0.0003343929 & 0.0001576989 & 0.0001448445 & 0.0001545607 & 0.0008157440 \\
\hline & $a 0$ & 0.0048397043 & 0.0000129245 & 0.0000023299 & 0.0000052884 & 0.0000006354 & 0.0000005570 & 0.0000003900 & 0.0000074581 \\
\hline \multirow{6}{*}{$y_{4}$} & $a 5$ & 0 & 1 & 0 & 1 & 1 & 1 & 1 & 1 \\
\hline & $a 4$ & 0 & 1.0201588062 & 1 & 3.5714659176 & 0.7507242908 & 0.6735141682 & 0.8286698481 & 1.3977570748 \\
\hline & $a 3$ & 0 & 0.1800965935 & 12.2595753515 & 0.5535729355 & 0.8632976847 & 0.7621399370 & 0.9532127408 & 0.1874560514 \\
\hline & $a 2$ & 1 & 0.0072207071 & 1.9553151965 & 0.0088355212 & 0.1183576305 & 0.1026561292 & 0.1330434304 & 0.0049621452 \\
\hline & $a l$ & 0.2091556904 & 0.0005193761 & 0.0313827594 & 0.0001892288 & 0.0017253097 & 0.0014724350 & 0.0019524728 & 0.0002989867 \\
\hline & $a 0$ & 0.0026829128 & 0.0000057891 & 0.0000371099 & 0.0000026043 & 0.0000017616 & 0.0000009751 & 0.0000023834 & 0.0000043654 \\
\hline \multirow{6}{*}{$y_{5}$} & $a 5$ & 0 & 1 & 0 & 0 & 1 & 1 & 1 & 1 \\
\hline & $a 4$ & 0 & 0.5687713341 & 0 & 1 & 1.4699109337 & 0.8346571866 & 1.7451058141 & 1.3745938232 \\
\hline & $a 3$ & 0 & 0.0801955458 & 0 & 0.1270054779 & 0.1624665830 & 0.0893185832 & 0.1919656249 & 2.0351323311 \\
\hline & $a^{2}$ & 1 & 0.0034060145 & 1 & 0.0024742197 & 0.0023563156 & 0.0012771814 & 0.0027367182 & 0.2143254141 \\
\hline & $a l$ & 0.1579902477 & 0.0002111567 & 0.1257493303 & 0.0000417821 & 0.0000380656 & 0.0000217722 & 0.0000348602 & 0.0035846856 \\
\hline & a0 & 0.0018123064 & 0.0000019125 & 0.0017827840 & 0.0000005723 & 0.0000004720 & 0.0000002623 & 0.0000004398 & 0.0000099388 \\
\hline \multirow{6}{*}{$y_{6}$} & $a 5$ & 0 & 1 & 1 & 1 & 1 & 0 & 0 & 0 \\
\hline & $a 4$ & 1 & 0.9698714976 & 0.5954991382 & 2.7477883929 & 1.3258623878 & 1 & 0 & 1 \\
\hline & $a 3$ & 1.0553524202 & 0.3615643339 & 0.0180458683 & 0.2882374723 & 2.5555033053 & 0.4453838244 & 1 & 0.3692358911 \\
\hline & $a_{2}$ & 0.0437484849 & 0.0105674664 & 0.0023318739 & 0.0061063638 & 0.9265907561 & 0.0122280387 & 0.4491529310 & 0.0083144703 \\
\hline & $a l$ & 0.0009137417 & 0.0010295630 & 0.0000377092 & 0.0002323759 & 0.0156587066 & 0.0001133455 & 0.0073182757 & 0.0008544210 \\
\hline & $a 0$ & 0.0000024354 & 0.0000143565 & 0.0000000180 & 0.0000031408 & 0.0000252732 & 0.0000003001 & 0.0000069820 & 0.0000138852 \\
\hline \multirow{6}{*}{$y_{7}$} & $a 5$ & 1 & 1 & 0 & 1 & 0 & 0 & 0 & 1 \\
\hline & $a 4$ & 0.2516606967 & 3.0552345950 & 1 & 4.7678810673 & 1 & 1 & 1 & 4.4033571585 \\
\hline & $a 3$ & 0.0075797767 & 3.3615651340 & 5.5873022701 & 17.9036346473 & 4.5314974527 & 4.6258713970 & 4.5222249435 & 10.1203516285 \\
\hline & $a 2$ & 0.0005472978 & 2.8085427909 & 14.2614550266 & 37.2565227113 & 8.9445384355 & 9.0630227573 & 9.0057115879 & 8.9918088444 \\
\hline & $a l$ & 0.0000115863 & 0.4171279077 & 11.5592086229 & 29.3734958867 & 5.1306521332 & 5.0596839112 & 5.2583191917 & 1.4625895061 \\
\hline & $a 0$ & 0.0000000266 & 0.0037049659 & 1.2631255000 & 2.6897968259 & 0.5015135716 & 0.4870742807 & 0.5131920208 & 0.0371892621 \\
\hline \multirow{6}{*}{$y_{8}$} & $a 5$ & 1 & 1 & 1 & 1 & 1 & 1 & 1 & 1 \\
\hline & a4 & 2.9875467844 & 1.3903808122 & 0.5582292966 & 0.4969747432 & 1.9834821136 & 1.8095909167 & 6.3309963745 & 1.7812822383 \\
\hline & $a 3$ & 0.7203395005 & 1.0577258493 & 1.2018544174 & 0.0783270383 & 0.2571980245 & 0.2945232188 & 14.3930697228 & 1.5055856751 \\
\hline & $a^{2}$ & 0.0600511505 & 0.1210196845 & 0.1666989405 & 0.0098272301 & 0.0056938940 & 0.0137248879 & 17.4986753520 & 0.1692941205 \\
\hline & $a l$ & 0.0017076050 & 0.0038189527 & 0.0233805869 & 0.0005700999 & 0.0002428236 & 0.0004345511 & 3.2100470531 & 0.0013953628 \\
\hline & $a 0$ & 0.0000030964 & 0.0003553788 & 0.0021261344 & 0.0000118567 & 0.0000013145 & 0.0000163452 & 0.1418164524 & 0.0000203861 \\
\hline \multirow{6}{*}{$y_{9}$} & $a 5$ & 0 & 1 & 0 & 0 & 1 & 1 & 1 & 0 \\
\hline & a4 & 0 & 32.6992877304 & 0 & 1 & 1.2628323939 & 0.2147019182 & 1.4360957771 & 1 \\
\hline & $a 3$ & 0 & 4.7137164960 & 1 & 0.0818784103 & 0.3226717796 & 0.0132708183 & 0.3421977710 & 0.1041241910 \\
\hline & $a 2$ & 1 & 0.2033522271 & 0.1246992002 & 0.0019432189 & 0.0225012340 & 0.0002018663 & 0.0227273771 & 0.0026059392 \\
\hline & $a l$ & 0.1386706609 & 0.0172574502 & 0.0000159029 & 0.0000193616 & 0.0002415064 & 0.0000013049 & 0.0002427179 & 0.0001606628 \\
\hline & $a 0$ & 0.0012259182 & 0.0002098152 & 0.0000019830 & 0.0000000088 & 0.0000008700 & 0.0000000016 & 0.0000007680 & 0.0000016400 \\
\hline \multirow{6}{*}{$y_{10}$} & $a 5$ & 1 & 1 & 1 & 0 & 1 & 1 & 1 & 0 \\
\hline & $a 4$ & 0.6778925912 & 1.2489268998 & 0.4669077588 & 1 & 10.1678115424 & 2.8874563752 & 456.6826204285 & 1 \\
\hline & $a 3$ & 0.1034473339 & 1.5270514898 & 1.5154201059 & 5.6154548676 & 26.7957935483 & 2.4961804940 & 178.6062121511 & 2.7366984250 \\
\hline & $a 2$ & 0.0044574198 & 0.2505126585 & 0.1507556211 & 0.9081818579 & 32.8319818511 & 0.3885616676 & 18.4290042430 & 0.8939480133 \\
\hline & $a l$ & 0.0000142108 & 0.0122712014 & 0.0014157508 & 0.0001173394 & 6.1339980248 & 0.0145190679 & 0.5019425324 & 0.0952741995 \\
\hline & $a 0$ & 0.0000005982 & 0.0008566492 & 0.0000002274 & 0.0000153144 & 0.2456834863 & 0.0001769231 & 0.0004975308 & 0.0031165035 \\
\hline \multirow{6}{*}{$y_{11}$} & $a 5$ & 1 & 1 & 1 & 0 & 1 & 0 & 1 & 1 \\
\hline & a4 & 0.9049849117 & 0.4975606977 & 0.1427663052 & 0 & 1.2602621676 & 1 & 1.5926543722 & 0.3722848156 \\
\hline & $a 3$ & 0.6372676372 & 0.0997878349 & 0.0046206328 & 1 & 1.6580641686 & 0.1926480226 & 2.1277577405 & 0.0373841108 \\
\hline & $a 2$ & 0.0849876385 & 0.0028738145 & 0.0005153596 & 0.1211725019 & 0.2558788680 & 0.0051261064 & 0.3310813409 & 0.0006396440 \\
\hline & al & 0.0013767782 & 0.0003055812 & 0.0000032551 & 0.0024834831 & 0.0037105051 & 0.0000466205 & 0.0048703332 & 0.0000075642 \\
\hline & $a 0$ & 0.0000040080 & 0.0000039898 & 0.0000000085 & 0.0000015873 & 0.0000007325 & 0.0000000730 & 0.0000022506 & 0.0000000846 \\
\hline
\end{tabular}


Table 32 - Plant 2 - Sub-model 3 - Transfer Function Numerator Parameters.

\begin{tabular}{|c|c|c|c|c|c|c|c|c|c|}
\hline$P I$ & $N$ & $u_{I}$ & $u_{2}$ & $u_{3}$ & $u_{4}$ & $u_{5}$ & $u_{6}$ & $u_{7}$ & $d_{l}$ \\
\hline \multirow{6}{*}{$y_{l}$} & $b 5$ & 0 & 0 & 0 & 0 & 0 & 0 & 0 & 0 \\
\hline & $b 4$ & 0 & 0 & 0 & 0 & 0 & 0 & 0 & 0 \\
\hline & $b 3$ & 0 & 0.0000175239 & 0 & 0 & -0.0000333074 & -0.0000372682 & 0.0000319584 & 0 \\
\hline & $b 2$ & 0 & 0.0000005680 & 0 & 0 & -0.0000392829 & -0.0000228452 & -0.0000190525 & 0.0010899294 \\
\hline & $b 1$ & 0.0000665099 & 0.0000000178 & 0.0001262609 & 0.0000331833 & -0.0000097290 & -0.0000009110 & -0.0000011602 & -0.0002110223 \\
\hline & bo & -0.0000005132 & -05 & 0.0000175729 & -0.0000002939 & -0.0000000461 & -0.0000000067 & 0.0000000017 & 0.0000013831 \\
\hline \multirow{6}{*}{$y_{2}$} & $b 5$ & 0 & 0 & 0 & 0 & 0 & 0 & 0 & 0 \\
\hline & b4 & 0 & 0 & 0 & 0 & 0 & 0 & 0 & 0 \\
\hline & $b 3$ & -0.0016103813 & 0.0004405647 & 0 & 0 & 0 & 0 & 0 & -0.0003027473 \\
\hline & $b 2$ & 0.0019083898 & -0.0000036305 & 0.0004840073 & 0.0058592297 & 0 & -0.0008393909 & 0 & -0.0000034523 \\
\hline & $b 1$ & 0.0000090411 & 0.0000016609 & 0.0000071425 & -0.0000074492 & -0.0003246591 & -0.0002703587 & -0.0001866340 & -0.0000047599 \\
\hline & bo & -0.0000000990 & -0.0000000097 & 0.0000000210 & 0.0000000261 & -0.0000004223 & -0.0000008478 & 0.0000006028 & 0.0000000048 \\
\hline \multirow{6}{*}{$y_{3}$} & $b 5$ & 0 & 0 & 0 & 0 & 0 & 0 & 0 & 0 \\
\hline & $b 4$ & 0 & 0 & 0 & 0 & 0 & 0 & 0 & -0.0002043482 \\
\hline & $b 3$ & 0 & 0 & 0.0052484237 & 0 & 0 & 0 & 0 & -0.0022874121 \\
\hline & $b 2$ & 0.0061340698 & 0 & -0.0095451519 & 0 & 0 & 0 & 0 & -0.0010612134 \\
\hline & $b l$ & 0.0000140237 & -0.0000801431 & 0.0061282710 & 0.0089135247 & -0.0021110274 & -0.0020838636 & -0.0047037902 & -0.0000057284 \\
\hline & bo & -0.0000001858 & -0.0000004924 & -0.0000033103 & 0.0051078944 & 0.0000005953 & -0.0000009606 & -0.0000003227 & -0.0000000687 \\
\hline \multirow{6}{*}{$y_{4}$} & $b 5$ & 0 & 0 & 0 & 0 & 0 & 0 & 0 & 0.0008202328 \\
\hline & $b 4$ & -0.0030490538 & 0 & 0 & 0 & -0.0002678044 & 0 & 0 & 0.0001039400 \\
\hline & $b 3$ & -0.0030681869 & 0 & 0 & 0 & -0.0014278452 & 0 & 0 & -0.0007550896 \\
\hline & $b 2$ & 0.0029098675 & -0.0000196715 & 0.0030428617 & 0 & -0.0000045963 & 0 & 0 & -0.0000441384 \\
\hline & $b 1$ & 0.0000046170 & -0.0000016641 & 0.0055855208 & 0.0076101457 & -0.0000020413 & -0.0009716500 & -0.0011924207 & -0.0000042160 \\
\hline & bo & -0.0000000679 & -0.0000000313 & 0.0001668090 & -0.0000106677 & -0.0000000064 & -0.0000005271 & 0.0000003736 & -0.0000000104 \\
\hline \multirow{6}{*}{$y_{5}$} & $b 5$ & 0 & 0 & 0 & 0 & 0 & 0 & -0.0002883790 & 0 \\
\hline & $b 4$ & 0 & 0 & 0 & 0 & 0.0023714099 & 0.0027427250 & 0.0009104693 & 0 \\
\hline & $b 3$ & 0 & 0 & 0 & 0.0012924335 & -0.0013684009 & -0.0015221134 & -0.0006148573 & 0.0017498474 \\
\hline & $b 2$ & -0.0156977637 & 0.0017547171 & 0.0026802565 & 0.0006677549 & 0.0000100803 & -0.0000762867 & -0.0000342308 & -0.0007545139 \\
\hline & $b l$ & 0.0007754898 & -0.0003634464 & 0.0000078206 & 0.0000115395 & -0.0000047640 & -0.0000047937 & -0.0000039121 & 0.0000023857 \\
\hline & bo & 0.0029331458 & -0.0000018932 & 0.0000000138 & -0.0000000302 & 0.0000000017 & 0.0000000020 & -0.0000000012 & -0.0000001466 \\
\hline \multirow{6}{*}{$y_{6}$} & $b 5$ & 0 & 0 & 0 & 0 & 0 & 0 & 0 & 0 \\
\hline & $b 4$ & 0 & 0 & 0 & 0 & 0 & 0 & 0 & 0 \\
\hline & $b 3$ & 0 & 0 & 0 & 0 & 0 & 0 & 0.0002852926 & 0 \\
\hline & $b 2$ & 0.0033199036 & 0.0015373411 & -0.0035800828 & 0.0004254101 & 0 & 0 & 0.0001025960 & 0 \\
\hline & $b l$ & 0.0001184041 & -0.0001885723 & 0.0020361844 & 0.0000226659 & -0.0007031823 & -0.0008220264 & -0.0003432217 & -0.0007390235 \\
\hline & bo & -0.0000003228 & 0.0000005544 & 0.0000024102 & -0.0000003319 & -0.0000000609 & -0.0000000370 & -0.0000002025 & 0.0000023095 \\
\hline \multirow{6}{*}{$y_{7}$} & $b 5$ & 0 & 0 & 0 & 0 & 0 & 0 & 0 & 0 \\
\hline & $b 4$ & 0 & 0 & 0 & 0 & 0 & 0 & 0 & 0 \\
\hline & b3 & 0.0970009299 & 0 & 0 & 0 & 0 & 0.7354469313 & 0.2528129462 & 0 \\
\hline & $b 2$ & 0.0422178516 & 0.0224736172 & -1.9154960249 & 0 & 1.2772605815 & 0.0526000159 & 0.0260166174 & 0.5305425938 \\
\hline & $b 1$ & 0.0000453325 & 0.0277576844 & -1.9601720792 & 25.3117557456 & -0.0605915309 & -0.1091498343 & -0.0239855565 & 0.2047934264 \\
\hline & bo & 0.0000115303 & -0.0066741618 & -0.1568666168 & 5.3411632263 & -0.1313758473 & -0.0060268188 & -0.0005026206 & -0.0859792667 \\
\hline \multirow{6}{*}{$y_{8}$} & $b 5$ & 0 & 0 & 0 & 0 & 0 & -0.0083261013 & 0 & 0 \\
\hline & $b 4$ & 0.0718807243 & 0 & 0 & 0.0000748122 & 0 & 0.6090570143 & 0.1162237335 & 0 \\
\hline & b3 & 0.1855911945 & 0.0064947786 & 0.0023654778 & 0.0000211687 & 0 & -0.6757156899 & -0.1278380390 & -0.0296744462 \\
\hline & $b 2$ & 0.0248093522 & 0.0152618232 & 0.0000120402 & 0.0000002983 & 0.0693444306 & 0.0002008011 & -0.0003207655 & -0.0183699815 \\
\hline & $b 1$ & 0.0001961484 & -0.0073695112 & 0.0000080242 & 0.0000000788 & -0.1007077374 & -0.0016791518 & -0.0004219872 & -0.0004884064 \\
\hline & bo & 0.0000019400 & -0.0000717456 & -0.0000000079 & 02 & -0.0000599623 & -0.0000019481 & -0.0000017881 & -0.0000229578 \\
\hline \multirow{6}{*}{$y_{9}$} & $b 5$ & 0 & 0 & 0 & 0 & 0 & 0 & 0 & 0 \\
\hline & $b 4$ & 0 & 0 & 0 & 0 & 0 & 0 & 0 & 0 \\
\hline & $b 3$ & 0 & 0 & 0 & 0 & 0 & -0.0001405224 & 0 & 0 \\
\hline & $b 2$ & -0.0275423287 & 0.0005879621 & 0.0000007831 & 0.0000000252 & 0 & -0.0000556917 & 0 & 0 \\
\hline & $b l$ & 0.0047213447 & -0.0000178545 & 0.0000000051 & -0.0000000418 & -0.0002381400 & -0.0000034266 & -0.0001410354 & -0.0001006227 \\
\hline & bo & -0.0000441181 & 0.0000017793 & 04 & 0.0000000012 & 0.0000010185 & 0.0000000137 & 0.0000002612 & 0.0000008231 \\
\hline \multirow{6}{*}{$y_{I 0}$} & $b 5$ & 0 & -0.0000594176 & 0 & 0 & 0 & 0.0002954625 & 0 & 0 \\
\hline & $b 4$ & 0.0664476649 & -0.0013910804 & -0.0023953517 & 0 & 0 & 0.0629222633 & 0.0000711968 & 0 \\
\hline & $b 3$ & 0.2247552834 & 0.0045989169 & 0.0023891502 & -0.0042855275 & 0.2203472006 & -0.0070594978 & 0.0294013806 & 0.0348918527 \\
\hline & $b 2$ & 0.0016409563 & 0.0027479811 & 0.0008943826 & 0.0012466645 & 0.1562178688 & -0.0298472639 & 0.0002873347 & -0.0086190178 \\
\hline & $b 1$ & 0.0001930643 & -0.0014772641 & 0.0005389002 & 0.0000429309 & -0.2299438601 & -0.0014515736 & -0.0084886602 & -0.0128737798 \\
\hline & bo & 0.0000010602 & -0.0004231430 & -0.0000000678 & 0.0000000986 & -0.0165988601 & -0.0000014236 & -0.0004700969 & -0.0006437994 \\
\hline \multirow{6}{*}{$y_{I I}$} & $b 5$ & 0 & 0 & 0 & 0 & 0 & 0 & 0 & 0 \\
\hline & $b 4$ & 0 & 0 & 0 & 0 & 0 & 0 & 0 & 0 \\
\hline & b3 & 0 & 0 & 0 & -0.0001029896 & 0 & 0 & 0 & -0.0005288331 \\
\hline & $b 2$ & 0.0027001630 & 0.0014687961 & 0 & -0.0000041810 & 0 & 0 & 0.0048142306 & -0.0000023269 \\
\hline & $b 1$ & 0.0000076554 & -0.0003705849 & 01 & 0.0000014123 & -0.0004753838 & -0.0013369067 & -0.0012253774 & -0.0000019404 \\
\hline & bo & -0.0000000454 & 0.0000002433 & 0 & -0.0000000094 & -0.0000002590 & 0.0000002482 & 0.0000115119 & -0.0000000017 \\
\hline
\end{tabular}


Table 33 - Plant 2 - Sub-model 3 - Transfer Function Denominator Parameters.

\begin{tabular}{|c|c|c|c|c|c|c|c|c|c|}
\hline$P I$ & $D$ & $u_{l}$ & $u_{2}$ & $u_{3}$ & $u_{4}$ & $u_{5}$ & $u_{6}$ & $u_{7}$ & $d_{I}$ \\
\hline \multirow{6}{*}{$y_{I}$} & $a 5$ & 1 & 1 & 0 & 0 & 0 & 1 & 0 & 0 \\
\hline & a4 & 1.9437103443 & 1.0792119713 & 0 & 1 & 1 & 0.9072922849 & 1 & 0 \\
\hline & $a 3$ & 0.7143443581 & 0.0707527664 & 0 & 2.1983888194 & 0.4251510121 & 0.1091559238 & 0.2020622332 & 0 \\
\hline & $a 2$ & 0.0859927770 & 0.0078773966 & 1 & 0.5297253220 & 0.0329832869 & 0.0045115186 & 0.0102481912 & 1 \\
\hline & al & 0.0004979633 & 0.0000061654 & 0.1356894124 & 0.0031492844 & 0.0006812622 & 0.0000754165 & 0.0001052777 & 0.0036092864 \\
\hline & $a 0$ & 0.0000009085 & 0.0000006791 & 0.0011941802 & 0.0000088090 & 0 & 0 & 0.0000001004 & 0.0000418789 \\
\hline \multirow{6}{*}{$y_{2}$} & $a 5$ & 0 & 0 & 1 & 0 & 0 & 0 & 0 & 1 \\
\hline & a4 & 1 & 1 & 2.1075204692 & 1 & 0 & 0 & 0 & 0.1975822494 \\
\hline & $a 3$ & 0.2564361342 & 0.0021601916 & 0.7658630905 & 13.4277390131 & 1 & 1 & 1 & 0.0205439005 \\
\hline & $a 2$ & 0.0046579580 & 0.0044086048 & 0.0233969096 & 0.2240552976 & 0.1828531210 & 0.1123260894 & 0.2374377036 & 0.0029399411 \\
\hline & $a l$ & 0.0000207119 & 0.0000091743 & 0.0002014657 & 0.0000647941 & 0.0030779313 & 0.0021939279 & 0.0023543267 & 0.0000393287 \\
\hline & $a 0$ & 0.0000000554 & 0.0000006354 & 0.0000002153 & 0.0000010811 & 0 & 0 & 0.0000046793 & 0.0000000188 \\
\hline \multirow{6}{*}{$y_{3}$} & $a 5$ & 0 & 0 & 0 & 0 & 0 & 0 & 0 & 1 \\
\hline & a4 & 1 & 0 & 0 & 0 & 0 & 0 & 1 & 0.9925006063 \\
\hline & $a 3$ & 0.2154916885 & 1 & 1 & 0 & 1 & 1 & 6.5221993089 & 0.1425551858 \\
\hline & $a 2$ & 0.0038821826 & 0.0824912314 & 0.5471030839 & 0 & 0.2177880861 & 0.2189989745 & 1.2682328782 & 0.0030819964 \\
\hline & al & 0.0000073457 & 0.0020456550 & 0.0081355739 & 1 & 0.0032679128 & 0.0034723635 & 0.0201941630 & 0.0000197278 \\
\hline & $a 0$ & 0.0000001312 & 0.0000006134 & 0.0000013831 & 0.0134701790 & 0.0000013529 & 0.0000018736 & 0.0000021755 & 0.0000001494 \\
\hline \multirow{6}{*}{$y_{4}$} & $a 5$ & 0 & 1 & 0 & 1 & 1 & 0 & 0 & 1 \\
\hline & a4 & 1 & 0.2702288864 & 1 & 0.5666471409 & 0.1417926749 & 0 & 1 & 0.1952332486 \\
\hline & $a 3$ & 0.1535808615 & 0.0430988270 & 1.8948298151 & 1.9893123147 & 0.0040484736 & 1 & 0.9194209174 & 0.0158637915 \\
\hline & $a 2$ & 0.0025558601 & 0.0017667511 & 0.3679234928 & 0.8345452862 & 0.0002071486 & 0.1310004623 & 0.1310771296 & 0.0008773493 \\
\hline & $a l$ & 0.0000049981 & 0.0000647373 & 0.0132345705 & 0.0115816918 & 0.0000037175 & 0.0020809290 & 0.0018886121 & 0.0000138640 \\
\hline & $a 0$ & 0.0000000815 & 0.0000000308 & 0.0001292340 & 0.0000031502 & 0.0000000095 & 0 & 0.0000003708 & 0 \\
\hline \multirow{6}{*}{$y_{5}$} & $a 5$ & 0 & 0 & $I$ & 0 & 1 & $I$ & 1 & 0 \\
\hline & $a 4$ & 0 & 1 & 1.0808078268 & $I$ & 0.1356875409 & 0.1658560715 & 0.1905169869 & 1 \\
\hline & $a 3$ & 0 & 1.3450114802 & 0.1294062948 & 0.1325142579 & 0.0053589168 & 0.0110564208 & 0.0156341499 & 0.1405678430 \\
\hline & $a 2$ & 1 & 0.1387174401 & 0.0022962383 & 0.0041750403 & 0.0004739352 & 0.0004350384 & 0.0008724358 & 0.0017794477 \\
\hline & $a 1$ & 0.1578057914 & 0.0030303748 & 0.0000010316 & 0.0000233801 & 0.0000065632 & 0.0000052534 & 0.0000115330 & 0.0000303582 \\
\hline & $a 0$ & 0.0017228853 & 0.0000027633 & 0.0000000182 & 0.0000000597 & 0.0000000135 & 0.0000000063 & 0 & 0.0000003377 \\
\hline \multirow{6}{*}{$y_{6}$} & $a 5$ & 1 & 0 & 0 & 0 & 1 & 0 & 0 & 0 \\
\hline & a4 & 1.2633682283 & 0 & 0 & 1 & 1.0862546737 & 0 & 0 & 0 \\
\hline & $a 3$ & 0.4565080131 & 0 & 0 & 0.7281657003 & 1.1533039922 & $I$ & 1 & 1 \\
\hline & $a 2$ & 0.0215590591 & 1 & 1 & 0.0717378832 & 0.3623527309 & 0.3105109927 & 0.3582234572 & 0.3952098298 \\
\hline & al & 0.0001887205 & 0.0110687674 & 0.0177371785 & 0.0000433089 & 0.0058685140 & 0.0049962541 & 0.0061996472 & 0.0043662938 \\
\hline & $a 0$ & 0.0000002882 & 0.0000139002 & 0.0000430692 & 0.0000008697 & 0.0000012371 & 0.0000012273 & 0 & 0.0000011461 \\
\hline \multirow{6}{*}{$y_{7}$} & $a 5$ & 1 & 1 & 0 & 1 & 1 & 1 & 1 & 0 \\
\hline & a4 & 1.3029538655 & 1.9091158389 & 1 & 32.1874512866 & 4.1088033657 & 5.7423226156 & 4.5673784461 & 1 \\
\hline & $a 3$ & 0.8604396958 & 4.2177240905 & 4.5948269484 & 187.1237466730 & 16.1937504630 & 11.2783989314 & 9.0509097484 & 4.3568320419 \\
\hline & $a 2$ & 0.0980359743 & 3.4071816312 & 11.2353745117 & 875.9302122263 & 25.6972487651 & 8.1087497083 & 5.8644876839 & 9.7378446459 \\
\hline & $a 1$ & 0.0003130028 & 2.1630282268 & 9.1694637496 & 1174.4912737547 & 14.7783594479 & 1.3136450563 & 0.7496808360 & 8.8452948280 \\
\hline & $a 0$ & 0.0000250928 & 0.2708406040 & 1.1310118916 & 126.9067017526 & 1.4978805877 & 0.0480188375 & 0.0127866078 & 1.1226297471 \\
\hline \multirow{6}{*}{$y_{8}$} & $a 5$ & 1 & 1 & 0 & 1 & 0 & 1 & 1 & 1 \\
\hline & $a 4$ & 1.0339842524 & 1.9127912654 & 1 & 0.2230116372 & 0 & 12.7467919109 & 7.2549735463 & 1.1970907055 \\
\hline & $a 3$ & 0.2284505932 & 2.2769193141 & 0.1170897031 & 0.0217619164 & 1 & 1.4410824244 & 0.8808461329 & 0.5890879389 \\
\hline & $a 2$ & 0.0139786303 & 1.2995303429 & 0.0044119139 & 0.0008039078 & 2.3675030916 & 0.0330596082 & 0.0272952806 & 0.0641150113 \\
\hline & al & 0.0001146625 & 0.1562441146 & 0.0003781282 & 0.0000628793 & 0.2714326500 & 0.0036443081 & 0.0028805275 & 0.0019656608 \\
\hline & $a 0$ & 0.0000009217 & 0.0013521820 & 0.0000000640 & 0.0000000852 & 0 & 0.0000017461 & 0.0000099237 & 0.0000629548 \\
\hline \multirow{6}{*}{$y_{9}$} & $a 5$ & 0 & 0 & $I$ & 1 & 0 & 1 & 0 & 0 \\
\hline & $a 4$ & 0 & 0 & 0.1953875742 & 0.6736834115 & 0 & 0.4000706693 & 0 & 0 \\
\hline & $a 3$ & 0 & 0 & 0.0078388615 & 0.2383293498 & 1 & 0.0553641531 & 1 & 1 \\
\hline & $a 2$ & 1 & 1 & 0.0002745032 & 0.1205854426 & 0.1118368532 & 0.0024845421 & 0.1069916161 & 0.1156748743 \\
\hline & $a l$ & 0.0000000751 & 04 & 0.0000036622 & 0.0018937306 & 0.0008161224 & 0.0000184722 & 0.0011263857 & 0.0003763260 \\
\hline & $a 0$ & 0.0000425295 & 0.0001452077 & 0.0000000042 & 0.0000002790 & 0.0000027146 & 0.0000000354 & 0.0000010150 & 0.0000048594 \\
\hline \multirow{6}{*}{$y_{10}$} & $a 5$ & 1 & 1 & 1 & 0 & 1 & 1 & 0 & 0 \\
\hline & a4 & 3.3902397213 & 1.8661201094 & 1.7012287890 & 1 & 7.6019118506 & 3.2652756085 & 1 & 1 \\
\hline & $a 3$ & 0.4043723931 & 2.5864514964 & 2.1031711520 & 14.9867882816 & 18.0062174622 & 2.5258945684 & 3.2326786915 & 2.5191818108 \\
\hline & $a 2$ & 0.0050261507 & 1.6423202143 & 1.2714686993 & 5.7470030424 & 21.4910426112 & 0.3688696063 & 2.2926086962 & 1.4945664037 \\
\hline & al & 0.0003415775 & 0.4643899603 & 0.1226615814 & 0.1517361862 & 3.8478500278 & 0.0116130644 & 0.3336864832 & 0.2012598078 \\
\hline & $a 0$ & 0.0000013223 & 0.0387077374 & 0 & 0.0003111686 & 0.1535906434 & 0.0000136045 & 0.0112262603 & 0.0063804478 \\
\hline \multirow{6}{*}{$y_{I I}$} & $a 5$ & 0 & 0 & 1 & 0 & 0 & 0 & 0 & 1 \\
\hline & a4 & 1 & 0 & 0.0434593880 & 0 & 0 & 1 & 0 & 0.1750310058 \\
\hline & $a 3$ & 0.1479431059 & 0 & 0.0020290995 & 1 & 1 & 1.9631340720 & 0 & 0.0060747686 \\
\hline & $a 2$ & 0.0027004884 & 1 & 0.0000348783 & 0.1658187045 & 0.1474516580 & 0.2991203847 & 1 & 0.0006895879 \\
\hline & $a 1$ & 0.0000060077 & 0.0138249033 & 0.0000008874 & 0.0023489638 & 0.0022809940 & 0.0042941403 & 0.0007605556 & 0.0000085470 \\
\hline & $a 0$ & 0.0000001080 & 0.0000060835 & 0 & 0.0000003977 & 0 & 0.0000000670 & 0.0000526818 & 0.0000000619 \\
\hline
\end{tabular}


Table 34 - Plant 3 - Sub-model 1 - Transfer Function Numerator Parameters.

\begin{tabular}{|c|c|c|c|c|c|c|c|c|c|}
\hline$P I$ & $N$ & $u_{l}$ & $u_{2}$ & $u_{3}$ & $u_{4}$ & $u_{5}$ & $u_{6}$ & $u_{7}$ & $d_{I}$ \\
\hline \multirow{6}{*}{$y_{l}$} & $b 5$ & 0 & 0 & 0 & 0 & 0 & 0 & 0 & 0 \\
\hline & $b 4$ & 0.0088265377 & 0 & 0 & 0.1148066535 & 0 & -0.0000617049 & 0.0056779129 & 0 \\
\hline & $b 3$ & 0.0032902506 & 0 & 0 & 0.0774254574 & 0.0078000437 & 0.0114822949 & -0.0012490954 & 0 \\
\hline & $b 2$ & 0.0003150114 & -0.0258105881 & -0.0757127689 & 0.0082045612 & -0.0019656391 & -0.0026171062 & -0.0031390142 & -0.0029029759 \\
\hline & $b 1$ & 0.0000041281 & 0.5849528973 & 0.0116802676 & 0.0000282916 & -0.0038822835 & -0.0048766530 & -0.0000017349 & -0.0000723765 \\
\hline & bo & 0.0000000510 & -0.0149272364 & 0.0061516246 & 0.0000006085 & 0.0000001888 & -0.0000100091 & -0.0000027660 & -0.0000069582 \\
\hline \multirow{6}{*}{$y_{2}$} & $b 5$ & -0.0039096482 & 0 & 0 & 0.0000258205 & 0 & 0 & 0 & -0.0038565107 \\
\hline & $b 4$ & 0.0707643044 & 0 & -0.1960958602 & 0.7428811725 & 0 & 0 & 0.0003487225 & 0.0642953837 \\
\hline & $b 3$ & 0.0578356360 & -0.0493317362 & -0.1388095299 & 0.9875932485 & 1.1629408914 & 0.5514690706 & 0.0578688278 & -0.0316719715 \\
\hline & $b 2$ & 0.0068412453 & 0.5632949179 & 0.1393578490 & 1.2819935417 & -0.3309061086 & -0.2555883639 & -0.0130848198 & -0.0149671831 \\
\hline & $b l$ & 0.0000518072 & 1.0262823462 & 0.8795715778 & 0.1125098406 & -0.3955333648 & -0.1413999845 & -0.0223051227 & -0.0004341016 \\
\hline & bo & 0.0000013232 & 0.0400236244 & 0.1017949219 & 0.0002946908 & -0.0306112196 & -0.0066498750 & -0.0021445081 & -0.0000348271 \\
\hline \multirow{6}{*}{$y_{3}$} & $b 5$ & 0 & 0 & 0 & 0 & 0 & 0.0010956671 & 0.0002767085 & -0.0079085427 \\
\hline & $b 4$ & -0.0009043812 & 0 & -0.2033617575 & 0 & 0.1731163471 & 0.2145907014 & 0.1398816110 & 0.1602123021 \\
\hline & $b 3$ & -0.1103957089 & 0 & 0.1982914405 & 0 & -0.0298955412 & -0.0345505893 & -0.0497696964 & -0.1070915423 \\
\hline & $b 2$ & 0.3100273231 & 0.5916043620 & 0.1365419591 & 0 & -0.0806847628 & -0.0977007066 & -0.0502784387 & -0.0264095833 \\
\hline & $b 1$ & 0.3099043910 & 0.6165535354 & 0.0081760591 & 16.3121853737 & -0.0069315319 & -0.0073931353 & -0.0026991857 & -0.0009044360 \\
\hline & bo & 0.0207475447 & -0.0671774358 & 0.0000559529 & 1.9215007790 & -0.0000037294 & -0.0000008061 & -0.0000020235 & -0.0000590189 \\
\hline \multirow{6}{*}{$y_{4}$} & $b 5$ & 0 & 0 & 0 & 0 & -0.0003545851 & 0 & 0.0002887180 & 0 \\
\hline & $b 4$ & -0.0195094886 & 0 & -0.2605066146 & 0 & 0.0801275690 & 0.0983953472 & 0.0697419224 & 0 \\
\hline & $b 3$ & 0.0909114681 & 0 & 0.0467562611 & 0 & -0.0034139430 & 0.0083311167 & -0.0230844811 & 0.0321276965 \\
\hline & b2 & 0.0070711099 & 0 & 0.1390273359 & 0 & -0.0564177330 & -0.0710786167 & -0.0330389274 & -0.0642091967 \\
\hline & $b 1$ & 0.0001939331 & 2.3949561661 & 0.0220481508 & 31.9946799923 & -0.0040492612 & -0.0077383904 & -0.0021999788 & -0.0017565333 \\
\hline & bo & 0.0000147083 & -0.3220977687 & 0.0008159501 & 4.6345899078 & -0.0000051504 & -0.0000016178 & -0.0000543340 & -0.0001414797 \\
\hline \multirow{6}{*}{$y_{5}$} & $b 5$ & 0 & 0 & 0 & 0.0015950460 & 0 & 0 & 0 & 0 \\
\hline & $b 4$ & 0 & 0 & 0 & 0.6325692292 & -0.0002152645 & 0 & 0.0232248731 & -0.0032027931 \\
\hline & $b 3$ & -1.4331843106 & 0 & 0 & 0.2048819219 & 0.0358501529 & 0 & 0.0202420286 & 0.0111445825 \\
\hline & $b 2$ & 2.0498547018 & 0.2388432977 & 0 & 0.0169193848 & 0.0032407326 & 0 & -0.0392489436 & -0.0228388709 \\
\hline & $b 1$ & 0.0000249920 & -0.0271222814 & -2.3662893349 & 0.0012400850 & -0.0333401267 & -0.0465852208 & 0.0000374170 & -0.0005267533 \\
\hline & bo & 0.0014582277 & -0.0011019907 & 2.3908854272 & 0.0000479255 & -0.0000261511 & -0.0003934891 & -0.0000650229 & -0.0000585280 \\
\hline \multirow{6}{*}{$y_{6}$} & $b 5$ & 0 & 0 & 0 & 0 & 0.0004255303 & 0 & 0 & -0.0035621339 \\
\hline & $b 4$ & -0.0001993029 & 0 & -0.0730081339 & 0 & 0.0947819198 & 0 & 0 & 0.6508748026 \\
\hline & $b 3$ & -0.0488922018 & 0.0362862725 & 0.0429753535 & 0 & -0.0181301911 & 0.7958644884 & 1.8474594330 & -0.3447143136 \\
\hline & $b 2$ & 0.1524119450 & 0.2554372844 & 0.0685545186 & 0 & -0.0380285671 & -0.4378828407 & -0.6884694848 & -0.0181009459 \\
\hline & $b l$ & 0.1609509211 & 0.0670647409 & 0.0058467833 & 4.0285151031 & -0.0021521860 & -0.1684534920 & -0.5956396808 & -0.0012913937 \\
\hline & bo & 0.0093404961 & -0.0079507790 & 0.0001296385 & 0.9388559704 & -0.0000027027 & -0.0006366489 & -0.0227983311 & -0.0000490580 \\
\hline \multirow{6}{*}{$y_{7}$} & $b 5$ & 0 & 0 & 0 & 0 & 0 & 0 & 0 & 0 \\
\hline & $b 4$ & 0 & 0.0003241525 & 0 & 0 & 0 & 0 & 0 & 0 \\
\hline & $b 3$ & -0.1306272230 & -0.0480606686 & -4.1243525373 & 0 & 148.1765787534 & 83.0834925148 & 0 & 0.2927647102 \\
\hline & $b 2$ & 0.1911461735 & 0.0836295155 & -6.1844755747 & 0 & 192.2670447375 & 160.3316323779 & 0.3376626862 & -0.0090080920 \\
\hline & $b l$ & 0.6326307601 & 0.0138171154 & -1.1851615513 & 4.8059581634 & -24.4777095916 & -26.4063505361 & 0.0046509577 & 0.0004088230 \\
\hline & bo & 0.0926010159 & -0.0045004903 & -0.0517847126 & 0.6320786479 & -7.6814565117 & -7.9399927693 & -0.0145975139 & -0.0000544584 \\
\hline \multirow{6}{*}{$y_{8}$} & $b 5$ & 0 & 0 & 0 & 0.0000919421 & 0 & -0.0017356215 & 0 & 0.0125468543 \\
\hline & $b 4$ & 0 & 0 & 0 & -0.0058194907 & 0 & 0.0322926547 & 0 & -0.0176851611 \\
\hline & $b 3$ & 0 & 0 & 0.0018431750 & 0.0014470922 & 0 & -0.0044007366 & -0.0169669526 & -0.0047684193 \\
\hline & $b 2$ & 0 & 0 & 0.0005927737 & -0.0000222052 & 0.0505149412 & -0.0502010419 & -0.0019167301 & -0.0001406101 \\
\hline & $b l$ & 0.3536987244 & 0.5087488668 & 0.0000004105 & 0.0000028158 & -0.0519237853 & -0.0003217669 & -0.0000345536 & -0.0000134945 \\
\hline & bo & 0.0182118185 & -0.1263767806 & 0.0000000174 & -0.0000000161 & -0.0001548623 & -0.0000032684 & -0.0000038000 & -0.0000002523 \\
\hline \multirow{6}{*}{$y_{9}$} & $b 5$ & 0 & 0 & 0 & 0 & 0 & 0 & 0 & 0 \\
\hline & $b 4$ & 0.0270166590 & 0 & -0.0017986188 & 0 & 0 & 0 & 0 & 0 \\
\hline & $b 3$ & 0.0051171588 & 0 & 0.0017198022 & 0 & 0 & 0.0442326090 & 0.0159780365 & 0 \\
\hline & b2 & 0.0002748816 & 0.0413796129 & 0.0005921534 & 0.0000459729 & 0.1412746290 & -0.0672689292 & -0.0287456332 & -0.0049872295 \\
\hline & $b l$ & 0.0000113360 & -0.0021155042 & 0.0000144183 & 0.0000001023 & -0.1283318760 & -0.0084394240 & -0.0031337366 & -0.0001281669 \\
\hline & bo & 0.0000004667 & -0.0003840880 & 0.0000000183 & 0.0000000066 & -0.0129213440 & -0.0000025141 & -0.0000375047 & -0.0000117683 \\
\hline \multirow{6}{*}{$y_{10}$} & $b 5$ & 0 & 0 & -0.0002329884 & 0 & 0.0001945052 & 0 & 0.0001500766 & 0 \\
\hline & $b 4$ & 0.0346272897 & 0.0000291861 & 0.0004924264 & 0 & 3.1342867044 & 0.0764542171 & 0.0314577474 & 0 \\
\hline & $b 3$ & 0.0048306266 & -0.0130930416 & 0.0000046642 & -0.0001709291 & -1.3961641608 & -0.0342246943 & -0.0048573904 & 0 \\
\hline & $b 2$ & 0.0001887476 & 0.0359678091 & 0.0000014958 & 0.0000917689 & -0.1070289033 & -0.0096250696 & -0.0066050925 & -0.0029198309 \\
\hline & $b 1$ & 0.0000162736 & -0.0050282540 & -0.0000000032 & 0.0000003677 & -0.0026111186 & -0.0001302558 & -0.0000046493 & -0.0000686361 \\
\hline & bo & 0.0000002659 & -0.0012887783 & 01 & 0.0000000414 & -0.0001881337 & -0.0000060997 & -0.0000027361 & -0.0000081407 \\
\hline \multirow{6}{*}{$y_{I I}$} & $b 5$ & 0 & 0 & 0.0000018737 & 0 & 0 & 0 & 0 & 0.0018521551 \\
\hline & $b 4$ & 0 & 0 & -0.0022431971 & -0.0006424568 & 0 & 0 & 0 & -0.0067241188 \\
\hline & $b 3$ & 0.1051089725 & -0.0024466439 & 0.0013955060 & 0.0003785498 & 0.0219737666 & 0.0105784662 & 0.0245907406 & -0.0006535329 \\
\hline & b2 & 0.1565109935 & 0.0221512126 & 0.0004965740 & 0.0000037643 & -0.0125871686 & -0.0327703927 & -0.0433200762 & -0.0000394042 \\
\hline & $b l$ & 0.0199789880 & -0.0076288055 & 0.0006027662 & 0.0000008628 & -0.0175910544 & -0.0000057333 & -0.0005842263 & -0.0000016700 \\
\hline & bo & 0.0000105473 & -0.0002975147 & 0.0000005740 & 0.0000000043 & -0.0000116030 & -0.0000046244 & -0.0000309957 & -0.0000000149 \\
\hline
\end{tabular}


Table 35 - Plant 3 - Sub-model 1 - Transfer Function Denominator Parameters.

\begin{tabular}{|c|c|c|c|c|c|c|c|c|c|}
\hline$P I$ & $D$ & $u_{l}$ & $u_{2}$ & $u_{3}$ & $u_{4}$ & $u_{5}$ & $u_{6}$ & $u_{7}$ & $d_{l}$ \\
\hline \multirow{6}{*}{$y_{l}$} & $a 5$ & 1 & 1 & 0 & 1 & 0 & 0 & 1 & 1 \\
\hline & a4 & 0.5420352999 & 8.0997348473 & 1 & 2.3869080177 & 1 & 1 & 1.4276071350 & 0.9009321133 \\
\hline & $a 3$ & 0.0838202251 & 19.3943925899 & 3.0050675717 & 1.2303588213 & 1.7316577218 & 1.7515205325 & 1.1733907369 & 1.1983509221 \\
\hline & $a_{2}$ & 0.0043612914 & 25.2111950271 & 7.4568623565 & 0.0950071648 & 1.2244885743 & 1.2245796060 & 0.0990416196 & 0.1346417397 \\
\hline & $a l$ & 0.0000587865 & 15.7308369225 & 2.2305534753 & 0.0003576191 & 0.0972009874 & 0.1019277635 & 0.0010388924 & 0.0057199256 \\
\hline & $a 0$ & 0.0000005338 & 1.3031046440 & 0.1472555752 & 0.0000068000 & 0.0000082843 & 0.0001673819 & 0.0000855731 & 0.0002286027 \\
\hline \multirow{6}{*}{$y_{2}$} & $a 5$ & 1 & 1 & 1 & 1 & 1 & 1 & 0 & 1 \\
\hline & a4 & 1.0451917605 & 3.2031584009 & 20.1809928713 & 11.4872832543 & 15.0081649321 & 6.1080777537 & 1 & 2.1473339271 \\
\hline & $a 3$ & 0.2863526382 & 7.3152749558 & 29.8328307695 & 15.1796611678 & 28.7770606974 & 11.7200341127 & 1.8132397804 & 1.4128656129 \\
\hline & $a 2$ & 0.0168520323 & 7.4044811660 & 42.1170592104 & 15.0295527146 & 22.5011722877 & 7.2475549629 & 1.5337793339 & 0.1576429838 \\
\hline & al & 0.0001565830 & 4.5760072507 & 15.8073351046 & 1.1703213263 & 4.0495433917 & 0.9666258010 & 0.3160632902 & 0.0066979271 \\
\hline & $a 0$ & 0.0000027823 & 0.4637748046 & 1.1585537176 & 0.0030872546 & 0.1769979908 & 0.0306874812 & 0.0158200344 & 0.0002642918 \\
\hline \multirow{6}{*}{$y_{3}$} & $a 5$ & 0 & 1 & 1 & 0 & 1 & 1 & 1 & 1 \\
\hline & $a 4$ & 1 & 2.3086378016 & 2.6197167746 & 1 & 1.9467407210 & 1.9249428705 & 1.8284486520 & 2.2613972464 \\
\hline & $a 3$ & 2.1511435309 & 4.8310667085 & 2.0764588783 & 5.8084314488 & 1.6519098957 & 1.6363781849 & 1.3820765884 & 1.2340026311 \\
\hline & $a 2$ & 2.6993655636 & 4.2951520880 & 0.4342313827 & 31.9441054075 & 0.4100443925 & 0.3913131619 & 0.2783000212 & 0.1443269922 \\
\hline & $a l$ & 0.7258880651 & 2.5138165763 & 0.0203402531 & 47.9974534683 & 0.0227740954 & 0.0197615693 & 0.0110084894 & 0.0061252832 \\
\hline & $a 0$ & 0.0344441703 & 0.3838913084 & 0.0001359016 & 4.6163321593 & 0.0000096091 & 0 & 0.0000091735 & 0.0002504049 \\
\hline \multirow{6}{*}{$y_{4}$} & $a 5$ & 0 & I & 1 & 0 & $I$ & 1 & 1 & 1 \\
\hline & $a 4$ & 1 & 5.3598101911 & 2.8591708683 & 1 & 1.5949844551 & 1.6933235365 & 1.5803362173 & 1.1033836709 \\
\hline & $a 3$ & 0.2251875118 & 12.4926620123 & 2.2417079420 & 6.0947788633 & 1.4110055096 & 1.5059902781 & 1.0926074734 & 1.9948612470 \\
\hline & $a 2$ & 0.0115399278 & 13.8988482383 & 0.5201517755 & 48.6768134884 & 0.2426325792 & 0.3074331450 & 0.1709470658 & 0.2901370838 \\
\hline & al & 0.0004741857 & 9.5351062667 & 0.0439748981 & 75.3745687858 & 0.0100503835 & 0.0158161869 & 0.0083757216 & 0.0113091511 \\
\hline & $a 0$ & 0.0000198659 & 0.9528042112 & 0.0012035139 & 6.7816941578 & 0.0000124724 & 0.0000015847 & 0.0001662860 & 0.0004545131 \\
\hline \multirow{6}{*}{$y_{5}$} & $a 5$ & 0 & 1 & 0 & 1 & 0 & 1 & 1 & 0 \\
\hline & $a 4$ & 1 & 1.8713547307 & 1 & 2.5629963412 & 1 & 1.6082723890 & 1.6469246769 & 1 \\
\hline & $a 3$ & 35.9861829997 & 2.4200232367 & 8.0115818562 & 0.4110356330 & 1.6494422176 & 2.1445802043 & 1.5824570143 & 1.0549331742 \\
\hline & $a 2$ & 2.8727792217 & 1.2142883512 & 22.9247121578 & 0.0336515259 & 1.1331198937 & 1.3306434961 & 0.1226738205 & 0.1036841544 \\
\hline & $a l$ & 0.0270069649 & 0.1171852194 & 38.8299892996 & 0.0021348753 & 0.0831071985 & 0.1058364062 & 0.0026080906 & 0.0046809970 \\
\hline & $a 0$ & 0.0020173140 & 0.0026099516 & 3.2367233062 & 0.0000625864 & 0.0000542774 & 0.0007724304 & 0.0001979098 & 0.0001855032 \\
\hline \multirow{6}{*}{$y_{6}$} & $a 5$ & 0 & 1 & 1 & 0 & 1 & 1 & 1 & 1 \\
\hline & a4 & 1 & 1.9408888239 & 2.5475515435 & 1 & 2.0667435717 & 7.7306510551 & 26.6684770842 & 12.6398839832 \\
\hline & $a 3$ & 2.2054242323 & 3.2699327462 & 2.6900699928 & 4.7344257284 & 1.7776267628 & 14.2375588546 & 50.1852163500 & 5.6815009258 \\
\hline & $a 2$ & 2.9235643324 & 2.4953574234 & 0.7930457057 & 16.8786855124 & 0.4307778915 & 8.9368405390 & 38.6659041382 & 0.2862004856 \\
\hline & al & 0.8636984246 & 1.0248401303 & 0.0542064478 & 28.2262187579 & 0.0185694369 & 1.2133687887 & 7.8153551784 & 0.0197788641 \\
\hline & $a 0$ & 0.0387529528 & 0.1335654221 & 0.0010990883 & 5.1788204688 & 0.0000232408 & 0.0040521906 & 0.2445554744 & 0.0005477836 \\
\hline \multirow{6}{*}{$y_{7}$} & $a 5$ & $I$ & 0 & 1 & 0 & 1 & 1 & 0 & 1 \\
\hline & $a 4$ & 7.3917026348 & 1 & 6.8246384925 & 1 & 1805.9596748276 & 948.3495199444 & 1 & 2.0734325016 \\
\hline & $a 3$ & 16.9987542031 & 1.7392956975 & 19.3684508878 & 6.2878992224 & 4987.4841820789 & 3052.2536534611 & 4.7122255119 & 6.7594093878 \\
\hline & $a 2$ & 23.8969649199 & 1.6479796097 & 20.0938014038 & 32.9526072471 & 6129.3282656364 & 4194.3895596366 & 9.1929229152 & 1.2516181780 \\
\hline & $a l$ & 9.1460099059 & 0.6404981404 & 4.3658855502 & 50.3521540302 & 2020.4876010679 & 1525.4928131253 & 4.4087552783 & 0.0506197671 \\
\hline & $a 0$ & 0.6593463905 & 0.0561753656 & 0.2317566708 & 4.4008996768 & 136.8468743808 & 107.0143049482 & 0.3592429484 & 0.0023315967 \\
\hline \multirow{6}{*}{$y_{8}$} & $a 5$ & 0 & 1 & 0 & $I$ & 0 & 1 & 1 & 1 \\
\hline & a4 & 0 & 4.8703871565 & 1 & 1.9795342521 & 0 & 1.7283884398 & 0.7293039537 & 0.3090645789 \\
\hline & $a 3$ & 1 & 9.2235405981 & 0.3178278661 & 0.0925333203 & 1 & 1.2818755606 & 0.1362030777 & 0.0273547056 \\
\hline & $a 2$ & 3.9080105692 & 10.8683050812 & 0.0231679662 & 0.0048792004 & 1.3959563496 & 0.1097421928 & 0.0077285200 & 0.0012071585 \\
\hline & al & 0.5872469870 & 5.8224828287 & 0.0000137355 & 0.0001841375 & 0.1413582106 & 0.0008050927 & 0.0002734883 & 0.0000684287 \\
\hline & $a 0$ & 0.0190596374 & 0.5087515264 & 0.0000009984 & 0.0000001778 & 0.0003862549 & 0.0000044551 & 0.0000127238 & 0.0000009220 \\
\hline \multirow{6}{*}{$y_{9}$} & $a 5$ & 1 & 1 & 1 & 0 & 0 & 1 & 1 & 0 \\
\hline & $a 4$ & 0.3054785764 & 2.3359906413 & 1.2335275727 & 1 & 0 & 3.0239267732 & 2.2490124256 & 0 \\
\hline & $a 3$ & 0.0290146519 & 3.4431425918 & 2.3802456141 & 0.4877599682 & $I$ & 7.1158006163 & 5.3164043879 & 1 \\
\hline & $a 2$ & 0.0012740843 & 2.5009564719 & 0.1986109627 & 0.0319638579 & 16.9856424413 & 1.6514622181 & 1.0697407423 & 0.1004546643 \\
\hline & al & 0.0000588735 & 0.4486206167 & 0.0037346661 & 0.0000167656 & 3.4826251058 & 0.0618429212 & 0.0476154220 & 0.0046348653 \\
\hline & $a 0$ & 0.0000013248 & 0.0152850975 & 0.0000000165 & 0.0000010975 & 0.1195352183 & 0.0000220420 & 0.0004628941 & 0.0001398355 \\
\hline \multirow{6}{*}{$y_{I 0}$} & $a 5$ & 1 & 0 & 1 & 0 & 1 & 1 & 1 & 1 \\
\hline & $a 4$ & 0.2745828135 & 1 & 0.1118098203 & 1 & 151.1456953548 & 3.7849948084 & 2.1979487995 & 1.4822657760 \\
\hline & $a 3$ & 0.0221439790 & 1.6428972026 & 0.0045847365 & 0.2075031157 & 29.8362997861 & 1.2384676028 & 1.2308065859 & 0.6134784506 \\
\hline & $a 2$ & 0.0011753068 & 1.6807687745 & 0.0002698688 & 0.0334946658 & 1.4454349779 & 0.0975105773 & 0.0982558705 & 0.0673643158 \\
\hline & $a l$ & 0.0000625557 & 0.4229118048 & 0.0000001560 & 0.0000824670 & 0.0528394850 & 0.0016340659 & 0.0005112244 & 0.0031395107 \\
\hline & $a 0$ & 0.0000008729 & 0.0247006258 & 0.0000000091 & 0.0000131328 & 0.0019356836 & 0.0000525676 & 0.0000404456 & 0.0001139918 \\
\hline \multirow{6}{*}{$y_{H}$} & $a 5$ & 1 & 0 & 1 & 1 & 0 & 1 & 1 & 1 \\
\hline & a4 & 3.0394706468 & 1 & 2.0805845617 & 1.3188140016 & $I$ & 1.5831727509 & 3.1574527976 & 0.2425215160 \\
\hline & $a 3$ & 5.9801864243 & 1.2716332870 & 2.6792576219 & 0.2627269411 & 3.0847056885 & 2.7941272468 & 5.8715913401 & 0.0189393657 \\
\hline & $a 2$ & 1.4946073937 & 1.3222869137 & 1.6037916380 & 0.0031240341 & 2.1548732063 & 0.3706318470 & 0.9240439721 & 0.0011378699 \\
\hline & al & 0.0996855891 & 0.2747634656 & 0.1994905451 & 0.0005475502 & 0.2590855297 & 0.0004023455 & 0.0156097946 & 0.0000334105 \\
\hline & $a 0$ & 0.0000887906 & 0.0080078656 & 0.0001283311 & 0.0000000980 & 0.0001393897 & 0.0000533396 & 0.0005962020 & 0.0000002805 \\
\hline
\end{tabular}


Table 36 - Plant 3 - Sub-model 2 - Transfer Function Numerator Parameters.

\begin{tabular}{|c|c|c|c|c|c|c|c|c|c|}
\hline$P I$ & $N$ & $u_{l}$ & $u_{2}$ & $u_{3}$ & $u_{4}$ & $u_{5}$ & $u_{6}$ & $u_{7}$ & $d_{I}$ \\
\hline \multirow{6}{*}{$y_{l}$} & $b 5$ & 0 & 0 & 0 & 0 & 0 & 0 & 0 & 0 \\
\hline & $b 4$ & 0 & -0.0114852360 & 0 & 0 & 0 & 0 & 0 & 0 \\
\hline & $b 3$ & -0.0023079261 & 0.0164335978 & -0.0200895799 & 0 & -0.0004055095 & 0 & 0 & 0.0181282697 \\
\hline & $b 2$ & 0.0034330665 & -0.0007400408 & 0.0032745330 & -0.1003064131 & 0.0000055145 & -0.0001909173 & -0.0008010328 & -0.0072229269 \\
\hline & $b 1$ & -0.0000022186 & 0.0000079968 & 0.0000056640 & -0.0121859332 & -0.0000009959 & -0.0000010139 & -0.0000814506 & -0.0005997186 \\
\hline & bo & 0.0000004643 & -0.0000006004 & 0.0000003346 & 0.0000031483 & 0.0000000049 & -0.0000001336 & 0.0000000099 & -0.0000415031 \\
\hline \multirow{6}{*}{$y_{2}$} & $b 5$ & 0 & 0 & 0 & 0 & 0.0001807522 & 0 & 0 & 0 \\
\hline & $b 4$ & 0.0118910160 & 0 & 0 & 0 & 0.1033978507 & 0 & 0.0003384502 & 0 \\
\hline & $b 3$ & 0.0367370167 & 0 & 0 & 0 & -0.0548384868 & 0.1505536789 & 0.0621832549 & 0.6615889000 \\
\hline & $b 2$ & 0.0041981086 & 0 & -0.0578403402 & 0 & -0.0112477858 & -0.0724879498 & -0.0367419712 & -0.2421566706 \\
\hline & $b l$ & 0.0000507331 & 1.9043147866 & 0.1133910438 & -268.0677337434 & -0.0000810825 & -0.0153807854 & -0.0072544176 & -0.0635182366 \\
\hline & bo & 0.0000003630 & 0.0932461342 & 0.0388239502 & -182.6822578418 & -0.0000001757 & -0.0000001261 & -0.0000001948 & -0.0011890148 \\
\hline \multirow{6}{*}{$y_{3}$} & $b 5$ & 0 & -0.0013114288 & -0.0012280469 & 0 & 0 & 0 & 0 & 0 \\
\hline & $b 4$ & 0 & -0.0407344766 & -0.2003386419 & 0 & 0.2348672648 & 0.2815438819 & 0.1773960062 & 0 \\
\hline & $b 3$ & 0 & 0.1027854980 & 0.2070622905 & 0 & -0.1417372042 & -0.1366884928 & -0.1028141678 & 0 \\
\hline & $b 2$ & 0.5890488437 & 0.2877992619 & 0.0634013217 & 0 & -0.0474004927 & -0.0787310859 & -0.0445187150 & 0.4362402392 \\
\hline & $b 1$ & 0.1027456076 & -0.0092834763 & 0.0003721147 & -4641.7907678691 & -0.0030167615 & -0.0052771691 & -0.0028509980 & -0.5250847724 \\
\hline & bo & 0.0000590585 & -0.0047585806 & 0.0001144129 & -2321.4891731725 & -0.0000370251 & -0.0000319270 & -0.0000225462 & -0.0567903099 \\
\hline \multirow{6}{*}{$y_{4}$} & $b 5$ & 0 & 0 & 0 & 0 & 0 & 0 & 0 & 0 \\
\hline & $b 4$ & 0 & 0 & 0 & -0.9481559382 & 0.1527737729 & 0 & 0.1280244577 & 0 \\
\hline & $b 3$ & 0.1159177449 & 0 & 0 & -0.2849407076 & -0.1020343762 & 0.1648350547 & -0.0846065740 & 0 \\
\hline & b2 & 0.0022243464 & 0.3355772504 & 0.1051544571 & -0.0253977703 & -0.0199126161 & -0.0738167036 & -0.0157495007 & 0.4383647567 \\
\hline & $b 1$ & 0.0000564409 & -0.0389667947 & 0.0099810141 & -0.0005948706 & -0.0000000833 & -0.0582736998 & -0.0000006280 & -0.4579870372 \\
\hline & bo & 0.0000008737 & -0.0043783719 & 0.0000692159 & -0.0000428554 & -0.0000001511 & -0.0039496135 & -0.0000002129 & -0.0692368603 \\
\hline \multirow{6}{*}{$y_{5}$} & $b 5$ & 0 & 0 & 0 & 0 & 0 & 0 & 0.0005210000 & 0 \\
\hline & $b 4$ & 0 & -0.0994365257 & 0.0004990659 & -0.0027974626 & 0 & 0 & 0.0495148577 & 0 \\
\hline & $b 3$ & 0 & 0.1868729469 & -0.2500369572 & -0.6397489267 & 0 & 0.0710277604 & -0.0480584462 & 0.0497685957 \\
\hline & $b 2$ & 0.0881384448 & -0.0221563544 & 0.1808098062 & -0.1850037621 & 0.0685838279 & -0.0642340841 & -0.0006756610 & -0.0480902252 \\
\hline & $b 1$ & -0.0000810388 & -0.0015063329 & 0.0010486460 & -0.0103702393 & -0.0587518169 & -0.0021622788 & -0.0003030340 & -0.0024717538 \\
\hline & bo & 0.0000214652 & -0.0001630454 & 0.0001727514 & -0.0000000442 & -0.0008656014 & -0.0000069189 & -0.0000062280 & -0.0000231190 \\
\hline \multirow{6}{*}{$y_{6}$} & $b 5$ & 0 & 0 & 0 & 0 & 0 & 0 & 0 & 0.0004224611 \\
\hline & $b 4$ & 0 & 0 & 0 & 0 & 0.1177128734 & 0 & 0.0900648011 & 0.0995328700 \\
\hline & $b 3$ & 0.3222398244 & 0 & 0 & -0.3058024990 & -0.0310029078 & 0 & -0.0413039318 & -0.0336578910 \\
\hline & $b 2$ & 0.0510489911 & 0.1865504605 & -0.1562780715 & -0.1499519708 & -0.0470255178 & 0.1633647422 & -0.0257349915 & -0.0390018704 \\
\hline & $b l$ & 0.0000834190 & -0.0107780654 & 0.1926911970 & -0.0041383703 & -0.0028027845 & -0.1104021718 & -0.0018712208 & -0.0028855661 \\
\hline & bo & 0.0000027520 & -0.0015547693 & 0.0495014336 & -0.0000127414 & 0.0000001237 & -0.0129850840 & -0.0000016240 & -0.0000659441 \\
\hline \multirow{6}{*}{$y_{7}$} & $b 5$ & 0 & 0 & 0 & 0 & 0 & 0 & 0 & 0 \\
\hline & $b 4$ & 0 & -0.0233585654 & 0 & 0 & 0 & 0 & 0 & 0.0787489714 \\
\hline & $b 3$ & 0 & 0.0536869346 & 0 & 0 & 1.9852945801 & 1.3825742162 & 0 & 0.0902797164 \\
\hline & $b 2$ & 0.0553082773 & -0.0044984571 & -3.4375137306 & 0 & -0.1738376749 & -0.1717429734 & 5.5515058049 & -0.0177177061 \\
\hline & $b l$ & 0.0189839789 & -0.0012705472 & -3.0720065887 & -6.0688182519 & -0.0757639938 & -0.0678536843 & -0.0526347885 & 0.0000962323 \\
\hline & bo & 0.0000103471 & -0.0000567210 & -0.1973736792 & -0.7376776812 & -0.0002263665 & -0.0002855750 & -0.1917480314 & -0.0000180310 \\
\hline \multirow{6}{*}{$y_{8}$} & $b 5$ & -0.0031915945 & 0.0001144851 & 0 & 0 & 0.0000678504 & 0 & -0.0000048991 & 0 \\
\hline & $b 4$ & 0.0178052469 & -0.0029515215 & 0 & 0 & 0.0033807883 & 0 & 0.0034909828 & 0 \\
\hline & $b 3$ & 0.0096293268 & 0.0089411649 & 0 & 0 & -0.0062837031 & 0.0068151145 & -0.0052999489 & 0 \\
\hline & $b 2$ & 0.0005519233 & -0.0032831042 & 0 & -0.0001011497 & -0.0001790236 & -0.0101345745 & -0.0002013563 & -0.0101139640 \\
\hline & $b l$ & 0.0000018920 & -0.0000024212 & 0.0006209544 & -0.0000471890 & -0.0000085668 & 0.0000190508 & -0.0000097876 & 0.0000027954 \\
\hline & bo & 0.0000001298 & -0.0000105062 & 0.0000004228 & -0.0000002420 & -0.0000002434 & -0.0000194770 & -0.0000003732 & -0.0000137105 \\
\hline \multirow{6}{*}{$y_{9}$} & $b 5$ & 0 & -0.0019066345 & 0 & 0 & 0 & 0 & 0 & 0 \\
\hline & $b 4$ & 0 & 0.0160506820 & 0 & 0 & 0.0096258192 & 0 & 0.0059970572 & 0 \\
\hline & $b 3$ & 0.4256979415 & -0.0002309334 & 0 & -0.0011503691 & -0.0149514719 & 0 & -0.0114628089 & 0 \\
\hline & b2 & 0.0418674900 & -0.0004550253 & 0.5745911097 & -0.0001716955 & -0.0017204902 & -0.0291000722 & -0.0009102005 & -0.1531010564 \\
\hline & $b l$ & 0.0001544442 & -0.0000041360 & 0.1003061724 & -0.0000014942 & -0.0000471916 & -0.0019376101 & -0.0000154180 & -0.0193701613 \\
\hline & bo & 0.0000059923 & -0.0000030648 & 0.0000167308 & -0.0000000084 & -0.0000000182 & -0.0000013681 & -0.0000000811 & -0.0002240421 \\
\hline \multirow{6}{*}{$y_{10}$} & $b 5$ & 0 & 0 & 0 & 0 & 0 & 0 & 0 & 0 \\
\hline & $b 4$ & 0.0009385476 & 0 & 0 & 0 & 0 & 0 & 0 & 0 \\
\hline & $b 3$ & 0.0353316364 & 0 & 0 & -0.0016918754 & -0.0044324562 & 4.4121816527 & 0 & 0.2290544383 \\
\hline & $b 2$ & 0.0020826456 & 0.0096245123 & 0 & -0.0001836556 & -0.0007154779 & -2.2816782801 & 0.5502544024 & -0.1121062886 \\
\hline & $b 1$ & 0.0000151242 & -0.0046567497 & 0.0003037978 & -0.0000095520 & -0.0000284203 & -0.1539641039 & -0.2700462580 & -0.0069326925 \\
\hline & bo & 0.0000001925 & -0.0000318107 & -0.0000008120 & -0.0000000171 & -0.0000000439 & -0.0000474691 & -0.0002450115 & -0.0000197075 \\
\hline \multirow{6}{*}{$y_{H I}$} & $b 5$ & 0 & 0 & 0 & 0 & 0 & 0 & 0 & 0 \\
\hline & $b 4$ & 0.0907764945 & -0.0030141848 & 0 & 0 & 0 & 0 & 0 & 0 \\
\hline & $b 3$ & 0.0104081107 & 0.0315345810 & 0.0005351950 & 0.0048233699 & 0 & 0 & 0 & 0 \\
\hline & b2 & 0.0002632177 & -0.0179642877 & 0.0007609547 & -0.0025666591 & 0 & 0 & 0 & 0 \\
\hline & $b 1$ & 0.0000183248 & -0.0003301155 & 0.0000041637 & -0.0000247112 & -0.0502191488 & -0.0654406655 & -0.0387873745 & -0.1513733548 \\
\hline & bo & 0.0000001599 & -0.0000592867 & 0.0000000859 & -0.0000000645 & -0.0004321930 & -0.0023742147 & -0.0001834978 & -0.0025601818 \\
\hline
\end{tabular}


Table 37 - Plant 3 - Sub-model 2 - Transfer Function Denominator Parameters.

\begin{tabular}{|c|c|c|c|c|c|c|c|c|c|}
\hline$P I$ & $D$ & $u_{1}$ & $u_{2}$ & $u_{3}$ & $u_{4}$ & $u_{5}$ & $u_{6}$ & $u_{7}$ & $d_{l}$ \\
\hline \multirow{6}{*}{$y_{t}$} & $a 5$ & 0 & 1 & 0 & 0 & 1 & 1 & 0 & 0 \\
\hline & a4 & 0 & 1.7259684747 & 1 & 0 & 0.3279144091 & 0.4688894218 & 0 & 1 \\
\hline & $a 3$ & 1 & 1.1260394628 & 1.4849268612 & 1 & 0.0186549268 & 0.1283969563 & 1 & 8.6454335141 \\
\hline & $a_{2}$ & 0.0624803316 & 0.0531176183 & 0.1485259632 & 2.0340335389 & 0.0008712436 & 0.0108422404 & 0.1124333551 & 1.1610790672 \\
\hline & $a l$ & 0.0000864841 & 0.0017547225 & 0.0000747711 & 0.1661605821 & 0.0000416539 & 0.0000870359 & 0.0046822606 & 0.0822447156 \\
\hline & $a 0$ & 0.0000054025 & 0.0000201649 & 0.0000067989 & 0.0000031125 & 0.0000000539 & 0.0000071293 & 0.0000025833 & 0.0025990330 \\
\hline \multirow{6}{*}{$y_{2}$} & $a 5$ & 0 & 1 & 0 & 0 & 1 & 0 & 0 & 0 \\
\hline & a4 & 1 & 7.5728156988 & 1 & 0 & 3.7112049665 & 1 & 1 & 1 \\
\hline & $a 3$ & 0.2513917507 & 19.5850466537 & 4.8796669756 & 1 & 1.7896117013 & 4.0708320367 & 2.9659467266 & 19.0793252347 \\
\hline & $a 2$ & 0.0166660767 & 23.3288115612 & 8.0944369828 & 1698.9120211027 & 0.1311250472 & 1.9892932469 & 1.4549352186 & 10.5895524291 \\
\hline & $a l$ & 0.0001780432 & 16.7660469181 & 5.9488759717 & 7144.0268613974 & 0.0009054390 & 0.1357568738 & 0.0996467773 & 0.9188214656 \\
\hline & $a 0$ & 0.0000014830 & 1.7222133878 & 0.7533237887 & 3215.5911026662 & 0.0000020205 & 0.0000024215 & 0.0000076016 & 0.0144357970 \\
\hline \multirow{6}{*}{$y_{3}$} & $a 5$ & 0 & 1 & 1 & 0 & $I$ & 1 & 1 & 0 \\
\hline & $a 4$ & 1 & 1.6833249419 & 2.0714062343 & 0 & 2.2492446529 & 2.2534504718 & 2.1719249042 & $I$ \\
\hline & $a 3$ & 2.6133735853 & 2.3054162122 & 1.2315876929 & 1 & 1.2419800792 & 1.4459528846 & 1.3093402459 & 4.3111380638 \\
\hline & $a 2$ & 1.2597102590 & 1.3919552118 & 0.1407800628 & 3405.3155088275 & 0.1900901710 & 0.2649167355 & 0.2214249292 & 7.8080130436 \\
\hline & $a l$ & 0.1099838152 & 0.3576578743 & 0.0022065482 & 15389.6284493676 & 0.0091846794 & 0.0126633402 & 0.0104781508 & 2.8544726645 \\
\hline & $a 0$ & 0 & 0.0224327837 & 0.0002459237 & 5031.2740133113 & 0.0001042294 & 0.0000734378 & 0.0000787397 & 0.1769685932 \\
\hline \multirow{6}{*}{$y_{4}$} & $a 5$ & 0 & 1 & 1 & 1 & 1 & 0 & 1 & 0 \\
\hline & $a 4$ & 1 & 2.1308549906 & 1.5539747622 & 2.0192431458 & 2.1282038618 & 1 & 2.1506927219 & 1 \\
\hline & $a 3$ & 0.1200853459 & 2.6193615051 & 1.3892146228 & 0.4820623478 & 0.6612317669 & 2.1401587105 & 0.6524308528 & 5.3110624680 \\
\hline & $a 2$ & 0.0024347497 & 1.6837082748 & 0.2611270230 & 0.0337355304 & 0.0406821123 & 1.1973227011 & 0.0396112363 & 10.3268359313 \\
\hline & $a l$ & 0.0000583918 & 0.2625732519 & 0.0132765866 & 0.0009747076 & 0.0000054761 & 0.1714696977 & 0.0000120451 & 2.7869795133 \\
\hline & $a 0$ & 0.0000007392 & 0.0101822982 & 0.0000857916 & 0.0000535654 & 0.0000003014 & 0.0066737581 & 0.0000005139 & 0.1571999631 \\
\hline \multirow{6}{*}{$y_{5}$} & $a 5$ & 0 & 1 & 0 & 0 & 0 & 0 & 1 & 0 \\
\hline & $a 4$ & 1 & 1.7193729101 & 1 & 1 & 0 & 1 & 1.7708805305 & 1 \\
\hline & $a 3$ & 1.1808955921 & 1.0856363618 & 2.7292406209 & 2.2364681621 & 1 & 1.6379165814 & 0.1637261397 & 1.6970780825 \\
\hline & $a 2$ & 0.0725317278 & 0.1403677466 & 0.2268627719 & 0.3127798050 & 1.7379775428 & 0.1643908112 & 0.0136220648 & 0.1974406352 \\
\hline & $a l$ & 0.0002708010 & 0.0090832007 & 0.0040335893 & 0.0114904886 & 0.1464856031 & 0.0037835406 & 0.0009922651 & 0.0063898460 \\
\hline & $a 0$ & 0.0000165779 & 0.0003042935 & 0.0001955784 & 0.0000001968 & 0.0017413420 & 0.0000114768 & 0.0000155046 & 0.0000522597 \\
\hline \multirow{6}{*}{$y_{6}$} & $a 5$ & 1 & 1 & 0 & 0 & 1 & 0 & 1 & 1 \\
\hline & $a 4$ & 3.9216268673 & 1.8481040582 & 1 & 1 & 2.6031563748 & 0 & 2.5023336579 & 2.4872027412 \\
\hline & $a 3$ & 1.5966060996 & 2.7596054786 & 3.0261358025 & 2.2512197064 & 2.1738927610 & 1 & 1.7550131331 & 2.0885680008 \\
\hline & $a_{2}$ & 0.1580762893 & 1.8704570330 & 6.2143318557 & 0.8384293208 & 0.5160396382 & 2.6415495300 & 0.3650426259 & 0.4942870607 \\
\hline & $a l$ & 0.0001645189 & 0.4939342959 & 3.5455696662 & 0.0223064373 & 0.0226904361 & 1.2255865369 & 0.0192397863 & 0.0289756406 \\
\hline & $a 0$ & 0.0000145525 & 0.0240110503 & 0.4153945616 & 0.0000682973 & 0.0000006196 & 0.0861439240 & 0.0000166336 & 0.0005846857 \\
\hline \multirow{6}{*}{$y_{7}$} & $a 5$ & 0 & 1 & 0 & 0 & 1 & 1 & 0 & 1 \\
\hline & a4 & 1 & 1.3488935314 & 1 & 1 & 17.3122511519 & 11.9976759084 & 1 & 1.9678649967 \\
\hline & $a 3$ & 1.2997763883 & 1.1733262941 & 5.1916183331 & 5.9068121903 & 41.8751444681 & 26.6235248465 & 49.1040920453 & 2.8573263468 \\
\hline & $a 2$ & 0.8915096884 & 0.3006765964 & 13.0625851308 & 40.9277553695 & 17.4890422688 & 11.5416867951 & 124.1685889750 & 0.4330159321 \\
\hline & $a l$ & 0.0902840295 & 0.0235156591 & 9.6365956223 & 64.7360749552 & 1.2180997818 & 0.8508099286 & 56.2837393233 & 0.0028619843 \\
\hline & $a 0$ & 0 & 0.0006524709 & 0.8875345902 & 5.0876736612 & 0.0036442294 & 0.0034601223 & 4.0483156997 & 0.0004282429 \\
\hline \multirow{6}{*}{$y_{8}$} & $a 5$ & 1 & 1 & 0 & 0 & 1 & 0 & 1 & 1 \\
\hline & $a 4$ & 0.5878079185 & 1.1854260972 & 0 & 1 & 1.1996204165 & 1 & 1.3065952789 & 2.2661203315 \\
\hline & $a 3$ & 0.0704426256 & 0.8319660677 & 1 & 1.2837134834 & 0.1424915878 & 1.5058758877 & 0.1710408392 & 2.0483910523 \\
\hline & $a 2$ & 0.0024833276 & 0.0823302142 & 1.5646100368 & 0.5337851950 & 0.0043799176 & 0.1339736421 & 0.0065749799 & 0.1726748542 \\
\hline & $a l$ & 0.0000153561 & 0.0032674172 & 0.1492619064 & 0.0309051963 & 0.0001912812 & 0.0028599411 & 0.0003072318 & 0.0027759150 \\
\hline & $a 0$ & 0.0000005146 & 0.0002249889 & 0.0000811123 & 0.0001526724 & 0.0000037326 & 0.0002508342 & 0.0000076023 & 0.0002299913 \\
\hline \multirow{6}{*}{$y_{9}$} & $a 5$ & 1 & 1 & 0 & 1 & 0 & 0 & 0 & $I$ \\
\hline & $a 4$ & 6.9678913555 & 0.6308441336 & 1 & 5.4247181826 & 1 & 1 & 1 & 5.7274766296 \\
\hline & $a 3$ & 1.1110514369 & 0.1892057190 & 429.7969223364 & 1.3000930052 & 0.1891926947 & 1.4827973571 & 0.1530954394 & 10.2868093798 \\
\hline & $a_{2}$ & 0.0449668314 & 0.0132182148 & 232.2954426682 & 0.0667972895 & 0.0108780605 & 0.2129262449 & 0.0066323423 & 2.1245201115 \\
\hline & $a l$ & 0.0002187157 & 0.0010679454 & 11.4112771550 & 0.0004533462 & 0.0001853873 & 0.0059830272 & 0.0000865736 & 0.0966926808 \\
\hline & $a 0$ & 0.0000062137 & 0.0000478641 & 0.0024048135 & 0.0000008222 & 0.0000003511 & 0.0000060309 & 0.0000006513 & 0.0008979680 \\
\hline \multirow{6}{*}{$y_{10}$} & $a 5$ & 0 & 0 & 0 & 1 & 1 & 0 & 0 & 0 \\
\hline & a4 & 1 & 1 & 0 & 10.1405496473 & 0.7117908588 & 1 & 0 & I \\
\hline & $a 3$ & 0.1553255978 & 0.7841784974 & 0 & 2.0525553745 & 0.1549288932 & 217.2563658822 & 1 & 15.1369873778 \\
\hline & $a 2$ & 0.0060111252 & 0.8766096176 & 1 & 0.1527780926 & 0.0122859480 & 38.1725859208 & 43.8167285190 & 2.4489831736 \\
\hline & $a l$ & 0.0000428767 & 0.1025802201 & 0.0647889578 & 0.0052089611 & 0.0003157011 & 1.3085218261 & 4.1749030819 & 0.0822613150 \\
\hline & $a 0$ & 0.0000005956 & 0.0006514557 & 0.0000127418 & 0.0000005845 & 0.0000004819 & 0.0004162519 & 02 & 0.0002399978 \\
\hline \multirow{6}{*}{$y_{I I}$} & $a 5$ & 1 & 1 & 0 & 0 & 0 & 0 & 0 & 0 \\
\hline & a4 & 0.2373350513 & 1.0985346141 & 0 & 1 & $I$ & 1 & 1 & $I$ \\
\hline & $a 3$ & 0.0164699107 & 1.1454248512 & 1 & 5.3887026754 & 1.6002133768 & 1.7890719964 & 1.5671613641 & 4.2944189667 \\
\hline & $a 2$ & 0.0005203813 & 0.2211082973 & 0.0885136946 & 0.7792755619 & 2.3833322273 & 2.4826808752 & 2.4017021532 & 7.2901869601 \\
\hline & al & 0.0000259881 & 0.0078820348 & 0.0007153449 & 0.0077667983 & 0.2868002099 & 0.3651672206 & 0.2811487098 & 0.9442051583 \\
\hline & $a 0$ & 0.0000001783 & 0.0006046363 & 0.0000023497 & 0.0000156242 & 0.0021904437 & 0.0097407334 & 0.0011857124 & 0.0133891270 \\
\hline
\end{tabular}


Table 38 - Plant 3 - Sub-model 3 - Transfer Function Numerator Parameters.

\begin{tabular}{|c|c|c|c|c|c|c|c|c|c|}
\hline$P I$ & $N$ & $u_{I}$ & $u_{2}$ & $u_{3}$ & $u_{4}$ & $u_{5}$ & $u_{6}$ & $u_{7}$ & $d_{l}$ \\
\hline \multirow{6}{*}{$y_{l}$} & $b 5$ & 0 & 0 & -0.0007834414 & 0 & 0 & 0 & 0 & 0 \\
\hline & $b 4$ & -0.0338356492 & 0 & -0.0315665952 & 0 & -0.0000021439 & -0.0000192419 & 0 & 0.0045153607 \\
\hline & $b 3$ & 0.0301776976 & 0 & -0.0053121674 & 0 & 0.0040476663 & 0.0032452578 & 0.0010091304 & -0.0016282240 \\
\hline & $b_{2}$ & 0.0140949862 & 0 & 0.0006274727 & 0 & -0.0022995834 & -0.0013931431 & -0.0007928835 & -0.0009795173 \\
\hline & $b 1$ & 0.0002560498 & 0.1059939085 & -0.0000548763 & 2.1151558888 & -0.0006188477 & -0.0005838876 & -0.0008388003 & -0.0000011262 \\
\hline & bo & 0.0000008188 & -0.0004768229 & 0.0000103432 & 0.3311729829 & -0.0000224284 & -0.0000080936 & -0.0000005116 & -0.0000005073 \\
\hline \multirow{6}{*}{$y_{2}$} & $b 5$ & -0.0184846410 & 0 & -0.0002944467 & 0 & 0 & 0 & 0 & 0 \\
\hline & b4 & 0.0810384065 & 0 & -0.0219186891 & 0 & 0.0009506876 & 0.0006431024 & 0 & 0 \\
\hline & $b 3$ & 0.0088632961 & -0.0091839383 & 0.0220363425 & 0.0004264313 & 0.1142628429 & 0.0904472615 & 0.0466980902 & 74.8080846124 \\
\hline & $b 2$ & 0.0008055759 & 0.0628592683 & 0.0210985004 & 0.0642341737 & -0.0347750970 & -0.0214851714 & -0.0136025204 & -11.8632378735 \\
\hline & $b l$ & 0.0000014403 & 0.1265192953 & 0.0006871098 & 0.0220243148 & -0.0354048641 & -0.0283541830 & -0.0180585215 & -27.3714615130 \\
\hline & $b 0$ & 0.0000000894 & 0.0072920013 & 0.0000019087 & 0.0004261021 & -0.0028003152 & -0.0024734394 & -0.0016085733 & -2.7552698413 \\
\hline \multirow{6}{*}{$y_{3}$} & $b 5$ & 0 & 0 & -0.0038665483 & 0 & 0 & 0 & 0 & 0 \\
\hline & $b 4$ & 0 & 0 & -0.2351553374 & 0.0000653202 & 0.3334750819 & 0.0023662572 & 0.0015624268 & 0.0039707882 \\
\hline & $b 3$ & 0 & 0 & 0.1036103206 & 0.4022769915 & -0.0748973720 & 0.2503254657 & 0.1508118546 & 0.2069935964 \\
\hline & $b 2$ & 0.7610818160 & 0.0879853685 & 0.1651986422 & 0.0707971505 & -0.1686508411 & -0.0586246364 & -0.0416713834 & -0.0806495677 \\
\hline & $b 1$ & 0.0009873382 & 0.1332497450 & 0.0141241480 & 0.0016807930 & -0.0155037590 & -0.1187766192 & -0.0791482395 & -0.0860416179 \\
\hline & bo & 0.0000586507 & -0.0192131380 & 0.0000099600 & 0.0000033189 & -0.0000063465 & -0.0088194219 & -0.0059006502 & -0.0047182138 \\
\hline \multirow{6}{*}{$y_{4}$} & $b 5$ & 0 & 0 & 0 & 0 & 0 & 0 & 0.0011738672 & 0 \\
\hline & $b 4$ & 0 & 0 & -0.2670754044 & 0 & 0 & 0 & 0.0649046836 & 0.0985489683 \\
\hline & $b 3$ & 0 & 0 & -0.0254540389 & 0 & 366.5466737365 & 0.3576309291 & -0.0045028046 & -0.0262994496 \\
\hline & $b 2$ & 0.4676111287 & 0.0567671619 & 0.1596314933 & 16.9692099343 & 128.2578911893 & -0.1924386477 & -0.0588573853 & -0.0655091469 \\
\hline & $b 1$ & 0.0305870794 & 0.1077690557 & 0.0000037001 & 3.1760631184 & -438.3503112003 & -0.1741303201 & -0.0024768821 & -0.0004834947 \\
\hline & bo & 0.0000478667 & -0.0207364407 & 0.0000486728 & 0.0075766919 & -21.9426757804 & -0.0065944953 & -0.0000523056 & -0.0001182751 \\
\hline \multirow{6}{*}{$y_{5}$} & $b 5$ & 0 & 0 & 0 & 0 & 0 & 0 & 0 & 0 \\
\hline & $b 4$ & 0 & 0 & -0.0053917706 & 0.2905631499 & 0 & 0 & 0.0367873596 & 0 \\
\hline & $b 3$ & 0 & 0 & -0.2973991307 & 0.1573012703 & 0 & 0.1276513480 & 0.0086615083 & 0 \\
\hline & $b 2$ & 0 & 0.0365243746 & -0.0374266176 & 0.0171685033 & 0.1571108624 & -0.1367863356 & -0.0471219762 & 0.7919520356 \\
\hline & $b 1$ & 0.1315696810 & 0.0880926956 & 0.2232469978 & 0.0000930574 & -0.1858893549 & -0.0059733543 & -0.0003102722 & -0.8466611454 \\
\hline & bo & 0.0005817011 & -0.0201821327 & 0.0003432590 & 0.0000097933 & -0.0045550288 & -0.0004777067 & -0.0001379901 & -0.0510918158 \\
\hline \multirow{6}{*}{$y_{6}$} & $b 5$ & 0 & 0.0000707234 & 0 & 0 & 0.0010990922 & 0.0007250703 & 0.0005175091 & 0 \\
\hline & b4 & 0 & -0.0021435431 & -0.0806746116 & 0 & 0.1361960283 & 0.1043612171 & 0.0615058756 & 0 \\
\hline & $b 3$ & 0 & 0.0006377914 & 0.0353710261 & 0 & -0.0373723520 & -0.0243133637 & -0.0170871854 & 0.0833111531 \\
\hline & $b 2$ & 0.0815953559 & 0.0289230789 & 0.0319658425 & 0 & -0.0554827045 & -0.0425548187 & -0.0277869460 & -0.0263668555 \\
\hline & $b 1$ & 0.1864655531 & 0.0573189313 & 0.0002866695 & 514.8636293363 & -0.0035866558 & -0.0031687589 & -0.0017197081 & -0.0351883223 \\
\hline & bo & 0.0115851820 & -0.0072259817 & 0.0000802179 & 365.3251647928 & -0.0000723344 & -0.0000358835 & -0.0000081742 & -0.0021230027 \\
\hline \multirow{6}{*}{$y_{7}$} & $b 5$ & 0 & 0 & 0 & 0 & 0 & 0 & 0 & 0 \\
\hline & $b 4$ & 0 & 0 & 0 & 0.0004502139 & 0.0012719269 & 0.0007935494 & 0 & 0 \\
\hline & $b 3$ & 0.0500173719 & 0 & 0 & 0.0692417993 & 0.1612725505 & 0.1055173894 & 0 & 0.4069008106 \\
\hline & $b 2$ & 0.0045825393 & 0.0132484841 & -2.7496224761 & 0.0144507119 & 0.0243388723 & 0.0185549211 & 0.3704492862 & 0.1748114023 \\
\hline & $b 1$ & 0.0000814015 & 0.0106539823 & -2.0246407717 & 0.0000311329 & -0.0154721412 & -0.0133039617 & 0.0130572166 & -0.0468989629 \\
\hline & $b 0$ & 0.0000003379 & -0.0025413087 & -0.1399467106 & 0.0000059129 & -0.0010789953 & -0.0011855497 & -0.0242764892 & -0.0033795972 \\
\hline \multirow{6}{*}{$y_{8}$} & $b 5$ & -0.0061329133 & 0 & 0 & 0 & 0 & 0 & 0 & 0 \\
\hline & $b 4$ & 0.0674823406 & 0 & 0 & -0.0002233522 & 0.1161332399 & 0.0722334552 & 0.0336153509 & 0.0226864396 \\
\hline & $b 3$ & 0.0909113303 & 0 & 0 & 0.0001473781 & -0.1337467241 & -0.0867857635 & -0.0423134413 & -0.0428496923 \\
\hline & $b 2$ & 0.0049449922 & 0 & 0.0029813265 & -0.0000020191 & -0.0055539412 & -0.0002096741 & -0.0002188660 & -0.0001005361 \\
\hline & $b 1$ & 0.0000110758 & 0.0194225230 & 0.0000069287 & 0.0000003528 & -0.0000686603 & -0.0001883988 & -0.0000794953 & -0.0000720798 \\
\hline & bo & 0.0000005686 & -0.0082295989 & 0.0000002450 & -0.0000000022 & -0.0000024656 & -0.0000004229 & -0.0000004637 & -0.0000003046 \\
\hline \multirow{6}{*}{$y_{9}$} & $b 5$ & 0 & 0 & -0.0000670460 & 0 & 0 & 0 & 0 & 0 \\
\hline & b4 & 0.0428659356 & -0.0011640654 & -0.0016707917 & 0 & 0 & 0 & 0 & 0 \\
\hline & $b 3$ & 0.0044701796 & 0.0029307348 & 0.0020057569 & 0 & 0.0237937628 & -0.0014162852 & 0.0087702761 & -0.0058547035 \\
\hline & $b 2$ & 0.0001204638 & 0.0004043665 & 0.0006396034 & 0.0007659521 & -0.0621915416 & -0.0163681898 & -0.0260241408 & -0.0135911429 \\
\hline & bl & 0.0000099848 & -0.0001087345 & 0.0000321955 & 0.0000411847 & -0.0053259803 & 0.0000035590 & -0.0022290695 & -0.0025697199 \\
\hline & bo & 0.0000000338 & -0.0000015105 & 0.0000000827 & 0.0000000495 & -0.0000036601 & -0.0000189281 & -0.0000009189 & -0.0001108412 \\
\hline \multirow{6}{*}{$y_{10}$} & $b 5$ & 0 & 0 & -0.0001871928 & 0 & 0 & 0 & 0 & -0.0002760305 \\
\hline & $b 4$ & 0 & 0 & -0.0008754998 & -0.0000424609 & 0 & 0 & 0 & 0.0344216368 \\
\hline & $b 3$ & 0.4770412366 & 0 & 0.0007616054 & 0.0001743711 & 0.0811476813 & 0.0472161638 & 0 & -0.0192596998 \\
\hline & $b 2$ & 0.0427129458 & 0.0047917692 & 0.0005645629 & 0.0000067874 & -0.0048425156 & -0.0022512850 & 0.0771350424 & -0.0019015794 \\
\hline & $b 1$ & 0.0010259441 & -0.0021258065 & 0.0000509866 & 0.0000004324 & -0.0232991587 & -0.0195810535 & -0.0363200351 & -0.0000716104 \\
\hline & bo & 0.0000021075 & -0.0002939552 & 0.0000000442 & 0.0000000062 & -0.0013160002 & -0.0011003716 & -0.0035566393 & -0.0000025134 \\
\hline \multirow{6}{*}{$y_{n l}$} & $b 5$ & -0.0074984476 & 0 & -0.0000399708 & 0 & 0.0006513002 & 0 & 0.0004555181 & 0 \\
\hline & b4 & 0.1196562177 & 0 & -0.0078493606 & 0 & 0.0695097696 & 0 & 0.0251706822 & 0 \\
\hline & b3 & 0.0059563206 & 0 & 0.0057130378 & -0.0021894093 & -0.0473057556 & 0.0391406608 & -0.0180913643 & -0.0142529372 \\
\hline & $b 2$ & 0.0002889589 & 0.0152611939 & 0.0009538667 & 0.0012671029 & -0.0767660486 & -0.1121803292 & -0.0300844740 & -0.0397909026 \\
\hline & $b 1$ & 0.0000007541 & 0.0005537411 & 0.0012427103 & -0.0000023441 & -0.0046033002 & -0.0067681569 & -0.0005399470 & -0.0036641346 \\
\hline & bo & 0.0000000272 & -0.0061921005 & -0.0000001013 & 0.0000001215 & -0.0000015056 & -0.0000105181 & -0.0000292672 & -0.0001114199 \\
\hline
\end{tabular}


Table 39 - Plant 3 - Sub-model 3 - Transfer Function Denominator Parameters.

\begin{tabular}{|c|c|c|c|c|c|c|c|c|c|}
\hline$P I$ & $D$ & $u_{l}$ & $u_{2}$ & $u_{3}$ & $u_{4}$ & $u_{5}$ & $u_{6}$ & $u_{7}$ & $d_{l}$ \\
\hline \multirow{6}{*}{$y_{t}$} & $a 5$ & 1 & 1 & 1 & 0 & 0 & 0 & 1 & 1 \\
\hline & a4 & 10.0743618760 & 22.3195357228 & 2.3807299891 & 1 & 1 & 1 & 1.2426368266 & 2.8478862808 \\
\hline & $a 3$ & 3.2669003890 & 37.5581722754 & 0.7558476485 & 10.9508581644 & 1.4467858531 & 1.5330178970 & 1.9584587876 & 0.8586887061 \\
\hline & $a 2$ & 0.2654940855 & 51.8079960721 & 0.0827640983 & 73.7523822460 & 0.3538643391 & 0.4174437988 & 0.7402260218 & 0.0625464979 \\
\hline & $a l$ & 0.0039795489 & 23.6105478840 & 0.0076051303 & 106.3817633764 & 0.0298714886 & 0.0315086395 & 0.0565011183 & 0.0004421940 \\
\hline & $a 0$ & 0.0000116848 & 2.0274519205 & 0.0005964805 & 10.7222616849 & 0.0007352679 & 0.0003637264 & 0.0000245216 & 0.0000314724 \\
\hline \multirow{6}{*}{$y_{2}$} & $a 5$ & 1 & 1 & 1 & 0 & 0 & 0 & 0 & 1 \\
\hline & a4 & 0.3064254033 & 3.3279063067 & 1.4293206215 & 0 & 1 & 1 & 1 & 877.0097335184 \\
\hline & $a 3$ & 0.0299090799 & 7.1557031627 & 1.6174547945 & 1 & 1.9140948352 & 2.0376476667 & 1.7796079593 & 2302.5053671870 \\
\hline & $a 2$ & 0.0016507981 & 7.1003741540 & 0.3423169820 & 2.2021592050 & 1.5451026108 & 1.6809645969 & 1.5387310274 & 2122.1197711066 \\
\hline & al & 0.0000036660 & 4.4406647366 & 0.0097797255 & 0.6000390521 & 0.2939022199 & 0.3369089355 & 0.3262248811 & 478.3482993331 \\
\hline & $a 0$ & 0.0000001985 & 0.5215519962 & 0.0000255622 & 0.0112123588 & 0.0138955259 & 0.0167325261 & 0.0167366249 & 25.5683792976 \\
\hline \multirow{6}{*}{$y_{3}$} & $a 5$ & $I$ & 1 & 1 & 0 & 1 & 0 & 0 & 0 \\
\hline & a4 & 1.2432355983 & 2.2878429309 & 2.0270504441 & 1 & 1.7883133121 & 1 & 1 & 1 \\
\hline & $a 3$ & 2.7229958523 & 4.6901035782 & 1.8833749371 & 1.9429153827 & 1.5628362967 & 1.7839304864 & 1.6949184537 & 1.9194037364 \\
\hline & $a 2$ & 0.5641778065 & 4. 1244824828 & 0.4563927422 & 0.2849638219 & 0.3881395207 & 1.5310258606 & 1.4819354788 & 1.5884350013 \\
\hline & $a l$ & 0.0003168514 & 2.5428021684 & 0.0265948288 & 0.0065140682 & 0.0225717884 & 0.3530697158 & 0.3491997474 & 0.3173077429 \\
\hline & $a 0$ & 0.0000638298 & 0.4085835373 & 0.0000193856 & 0.0000134297 & 0.0000080616 & 0.0175354047 & 0.0176110847 & 0.0127228591 \\
\hline \multirow{6}{*}{$y_{4}$} & $a 5$ & $I$ & 1 & 1 & 1 & 1 & 1 & 1 & 1 \\
\hline & $a 4$ & 1.8176546621 & 2.5246325958 & 1.7728054806 & 4.2139327200 & 2888.1017115377 & 3.5316298469 & 1.4503840695 & 1.7439677312 \\
\hline & $a 3$ & 2.4166150798 & 4.5521251244 & 1.5524816256 & 42.2623597151 & 4331.3758213331 & 5.3257134355 & 1.2346945258 & 1.2868243207 \\
\hline & $a 2$ & 0.3995741933 & 4.0895213119 & 0.1753820414 & 65.7577245736 & 4421.8239154723 & 2.8045059616 & 0.1799405931 & 0.1418375468 \\
\hline & $a l$ & 0.0162002038 & 2.0435529055 & 0.0004765443 & 7.1659258228 & 688.9334662087 & 0.3488662655 & 0.0063822833 & 0.0031768415 \\
\hline & $a 0$ & 0.0000158008 & 0.2082186948 & 0.0000536776 & 0.0171750961 & 22.9831825695 & 0.0095397551 & 0.0001130320 & 0.0002328264 \\
\hline \multirow{6}{*}{$y_{5}$} & $a 5$ & 0 & 1 & 0 & 1 & 0 & 1 & 1 & 0 \\
\hline & a4 & 0 & 2.8209609881 & 1 & 2.5379894457 & 1 & 1.9535917224 & 1.5051624825 & 1 \\
\hline & $a 3$ & 1 & 4.6684291569 & 2.9443368004 & 0.5682052084 & 1.8589399057 & 2.3570512483 & 1.1708846096 & 14.9612715849 \\
\hline & $a_{2}$ & 0.8628689758 & 4.3036295632 & 2.3317549959 & 0.0346934996 & 2.2030809228 & 0.3057810875 & 0.1096919050 & 19.6177188069 \\
\hline & $a l$ & 0.0717501875 & 1.8493013335 & 0.2143933291 & 0.0003273922 & 0.2432045354 & 0.0165932925 & 0.0040901254 & 2.7837558904 \\
\hline & $a 0$ & 0.0002858408 & 0.1493587927 & 0.0003350367 & 0.0000191659 & 0.0045366420 & 0.0006606032 & 0.0002827755 & 0.0951420273 \\
\hline \multirow{6}{*}{$y_{6}$} & $a 5$ & 0 & 1 & 1 & 0 & 1 & 1 & 1 & 0 \\
\hline & a4 & 1 & 2.6525240745 & 1.9575012423 & 0 & 1.9076335660 & 2.0002483172 & 1.8629682761 & 1 \\
\hline & $a 3$ & 1.5120611823 & 5.3008584329 & 1.7936829623 & 1 & 1.6856787667 & 1.7683788805 & 1.6734046610 & 2.1690679258 \\
\hline & $a 2$ & 2.0616541162 & 5.1728427996 & 0.3285743034 & 2346.8953679495 & 0.4101603761 & 0.4476076169 & 0.4190578610 & 1.9663779107 \\
\hline & $a l$ & 0.6069485517 & 3.2679630391 & 0.0065131391 & 9581.9363781622 & 0.0216427093 & 0.0250991244 & 0.0198776847 & 0.4731496483 \\
\hline & $a 0$ & 0.0277187089 & 0.6609924080 & 0.0007791340 & 4451.3530379269 & 0.0003959710 & 0.0002672234 & 0.0000930676 & 0.0212815222 \\
\hline \multirow{6}{*}{$y_{7}$} & $a 5$ & 0 & 1 & 0 & 0 & 0 & 0 & 0 & 1 \\
\hline & $a 4$ & 1 & 1.6550176194 & 1 & 1 & 1 & 1 & 1 & 4.6063138578 \\
\hline & $a 3$ & 0.2484307556 & 3.2348714335 & 4.3351093896 & 2.2970187596 & 1.9415969650 & 1.9529978584 & 4.6394969994 & 10.0356624239 \\
\hline & $a 2$ & 0.0157165059 & 2.3118883799 & 10.4245591662 & 0.2428666730 & 1.3642438970 & 1.4659156708 & 8.9055200257 & 8.5564226519 \\
\hline & al & 0.0002446194 & 1.2444913235 & 6.6850430803 & 0.0009448162 & 0.2179783555 & 0.2664712796 & 4.7599487810 & 1.4836220101 \\
\hline & $a 0$ & 0.0000007919 & 0.1354913470 & 0.7099741284 & 0.0000997273 & 0.0085959504 & 0.0122783426 & 0.4365046410 & 0.0567851754 \\
\hline \multirow{6}{*}{$y_{8}$} & $a 5$ & $I$ & 0 & 1 & 0 & 1 & 1 & 1 & 1 \\
\hline & $a 4$ & 0.7400256815 & 1 & 0.4380010397 & 1 & 2.9973293627 & 2.4032097350 & 1.9122620070 & 1.6508469249 \\
\hline & $a 3$ & 0.0997378266 & 2.9765392495 & 1.6446002301 & 0.0599332589 & 0.4053510814 & 0.2597754241 & 0.2124547192 & 0.1613104735 \\
\hline & $a 2$ & 0.0034288142 & 3.2691971293 & 0.1868047340 & 0.0029567707 & 0.0125427085 & 0.0052866947 & 0.0044522173 & 0.0035389580 \\
\hline & al & 0.0000113145 & 2.0357321967 & 0.0005748348 & 0.0001525765 & 0.0002070692 & 0.0005487653 & 0.0003911328 & 0.0002696587 \\
\hline & $a 0$ & 0.0000003799 & 0.2036518082 & 0.0000156348 & 0.0000000899 & 0.0000044013 & 0.0000003166 & 0.0000016728 & 0.0000012668 \\
\hline \multirow{6}{*}{$y_{9}$} & $a 5$ & 1 & 1 & 1 & 0 & 1 & 1 & 1 & 1 \\
\hline & $a 4$ & 0.1838661485 & 1.2395827145 & 1.1087673084 & 1 & 1.8338607997 & 0.9436008867 & 1.7472743539 & 1.7043842367 \\
\hline & $a 3$ & 0.0105930448 & 1.3779075374 & 1.8226076098 & 16.3617758085 & 4.6971194602 & 1.7607926589 & 4.2801811327 & 2.0247788214 \\
\hline & $a 2$ & 0.0004103052 & 0.4540738570 & 0.2506308915 & 3.1126787162 & 0.8682593271 & 0.1417280586 & 0.8012335813 & 0.5126643830 \\
\hline & $a l$ & 0.0000183994 & 0.0438305128 & 0.0089262734 & 0.1797342025 & 0.0322600152 & 0.0019764066 & 0.0296732771 & 0.0439373167 \\
\hline & $a 0$ & 0.0000000168 & 0.0004600290 & 0.0000192615 & 0.0001831791 & 0.0000277081 & 0.0001579702 & 0.0000166244 & 0.0011323786 \\
\hline \multirow{6}{*}{$y_{I 0}$} & $a 5$ & 1 & 1 & 1 & 0 & 0 & 0 & 0 & 1 \\
\hline & a4 & 10.0303256873 & 2.2527884640 & 0.7882580906 & 1 & 1 & 1 & 1 & 2.8224679454 \\
\hline & $a 3$ & 1.9397990796 & 3.0374012638 & 1.7260037312 & 0.3784133174 & 3.3957164524 & 3.0819720997 & 2.6836894774 & 0.5743383799 \\
\hline & $a 2$ & 0.1124659418 & 2.3487844871 & 0.3131519759 & 0.0049896221 & 2.1726035878 & 2.2550391929 & 6.8856017745 & 0.0361704014 \\
\hline & $a l$ & 0.0021508744 & 0.5854890757 & 0.0152863809 & 0.0009263826 & 0.2965492054 & 0.3182377942 & 1.3797697118 & 0.0013404049 \\
\hline & $a 0$ & 0.0000037509 & 0.0345366390 & 0.0000232236 & 0.0000026920 & 0.0097508915 & 0.0105023650 & 0.0599055159 & 0.0000322227 \\
\hline \multirow{6}{*}{$y_{H I}$} & $a 5$ & 1 & 1 & 1 & 1 & 1 & 1 & 1 & 1 \\
\hline & a4 & 0.1714470632 & 2.8163149122 & 2.4402934066 & 1.0261510926 & 2.6453699072 & 1.9090442353 & 2.1040198687 & 1.7556032618 \\
\hline & $a 3$ & 0.0085089835 & 4.9443255372 & 2.6590151022 & 5.0872864567 & 2.3284920247 & 3.9495940648 & 1.9640064592 & 2.0503307718 \\
\hline & $a 2$ & 0.0003015557 & 4.5024750324 & 1.7287019667 & 1.2064381705 & 0.3923757289 & 0.7626984824 & 0.2587946022 & 0.4111607399 \\
\hline & $a l$ & 0.0000008950 & 2.2964122956 & 0.2043876559 & 0.0000675194 & 0.0151765909 & 0.0295677865 & 0.0056739749 & 0.0266174254 \\
\hline & $a 0$ & 0.0000000301 & 0.3523913633 & 0.0000001234 & 0.0000160119 & 0.0000042734 & 0.0000474080 & 0.0002143944 & 0.0006288629 \\
\hline
\end{tabular}


Table 40 - Plant 4 - Sub-model 1 - Transfer Function Numerator Parameters.

\begin{tabular}{|c|c|c|c|c|c|c|c|c|c|}
\hline$P I$ & $N$ & $u_{1}$ & $u_{2}$ & $u_{3}$ & $u_{4}$ & $u_{5}$ & $u_{6}$ & $u_{7}$ & $d_{l}$ \\
\hline \multirow{6}{*}{$y_{t}$} & $b 5$ & 0 & 0 & -0.0003398582 & 0 & 0 & 0 & 0 & 0 \\
\hline & $b 4$ & 0 & 0 & -0.0128412996 & 0 & 0 & 0 & 0 & 0 \\
\hline & $b 3$ & 0 & 0.0026264473 & -0.0037183714 & 0.0006134323 & 0.2000838754 & 0.2266069046 & 0 & -0.0065871858 \\
\hline & $b 2$ & 0.0061243144 & 0.0831890611 & 0.0031908585 & 0.1497157262 & -0.0927013696 & -0.0854313423 & 0.1540238991 & 0.0000465880 \\
\hline & $b 1$ & 0.2244401151 & 0.0410823410 & -0.0000067821 & 0.0774527353 & -0.1905269933 & -0.2119712587 & -0.1016525640 & 0.0003774270 \\
\hline & bo & 0.0819371965 & -0.0172775025 & 0.0000055159 & 0.0074412121 & -0.0082899544 & -0.0140656791 & -0.1409328227 & 0.0000310042 \\
\hline \multirow{6}{*}{$y_{2}$} & $b 5$ & 0 & 0 & -0.0003021472 & 0 & 0 & 0 & 0 & 0 \\
\hline & b4 & 0 & 0 & -0.0512095361 & 0 & 0 & 0 & 0 & 0 \\
\hline & b3 & 0 & 0 & 0.2803122228 & 0.5417407438 & 0 & 0 & 2.4538636436 & -0.0942408770 \\
\hline & $b 2$ & 25.7075822962 & 0.7998142125 & 0.1452219134 & 0.4910952854 & 1.0061286592 & 1.2620750798 & -0.9043532802 & 0.0244584000 \\
\hline & $b 1$ & 55.7570703564 & 0.5160450721 & 0.3845366713 & 0.4607010355 & -1.2777362802 & -1.5163945644 & -2.6360488410 & 0.0157011996 \\
\hline & bo & 11.9648468751 & -0.1907211448 & 0.0576317456 & 0.0003823091 & -0.4580917922 & -0.5068752753 & -0.3408726849 & 0.0007352902 \\
\hline \multirow{6}{*}{$y_{3}$} & $b 5$ & 0 & 0 & 0 & 0 & 0 & 0 & 0 & 0.0127278626 \\
\hline & $b 4$ & 0 & 0 & 0 & 0 & 0 & 0 & 0 & -0.0428284385 \\
\hline & $b 3$ & 0 & 0.8034483315 & 0 & 2.7781586283 & 11.7045942151 & 0 & 1.7812271397 & 0.0025038550 \\
\hline & $b 2$ & 0.4162919516 & 0.3276532755 & 2.0151904942 & 3.5419922850 & 6.3441837096 & 6.9348203850 & -0.3094577362 & -0.0161289950 \\
\hline & $b l$ & 7.6014871675 & -0.4052022066 & 1.7057527905 & 2.8822344228 & -22.6724407442 & 1.8900124851 & -1.6807697765 & -0.0034702875 \\
\hline & bo & 1.0729820159 & -0.0479669569 & 0.5269880492 & 0.4324463116 & -0.4187732684 & -12.5499752187 & -3.6163840643 & -0.0000593525 \\
\hline \multirow{6}{*}{$y_{4}$} & $b 5$ & 0 & 0 & -0.0014093235 & -0.0000116922 & 0 & 0 & 0 & 0.0067977883 \\
\hline & $b 4$ & 0 & 0 & -0.1165209181 & 0.7575281282 & 0.0007740098 & 0 & 0 & -0.0025527132 \\
\hline & $b 3$ & 0 & 0.5351739296 & 0.3163992407 & 3.3189244621 & 0.1273603186 & 0.1280274375 & 330.7636535001 & -0.0457772012 \\
\hline & $b 2$ & 2.6421893073 & -0.1371362948 & 0.1646189750 & 4.6100959103 & 0.6845088906 & 0.8322515018 & 379.6819497745 & -0.0005222381 \\
\hline & $b l$ & 4.4165018718 & -0.1306128933 & 0.0075591955 & 1.5666867430 & -0.7769205868 & -1.1016578108 & -1029.7347565748 & -0.0196991917 \\
\hline & bo & 0.9612782594 & -0.0016481139 & 0.0001466214 & 0.1715775790 & -0.6867056662 & -0.4602403714 & -76.3813793547 & -0.0002068662 \\
\hline \multirow{6}{*}{$y_{5}$} & $b 5$ & 0 & 0 & 0 & 0 & 0 & 0 & 0 & 0.0063662791 \\
\hline & $b 4$ & 0 & -0.0016245740 & -0.1860813615 & 0 & 0.0000237138 & 0 & 0 & -0.0346477925 \\
\hline & $b 3$ & 0.0053895371 & 0.0483617033 & 0.2140502433 & 0 & 0.2389612908 & 0 & 0 & 0.0040165453 \\
\hline & $b 2$ & -0.8376299223 & 0.1526180904 & 0.1505437602 & 864.9228577558 & -0.3497851651 & 0 & -0.1792667512 & -0.0103420215 \\
\hline & $b l$ & 2.0313675005 & -0.0978635515 & 0.0023302227 & 538.6023498569 & -0.0000512058 & -0.9029434218 & -0.2581992596 & -0.0003131471 \\
\hline & bo & 0.7146459775 & -0.0032493318 & 0.0000080778 & 33.2321870313 & -0.0000042460 & -0.0124842603 & -0.0456436148 & -0.0000346203 \\
\hline \multirow{6}{*}{$y_{6}$} & $b 5$ & 0 & 0 & 0 & 0 & 0 & -0.0000378755 & 0 & 0 \\
\hline & $b 4$ & -0.0023199932 & 0 & 0.0001513809 & 0 & -0.0147384266 & 0.1525516448 & 0 & 0 \\
\hline & $b 3$ & 0.3083462785 & 0.3604294067 & 0.1280724574 & -0.0004423233 & 1.6638619913 & 1.6391517611 & 1.2095534113 & 0 \\
\hline & $b 2$ & -0.0498637629 & 0.3249959981 & 0.1188537356 & 0.5079953789 & -0.5572261664 & -0.2339821176 & -0.7101241574 & -0.0008121933 \\
\hline & $b 1$ & 7.0275456640 & -0.2098827211 & 0.1892933991 & 0.9604068347 & -1.4067286888 & -1.3697314009 & -0.8339964915 & -0.0000421838 \\
\hline & bo & 4.6456963606 & -0.0361146598 & 0.0343235710 & 1.5900767538 & -1.0237564982 & -0.8606980441 & -0.7551138863 & -0.0000011240 \\
\hline \multirow{6}{*}{$y_{7}$} & $b 5$ & -0.0090660443 & 0 & 0 & 0 & 0 & 0 & 0 & 0 \\
\hline & b4 & 0.1361154939 & 0 & 0 & 0.0001145976 & 0 & 0 & 0 & 0.0007311580 \\
\hline & $b 3$ & 0.0022618497 & 0.1332832943 & 0 & 0.2100079488 & 0 & 0.3988102577 & 0.2078353239 & 2.1279349046 \\
\hline & $b 2$ & 0.0003617123 & -0.0798180321 & -4.1525012819 & 0.4952729480 & 0.6536951406 & -0.2925474588 & -0.1455037857 & 0.2462434441 \\
\hline & $b 1$ & 0.0000061067 & -0.0029373397 & -5.4899970700 & 0.8227220641 & 0.0720564407 & -0.0527760191 & -0.0108792681 & 0.0132675641 \\
\hline & bo & 0.0000000244 & -0.0000698705 & -0.4665294065 & 0.1110047450 & -0.4805551391 & -0.0004983488 & -0.0000340141 & 0.0003645931 \\
\hline \multirow{6}{*}{$y_{8}$} & $b 5$ & 0 & 0 & 0.0000597834 & 0.0007357677 & 0 & 0 & 0 & -0.0018952202 \\
\hline & $b 4$ & 0 & 0 & 0.0379109711 & -0.0096537698 & 0 & 0 & 0 & -0.0209951969 \\
\hline & $b 3$ & 17.9085383789 & 0.0502573669 & -0.0281694100 & 0.0030610460 & 0 & 0 & 0.0550107877 & 0.0150684643 \\
\hline & $b 2$ & 14.2989020973 & -0.0378907289 & 0.0190454649 & -0.0003417507 & 2.4344135378 & 4.5893289523 & -0.1856982552 & 0.0137340047 \\
\hline & $b 1$ & 0.0413756694 & -0.0401359615 & -0.0065323388 & -0.0000026770 & -6.8225717849 & -11.9956767385 & -0.0055775454 & 0.0014356325 \\
\hline & bo & 0.0353942091 & -0.0003006556 & -0.0001084591 & -0.0000000649 & -1.1151799933 & -2.1266618392 & -0.0000327975 & 0.0000295554 \\
\hline \multirow{6}{*}{$y_{9}$} & $b 5$ & 0 & 0 & 0.0000153605 & 0 & 0 & 0 & 0 & -0.0016968762 \\
\hline & $b 4$ & 0 & 0 & 0.0433638547 & 0 & 0 & 0 & 0 & -0.0523684977 \\
\hline & $b 3$ & 0 & 0.1216246751 & -0.0315856378 & -0.0208308522 & -0.1258832777 & -0.0004202275 & -0.0155618198 & 0.0230860542 \\
\hline & $b 2$ & 0 & -0.1731245054 & 0.0228995092 & 0.0061303242 & -0.0471205992 & 1.4952302455 & -0.0085044686 & 0.0251735267 \\
\hline & $b l$ & 5.8587166577 & -0.1040261685 & -0.0107070135 & -0.0006162257 & -0.0040961430 & -2.1842165275 & -0.0007288477 & 0.0001947329 \\
\hline & bo & 1.2572294049 & -0.0042478468 & 0.0000639651 & -0.0000083170 & -0.0000162427 & -0.1852276197 & -0.0000000692 & 0.0000114825 \\
\hline \multirow{6}{*}{$y_{I o}$} & $b 5$ & 0 & 0 & 0.0001623035 & 0 & 0 & 0 & 0 & -0.0018353810 \\
\hline & $b 4$ & 0 & 0 & 0.0673089495 & -0.0000705401 & 0 & 0 & 0 & -0.0511717960 \\
\hline & $b 3$ & 0 & 0 & -0.0478286314 & 0.0364262148 & -0.0006138581 & 0 & 0 & 0.0566271846 \\
\hline & $b 2$ & 67.3228702090 & 0 & 0.0354885129 & -0.0568154112 & 4.8151792017 & 7.2711146122 & -0.1444461821 & 0.0265605461 \\
\hline & $b 1$ & 65.1898780482 & -0.3766336028 & -0.0149266589 & 0.0251602309 & -5.0707628558 & -23.3401941305 & -0.0943238049 & 0.0001725883 \\
\hline & $b 0$ & 6.1194779458 & -0.0295829676 & 0.0000269414 & -0.0002475046 & -0.2711509269 & -1.0229894540 & -0.0080360579 & 0.0000098536 \\
\hline \multirow{6}{*}{$y_{I I}$} & $b 5$ & 0 & 0 & 0.0000729156 & 0 & 0 & -0.0001046413 & 0.0000790164 & -0.0007400098 \\
\hline & $b 4$ & 0 & 0.0009572892 & 0.0335610378 & 0 & 0 & 0.0420177578 & -0.0089247789 & -0.0458348978 \\
\hline & $b 3$ & 34.7328738569 & 0.0360991818 & -0.0204032260 & -0.0123940750 & 0 & 0.0061682197 & -0.0056313028 & 0.0217620767 \\
\hline & $b 2$ & 149.7146353288 & -0.0217138523 & 0.0177874122 & 0.0036153116 & -0.1061529931 & -0.0998908739 & -0.0004151771 & 0.0061949148 \\
\hline & $b l$ & 211.9329117753 & -0.0379328131 & -0.0067663973 & -0.0004301009 & -0.0449717204 & -0.1068282487 & -0.0000038389 & 0.0059083117 \\
\hline & bo & 7.9725150141 & -0.0016385502 & 0.0000512682 & 0.0000001888 & -0.0024735329 & -0.0071522208 & -0.0000000153 & 0.0002043971 \\
\hline
\end{tabular}


Table 41 - Plant 4 - Sub-model 1 - Transfer Function Denominator Parameters.

\begin{tabular}{|c|c|c|c|c|c|c|c|c|c|}
\hline$P I$ & $D$ & $u_{I}$ & $u_{2}$ & $u_{3}$ & $u_{4}$ & $u_{5}$ & $u_{6}$ & $u_{7}$ & $d_{I}$ \\
\hline \multirow{6}{*}{$y_{l}$} & $a 5$ & 0 & 1 & 1 & 0 & 1 & 1 & 0 & 1 \\
\hline & a4 & 1 & 1.4310356657 & 1.5300342720 & 0 & 7.5664111867 & 6.7517748643 & 1 & 1.1968113695 \\
\hline & $a 3$ & 1.5051111115 & 7.5700842960 & 1.0963742323 & 1 & 12.7208676101 & 12.0716958067 & 7.5024400032 & 3.3962490304 \\
\hline & $a 2$ & 7.4169785709 & 5.8684641201 & 0.1409186049 & 2.9016356768 & 44.1303902649 & 39.0577980873 & 12.1557370115 & 1.9926054656 \\
\hline & $a I$ & 7.7931002049 & 4.1895987456 & 0.0018661321 & 1.1071529227 & 8.0526663279 & 8.1744919072 & 43.7226058855 & 0.1817518562 \\
\hline & $a 0$ & 0.7434371304 & 0.6012698262 & 0.0002357837 & 0.0906439202 & 0.2635752321 & 0.3622728472 & 5.6493230391 & 0.0072951879 \\
\hline \multirow{6}{*}{$y_{2}$} & a5 & 1 & 0 & 1 & 1 & 0 & 0 & 1 & 1 \\
\hline & $a 4$ & 24.9192674822 & 1 & 1.4556148667 & 2.0640118257 & 1 & 1 & 9.6736345825 & 0.9444083596 \\
\hline & $a 3$ & 39.0330191398 & 4.5616318581 & 7.4292535253 & 8.5341879776 & 2.9848768579 & 2.7533134574 & 16.8866948666 & 4.6431892700 \\
\hline & $a 2$ & 170.4561979683 & 6.0458689893 & 5.7854794915 & 6.4780560205 & 8.7681958480 & 8.5748965244 & 61.6221913792 & 2.6698502243 \\
\hline & $a 1$ & 135.1313777736 & 4.4245277880 & 4.3381063811 & 3.7353562555 & 16.4440816855 & 14.8825350401 & 24.9285950267 & 0.2794188537 \\
\hline & $a 0$ & 11.6522745734 & 1.3548231433 & 0.4679873303 & 0.0030049472 & 1.9224797160 & 1.6806460932 & 1.8111710258 & 0.0089944397 \\
\hline \multirow{6}{*}{$y_{3}$} & $\overline{a 5}$ & 0 & 1 & 1 & 1 & 1 & 0 & 1 & 1 \\
\hline & a4 & 1 & 2.2235090102 & 1.5893511178 & 2.9477449893 & 17.3650717970 & 1 & 3.9764398975 & 0.6696306549 \\
\hline & $a 3$ & 1.9198606205 & 4.1445082185 & 9.8664684038 & 9.9602199460 & 29.8171970838 & 8.5546248222 & 13.5568590279 & 1.3957643639 \\
\hline & $a 2$ & 7.6364848442 & 2.5444676306 & 8.3635074670 & 8.9028131896 & 112.4565817953 & 16.8347496654 & 26.5294376475 & 0.4030660559 \\
\hline & al & 9.6414558468 & 1.2321880609 & 4.7155359059 & 5.1896611052 & 61.0371653104 & 52.6562397855 & 30.2499309752 & 0.1150427621 \\
\hline & $a 0$ & 1.0392233352 & 0.1216400926 & 0.8153013200 & 0.6679085620 & 1.0658437748 & 26.5303460717 & 11.4829845836 & 0.0019393849 \\
\hline \multirow{6}{*}{$y_{4}$} & a5 & 1 & 1 & 1 & 1 & 0 & 0 & 1 & 1 \\
\hline & a4 & 2.0451027987 & 2.3869909926 & 1.5595698955 & 3.0931794755 & 1 & 1 & 1540.5467235021 & 1.0504385254 \\
\hline & $a 3$ & 8.4148592758 & 2.0656313608 & 1.0638060472 & 8.2370461169 & 1.7717729840 & 1.3771657499 & 1692.8621395940 & 1.7257225360 \\
\hline & $a 2$ & 11.5553478853 & 1.1132392951 & 0.1914516807 & 6.5540534108 & 7.3734374784 & 7.0621753652 & 9842.6140330749 & 0.7519770527 \\
\hline & al & 6.7932215235 & 0.1933147877 & 0.0078128713 & 1.8282749101 & 7.1975155934 & 4.8837683753 & 3173.3688805233 & 0.2381913576 \\
\hline & $a 0$ & 0.7569482897 & 0.0023103117 & 0.0001390057 & 0.1616522463 & 1.2763815793 & 0.7068522318 & 173.6876366201 & 0.0023958705 \\
\hline \multirow{6}{*}{$y_{5}$} & $a 5$ & 0 & 0 & 1 & 0 & 0 & 1 & 1 & 1 \\
\hline & $a 4$ & 1 & 1 & 1.6536260237 & 1 & 1 & 1.5200038855 & 1.0248247799 & 0.6105907366 \\
\hline & $a 3$ & 1.2717583562 & 0.8247667431 & 0.9099498559 & 561.6705535950 & 3.4260892405 & 6.6818758076 & 5.7851659695 & 0.4929077323 \\
\hline & $a 2$ & 7.6643313092 & 0.6116011208 & 0.1103990847 & 2116.3690068822 & 0.4909558345 & 7.8799568053 & 4.0837687143 & 0.0887919200 \\
\hline & $a l$ & 4.5480104264 & 0.1007742127 & 0.0015616005 & 465.2998285062 & 0.0000610418 & 1.1516158362 & 1.0467712581 & 0.0040572789 \\
\hline & $a 0$ & 0.4417052223 & 0.0025655637 & 0.0000052608 & 21.5096342472 & 0.0000087122 & 0.0141489770 & 0.0778441036 & 0.0002478887 \\
\hline \multirow{6}{*}{$y_{6}$} & as & 0 & 1 & 0 & 0 & 1 & 1 & 1 & 1 \\
\hline & $a 4$ & 1 & 2.4722993181 & 1 & 0 & 5.5310082166 & 4.2915726868 & 5.1174385867 & 0.5455877402 \\
\hline & $a 3$ & 4.8422964324 & 5.2838349997 & 2.5773991590 & 1 & 13.6855997346 & 11.7726089219 & 12.7690686626 & 0.4060343822 \\
\hline & $a 2$ & 10.7849311103 & 3.8287974090 & 2.2889499243 & 3.2412553170 & 35.0939815038 & 25.8040893288 & 32.0646416746 & 0.1127666741 \\
\hline & $a 1$ & 30.0232912374 & 2.0058289341 & 1.5437144273 & 7.3288534454 & 23.0308253221 & 17.2165748405 & 19.4851729110 & 0.0155963146 \\
\hline & $a 0$ & 13.0157871611 & 0.3185325008 & 0.2054692391 & 6.0368993544 & 8.0518188585 & 5.5549924748 & 7.3843111007 & 0.0003694925 \\
\hline \multirow{6}{*}{$y_{7}$} & as & 1 & 1 & 0 & 0 & 0 & 1 & 1 & 0 \\
\hline & a4 & 0.6876230199 & 1.6756014279 & 1 & 1 & 1 & 1.2351969108 & 0.8781266014 & 1 \\
\hline & $a 3$ & 0.0144901228 & 1.5191109203 & 7.5004446872 & 2.5382487046 & 4.4741645112 & 7.6073840964 & 7.2235453449 & 52.5914111217 \\
\hline & $a 2$ & 0.0016588350 & 0.6240684587 & 20.1860222632 & 6.5936670137 & 10.4438953587 & 4.9782043358 & 3.0632089524 & 15.5564218341 \\
\hline & $a 1$ & 0.0000284225 & 0.0198395409 & 22.2301944851 & 5.1310146112 & 24.6285626282 & 0.5104835662 & 0.1636455799 & 0.6417332982 \\
\hline & $a 0$ & 0.0000001178 & 0.0004464652 & 3.3288044173 & 0.5144525226 & 5.5131859016 & 0.0046414989 & 0.0005307659 & 0.0324638587 \\
\hline \multirow{6}{*}{$y_{8}$} & $a 5$ & 0 & 0 & 1 & 1 & 1 & 1 & 1 & 1 \\
\hline & a4 & 1 & 1 & 2.2834285098 & 0.7361934238 & 23.2917737232 & 32.9978236388 & 0.5227568859 & 1.0198573960 \\
\hline & $a 3$ & 128.1096262889 & 0.8751229881 & 3.3655508539 & 0.6682385593 & 28.0097801648 & 35.8459226914 & 7.4915580779 & 1.6201772496 \\
\hline & $a 2$ & 13.5265784174 & 0.5984408253 & 1.9815801894 & 0.1293683913 & 180.2463157736 & 248.0431429582 & 1.2235727340 & 0.2400528614 \\
\hline & al & 0.3135662275 & 0.1269642665 & 0.7018978009 & 0.0018210495 & 62.9416337527 & 95.4629104453 & 0.0316386306 & 0.0146339974 \\
\hline & $a 0$ & 0.0331020382 & 0.0009331525 & 0.0556494005 & 0.0000137470 & 4.4360274053 & 6.9432224419 & 0.0001739910 & 0.0002517855 \\
\hline \multirow{6}{*}{$y_{9}$} & a5 & 0 & 1 & 1 & 1 & $I$ & 0 & 0 & 1 \\
\hline & $a 4$ & 1 & 2.8774817779 & 1.9975628151 & 1.8196117710 & 4.9313265380 & 0 & 1 & 1.2301641696 \\
\hline & $a 3$ & 3.5201045868 & 3.7468674675 & 4.3108201672 & 3.2190756211 & 3.1124754464 & 1 & 0.6121829692 & 2.6663573679 \\
\hline & $a 2$ & 11.3523377538 & 2.1082986490 & 2.1957117712 & 1.7278428156 & 0.7998668610 & 38.2894970079 & 0.1961658126 & 0.1833966924 \\
\hline & al & 25.0589286693 & 0.6859999002 & 1.0858653720 & 0.8101479707 & 0.0429804975 & 29.8038380497 & 0.0099224608 & 0.0025313115 \\
\hline & $a 0$ & 1.4576923813 & 0.0433312827 & 0.0680591883 & 0.0635245170 & 0.0001539894 & 1.4326232980 & 0 & 0.0000768671 \\
\hline \multirow{6}{*}{$y_{10}$} & a5 & 1 & 1 & 1 & 0 & 0 & 1 & 1 & 1 \\
\hline & a4 & 41.2042469847 & 2.4807062576 & 2.1980315679 & 1 & 0 & 45.8230933608 & 0.8453182590 & 1.1850543188 \\
\hline & $a 3$ & 63.8444072346 & 4.5163979514 & 4.1805006285 & 1.9839329158 & 1 & 46.1892874631 & 7.1370768490 & 2.1443668352 \\
\hline & $a 2$ & 315.6983962985 & 3.2473817181 & 2.4893141183 & 5.3268249579 & 78.5738755642 & 337.7444740749 & 4.3791286011 & 0.2301741438 \\
\hline & $a l$ & 130.6710120510 & 1.5053378891 & 1.1305768600 & 2.2893753643 & 54.6137403074 & 186.6457409890 & 1.4793886529 & 0.0021791498 \\
\hline & $a 0$ & 8.0034594481 & 0.2062010642 & 0.1290836933 & 1.6365915579 & 2.3555441659 & 6.8531784035 & 0.0946428037 & 0.0000843941 \\
\hline \multirow{6}{*}{$y_{H}$} & a5 & 1 & 0 & 1 & 1 & 1 & 1 & 1 & 1 \\
\hline & a4 & 260.8351402455 & 1 & 2.5764525272 & 1.8237906488 & 0.9793158125 & 1.4720493588 & 0.6900284371 & 2.2385459695 \\
\hline & $a 3$ & 393.1079848084 & 1.3496743573 & 4.7770743975 & 3.2916129883 & 7.1836393602 & 7.7188944322 & 0.2690712825 & 2.8832684043 \\
\hline & $a 2$ & 1842.8222284170 & 1.1241107640 & 3.3773118116 & 1.9257851593 & 4.9083162804 & 6.3283392895 & 0.0253552301 & 1.2886171481 \\
\hline & $a l$ & 1219.8069608713 & 0.4305083699 & 1.4473429964 & 0.9518143485 & 1.6308112659 & 2.9145371304 & 0.0002421833 & 0.2808015387 \\
\hline & $a 0$ & 56.5483776673 & 0.0704681441 & 0.2634815830 & 0.1329023561 & 0.1174621380 & 0.2686299870 & 0.0000011356 & 0.0112495218 \\
\hline
\end{tabular}


Table 42 - Plant 4 - Sub-model 2 - Transfer Function Numerator Parameters.

\begin{tabular}{|c|c|c|c|c|c|c|c|c|c|}
\hline$P I$ & $N$ & $u_{l}$ & $u_{2}$ & $u_{3}$ & $u_{4}$ & $u_{5}$ & $u_{6}$ & $u_{7}$ & $d_{I}$ \\
\hline \multirow{6}{*}{$y_{I}$} & $b 5$ & 0 & 0 & 0 & 0 & 0 & 0 & 0.000020134 & 0 \\
\hline & $b 4$ & 0 & 0 & 0 & 0 & 0.000398466 & 0 & -0.000253251 & 0 \\
\hline & $b 3$ & 0 & 0 & -0.016552550 & 0 & 0.028233280 & 0.038338952 & 0.021505330 & 0 \\
\hline & $b 2$ & 0 & 0.017324224 & 0.001243825 & -0.286556622 & -0.021709531 & -0.019850048 & -0.024328851 & 0.005571739 \\
\hline & $b l$ & 0.187354964 & -0.000615082 & 0.000001331 & -0.279151750 & -0.006362923 & -0.005460434 & -0.007417424 & -0.009454404 \\
\hline & bo & 0.117539195 & -0.000033825 & 0.000000067 & -0.026286594 & -0.016748395 & -0.023513438 & -0.012496691 & -0.000058000 \\
\hline \multirow{6}{*}{$y_{2}$} & $b 5$ & 0 & 0 & -0.000992203 & 0 & 0 & 0 & 0 & 0 \\
\hline & $b 4$ & 0 & 0 & -0.044847668 & -0.000650215 & 0 & 0 & 0 & 0 \\
\hline & $b 3$ & 0 & 0 & 0.230055145 & -0.087834093 & 3.365738095 & 3.840124179 & 0 & 0 \\
\hline & $b 2$ & -2.721084069 & 0.865450417 & 0.128942070 & -0.280526177 & -0.075041139 & 0.232888468 & 5.280777808 & 1.106749294 \\
\hline & $b 1$ & 28.228443970 & 0.734234202 & 0.225632437 & -0.355028683 & -3.037570876 & -3.414341381 & -5.918539823 & -2.439108668 \\
\hline & bo & 9.380630893 & -0.087626109 & 0.031206352 & -0.137135776 & -0.531898708 & -0.568287580 & -2.975087155 & -0.942868497 \\
\hline \multirow{6}{*}{$y_{3}$} & $b 5$ & -0.000288070 & 0 & -0.000708641 & 0 & 0 & 0 & 0 & 0 \\
\hline & $b 4$ & -8.404214968 & 0 & 0.344635989 & -0.632772900 & 0 & 0 & 0 & 0 \\
\hline & $b 3$ & -2.201790633 & 0 & 0.201747030 & -1.591418165 & 0 & 8.997036494 & 0 & 0 \\
\hline & $b 2$ & -18.619039587 & 2.493145331 & 0.163903268 & -2.905762632 & 6.636939676 & 3.886581081 & 5.467507154 & 8.799422737 \\
\hline & $b l$ & 42.666357426 & 0.653827569 & 0.025912555 & -1.229162998 & 0.611751935 & -13.351718221 & -2.946356692 & -7.588377190 \\
\hline & bo & 4.988559096 & -0.742737930 & 0.000010826 & -0.163368405 & -8.712412784 & -0.672290136 & -10.620238264 & -11.247295234 \\
\hline \multirow{6}{*}{$y_{4}$} & $b 5$ & 0 & 0 & 0 & 0 & 0 & 0 & 0 & 0 \\
\hline & $b 4$ & 0 & -0.005075221 & 0 & 0 & 0 & 0 & 0.158245263 & 0 \\
\hline & $b 3$ & 0 & 0.135902136 & 0.342530255 & -2.286229542 & 2.374566997 & 0 & 0.433503171 & 38.802114210 \\
\hline & $b 2$ & -3.640525174 & 0.378274122 & 0.172379711 & -1.003089457 & 2.817821496 & 1.200868568 & -0.727727973 & 34.565391161 \\
\hline & $b 1$ & 11.778151454 & -0.210310766 & 0.002206556 & -0.000377990 & -6.851112672 & -0.888637215 & -0.046948568 & -81.852410727 \\
\hline & bo & 6.012223086 & -0.003649170 & 0.000024255 & -0.000016897 & -0.182734989 & -0.883163630 & 0.000000373 & -4.184882375 \\
\hline \multirow{6}{*}{$y_{5}$} & $b 5$ & 0 & 0 & 0 & 0 & -0.013220103 & 0 & -0.000124625 & 0 \\
\hline & $b 4$ & 0 & 0 & 0 & -0.017288467 & -0.052489913 & 0 & 0.322091884 & 0.063592690 \\
\hline & $b 3$ & -1.481769722 & 0.080811039 & 0 & -1.807610924 & 0.517819136 & 0 & -0.172292828 & 0.451915138 \\
\hline & $b 2$ & 3.952607982 & 0.252226840 & -3.594827720 & -1.010538566 & -0.336570009 & 3.160829218 & -0.015876245 & -0.225946188 \\
\hline & $b l$ & 2.264843382 & -0.158864805 & 8.187266069 & -0.003043626 & -0.270145255 & -3.735341903 & -0.228937292 & -0.364031011 \\
\hline & bo & 0.040528344 & -0.000030858 & 1.727094588 & -0.001242047 & -0.080725222 & -0.015407947 & -0.035522311 & -0.019985269 \\
\hline \multirow{6}{*}{$y_{6}$} & $b 5$ & 0.000036940 & 0 & -0.001449310 & 0 & 0 & 0.000484671 & 0 & 0 \\
\hline & $b 4$ & -2.604864330 & 0 & -0.062398768 & 0 & 0 & -0.033523762 & 0 & 0 \\
\hline & $b 3$ & -0.303295663 & -0.094776080 & 0.256759991 & -759.659696318 & 2.001165157 & 2.399680445 & 1.415256942 & 0 \\
\hline & $b 2$ & -4.834292297 & 1.196258859 & 0.164628817 & -1923.836572512 & -0.030360071 & -0.283507337 & -0.246597522 & 2.837830699 \\
\hline & $b l$ & 13.504113372 & 0.348560145 & 0.385646965 & -4170.655896022 & -1.152253231 & -1.123757395 & -0.730834621 & -2.890640310 \\
\hline & bo & 1.050338099 & -0.294299778 & 0.055726739 & -3233.361033751 & -1.306927760 & -1.343330355 & -1.151170219 & -3.346119749 \\
\hline \multirow{6}{*}{$y_{7}$} & $b 5$ & 0 & 0 & 0 & 0.000067535 & 0 & 0 & 0 & 0 \\
\hline & $b 4$ & -0.007896849 & 0 & 0 & -0.157239196 & 0 & 0 & 0 & 0 \\
\hline & $b 3$ & -0.013725628 & 0 & -0.003744419 & -0.392445952 & 1.811977317 & 1.376070441 & 19.587758301 & 150.059910434 \\
\hline & $b 2$ & -1.882524837 & 0.123266508 & -1.045284110 & -0.565424589 & 1.918348392 & 1.331058074 & 23.116935986 & 110.209136021 \\
\hline & $b l$ & 4.333998709 & -0.055880291 & -0.320476857 & -0.074653627 & -1.817935398 & -1.427606894 & -18.388019412 & -91.844473398 \\
\hline & bo & 1.493673276 & -0.001530425 & -0.013988412 & -0.002501885 & -0.176023772 & -0.152423594 & -1.557455026 & -7.369667139 \\
\hline \multirow{6}{*}{$y_{8}$} & $b 5$ & 0 & 0 & -0.000428915 & -0.000028577 & 0 & 0 & 0 & 0 \\
\hline & $b 4$ & 0.003913878 & 0.045940768 & 0.025060668 & 0.008665487 & 0 & 0.004493123 & 0 & 0.043332970 \\
\hline & $b 3$ & 0.851827750 & -0.014504984 & -0.012304510 & -0.031069765 & 0 & 0.059106312 & 0.000348493 & 0.020428109 \\
\hline & $b 2$ & 0.398792899 & -0.041282418 & 0.007126912 & 0.047387631 & 0 & 0.065580814 & 2.054366923 & -0.121658832 \\
\hline & $b l$ & 0.000895513 & -0.002459604 & -0.003049851 & -0.016290545 & -1.342656433 & -0.167984245 & -0.182365016 & -0.018365256 \\
\hline & bo & 0.000285409 & -0.000082596 & -0.000165065 & 0.003336871 & -0.156209515 & -0.023576287 & -0.413165641 & -0.000152034 \\
\hline \multirow{6}{*}{$y_{9}$} & $b 5$ & 0 & 0 & -0.006859859 & 0.001638864 & 0 & 0 & 0 & 0 \\
\hline & $b 4$ & 0 & 0.002676969 & 0.003973951 & -0.040680559 & 0 & 0 & 0 & 0 \\
\hline & $b 3$ & 0.051253706 & 0.138678474 & 0.054254234 & 0.042974061 & 326.991042408 & 0 & 141.049515193 & 0 \\
\hline & $b 2$ & 2.738075235 & -0.079582091 & -0.030788232 & -0.018242320 & -56.664784351 & -0.387838936 & -42.870578290 & -0.671622415 \\
\hline & $b 1$ & 0.683826683 & -0.061156419 & -0.002638117 & 0.003895792 & -499.485142786 & -0.029742435 & -189.526441720 & -0.488930380 \\
\hline & bo & 0.000796265 & -0.002280053 & 0.000007710 & 0.000039884 & -27.770727946 & -0.000511909 & -8.776884638 & -0.034666712 \\
\hline \multirow{6}{*}{$y_{10}$} & $b 5$ & 0 & 0 & -0.006732359 & 0.000910821 & 0 & 0 & 0 & 0 \\
\hline & $b 4$ & 0 & 0 & 0.018792610 & -0.039032741 & 0 & 0 & 0 & 0 \\
\hline & b3 & 0 & 0.255528325 & 0.026313518 & 0.039624143 & 177.506870197 & 264.668137945 & 164.708034361 & 0 \\
\hline & $b 2$ & 1.942284664 & -0.156057225 & -0.015596000 & -0.015924758 & 15.918746076 & 43.259711996 & 20.932624930 & 0 \\
\hline & $b l$ & 1.381763247 & -0.209529624 & -0.002359208 & 0.002966423 & -268.569945534 & -446.903410912 & -229.275929849 & 0.122426515 \\
\hline & bo & 0.131628415 & -0.014868771 & -0.000028744 & 0.000121203 & -5.775022774 & -12.201842980 & -14.778815772 & -0.307239978 \\
\hline \multirow{6}{*}{$y_{I I}$} & $b 5$ & 0 & 0 & -0.002964140 & 0.001180279 & 0 & 0 & 0 & 0.000900129 \\
\hline & $b 4$ & 0 & 0 & 0.020153004 & -0.020010414 & 0 & 0 & 0.204262018 & 0.153827858 \\
\hline & $b 3$ & 0 & 0 & 0.002735912 & 0.020599348 & 0 & 0 & 0.262111201 & 0.172951116 \\
\hline & $b 2$ & 1.154000686 & 0.150186624 & -0.002623776 & -0.008345722 & 0.243644898 & 0.293257062 & 0.223318123 & -0.026204126 \\
\hline & $b 1$ & 0.723818592 & -0.284093499 & -0.001834165 & 0.001527119 & -0.519365631 & -0.621770998 & -0.435800972 & -0.419744912 \\
\hline & bo & 0.046646092 & -0.014998994 & -0.000003053 & 0.000032351 & -0.034543414 & -0.045025430 & -0.076908403 & -0.067006412 \\
\hline
\end{tabular}


Table 43 - Plant 4 - Sub-model 2 - Transfer Function Denominator Parameters.

\begin{tabular}{|c|c|c|c|c|c|c|c|c|c|}
\hline$P I$ & $D$ & $u_{l}$ & $u_{2}$ & $u_{3}$ & $u_{4}$ & $u_{5}$ & $u_{6}$ & $u_{7}$ & $d_{l}$ \\
\hline \multirow{6}{*}{$y_{l}$} & $a 5$ & 0 & 0 & 0 & 0 & 1 & 1 & 1 & 0 \\
\hline & $a 4$ & 1 & 1 & 1 & 1 & 2.155622771 & 2.292045623 & 2.194396518 & 1 \\
\hline & $a 3$ & 3.112705714 & 1.211797550 & 1.166153784 & 3.216797448 & 7.033246742 & 7.355976303 & 6.915465744 & 0.234923141 \\
\hline & $a 2$ & 5.513634274 & 0.910077825 & 0.229252356 & 9.015220505 & 9.602672884 & 10.324520267 & 9.741888632 & 4.890341142 \\
\hline & $a l$ & 14.083221322 & 0.153418179 & 0.000026048 & 5.119065197 & 9.007117007 & 10.342623082 & 8.387834685 & 0.684647662 \\
\hline & $a 0$ & 1.475016439 & 0.003949474 & 0.000002862 & 0.414118977 & 1.052011364 & 1.212912491 & 0.973417887 & 0.004016623 \\
\hline \multirow{6}{*}{$y_{2}$} & $a 5$ & 1 & 1 & 1 & 0 & 1 & 1 & 1 & 1 \\
\hline & a4 & 2.003156025 & 1.945718104 & 1.104274519 & 1 & 13.741847373 & 12.557777680 & 4.985178161 & 1.672233538 \\
\hline & $a 3$ & 24.662804449 & 6.667920737 & 5.406778048 & 1.230573675 & 15.366329137 & 14.320925790 & 37.897862673 & 15.437903561 \\
\hline & $a 2$ & 15.783969597 & 8.545869734 & 4.192370891 & 5.083401860 & 64.869613555 & 59.059294283 & 34.759068582 & 10.584322806 \\
\hline & al & 93.309507046 & 5.441361100 & 2.669133657 & 4.072815283 & 34.152310665 & 30.682401815 & 149.491873992 & 48.705481256 \\
\hline & $a 0$ & 10.767272048 & 1.695931378 & 0.276905656 & 1.225848685 & 3.124382278 & 2.708199824 & 22.047387802 & 6.322521099 \\
\hline \multirow{6}{*}{$y_{3}$} & $a 5$ & 1 & 1 & 1 & $I$ & 0 & 1 & 1 & 1 \\
\hline & $a 4$ & 4.955870468 & 1.986572197 & 1.068593216 & 1.616379951 & 1 & 10.920796114 & 2.806612229 & 2.679222531 \\
\hline & $a 3$ & 13.336046546 & 6.196011709 & 0.689438394 & 5.423780968 & 9.607579631 & 13.143976953 & 21.094812254 & 23.204028129 \\
\hline & $a_{2}$ & 24.609944494 & 7.817324311 & 0.301645075 & 5.041534199 & 11.057458044 & 50.842015624 & 21.862232836 & 21.915211090 \\
\hline & al & 34.757560236 & 4.154535691 & 0.034333286 & 1.855368213 & 44.168669867 & 26.923231545 & 74.783447623 & 85.062021864 \\
\hline & $a 0$ & 3.054959883 & 1.583872961 & 0.000014361 & 0.216239693 & 18.722049159 & 1.190655137 & 27.905461667 & 27.748094370 \\
\hline \multirow{6}{*}{$y_{4}$} & $a 5$ & 0 & 0 & 1 & 0 & 1 & 0 & I & 1 \\
\hline & a4 & 1 & 1 & 1.166908627 & 1 & 8.020087686 & 1 & 0.536539666 & 109.396988728 \\
\hline & $a 3$ & 3.391412434 & 1.173679795 & 0.767850052 & 2.774069274 & 8.558611223 & 1.318882274 & 4.6998888356 & 63.531957760 \\
\hline & $a 2$ & 6.394509612 & 0.883613792 & 0.126241304 & 0.690722852 & 36.400510456 & 5.174995389 & 1.486276610 & 512.287623715 \\
\hline & al & 15.294699004 & 0.233412280 & 0.001571749 & 0.000181582 & 9.863035616 & 5.240532329 & 0.072743633 & 149.846918994 \\
\hline & $a 0$ & 2.555749677 & 0.003636940 & 0.000016934 & 0.000016456 & 0.235461334 & 0.934549234 & 0.000001080 & 6.097566993 \\
\hline \multirow{6}{*}{$y_{5}$} & $a 5$ & 1 & 0 & 0 & 0 & 1 & 1 & 1 & 1 \\
\hline & $a 4$ & 1.301016207 & 1 & 1 & 1 & 0.724354657 & 3.798394618 & 2.496986046 & 0.826629819 \\
\hline & $a 3$ & 5.296408464 & 0.692935628 & 18.866376620 & 2.315255356 & 4.905712923 & 6.123238315 & 3.070811636 & 4.825295716 \\
\hline & $a 2$ & 5.140829659 & 0.477194755 & 23.335580512 & 0.387508363 & 2.481300331 & 17.182339615 & 2.068160772 & 2.960376220 \\
\hline & al & 0.696868021 & 0.065991634 & 8.022723762 & 0.003152861 & 0.740635696 & 2.653335174 & 0.511714037 & 0.497405241 \\
\hline & $a 0$ & 0.010817876 & 0.000010386 & 0.668105426 & 0.000472305 & 0.067064380 & 0.010194783 & 0.036444460 & 0.018980043 \\
\hline \multirow{6}{*}{$y_{6}$} & $a 5$ & 1 & 1 & 1 & 1 & 1 & 1 & 1 & 1 \\
\hline & a4 & 4.202775359 & 2.503292548 & 1.131162455 & 2716.306866191 & 6.980724350 & 6.608011208 & 6.290525186 & 2.443932869 \\
\hline & $a 3$ & 12.523267258 & 6.687401559 & 5.534033024 & 6350.688468002 & 12.134868173 & 10.948043960 & 11.955749212 & 21.202119164 \\
\hline & $a 2$ & 20.700137867 & 10.892444935 & 4.413139037 & 16938.621416734 & 34.765151769 & 32.399661240 & 31.800453182 & 20.674047777 \\
\hline & al & 31.905728429 & 5.229271418 & 3.117830420 & 24306.474970682 & 27.151362591 & 21.999757377 & 27.123803031 & 76.236225975 \\
\hline & $a 0$ & 2.132306688 & 2.563354644 & 0.345760873 & 12286.834591147 & 10.139655632 & 8.617958846 & 10.973854991 & 29.534373292 \\
\hline \multirow{6}{*}{$y_{7}$} & $a 5$ & 0 & 0 & 0 & 1 & 1 & 1 & 1 & 1 \\
\hline & $a 4$ & 1 & 1 & 0 & 1.525212775 & 15.646184152 & 9.598950584 & 210.905985574 & 1087.225533277 \\
\hline & $a 3$ & 5.303443402 & 1.238099881 & 1 & 4.925099060 & 13.809641105 & 10.396536529 & 124.225111871 & 607.830038956 \\
\hline & $a 2$ & 7.135499723 & 1.154373051 & 3.461369891 & 2.983126397 & 72.686084063 & 44.158168840 & 992.182692469 & 5184.142638539 \\
\hline & al & 24.692130052 & 0.337458356 & 1.640723174 & 0.327587308 & 25.190845122 & 16.220695882 & 317.118821845 & 1534.185374406 \\
\hline & $a 0$ & 3.725851691 & 0.007332189 & 0.137333984 & 0.009971212 & 1.538606432 & 1.066943676 & 17.481477999 & 81.668982358 \\
\hline \multirow{6}{*}{$y_{8}$} & $a 5$ & 0 & 1 & 1 & 1 & 1 & 0 & 0 & 1 \\
\hline & $a 4$ & 1 & 1.037804735 & 1.150751366 & 1.078926319 & 10.620238728 & 1 & 0 & 0.580672059 \\
\hline & $a 3$ & 3.441571832 & 0.688836837 & 2.643104137 & 5.393704063 & 9.298893802 & 0.621872764 & 1 & 5.082205607 \\
\hline & $a 2$ & 0.369885444 & 0.186403497 & 0.986804334 & 4.144072173 & 50.716211192 & 4.944957786 & 13.245044440 & 1.650342701 \\
\hline & al & 0.002404366 & 0.010100098 & 0.366145103 & 2.898745582 & 15.255611538 & 1.672380787 & 26.620598537 & 0.124406748 \\
\hline & $a 0$ & 0.000257916 & 0.000296204 & 0.019747089 & 0.555018068 & 0.954170552 & 0.115265617 & 3.290234465 & 0.000974310 \\
\hline \multirow{6}{*}{$y_{9}$} & $a 5$ & 0 & 0 & 1 & $I$ & 1 & 1 & 1 & 1 \\
\hline & $a 4$ & 0 & 1 & 0.574841581 & 0.874926421 & 2081.858566417 & 0.893071051 & 1154.852101751 & 3.099113873 \\
\hline & $a 3$ & 1 & 1.332626820 & 4.669464364 & 3.811312319 & 1533.809723062 & 5.129771122 & 783.027362888 & 6.169419589 \\
\hline & $a 2$ & 5.546797964 & 0.803273968 & 1.911749873 & 1.201724913 & 10397.960302253 & 2.820770453 & 5769.870189961 & 14.097644959 \\
\hline & al & 0.464500696 & 0.266577209 & 0.247826520 & 0.806155964 & 4553.865240577 & 0.175659851 & 2228.040392591 & 4.651629793 \\
\hline & $a 0$ & 0.000337547 & 0.018576602 & 0.008903303 & 0.038780140 & 194.568471961 & 0.002756182 & 82.374301497 & 0.228184832 \\
\hline \multirow{6}{*}{$y_{10}$} & $a 5$ & 0 & 1 & 1 & 1 & 1 & 1 & 1 & 0 \\
\hline & $a 4$ & 1 & 2.940893902 & 0.471556527 & 1.019301986 & 1196.164677118 & 1454.086360598 & 1440.221265216 & 0 \\
\hline & $a 3$ & 0.941133137 & 4.296061125 & 4.318490735 & 3.660794680 & 891.279795141 & 1139.879068232 & 1079.918394636 & 1 \\
\hline & $a 2$ & 5.310158870 & 2.582248497 & 1.351856713 & 1.271797525 & 5979.952511618 & 7247.742733599 & 7296.571249305 & 0.698463212 \\
\hline & $a 1$ & 2.041619389 & 0.971022250 & 0.269092432 & 0.873529212 & 2586.734072764 & 3399.874212893 & 3258.174704069 & 5.540629292 \\
\hline & $a 0$ & 0.135227419 & 0.107055050 & 0.012041953 & 0.052488754 & 50.888398675 & 83.761975500 & 179.726397389 & 2.866195599 \\
\hline \multirow{6}{*}{$y_{H}$} & $a 5$ & 0 & 1 & 1 & 1 & 0 & 0 & 1 & 1 \\
\hline & $a 4$ & 1 & 1.820863098 & 0.537477653 & 0.775483477 & 1 & 1 & 6.035273542 & 4.817942895 \\
\hline & $a 3$ & 1.025735249 & 5.724347585 & 4.016679105 & 4.026136496 & 2.792897121 & 2.609759253 & 9.202477395 & 8.121960570 \\
\hline & $a 2$ & 5.422368081 & 4.470465139 & 1.142169181 & 1.234131858 & 5.968829790 & 5.911468106 & 28.146375803 & 22.605706207 \\
\hline & al & 2.338294367 & 2.273860728 & 0.372022602 & 1.103640421 & 12.498103080 & 11.563514825 & 13.615677999 & 10.457931488 \\
\hline & $a 0$ & 0.159404936 & 0.413831050 & 0.030938263 & 0.076727245 & 0.984338095 & 1.005931680 & 2.864070074 & 1.941511649 \\
\hline
\end{tabular}


Table 44 - Plant 4 - Sub-model 3 - Transfer Function Numerator Parameters.

\begin{tabular}{|c|c|c|c|c|c|c|c|c|c|}
\hline$P I$ & $N$ & $u_{1}$ & $u_{2}$ & $u_{3}$ & $u_{4}$ & $u_{5}$ & $u_{6}$ & $u_{7}$ & $d_{I}$ \\
\hline \multirow{6}{*}{$y_{l}$} & $b 5$ & 0 & 0 & 0.0003078410 & 0 & 0 & 0 & 0 & 0 \\
\hline & $b 4$ & 0 & 0 & -0.0027553646 & 0 & 0 & 0 & 0 & 0 \\
\hline & $b 3$ & -7.8813901300 & 0.0078812927 & -0.0001749214 & 0 & 0.0596137512 & 0.0723026009 & 0.0606960333 & 0 \\
\hline & $b 2$ & 4.9541328914 & 0.0000624732 & -0.0000086551 & 0.0218501740 & -0.1062614552 & -0.1204673414 & -0.0809391419 & 0.0598309539 \\
\hline & $b l$ & 12.3178130244 & 0.0000231634 & -0.0000005514 & -0.0002644975 & -0.0554224031 & -0.0322675066 & -0.2136456849 & -0.2964347494 \\
\hline & bo & 2.7433177944 & 0.0000001944 & -04 & 0.0000548358 & -0.0043030076 & -0.0023148852 & -0.0245464575 & -0.0530336587 \\
\hline \multirow{6}{*}{$y_{2}$} & $b 5$ & 0 & 0 & 0.0025735342 & 0 & 0 & 0 & -0.0001739439 & 0.0001820523 \\
\hline & $b 4$ & 0 & 0 & 0.0006428653 & 0 & 0 & 0 & 0.0161939753 & 0.0652812839 \\
\hline & $b 3$ & -3.7259103435 & 0.2982538677 & -0.0000044088 & 0 & 0.1452709981 & 0.3039379449 & -0.0042641417 & 0.0959530254 \\
\hline & $b 2$ & 6.4027315976 & 0.2661949832 & 0.0000064794 & 0 & -0.4146535925 & -0.3886443174 & -0.3247159901 & -0.3406375149 \\
\hline & $b l$ & 14.7385191563 & 0.0036803698 & -0.0000000901 & -0.0032970027 & -0.7453754043 & -0.9007970270 & -0.5660436256 & -0.6020060776 \\
\hline & $b 0$ & 2.9664130199 & 0.0001614490 & 0.0000000039 & 0.5969388827 & -0.1812132559 & -0.2226720378 & -0.1273590702 & -0.1374126410 \\
\hline \multirow{6}{*}{$y_{3}$} & $b 5$ & 0 & 0 & 0 & 0.2148379355 & 0 & 0 & -0.0004022472 & 0 \\
\hline & $b 4$ & 0 & 0 & -0.0002274839 & 0.0455407838 & 0 & 0 & 0.1397452217 & 0 \\
\hline & $b 3$ & -7.2753955570 & 0 & 0.0567334501 & 0.0010508947 & 0.7260791905 & 1.3044547285 & -0.0708552625 & 0 \\
\hline & $b 2$ & 22.5162003030 & 0.0415266836 & 0.0002489750 & 0.0002443561 & -1.7792689222 & -1.8991308802 & -0.6947026796 & 0.8062354068 \\
\hline & bl & 24.7371142009 & 1.4667212491 & 0.0000831687 & 0.0000028039 & -3.2460030814 & -3.5168314433 & -2.5361131097 & -2.6345320116 \\
\hline & bo & 3.1066433413 & 0.0934791171 & 0.0000004437 & 0.0000002505 & -0.4826128679 & -0.5534360120 & -0.3852472905 & -2.1009419485 \\
\hline \multirow{6}{*}{$y_{4}$} & $b 5$ & 0 & 0 & 0 & 0 & 0 & 0 & 0 & 0 \\
\hline & $b 4$ & 0 & 0.0578205476 & 0 & 0 & 0 & 0 & 0 & 0 \\
\hline & $b 3$ & -3.5374611605 & 0.3958542231 & 0 & 1.6057105067 & 1.0371295427 & 1.4716173222 & 0.6303762003 & 2.6292978387 \\
\hline & $b 2$ & 18.3850803360 & 0.0062010162 & 0.0779134627 & 0.2578369604 & -2.0558604152 & -2.0905761374 & -2.1607916820 & -2.9160987990 \\
\hline & $b l$ & 15.5197100247 & 0.0034799171 & -0.0001658711 & 0.0001858585 & -3.9886381413 & -3.9116724679 & -4.0820321347 & -8.0222565668 \\
\hline & bo & 3.7189101007 & 0.0000221356 & 0.0000204785 & 0.0005729095 & -0.4358631948 & -0.3866020962 & -0.4915071372 & -0.9101977831 \\
\hline \multirow{6}{*}{$y_{5}$} & $b 5$ & 0 & 0.0168763667 & 0 & 0 & 0 & 0 & -0.0002019120 & 0 \\
\hline & $b 4$ & 0 & 0.7138329887 & 0 & 0.6297708807 & 0.0043678842 & 0.0049621034 & 0.2414631185 & 0.0127874532 \\
\hline & $b 3$ & -2.3912330893 & 0.0896270025 & 0.0918977124 & 0.0016066214 & 0.1188198121 & 0.1576053729 & 0.1010573980 & -0.1001860790 \\
\hline & $b 2$ & 19.1918862637 & 0.6424556650 & 0.0011669429 & 0.0119001517 & 0.2279822084 & 0.5063255249 & -0.6900756684 & 0.8830177020 \\
\hline & bl & 12.8274346528 & -0.0017544871 & 0.0000523001 & -0.0001396960 & -1.4187740071 & -1.7790433460 & -2.6598370573 & -3.1916821448 \\
\hline & $b 0$ & 3.9573114461 & 0.0009800918 & 0.0000005973 & 0.0000213813 & -0.0086490951 & 0.0388360880 & -0.0951124650 & -0.0492664494 \\
\hline \multirow{6}{*}{$y_{6}$} & $b 5$ & 0 & 0 & 0 & 0.0706733612 & 0 & 0 & 0 & 0 \\
\hline & $b 4$ & 0 & 0 & 0 & 0.0207584516 & 0 & 0 & 0 & 0 \\
\hline & $b 3$ & 0 & 1.1422873934 & 0 & 0.0003050577 & 0 & 0 & 0 & 0 \\
\hline & $b 2$ & -3.6793826383 & 0.2089576785 & -0.0000017571 & 0.0001239599 & 0.5884360208 & 2.9370625725 & -0.1395774466 & 0.2533465661 \\
\hline & $b 1$ & 9.5841412199 & 0.1015553508 & 0.0000000923 & 0.0000007729 & -2.7643235579 & -7.1870654159 & -1.7390703202 & -2.6686161234 \\
\hline & $b 0$ & 12.8287734424 & 0.0002450712 & -0.0000000059 & 0.0000001551 & -2.9266581400 & -1.5511477030 & -1.2772386764 & -1.1216775777 \\
\hline \multirow{6}{*}{$y_{7}$} & $b 5$ & 0 & 0 & 0 & 0 & 0 & 0 & 0 & 0 \\
\hline & $b 4$ & 0 & 0 & 0.0013207961 & 0 & 0 & 0 & 0 & 0 \\
\hline & $b 3$ & -4.7369986868 & 1.5226125518 & -5.4907178795 & 0.4342480658 & 1.5165193172 & 1.8952107035 & 1.1115668572 & 1.6515426592 \\
\hline & $b 2$ & 27.2943342986 & 0.3971392293 & 0.3539705973 & 0.0050559156 & -1.6550793034 & -1.5428689075 & -1.6544466951 & -1.8591646953 \\
\hline & $b 1$ & 19.8943984548 & -1.0060839887 & 0.0037242815 & 0.0003102355 & 0.5430445077 & 0.3695229902 & 0.7206037151 & 0.1295402550 \\
\hline & bo & 4.1049546164 & -0.0195030845 & 0.0005297982 & 0.0000013332 & -2.9919447908 & -4.0657177262 & -2.1374718336 & -2.7249294431 \\
\hline \multirow{6}{*}{$y_{8}$} & $b 5$ & 0 & 0 & 0 & -0.0049913910 & 0 & 0 & 0 & 0 \\
\hline & $b 4$ & -0.2700879067 & 0 & 0.0004720191 & 0.0003199634 & 0.0400323534 & 0 & 0.0342744355 & 0 \\
\hline & $b 3$ & 0.9082320035 & 0 & -0.0001422605 & -0.0000297072 & 0.0477446649 & 0 & 0.0204463981 & 0 \\
\hline & $b 2$ & 0.3162546063 & 0.1193010041 & -0.0000592973 & -0.0000021678 & -0.0181810912 & 187.2644745804 & -0.0063905770 & 0.2616489506 \\
\hline & $b l$ & 0.0021617371 & -0.1360649405 & -0.0000002589 & 0.0000000069 & -0.0839258531 & -248.8398577541 & -0.0527751717 & -0.2986450039 \\
\hline & bo & 0.0002243505 & -0.0053275442 & -0.0000000134 & -0.0000000037 & -0.0180215969 & -48.7561413701 & -0.0158245099 & -0.0421932408 \\
\hline \multirow{6}{*}{$y_{9}$} & $b 5$ & 0 & 0 & 0 & 0.0046545298 & 0 & 0 & 0 & 0 \\
\hline & $b 4$ & 0 & 0 & 0.0080896454 & -0.0092309452 & 0 & 0 & 0 & 0 \\
\hline & $b 3$ & -0.0796596767 & 0 & 0.0293368128 & 0.0014494796 & -0.1097502565 & 0 & 28.0651836004 & 0 \\
\hline & $b 2$ & -0.4691883298 & 4.0346612533 & -0.0100402948 & -0.0000972361 & -0.0013398234 & 0 & -15.6285868917 & -0.6161125285 \\
\hline & $b 1$ & 3.1559813145 & -4.3477479498 & -0.0009828369 & 0.0000099379 & -0.0000171260 & -0.1523553064 & -0.9176168419 & -0.4609611627 \\
\hline & bo & 0.4521325467 & -0.1872372600 & 0.0000000784 & -0.0000003611 & -0.0000001877 & -0.0071834667 & -0.0000681906 & -0.0238351651 \\
\hline \multirow{6}{*}{$y_{10}$} & $b 5$ & 0 & -0.0021910236 & 0 & 0.0022362523 & 0 & 0 & 0 & 0 \\
\hline & $b 4$ & 0 & -0.3223348117 & -0.0004705139 & -0.0103374293 & 0 & 0 & 0 & 0 \\
\hline & $b 3$ & 0 & 0.8015688802 & -1.4997430261 & 0.0007161044 & 0.4278241411 & 0 & 0 & 0 \\
\hline & $b 2$ & 14.0470914044 & -0.1805404152 & -0.3886000768 & -0.0000591954 & -0.5096854962 & 0.6933844728 & -0.2132064054 & 0.8640926443 \\
\hline & $b 1$ & 17.2729521233 & -0.6816666187 & -1.3476226948 & 0.0000008382 & -0.1182214281 & -0.4783611514 & -0.0850120317 & -1.2478573533 \\
\hline & bo & 2.4406479625 & -0.0508723970 & -0.0371398115 & -0.0000003245 & -0.0490667508 & -0.7888206341 & -0.0036959423 & -2.6133627232 \\
\hline \multirow{6}{*}{$y_{11}$} & $b 5$ & 0 & 0 & -0.0015501843 & 0.0046944234 & 0 & 0 & 0.0001082916 & 0 \\
\hline & $b 4$ & 0 & 0 & 0.0001870485 & -0.0086720126 & 0 & 0 & 0.1553330975 & 0 \\
\hline & $b 3$ & 0.2197181967 & 0.2921456187 & 0.0147135768 & 0.0013896454 & 193.3966314454 & 1.9846473991 & 0.3012837925 & 0.5720421718 \\
\hline & $b 2$ & 7.9222512861 & 0.0975208248 & -0.0058357207 & -0.0000953928 & 97.5252492772 & 0.7142287131 & 0.1755985547 & -0.3680282601 \\
\hline & $b 1$ & 7.9048070491 & -0.4004037227 & -0.0002973473 & 0.0000083817 & -284.2048200838 & -3.1999825886 & -0.4413844782 & -0.9312461437 \\
\hline & $b 0$ & 0.5098634519 & -0.0191079371 & -0.0000017146 & -0.0000002380 & -44.7447313267 & -0.4709133226 & -0.0719020770 & -0.0542867495 \\
\hline
\end{tabular}


Table 45 - Plant 4 - Sub-model 3 - Transfer Function Denominator Parameters.

\begin{tabular}{|c|c|c|c|c|c|c|c|c|c|}
\hline$P 1$ & $D$ & $u_{I}$ & $u_{2}$ & $u_{3}$ & $u_{4}$ & $u_{5}$ & $u_{6}$ & $u_{7}$ & $d_{l}$ \\
\hline \multirow{6}{*}{$y_{I}$} & $a 5$ & 1 & 0 & 1 & 0 & 1 & 1 & 1 & 1 \\
\hline & a4 & 37.668665745 & 0 & 0.563383387 & 0 & 4.455178287 & 3.688049610 & 7.749396274 & 2.336725830 \\
\hline & $a 3$ & 134.204557841 & 1 & 0.024366163 & 1 & 8.534806442 & 7.556484148 & 15.693451990 & 16.871751274 \\
\hline & $a 2$ & 279.702759619 & 0.022939333 & 0.002100357 & 0.455190671 & 22.952452702 & 18.387657446 & 43.320117501 & 16.212893712 \\
\hline & $a l$ & 643.913018172 & 0.002268946 & 0.000068558 & 0.002362961 & 8.004184520 & 3.887523362 & 39.876372645 & 58.451079053 \\
\hline & $a 0$ & 73.419836351 & 0.000006760 & 0.000000426 & 0.001075598 & 0.506847132 & 0.227912390 & 3.509234987 & 7.010083536 \\
\hline \multirow{6}{*}{$y_{2}$} & $a 5$ & 1 & 0 & 1 & 0 & 1 & 1 & 1 & 1 \\
\hline & a4 & 4.286621273 & 1 & 0.200976094 & 0 & 2.215338018 & 2.553324469 & 1.652427756 & 1.773432579 \\
\hline & $a 3$ & 18.248501259 & 1.084668650 & 0.003532616 & 0 & 9.284081522 & 9.557856027 & 8.607355844 & 8.926285541 \\
\hline & $a 2$ & 28.300127310 & 1.188123275 & 0.000687536 & 0 & 11.680736930 & 13.590339667 & 8.373988678 & 9.142441481 \\
\hline & $a l$ & 62.103420186 & 0.016096996 & 0.000001408 & 1 & 16.663449663 & 17.336737175 & 14.481107520 & 15.439404610 \\
\hline & $a 0$ & 6.703565218 & 0.000717179 & 0.000000214 & 7.089618027 & 2.136584910 & 2.166548703 & 1.846136570 & 1.815114772 \\
\hline \multirow{6}{*}{$y_{3}$} & $a 5$ & 1 & 0 & 0 & 1 & 1 & 1 & 1 & 0 \\
\hline & $a 4$ & 3.402838222 & 0 & 1 & 0.062568677 & 3.283402112 & 3.482171523 & 2.047856039 & 1 \\
\hline & $a 3$ & 16.184455468 & 0 & 0.625646622 & 0.007389174 & 10.407865492 & 10.335989214 & 9.433209394 & 2.759999778 \\
\hline & $a 2$ & 22.200459702 & 1 & 0.001821821 & 0.000307773 & 18.038936011 & 19.113096212 & 11.029109315 & 9.972044635 \\
\hline & al & 52.416909394 & 2.343336600 & 0.000875953 & 0.000013227 & 20.591696654 & 19.720259454 & 18.200658731 & 14.621090363 \\
\hline & $a 0$ & 5.966357252 & 0.114723121 & 0 & 0.000000357 & 3.706768374 & 3.711866802 & 3.389041116 & 18.488733643 \\
\hline \multirow{6}{*}{$y_{4}$} & a5 & 1 & 0 & 0 & 0 & 1 & 1 & 1 & 1 \\
\hline & $a 4$ & 3.112517695 & 1 & 0 & 1 & 5.137619210 & 4.729366203 & 5.658405059 & 9.008899475 \\
\hline & $a 3$ & 15.293459965 & 0.317809049 & 1 & 2.722601143 & 11.747680948 & 11.155861328 & 12.436312132 & 18.707307325 \\
\hline & $a 2$ & 20.925458136 & 0.011876291 & 0.271517952 & 0.256859718 & 28.990779645 & 26.491224660 & 32.166832963 & 54.560751607 \\
\hline & $a 1$ & 48.377320501 & 0.002776032 & 0.000217752 & 0.006737287 & 23.728583964 & 21.242393345 & 26.518527379 & 53.889790971 \\
\hline & $a 0$ & 10.322528464 & 0.000013466 & 0.000059100 & 0.000629496 & 5.941111555 & 5.257763214 & 6.697151675 & 13.310593853 \\
\hline \multirow{6}{*}{$y_{5}$} & $a 5$ & 1 & 1 & 1 & 1 & 0 & 0 & 1 & 0 \\
\hline & $a 4$ & 3.096154531 & 1.543703170 & 0.967679843 & 0.348247728 & 1 & 1 & 3.405054086 & 1 \\
\hline & a3 & 14.558056605 & 1.371783393 & 0.171439966 & 0.037056660 & 1.292766412 & 1.449631574 & 8.840812629 & 2.698078259 \\
\hline & $a 2$ & 20.691449649 & 0.360381693 & 0.002732196 & 0.005491505 & 6.272892912 & 6.387721851 & 18.117970711 & 7.264406920 \\
\hline & al & 44.320367796 & 0.001931615 & 0.000101306 & 0.000083638 & 5.552822059 & 6.430535897 & 12.175877320 & 14.290741602 \\
\hline & $a 0$ & 11.329793932 & 0.000504910 & 0.000000959 & 0.000011228 & 1.378852587 & 1.591558724 & 2.836083777 & 3.041126321 \\
\hline \multirow{6}{*}{$y_{6}$} & $a 5$ & 0 & 1 & 0 & 1 & 1 & 1 & 1 & 1 \\
\hline & $a 4$ & 1 & 2.010202963 & 1 & 0.072771252 & 4.029819640 & 5.736085753 & 2.921012209 & 2.459827501 \\
\hline & $a 3$ & 3.461511441 & 4.424298577 & 0.008840450 & 0.006873718 & 17.249503405 & 24.579073280 & 13.208387368 & 14.551588136 \\
\hline & $a 2$ & 16.144929161 & 2.676601443 & 0.002208356 & 0.000448261 & 32.961443596 & 39.911145372 & 21.304178515 & 18.654346786 \\
\hline & al & 21.479348031 & 0.695241749 & 0.000017033 & 0.000011176 & 59.784249723 & 94.028144081 & 38.257906583 & 46.145302920 \\
\hline & $a 0$ & 51.498646681 & 0 & 0.000000351 & 0.000000660 & 46.125727091 & 20.629605804 & 24.302745557 & 19.869711489 \\
\hline \multirow{6}{*}{$y_{7}$} & $a 5$ & 1 & 1 & 0 & 1 & 1 & 1 & 1 & 1 \\
\hline & $a 4$ & 3.361337463 & 1.747618431 & 1 & 13.972265975 & 3.655227730 & 3.589278046 & 3.633681923 & 3.585929911 \\
\hline & $a 3$ & 14.900058698 & 5.516573169 & 208.067182511 & 2.038111149 & 11.324550062 & 11.591648429 & 10.976552001 & 12.770029337 \\
\hline & $a 2$ & 22.028171701 & 4.037013357 & 55.504973213 & 0.031998263 & 23.478447776 & 23.526669971 & 23.015607979 & 24.234966675 \\
\hline & $a 1$ & 45.446306970 & 2.474302120 & 0.913625214 & 0.001388982 & 25.562163263 & 27.309462885 & 23.638587815 & 33.601060352 \\
\hline & $a 0$ & 9.221996153 & 0.047748225 & 0.057112469 & 0.000005479 & 19.122341631 & 20.710596164 & 17.654451564 & 21.317209892 \\
\hline \multirow{6}{*}{$y_{8}$} & $a 5$ & 1 & 0 & 0 & $I$ & 1 & 1 & 1 & 0 \\
\hline & $a 4$ & 0.591213350 & 1 & 1 & 0.030394949 & 1.334456921 & 1659.969306617 & 1.223727258 & 0 \\
\hline & a3 & 5.603612589 & 1.849337696 & 0.297193806 & 0.013766139 & 6.136414478 & 1274.231409122 & 6.069977721 & 1 \\
\hline & $a 2$ & 0.605796879 & 2.039899839 & 0.016581068 & 0.000063239 & 5.366157677 & 10222.324781857 & 4.723322908 & 16.627553321 \\
\hline & al & 0.006974788 & 0.697031648 & 0.000172939 & 0.000022751 & 1.864606163 & 4108.245551552 & 1.900667250 & 5.688014674 \\
\hline & $a 0$ & 0.000415511 & 0.023252701 & 0.000002404 & 0.000000009 & 0.164084472 & 351.206066600 & 0.188019140 & 0.408291969 \\
\hline \multirow{6}{*}{$y_{9}$} & $a 5$ & 0 & 1 & 1 & 1 & 1 & 0 & 0 & 1 \\
\hline & $a 4$ & 0 & 7.326833344 & 0.803043921 & 0.265601101 & 1.696614366 & 0 & 1 & 2.311595354 \\
\hline & $a 3$ & 1 & 18.241407367 & 4.379219787 & 0.133083650 & 0.852736611 & 1 & 184.243709888 & 6.581257273 \\
\hline & $a 2$ & 0.422443672 & 22.135597617 & 2.429657416 & 0.004765020 & 0.009474567 & 1.710834717 & 188.718615281 & 11.723631983 \\
\hline & $a 1$ & 5.584832416 & 12.844787639 & 0.300944446 & 0.001080910 & 0.000136546 & 1.002052730 & 8.979331762 & 3.978532262 \\
\hline & $a 0$ & 0.405006451 & 1.388699473 & 0.012747927 & 0.000021351 & 0.000001470 & 0.039889655 & 0.000680401 & 0.163340474 \\
\hline \multirow{6}{*}{$y_{10}$} & $a 5$ & 1 & 1 & 0 & 1 & 1 & 0 & 1 & 1 \\
\hline & $a 4$ & 3.371889803 & 2.619358216 & 1 & 0.271291792 & 1.324955297 & 1 & 0.753506290 & 1.819314737 \\
\hline & $a 3$ & 12.262307765 & 5.817827676 & 94.332872781 & 0.148615774 & 6.714073100 & 2.343479393 & 5.746548727 & 16.572745570 \\
\hline & $a 2$ & 19.797144812 & 4.735189973 & 31.497626044 & 0.005688355 & 6.261194826 & 7.464494189 & 3.473570145 & 15.999312093 \\
\hline & al & 29.816743283 & 2.118478838 & 443.842622607 & 0.001188766 & 1.620592913 & 11.937326612 & 0.951857263 & 59.570839744 \\
\hline & $a 0$ & 2.879553244 & 0.264912374 & 31.598793352 & 0 & 0.374857714 & 4.683826889 & 0.037119523 & 20.607526588 \\
\hline \multirow{6}{*}{$y_{l l}$} & $a 5$ & 1 & 1 & 1 & 1 & 1 & 1 & 1 & 1 \\
\hline & a4 & 3.156430497 & 2.322305418 & 1.000881395 & 0.509508418 & 1716.418109299 & 14.357112277 & 4.494295702 & 5.599683512 \\
\hline & $a 3$ & 11.748316358 & 5.262510809 & 4.708795418 & 0.215475737 & 1593.147203104 & 19.145725936 & 9.010398336 & 11.412691571 \\
\hline & $a 2$ & 18.215314651 & 4.683659685 & 3.569641698 & 0.012935528 & 10039.840046057 & 81.907369740 & 23.474111206 & 31.473773761 \\
\hline & $a 1$ & 27.332045731 & 2.415123661 & 0.511523727 & 0.001574812 & 5025.628356411 & 43.947829464 & 10.759617008 & 22.531573106 \\
\hline & $a 0$ & 1.976657761 & 0.415034344 & 0.015604028 & 0.000068750 & 1095.282711487 & 9.026746219 & 2.331043983 & 1.429690492 \\
\hline
\end{tabular}

